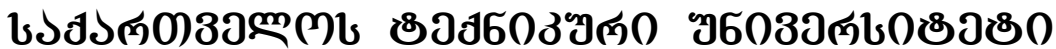

GEORGIAN TECHNICAL UNIVERSITY

\section{ГРУЗИНСКИЙ ТЕХНИЧЕСКИЙ УНИВЕРСИТЕТ}

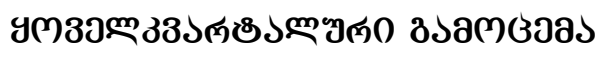

QUARTERLY PUBLICATION

ЕЖЕКВАРТАЛЬНОЕ ИЗДАНИЕ

ISSN 1512-0996

DOI:https://doi.org/10.36073/1512-0996
Certificate

$\mathrm{ICI}$ Journals master List

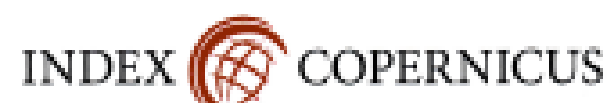

\section{ข๓ๆวปภถ WORKS ТРУДЫ}

N3(513)

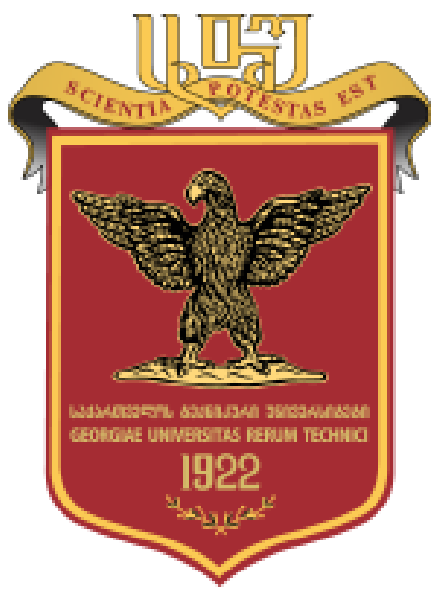

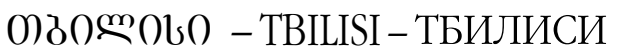


@ssбbgòyeos 1924 6gel.

зубомеэемды - 4 бмдубо бэеобьедо.

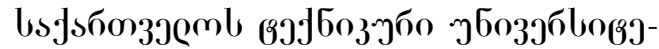

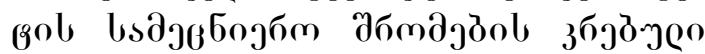

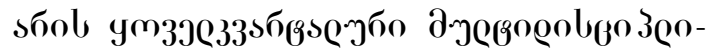

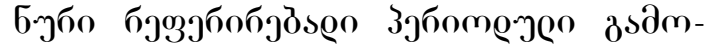

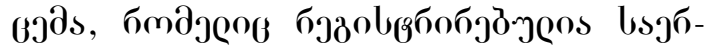

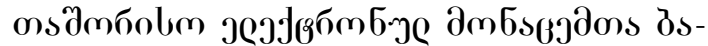
8sdo - Index Copernicus International.

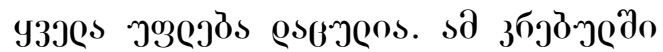

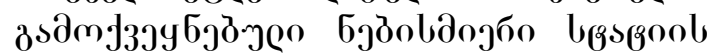
(

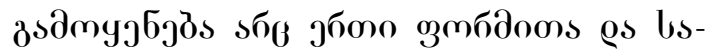

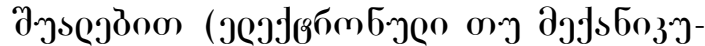

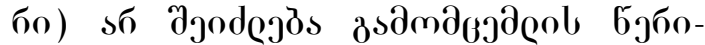

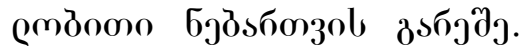

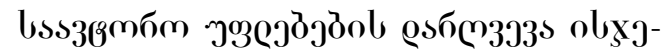
os $356 \mathrm{mboon}$.

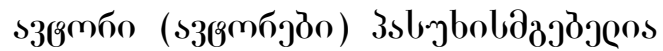

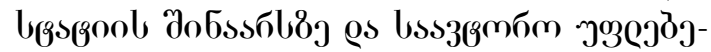

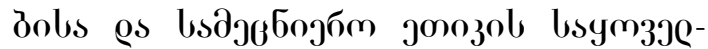

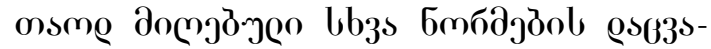
8 .

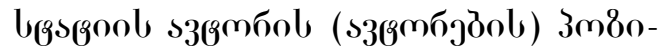

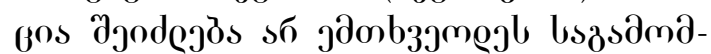
6jagm bsbeol 3m8ogosb.

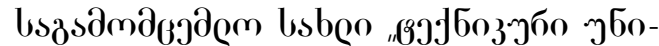

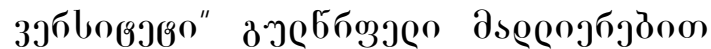

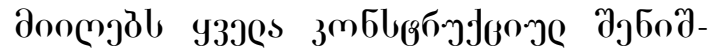

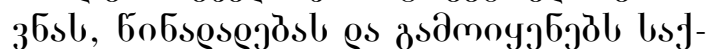

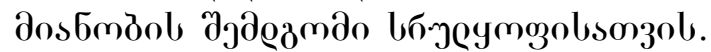

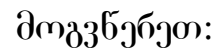

sagamomcemlosakhli@yahoo.com

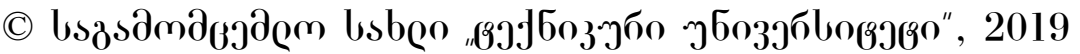

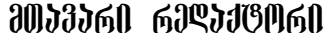

ง.

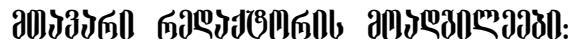

c. $3 \mathrm{eogos}_{30 \mathrm{o}}$

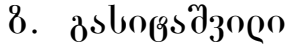

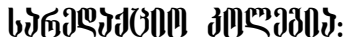

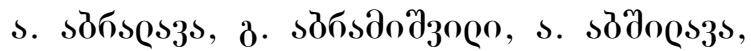

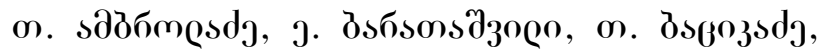

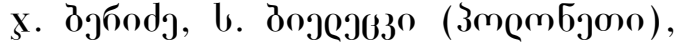

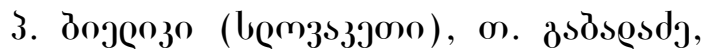

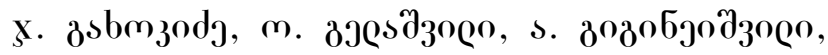

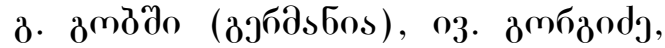

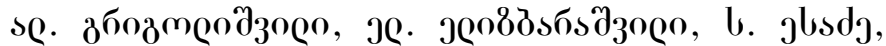

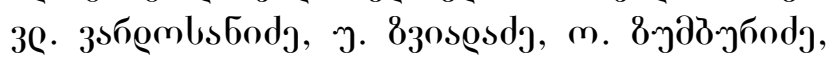

3. 8э5

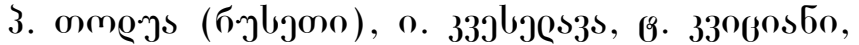

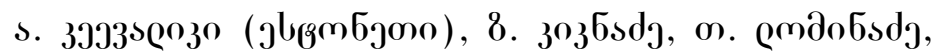

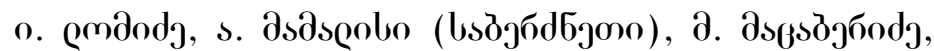

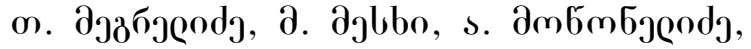

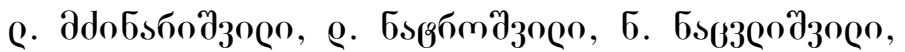

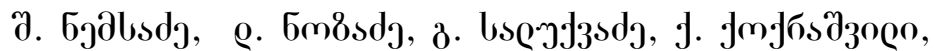

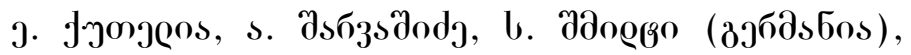

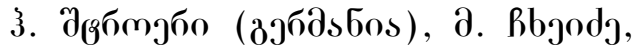

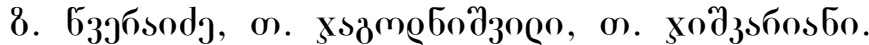

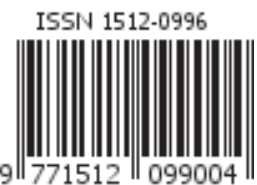


Founded in 1924.

Published in quarterly editions.

Collection of Academic Works of Georgian Technical University is a multidisciplinary quarterly refereed periodical included in Index Copernicus International.

All rights reserved. No material appearing in this publication (texts, images, illustrations and other visual) can in any form or by any means (electronic or manual) be used by other parties without prior written consent of the publisher.

Infringement of copyright is punishable by law.

Author (authors) is (are) responsible for content of the article as well as protection of copyright and compliance with generally accepted norms of academic ethics.

Judgements of the author (authors) and the publishing house may vary.

Publishing House "Technical University" is open to constructive feedback and ideas for the purpose of continuous improvement.

Contact us:

sagamomcemlosakhli@yahoo.com
Editor in Chief

A. Prangishvili

Deputy Editors in Chief

L. Klimiashvili

Z. Gasitashvili

Editorial Board:

A. Abralava, G. Abramishvili, A. Abshilava,

T. Ambroladze, E. Baratashvili, T. Batsikadze, J. Beridze,

S. Bielecki (Poland), P. Bielik (Slovakia), M. Chkheidze,

E. Elizbarashvili, S. Esadze, T. Gabadadze,

J. Gakhokidze, O. Gelashvili, A. Gigineishvili,

G. Gobsch (Germany), Iv. Gorgidze, Al. Grigolishvili,

T. Jagodnishvili, T. Jishkariani, A. Keevalik (Estonia),

Z. Kiknadze, K. Kokrashvili, E. Kutelia, I. Kveselava,

T. Kvitsiani, T. Lominadze, I. Lomidze,

A.G. Mamalis (Greece), M. Matsaberidze,

L. Mdzinarishvili, T. Megrelidze, M. Meskhi,

A. Motzonelidze, D. Natroshvili, N. Natsvlishvili,

Sh. Nemsadze, D. Nozadze, G. Salukvadze,

H. Stroher (Germany), H. Sunkel (Austria),

S.M. Schmidt (Germany), A. Sharvashidze,

D. Tavkhelidze, P. Todua (Russia), Z. Tsveraidze,

Vl. Vardosanidze, O. Zumburidze, U. Zviadadze.

(C) Publishing House "Technical University", 2019

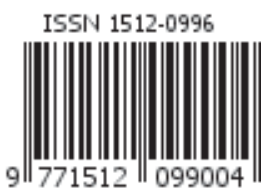


Учрежден в 1924 году.

Периодичность - 4 номера в год

Сборник научных трудов Грузинского технического университета является ежеквартальным мультидисциплинарным реферируемым периодическим изданием, которое зарегистрировано в международной базе электронных данных - Index Copernicus International.

Защищены все права. Любую опубликованную в данном сборнике статью (текст, фото, иллюстрации) невозможно использовать ни одной из форм или средствами (электронными или механическими) без письменного разрешения издателя.

Нарушение авторских прав наказуемо законом.

Автор (авторы) несет ответственность за содержание статьи и защиту всеобще принятых норм научной этики и авторских прав.

Мнение автора (авторов) статьи может не совпадать с мнением Издательского дома.

Издательский дом “Технический университет» с благодарностью учтет все конструктивные замечания, предложения и использует их для совершенствования дальнейшей деятельности.

Пишите:

sagamomcemlosakhli@yahoo.com

\section{РЕДАКЦИОННАЯ КОЛЛЕГИЯ:}

\section{ПРЕДСЕДАТЕЛЬ}

А.И. Прангишвили

\section{ЗАМ. ПРЕДСЕДАТЕЛЯ:}

Л.Д. Климиашвили

3.А. Гаситашвили

\section{ЧЛЕНЫ РЕДКОЛЛЕГИИ:}

А.Г. Абралава, Г.С. Абрамишвили, А.В. Абшилава, Т.А. Амброладзе, Е.Ш. Бараташвили, Т.В. Бацикадзе, С. Биелецки (Польша), П. Биелик (Словакия), Дж.Л. Беридзе, Вл.Г. Вардосанидзе, Т.Г. Габададзе, Дж.В. Гахокидзе, О.Г. Гелашвили,

А.В. Гигинеишвили, Г. Гобш (Германия), Ив.А. Горгидзе, Ал.Р. Григолишвили, Т.А. Джагоднишвили,

Т.С. Джишкариани, У.И. Звиададзе, О.Г. Зумбуридзе, Г. Зункел (Австрия), И.С. Квеселава, Т.А. Квициани, А. Кеевалик (Эстония), 3.Г. Кикнадзе, К.А. Кокрашвили, Е.Р. Кутелия, И.Б. Ломидзе, Т.Н. Ломинадзе, А. Мамалис (Греция),

М.И. Мацаберидзе, Л.Д. Мдзинаришвили, Т.Я. Мегрелидзе, М.А. Месхи, А.Н. Моцонелидзе, Д.Г. Натрошвили, Н.В. Нацвлишвили, Ш.А. Немсадзе, Д.А. Нозадзе, Г.Г. Салуквадзе, Д.Д. Тавхелидзе, П. Тодуа (Россия), 3.Н. Цвераидзе, М.М. Чхеидзе, А.М. Шарвашидзе,

С. Шмидт (Германия), Г. Штроер (Германия), Э.Н. Элизбарашвили, С.Ю. Эсадзе.

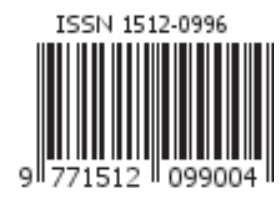




\section{มก6dimun}

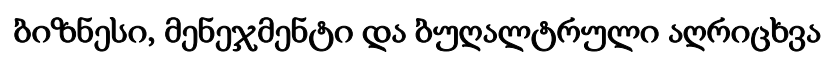

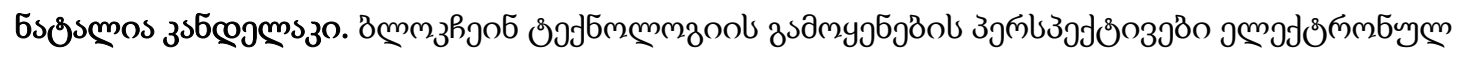

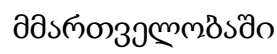
11

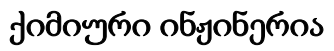

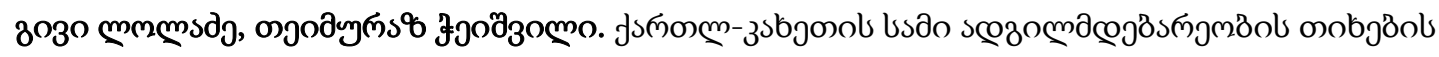

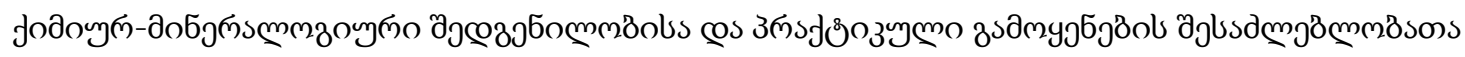

gobfs

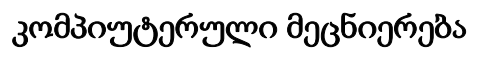

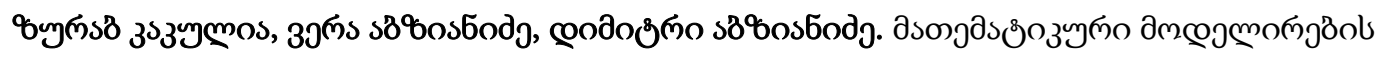

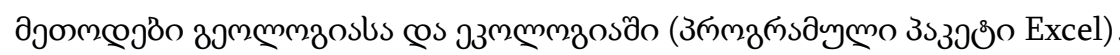

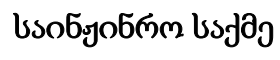

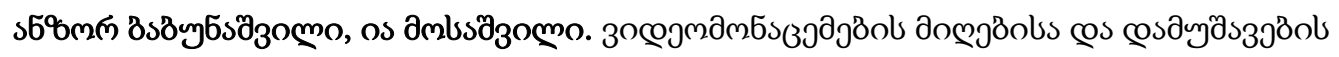

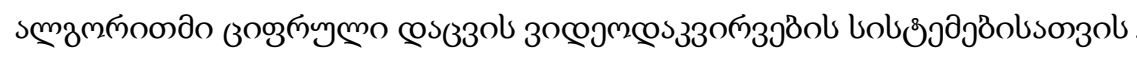

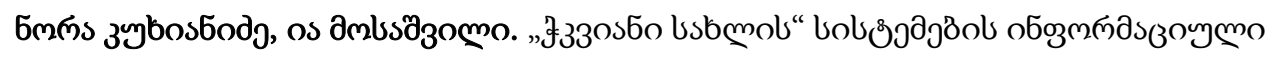

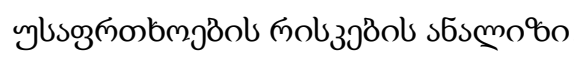

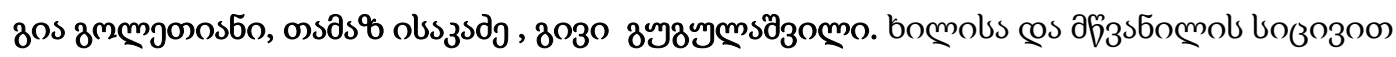

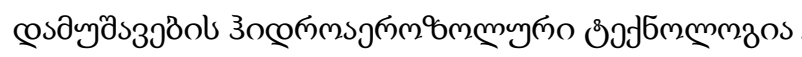

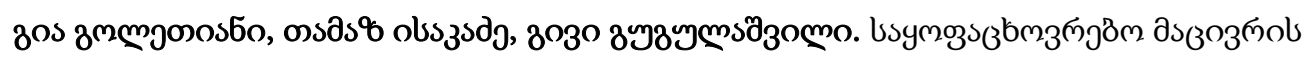

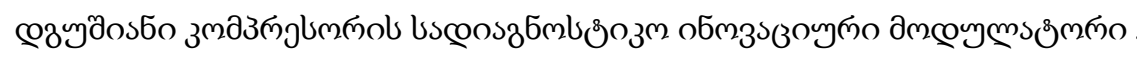

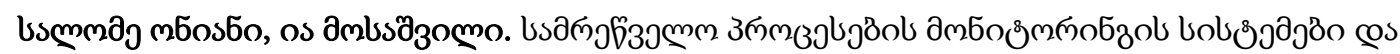

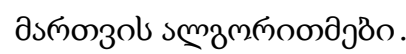

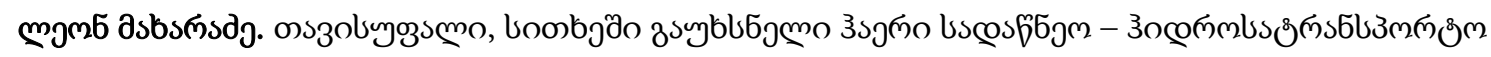

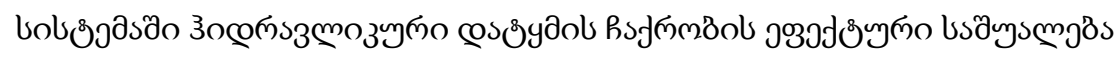
78

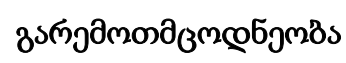

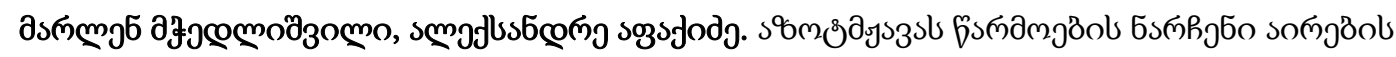

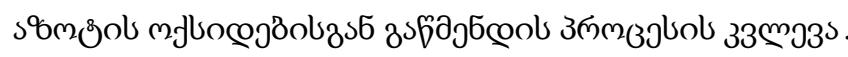
86 


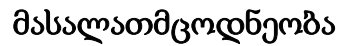

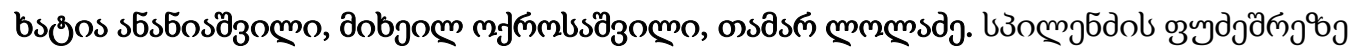

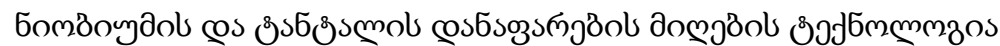

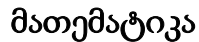

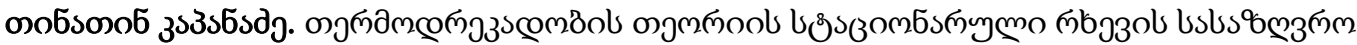

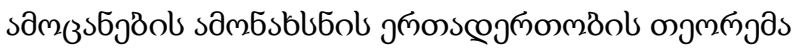

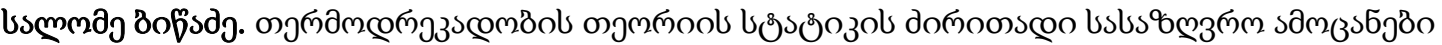

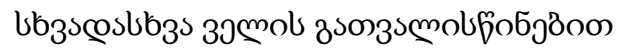

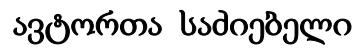

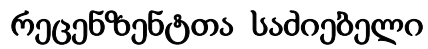

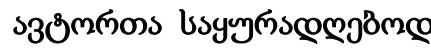




\section{CONTENTS}

\section{Business, Management and Accounting}

Natalia Kandelaki. Prospects for the use of blockchain technology in e-Government

\section{Chemical Engineering}

Givi Loladze, Teimuraz Cheishvili. Study of chemical-mineralogical composition and possibilities of practical application of clays from three locations of Kartli-Kakheti

\section{Computer Science}

Zurab Kakulia, Vera Abzianidze, Dimitri Abzianidze. Mathematical model methods in geology and ecology (Excel Software)

\section{Eengineering}

Anzor Babunashvili, Ia Mosashvili. Reception and processing algorithm of videodata for digital video surveillance system 40

Nora Kukhianidze, Ia Mosashvili. Infosecuty risk Analysis for "Smart House" systems 47

Gia Goletiani, Tamaz Isakadze, Givi Gugulashvili. Hydroelectric technology of refrigeration processing of fruits and greens 54

Gia Goletiani, Tamaz Isakadze, Givi Gugulashvili. Diagnostic innovative modulator for piston compressors of household refrigerators. 61

Salome Oniani, Ia Mosashvili. Monitoring systems of industrial processes and control algorithms 68

Leon Makharadze. Free combined air - as an effective remedy for water hammers attenuation in the pressure head hydrotransport systems

\section{Environmental Science}

Marlen Mchedlishvili, Aleksandre Aphakidze. Research of the process of exhaust gas cleaning in the production of nitric acid from nitrogen oxides. 


\section{Materiels Science}

Khatia Ananiashvili, Mikheil Okrosashvili, Tamar Loladze. Technology for obtaining of

Niobium and Tantalum coatings on the copper Substrate

\section{Mathematics}

Tinatin Kapanadze. The Uniqueness Theorem of the boundary value problems for the stationary oscilations of the Theory of Thermoelasticity...

Salome Bitsadze. Basic boundary value problems of statics of Thermoelasticity theory considering

different field

Author's index

Reviewer's index 133

Guidelines for Authors 140 


\section{СОДЕРЖАНИЕ}

Бизнес, Менеджмент и Бухгалтерский учет

Наталья Канделаки. Перспективы использования блокчейн-технологии в электронном управлении ..... 11

\section{Химическая инженерия}

Гиви Лоладзе, Теимураз Чеишвили. Изучение химико-минералогического содержания и возможности практического использования глин находящихся на трёх картлийско-кахетинских территориях.

\section{Компьютерные науки}

Зураб Какулия, Вера Абзианидзе, Дмитрий Абзианидзе. Методы математического моделирования в геологии и экологии (программный пакет Excel)

\section{Инженерное дело}

Анзор Бабунашвили, Ия Мосашвили. Алгоритм приема и обработки видеоданных для цифровых охранных систем видеонаблюдения

Нора Кухианидзе, Іа Мосашвили. Анализ рисков информационной безопасности для систем

«Умный дом».

Гия Голетиани, Тамаз Исакадзе, Гиви Гугулашвили. Гидроаэрозольная технология холодильной обработки фруктов и зелени

Гия Голетиани, Тамаз Исакадзе, Гиви Гугулашвили. Диагностический инновационный модулятор для поршневых компрессоров бытовых холодильников .

Саломе Ониани, Ия Мосашвили. Системы мониторинга производственных процессов и алгоритмы управления .

Леон Махарадзе. Свободный, нерастворенный в жидкости воздух - эффективное средство для гашения гидравлических ударов в напорных гидротранспортных системах.

Наука об окружающей среде

Марлен Мчедлишвили, Александр Апакидзе. Исследование процесса очистки отходящих газов производства азотной кислоты от оксидов азота 


\section{Материаловедение}

Хатиа Ананиашвили, Михаил Окросашвили, Тамар Лоладзе. Технология получения ниобиевых и танталовых покрытий на медной подложке

\section{Математика}

Тинатин Капанадзе. Теорема единственности решений граничных задач стационарного колебания теории термоупругости

Саломе Бицадзе. Основные граничные задачи статики теории термоупругости с учётом разных полей

Указатель авторов

Указатель рецензентов 


\section{UDC 621.385}

\section{SCOPUS CODE 1405}

DOI: https://doi.org/10.36073/1512-0996-2019-3-11-18

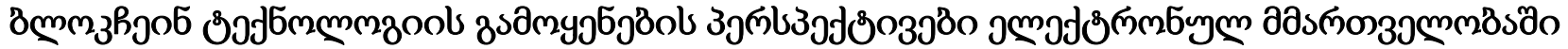

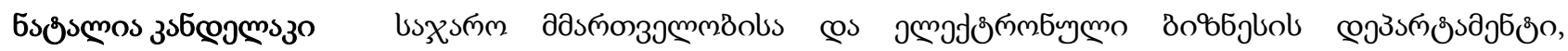

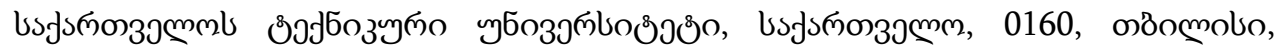
ว. зmb\&s3sl 77

E-mail: nataliakandelaki89@gmail.com

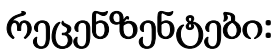

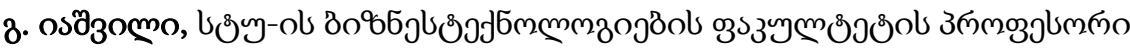

E-mail: genadi_iashvili@hotmail.com

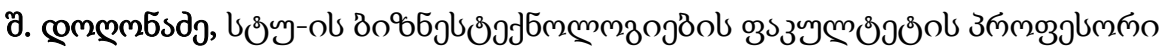

E-mail: shotad55@gmail.com

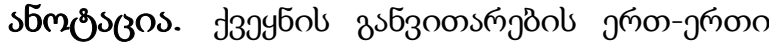

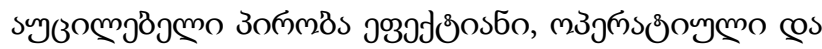

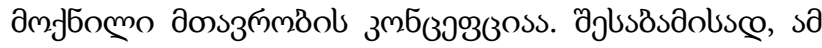

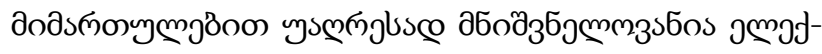

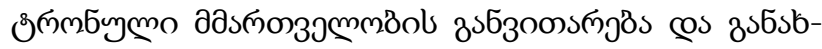

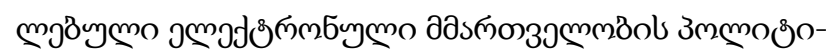

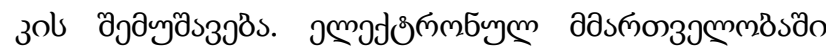

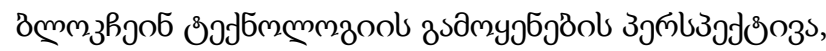

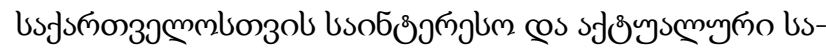

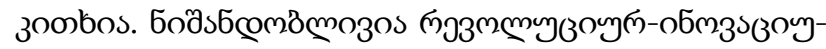

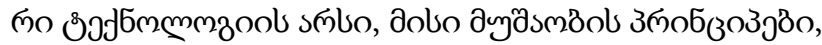

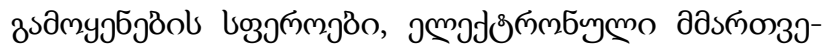

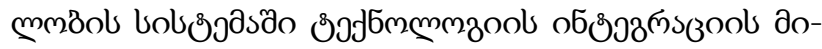

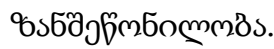

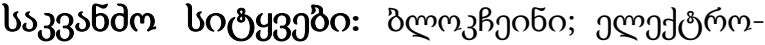

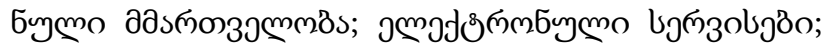

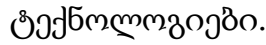

ISSN 1512-0996

\section{əjuşзsmo}

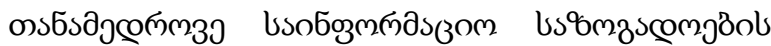

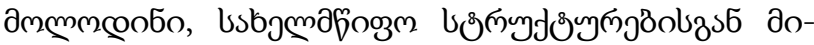
omml zsазпово

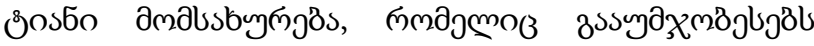

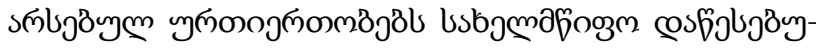

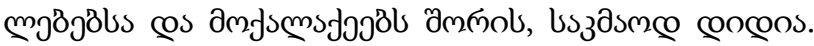

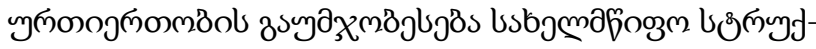

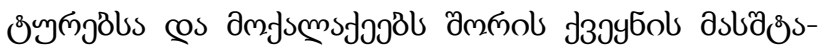

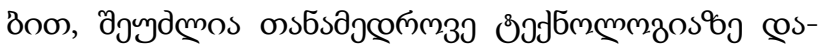

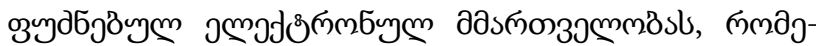

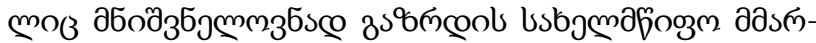

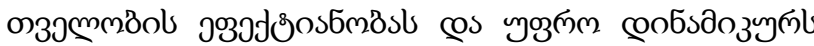

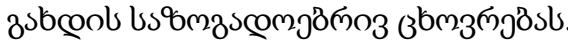

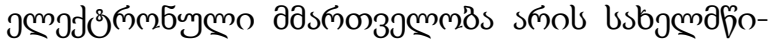

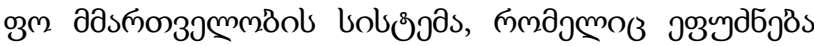

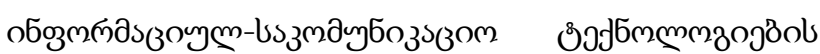

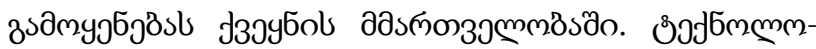

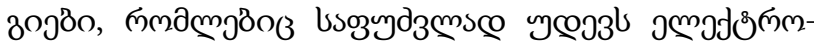

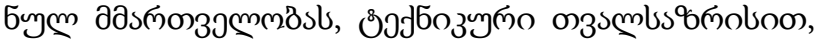




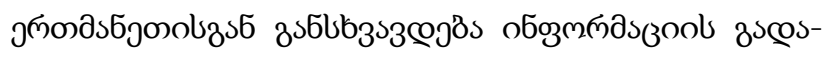

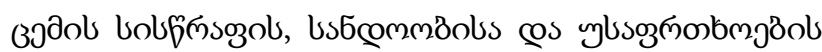

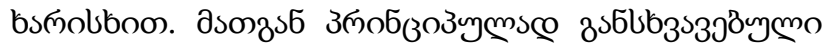

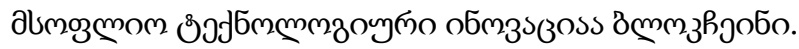

\section{domoosscon 5sfomo}

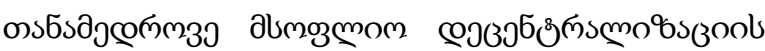

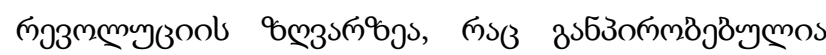

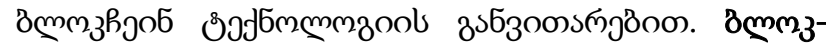

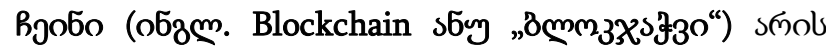

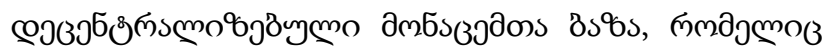

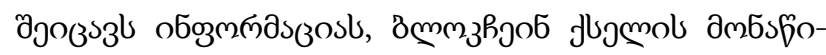

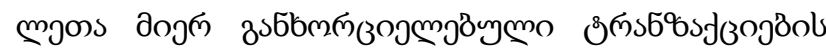

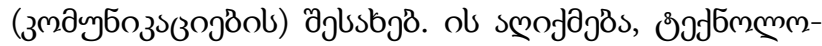

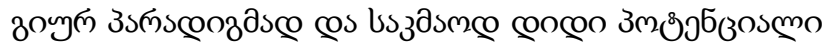

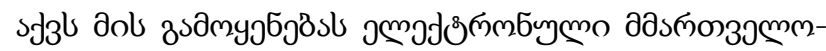

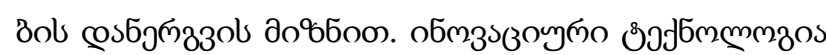

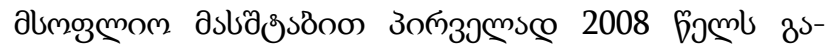

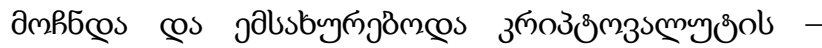

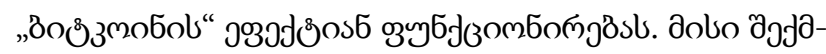

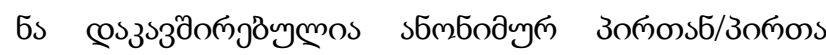

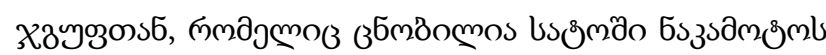
(Satoshi Nakamoto) bsbjmonon.

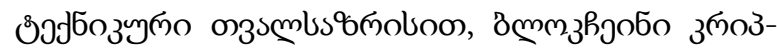

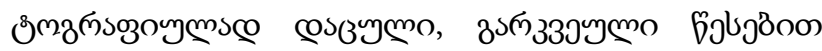

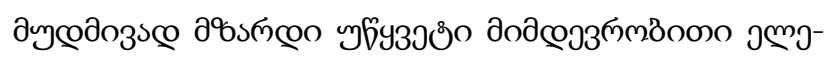
дృ бฮృ

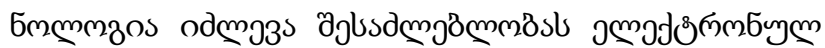

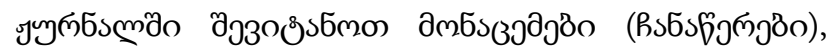

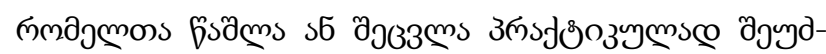

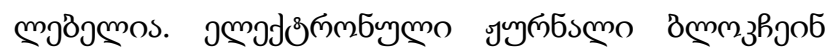

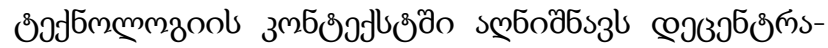

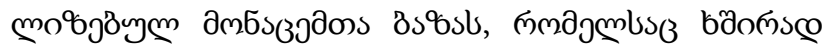

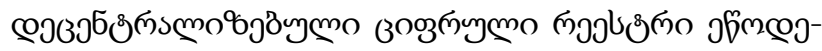

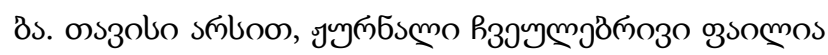

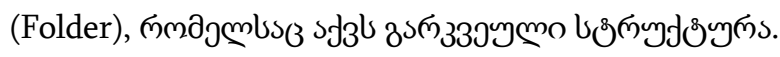

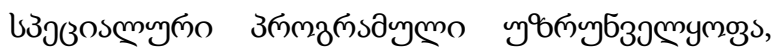

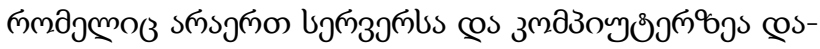

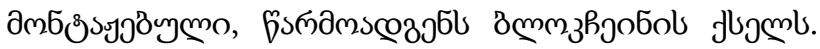

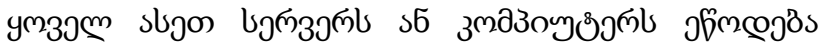

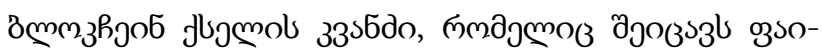

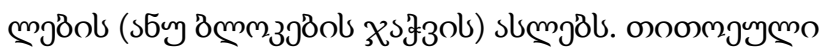

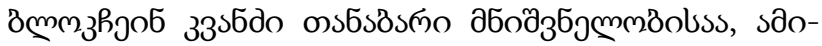

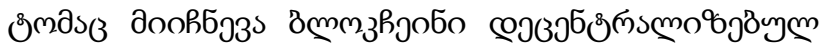

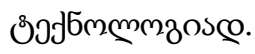

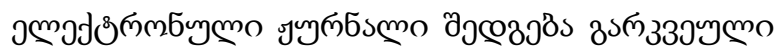

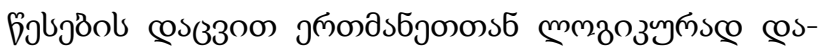

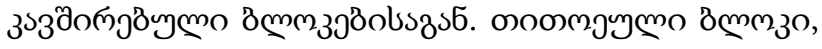

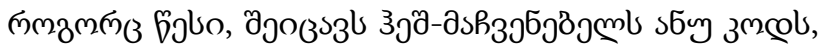

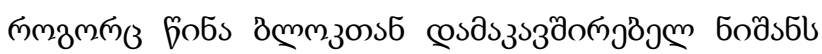

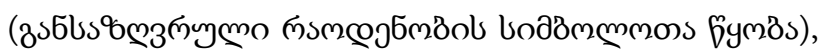

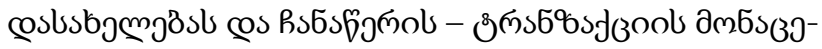

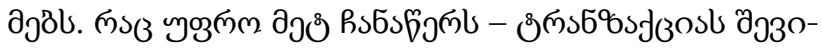

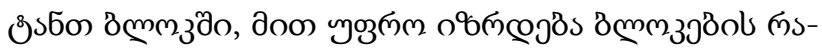

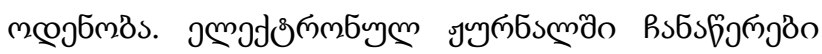

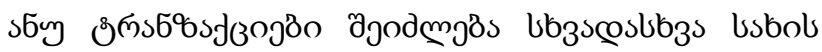

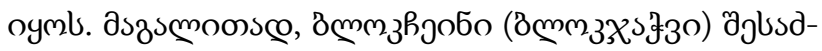

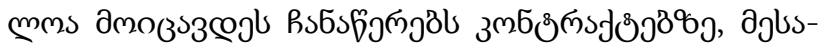

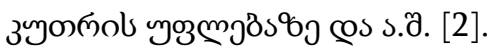

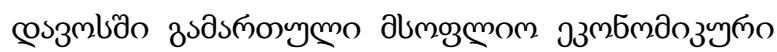

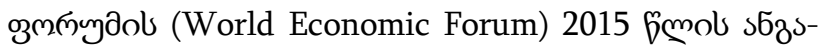

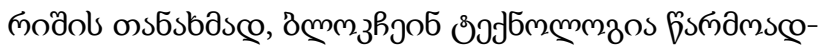

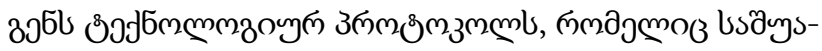

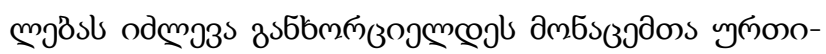

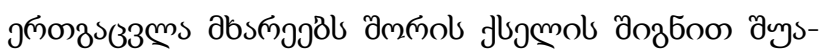

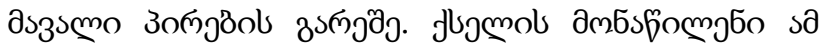

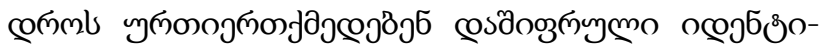

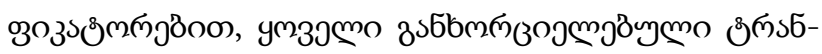

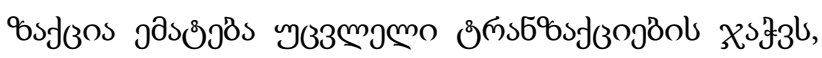

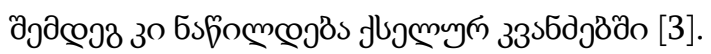




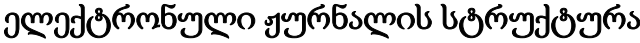

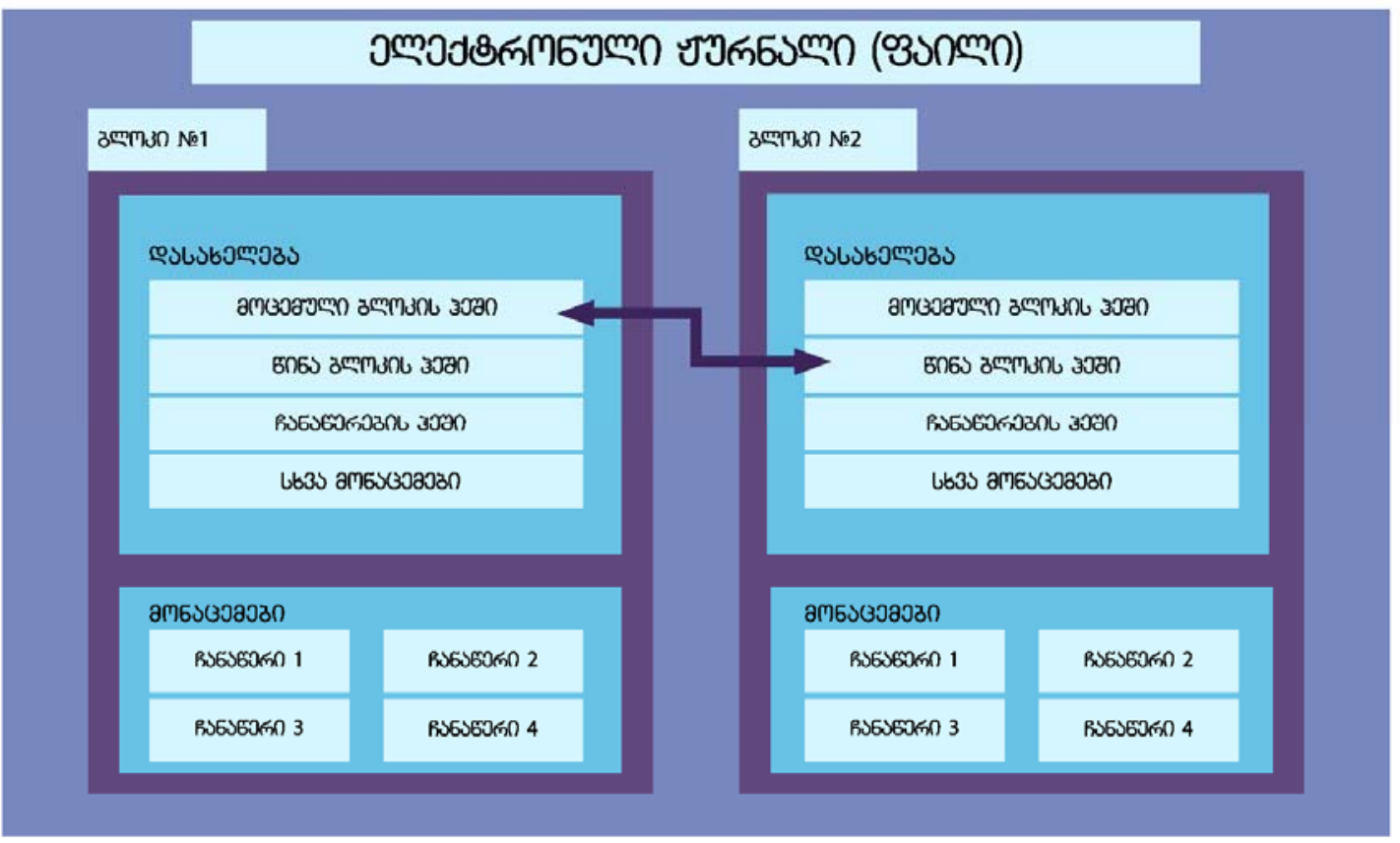

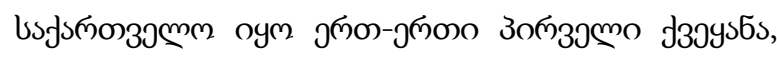

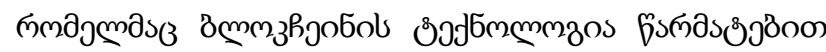

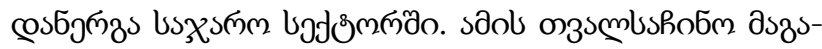

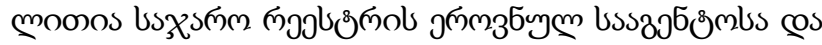

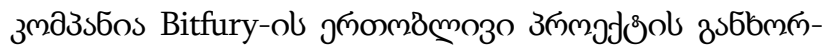

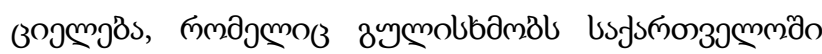

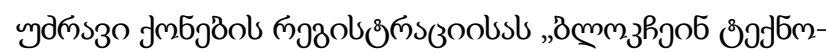

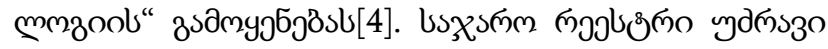

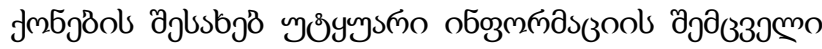

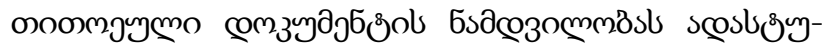

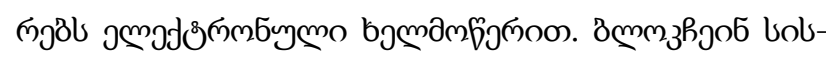

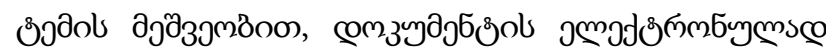

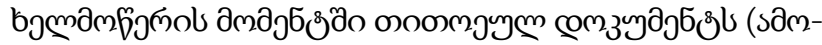

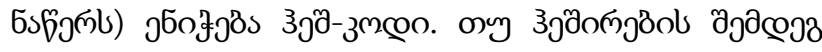

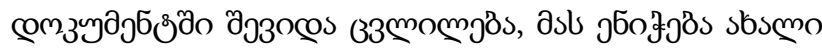

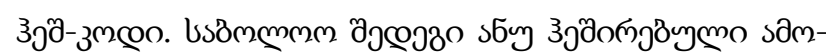

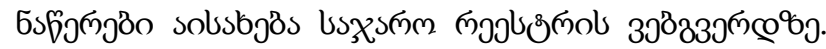

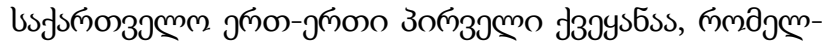

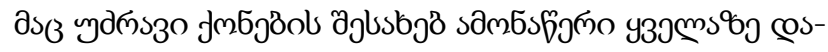

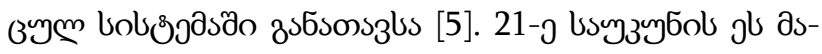

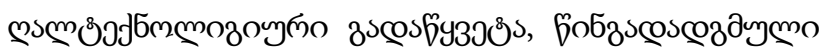

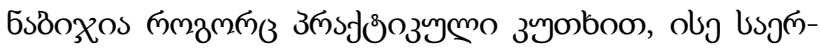

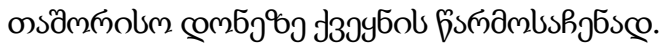

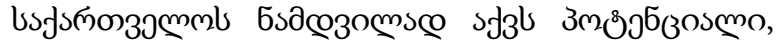

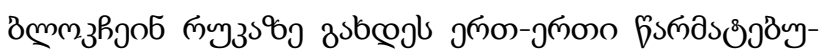

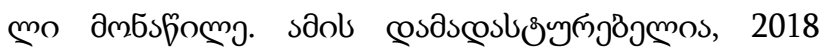

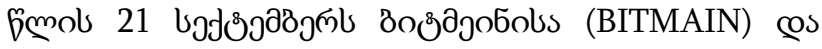

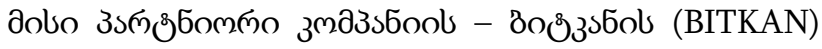

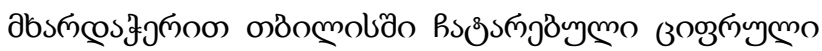

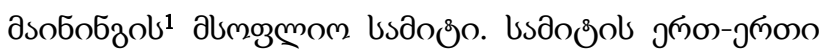

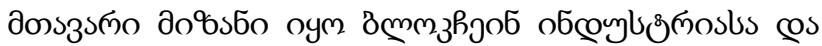

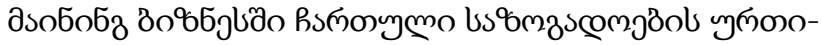

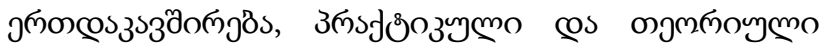

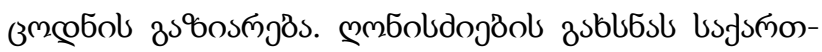

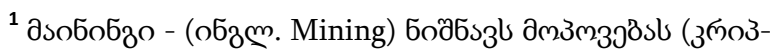

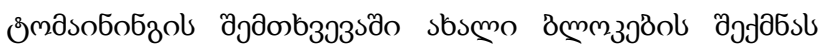

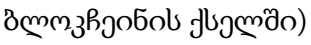
https://www.wordreference.com/enru/mining.
} 


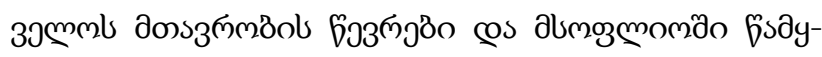

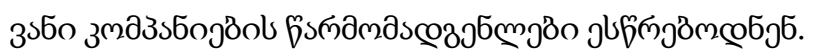

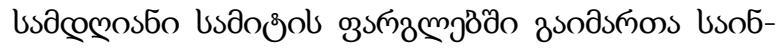

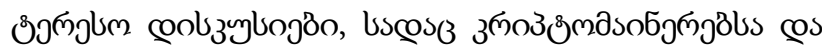

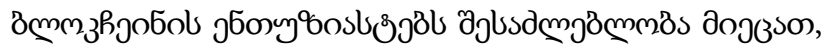

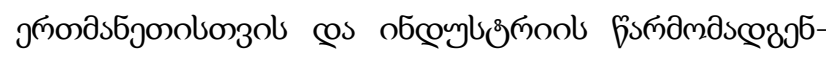

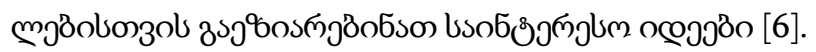

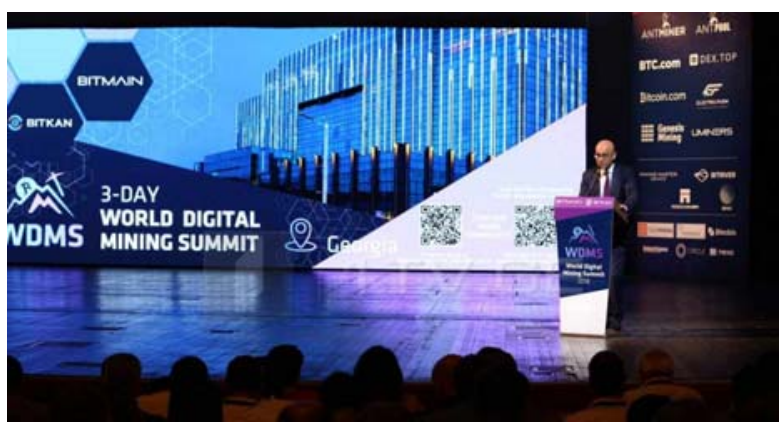

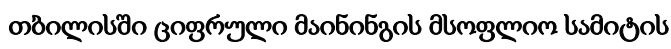
zublibs (21.09.2018) ${ }^{2}$

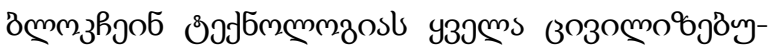

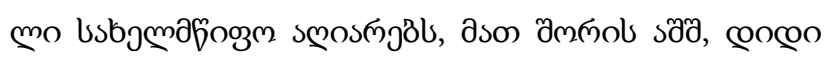

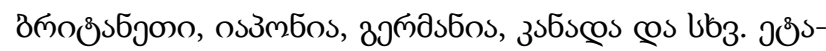

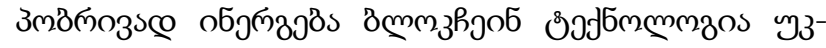

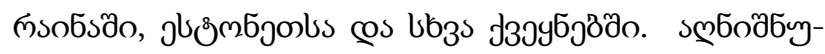

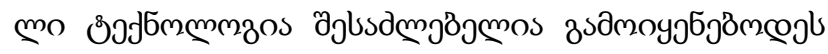

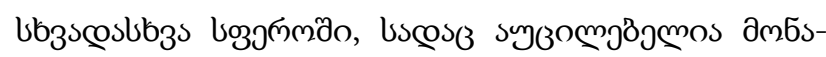

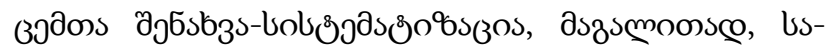

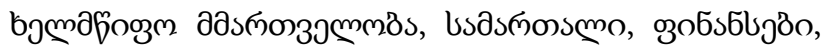

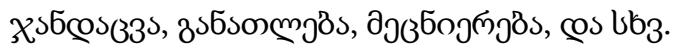

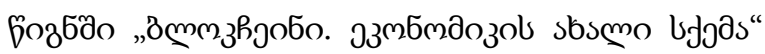
(Blockchain. Blueprint for a New Economy), 8̈mmз

${ }^{2}$ https://www.google.com/search?q=\%E1\%83\%AA\%E1\%8 3\%98\%E1\%83\%A4\%E1\%83\%A0\%E1\%83\%A3\%E1\%83\%9A \%E1\%83\%98+\%E1\%83\%9B\%E1\%83\%90\%E1\%83\%98\%E1\% 83\%9C\%Е1\%83\%98\%E1\%83\%9C\%E1\%83\%92\%E1\%83\%98 \%E1\%83\%A1+\%E1\%83\%A1\%E1\%83\%90\%E1\%83\%9B\%E1 \%83\%98\%E1\%83\%A2\%E1\%83\%98\&rlz=1C1KMZB_enGE57 7GE577\&source=lnms\&tbm $=$ isch\&sa $=X \& v e d=0 a h U K E w j p x n z$ dbgAhUKjosKHVRcDv8Q AUIDigB\&biw=1920\&bih=920

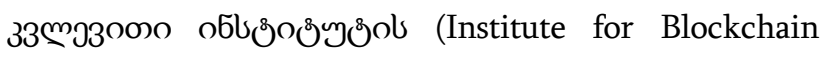

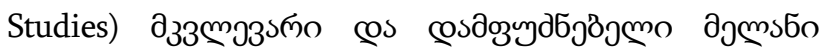

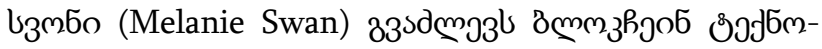

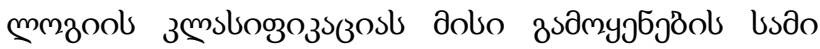

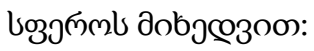

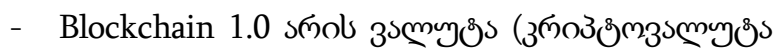

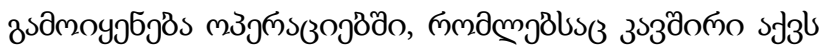

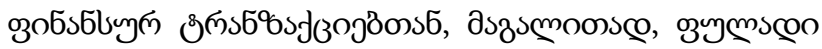

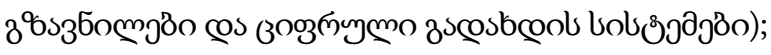

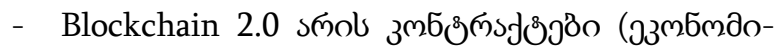

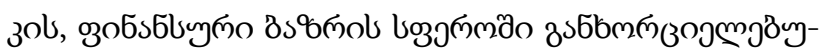

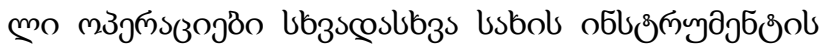

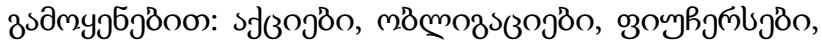

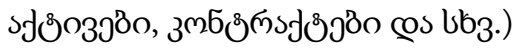

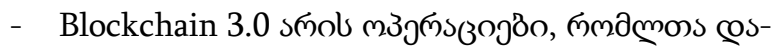

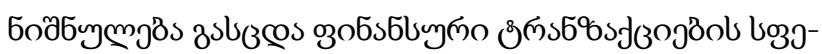

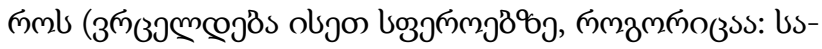

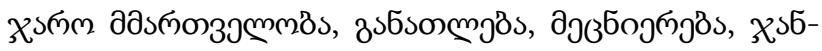
cos(33s cos bb 3.) [7].

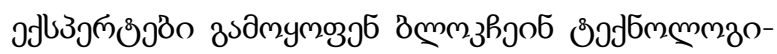

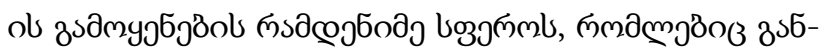

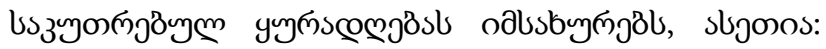
$[8,9,10]$

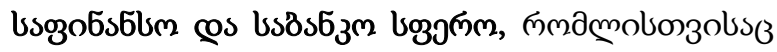

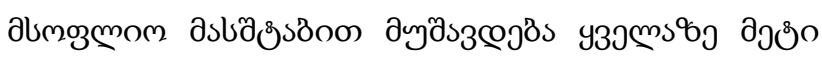

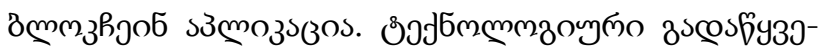

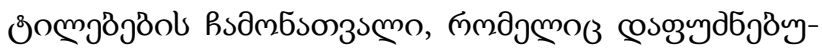

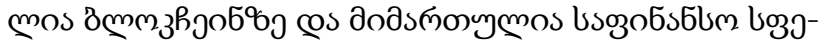

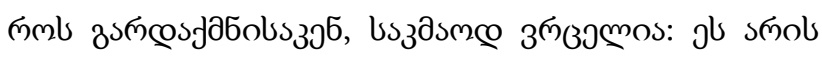

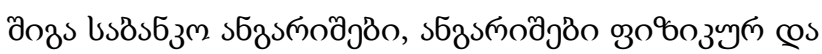

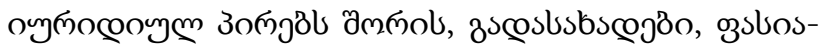

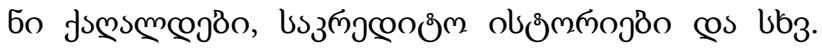

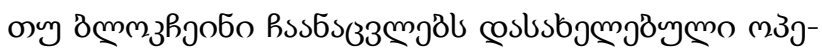

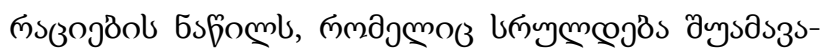




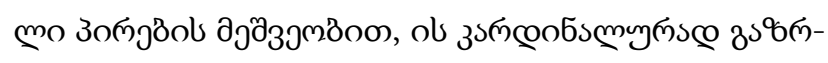

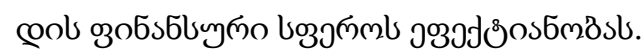

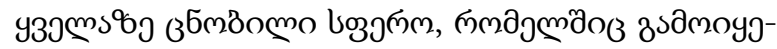

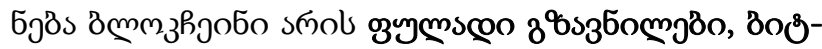

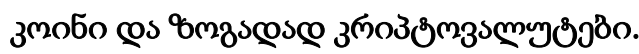

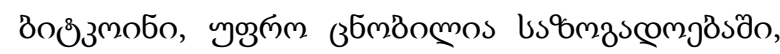

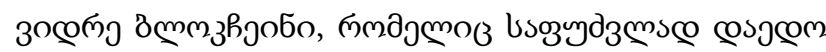

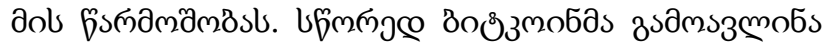

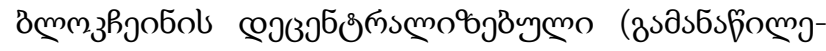

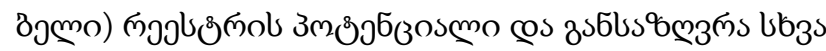

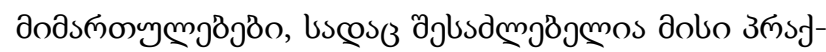

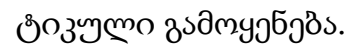

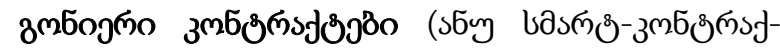

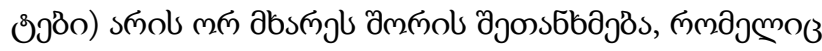

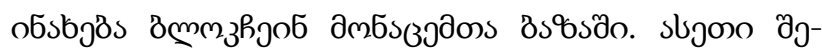

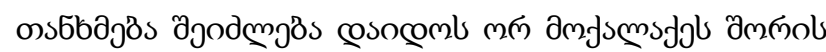
sбу peer-to-peer (P2P), amfs ms

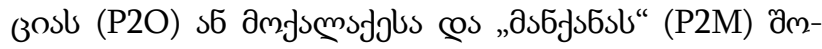

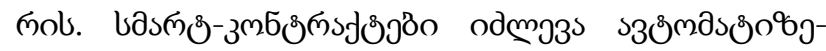

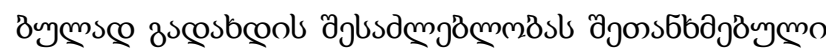

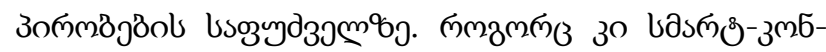

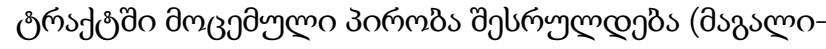

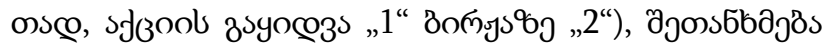

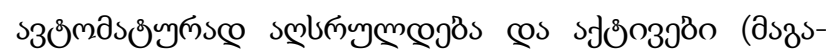

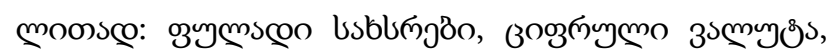

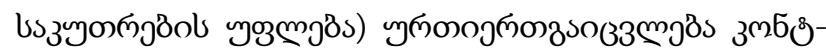

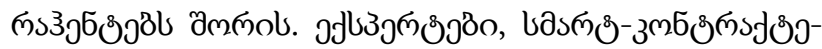

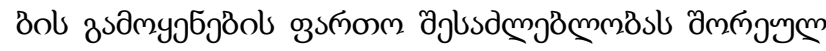

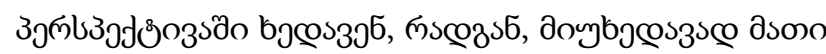

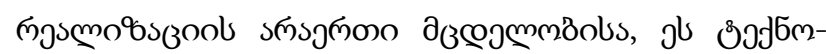

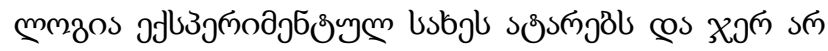

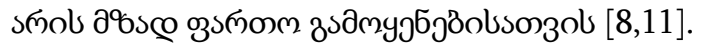

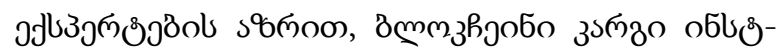

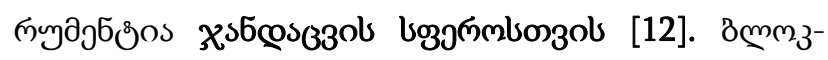

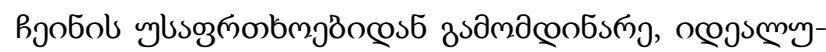

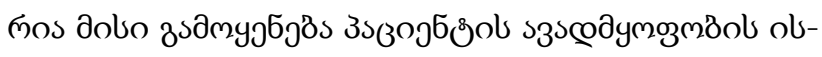

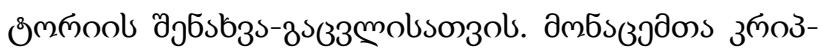

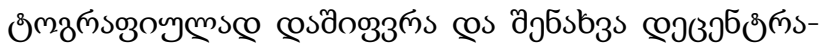

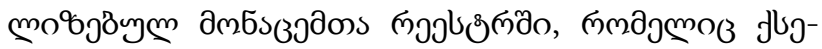

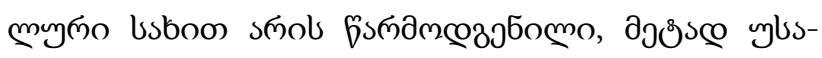

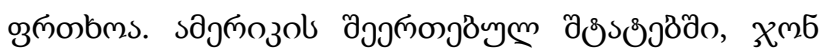

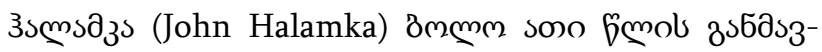

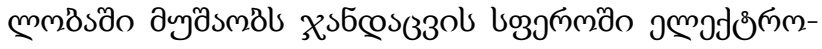

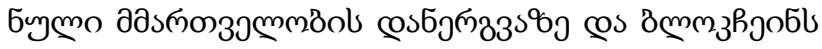

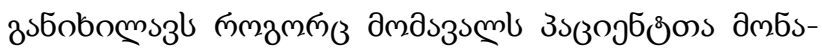

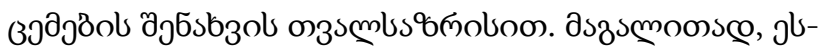

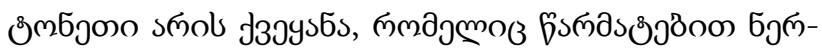

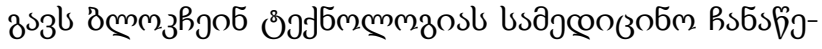

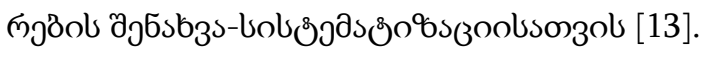

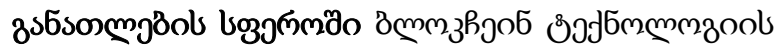

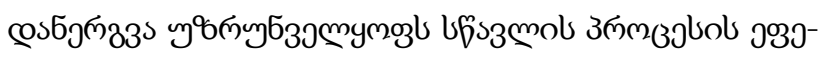

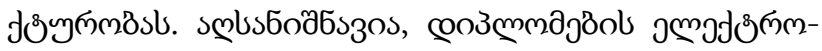

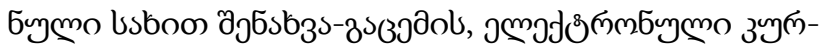

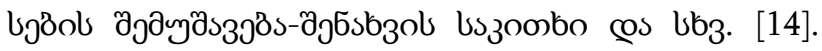

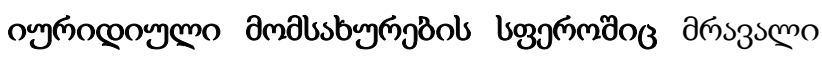

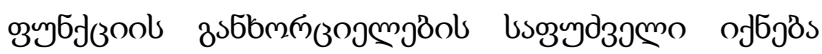

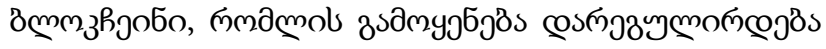

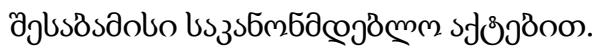

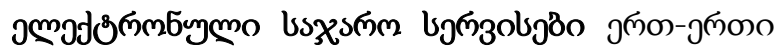

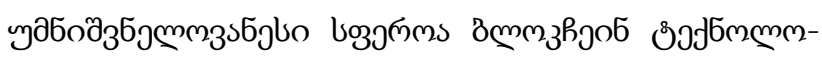

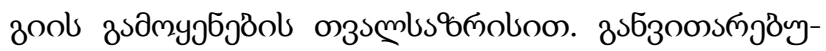

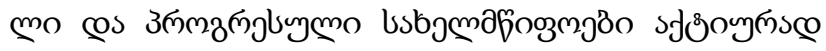

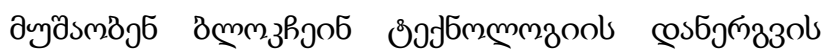

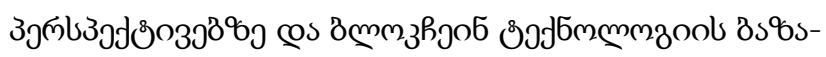

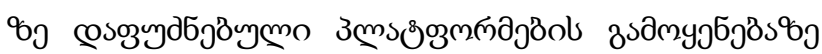

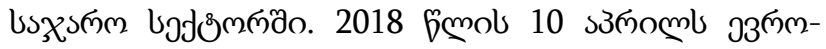

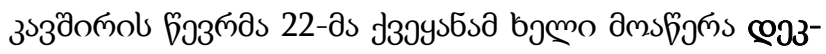

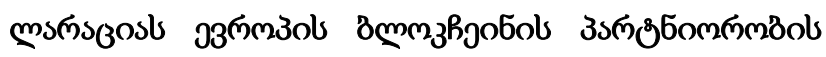

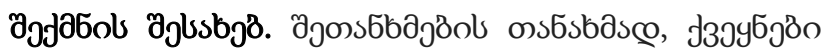

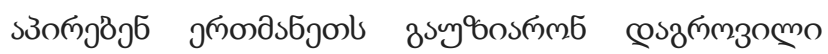




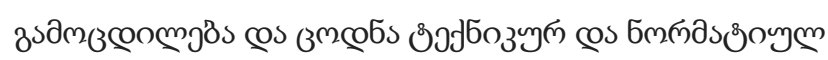

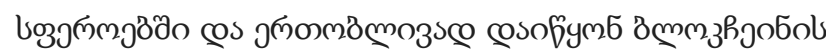

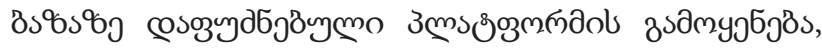

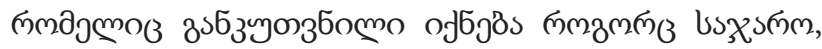

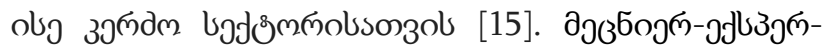

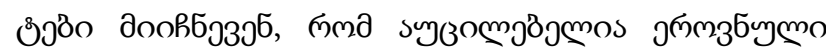

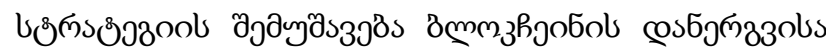

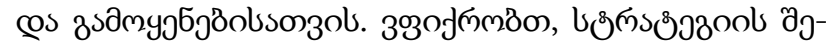

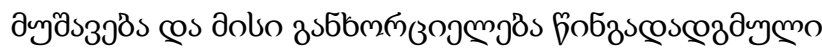

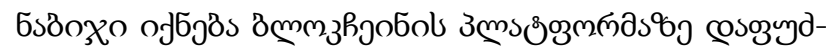

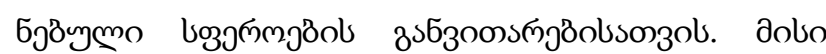

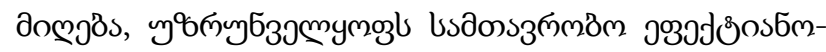

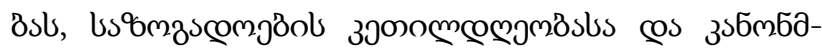

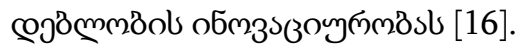

\section{cosb $33^{6 s}$}

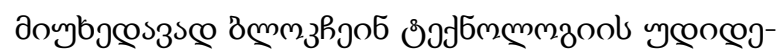

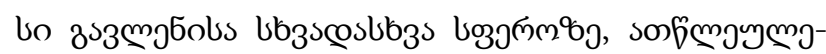
Bos bsfoпм odobsonzol, пма s̊

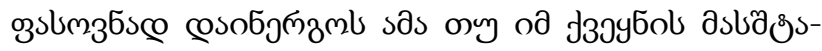

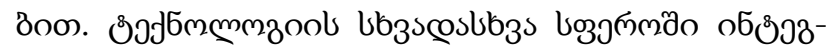

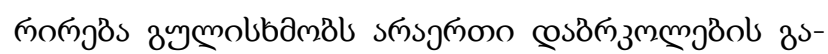

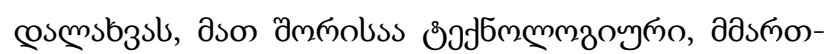

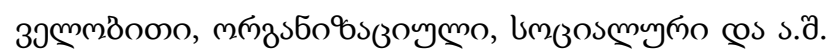

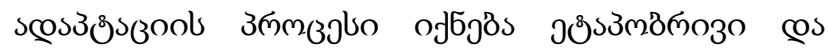

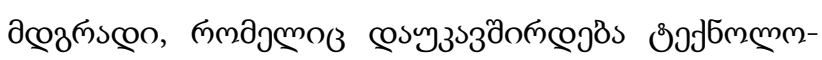

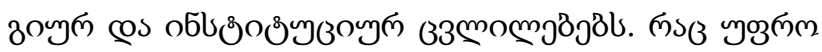

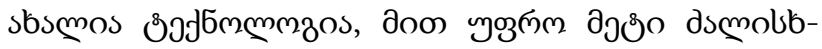

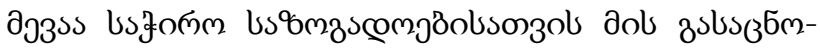

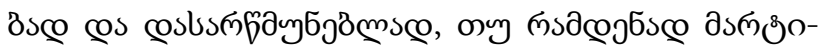

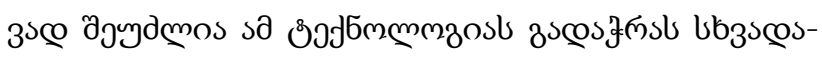

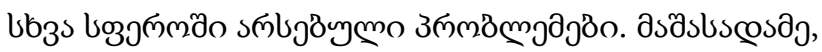

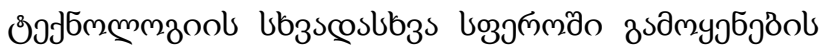

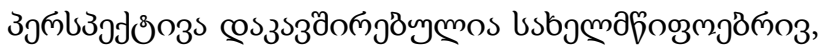

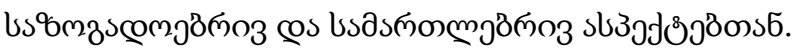

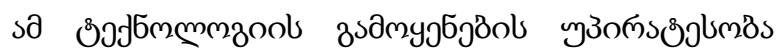

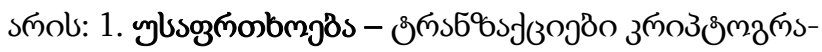

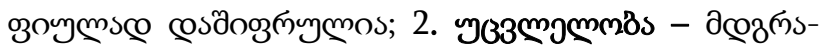

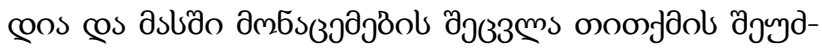

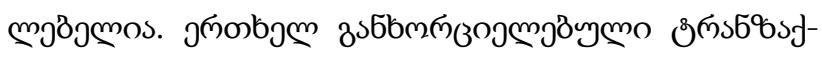

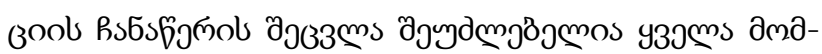

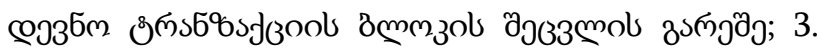

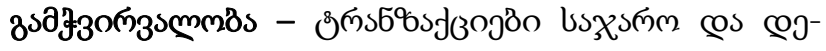
вэб

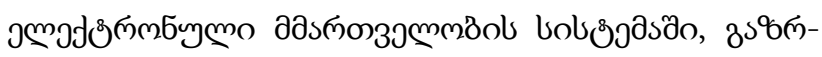

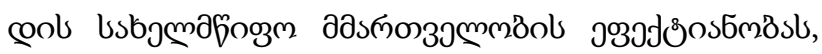

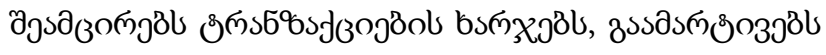

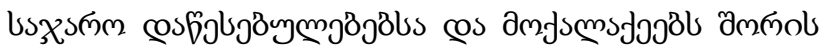

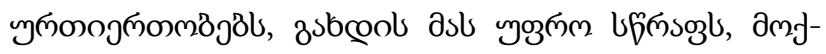
бomb cos jo3adతిosడl.

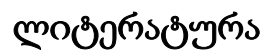

1. Genkin A., Mikheev A. Blockchain - how it works and what awaits us tomorrow. M.: Alpina Publisher. 2018, 148 p. (in Russian).

2. URL: https://ruhash.com/chajnikam/blokchejn-dlya-novichkov-obshhie-predstavleniya-o-tehnologii.html (in Russian).

3. URL: http://www3.weforum.org/docs/WEF_GAC15_Technological_Tipping_Points_report_2015.pdf\#page=24

4. URL: https://ipress.ge/new/sajaro-reestri-inovatsiur (in Georgian).

5. URL: https://medium.com

6. URL: https://mybitcoin.ge/2018/09/03/bitmain (in Georgian).

7. Swan M. Blockchain: Blueprint for a new economy. 2015. 
8. Iansiti M., Lakhani K. R. The truth about blockchain. Harvard Business Review. 2017, 118-127 pp.

9. Tech Trends 2016: Innovation in the digital era.

URL: https://www2.deloitte.com/content/dam/Deloitte/global/Documents/Technology/gx-tech-trends-2016-innovating-digital-era.pdf

10. URL: https://mitsloan.mit.edu/ideas-made-to-matter/blockchain-explained

11. URL: https://www.forbes.ru/tehnologii/343843-programmy-blokcheyna-chto-prepyatstvuet-massovomuvnedreniyu-smart-kontraktov (in Russian).

12. URL: https://www.prnewswire.com/news-releases/blockchain-adoption-moving-rapidly-in-banking-andfinancial-markets-some-65-percent-of-surveyed-banks-expect-to-be-in-production-in-three-years300335472.html

13. URL: https://zudu.co.uk/blog/blockchain-technology-industry-applications/

14. URL: https://prostocoin.com/blog/blockchain-education (in Russian).

15. Jun M. Blockchain government: A next form of infrastructure for the twenty-first century. Journal of open innovation: Technology, market, and complexity. 2018.

16. Kandelaki N. Blockchain technology and its future in e-Government. The science journal "Authority and society (history, theory, practice)”. №1 (49). Tbilisi. 2019. (in Georgian).

UDC 621.385

SCOPUS CODE 1405

DOI: HTTPS://DOI.ORG/10.36073/1512-0996-2019-3-11-18

\title{
Prospects for the use of blockchain technology in e-Government
}

Natalia Kandelaki

\author{
Department of Business Administration, Georgian Technical University, 77 M. Kostava \\ str., 0160 Tbilisi, Georgia \\ E-mail: nataliakandelaki89@gmail.com
}

\section{Reviewers:}

G. Iashvili, Professor, Faculty of Business Technology, GTU

E-mail: genadi_iashvili@hotmail.com

S. Dogonadze, Professor, Faculty of Business Technology, GTU

E-mail: shotad55@gmail.com

Abstract. One of the most important conditions for the development of the country is the concept of effective, expeditious and flexible management. Therefore, in this direction it is very important to develop e-Government and updated e-Government policy.

The prospect of using blockchain technology in e-Government represents actually interesting issue for Georgia. The article briefly discusses the essence of the revolutionary-innovative technology, principles of work performance and the scope of use, analyzes the feasibility of integrating technology into the electronic control system.

Key words: Blockchain; e-Government; electronic services; technology. 
UDC 621.385

SCOPUS CODE 1405

DOI: HTTPS://DOI.ORG/10.36073/1512-0996-2019-3-11-18

\section{Перспективы использования блокчейн-технологии в электронном управлении}

Наталья Канделаки Департамент публичного управления и электронного бизнеса, Грузинский технический университет, Грузия, 0160, Тбилиси, ул. М. Костава, 77

E-mail: nataliakandelaki89@gmail.com

\section{Рецензенты:}

Г. Яшвили, профессор факультета бизнес-технологий ГТУ

E-mail: genadi_iashvili@hotmail.com

Ш. Догонадзе, профессор факультета бизнес-технологий ГТУ

E-mail: shotad55@gmail.com

Аннотация. Одним из важнейших условий развития страны является концепция эффективного, оперативного и гибкого управления. Поэтому в этом направлении очень важно развивать электронное управление и разрабатывать обновленную политику электронного управления. Перспектива использования блокчейн-технологии в электронном управлении для Грузии является интересным и актуальным вопросом. В статье кратко рассматривается суть революционно-инновационной технологии, принципы ее работы и сферы использования, проанализирована целесообразность интеграции технологии в систему электронного управления.

Ключевые слова: блокчейн; технологии; электронное управление; электронные услуги.

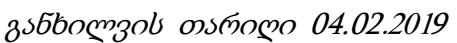

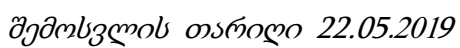

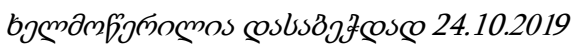

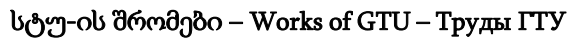


UDC 666.953

SCOPUS CODE 1508

DOI: https://doi.org/10.36073/1512-0996-2019-3-19-28

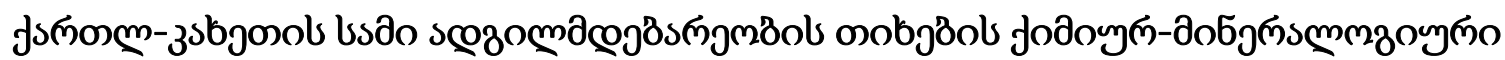

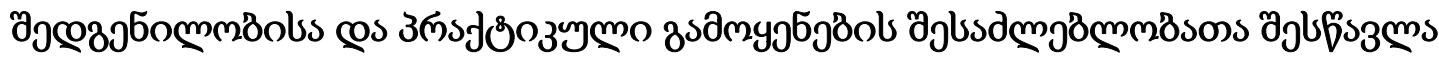

зо3о mmensdo

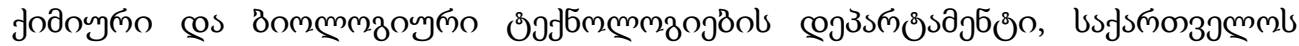

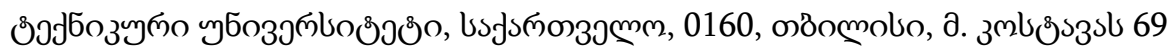

E-mail: g.loladze@gtu.ge

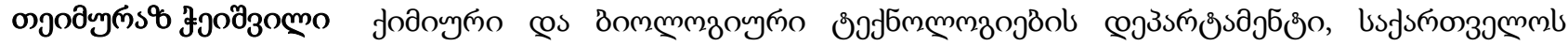

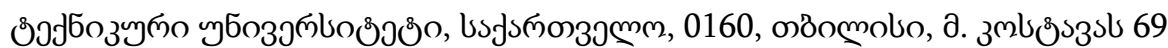

E-mail: t.cheishvili@gtu.ge

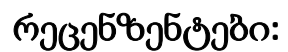

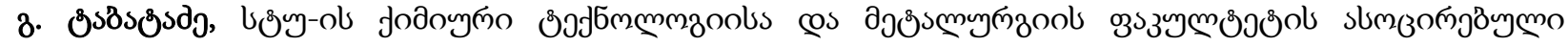
Зпмозјымпо

E-mail: g.tabatadze@yahoo.com

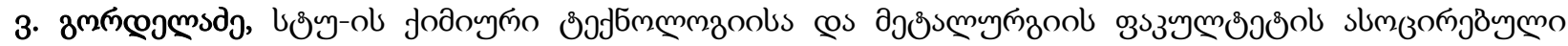

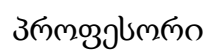

E-mail: b.gordeladze@gtu.ge

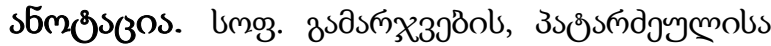

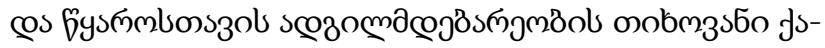

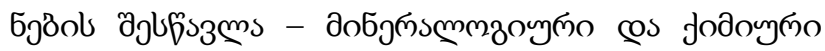

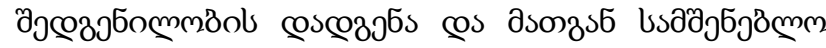

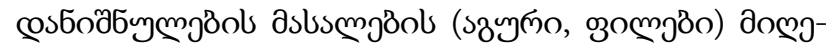

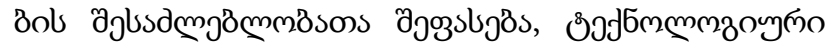

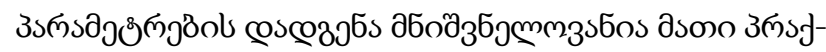

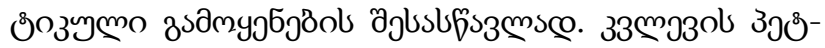

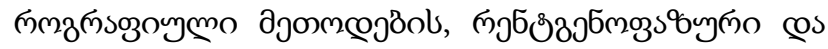

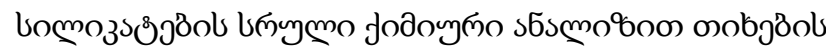

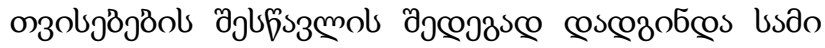

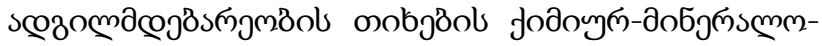

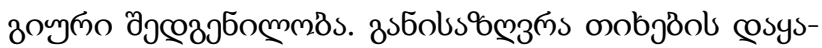

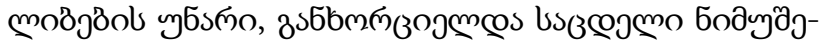

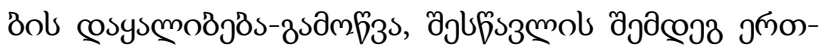

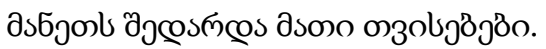

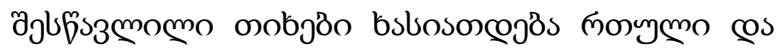

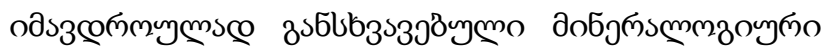

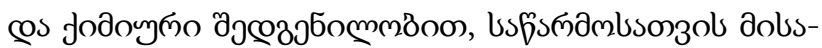

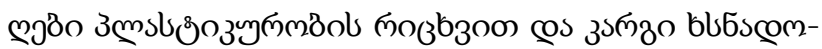

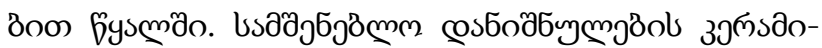

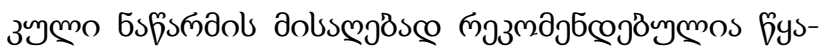

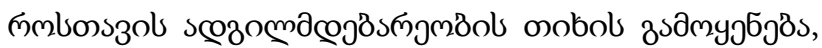




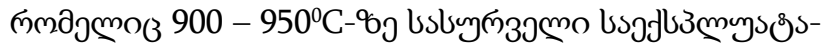

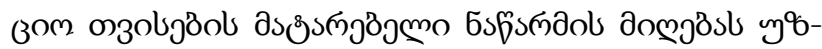

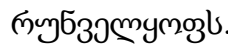

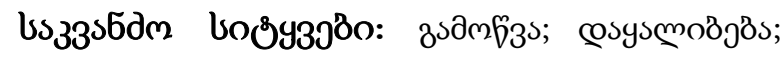

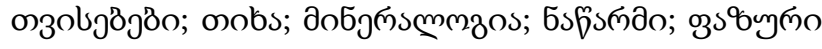

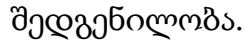

\section{gjuszsmo}

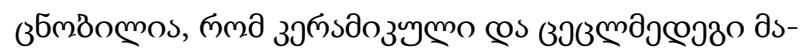

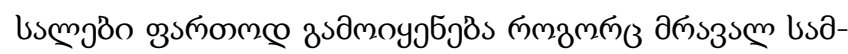

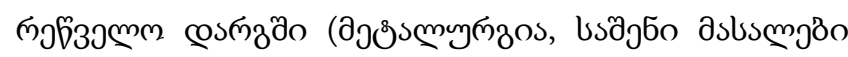

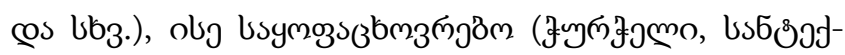

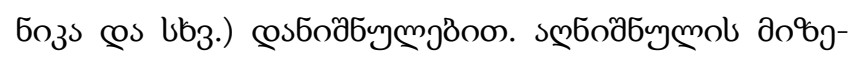

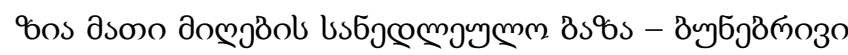

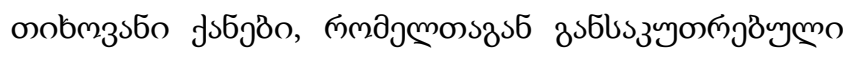

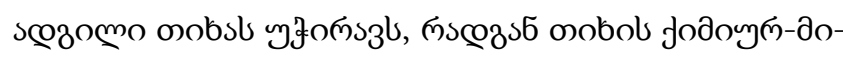

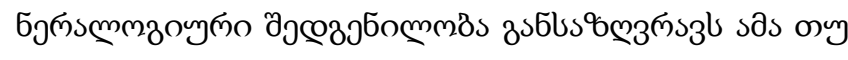

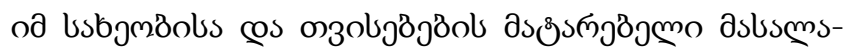

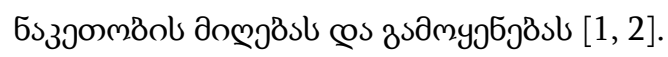

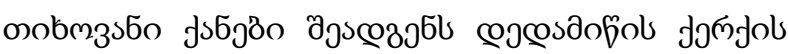

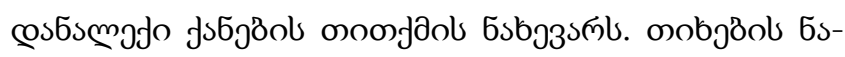

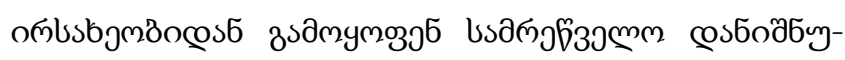

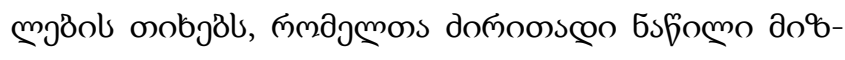

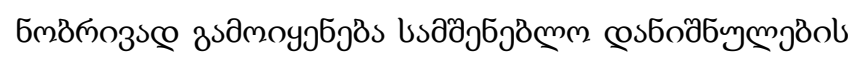

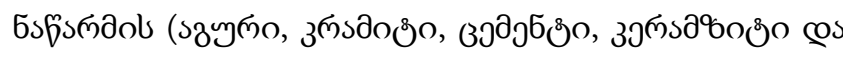

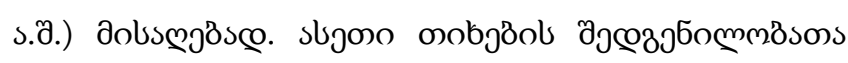

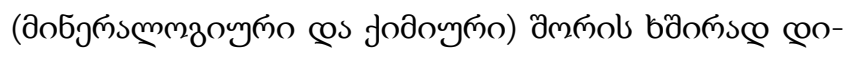

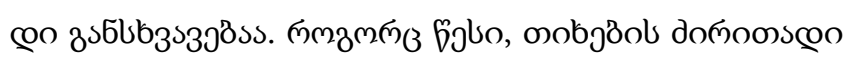

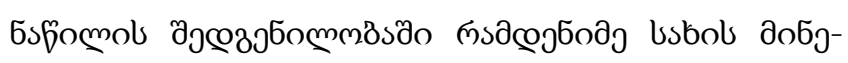

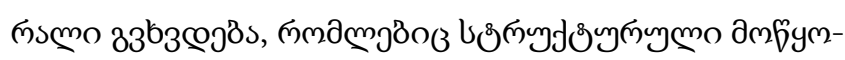

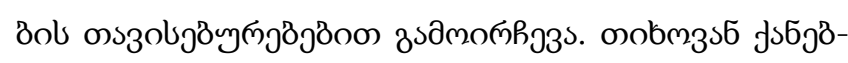

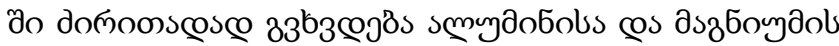

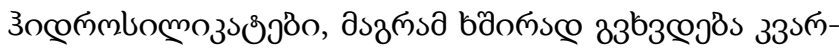

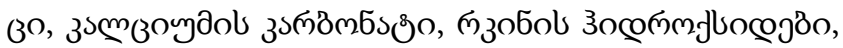

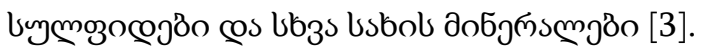

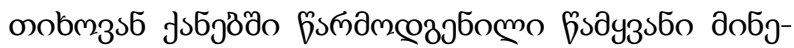

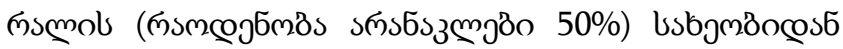

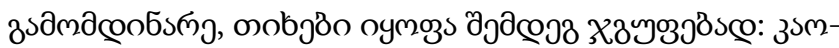

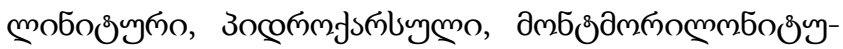

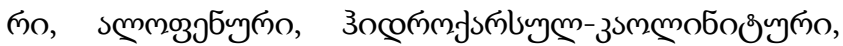

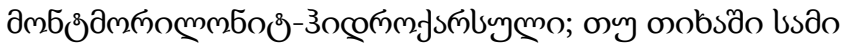

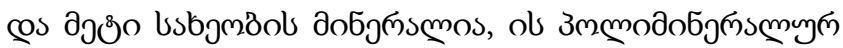

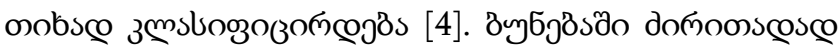

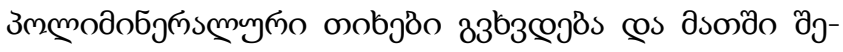

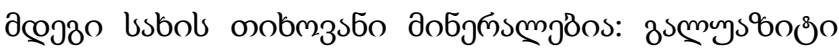

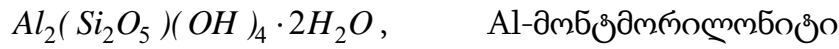

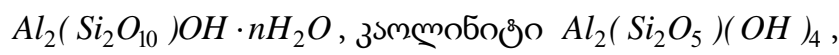

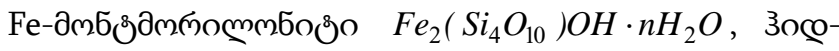

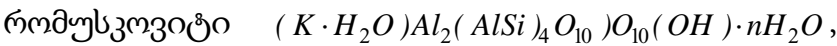

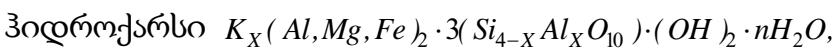
sммmo3j5o $\mathrm{pAl}_{2} \mathrm{O}_{3} \cdot q \mathrm{SiO}_{2} \cdot n \mathrm{H}_{2} \mathrm{O}$, bscosos $\mathrm{X} \leq 0,5$ cos $\mathrm{n}$ $\leq 1,5$ cos bb 3 s [5-7].

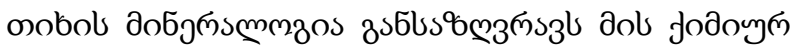

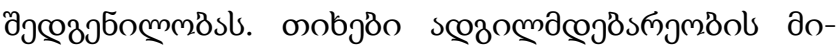

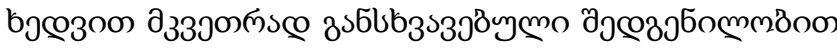
дзамопяэз (asl. \%) : 42 - $75 \mathrm{SiO}_{2}, 7$ - $23 \mathrm{Al}_{2} \mathrm{O}_{3}, 12-$

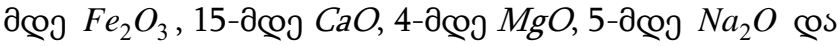

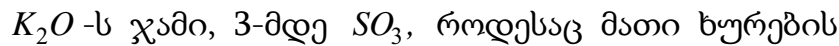

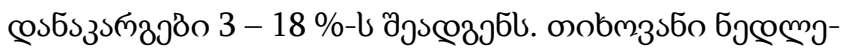

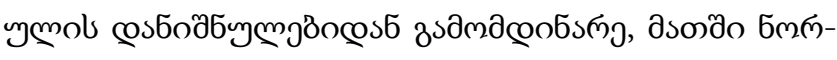

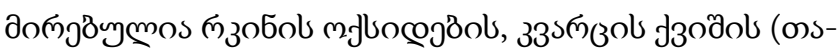

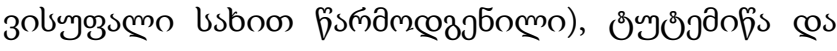

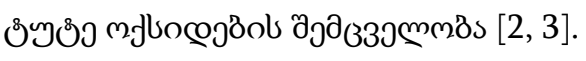




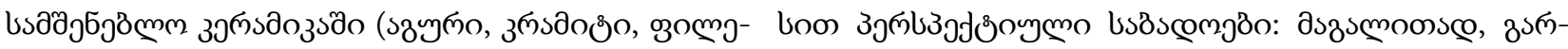

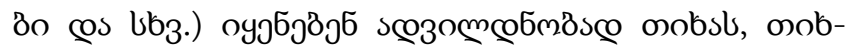

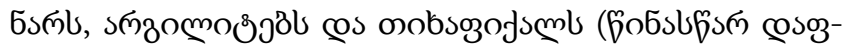

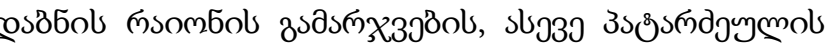

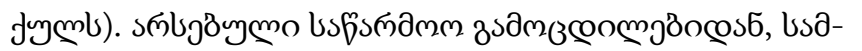

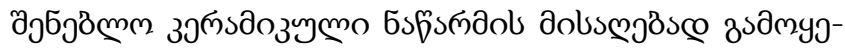

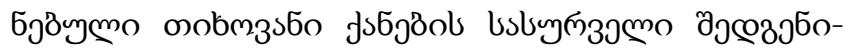
mmß̊ss (6ீрб. \%): 53-81 $\mathrm{SiO}_{2}, 7-23\left(\mathrm{Al}_{2} \mathrm{O}_{3}+\mathrm{TiO}_{2}\right), 2-$

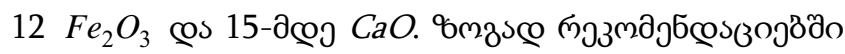

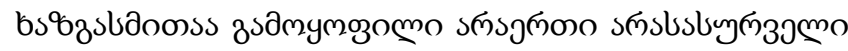

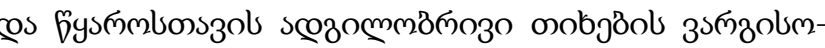

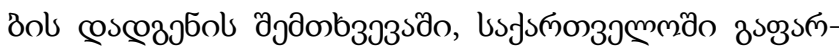

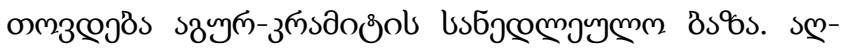

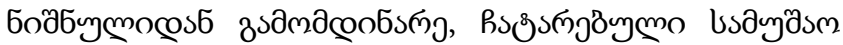

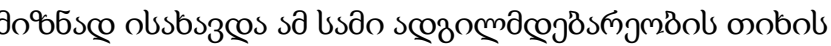

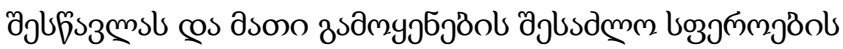
coscososts.

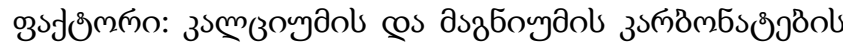

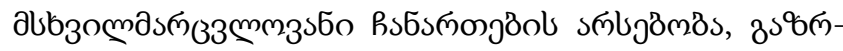

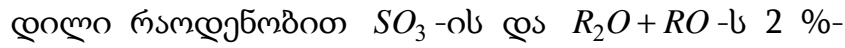

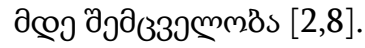

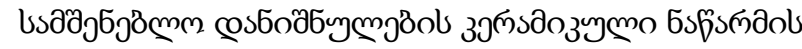

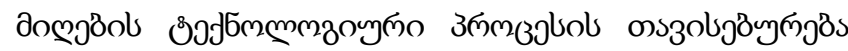

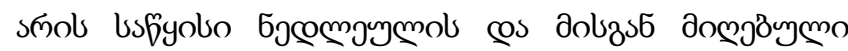

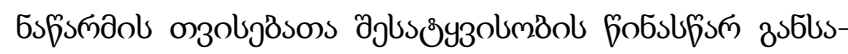

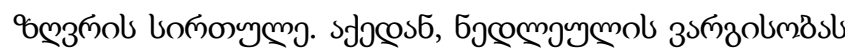

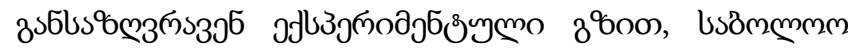

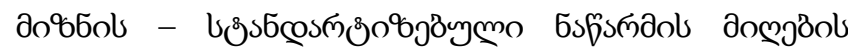

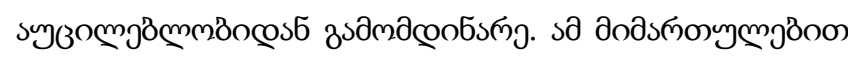

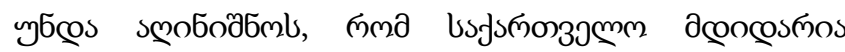

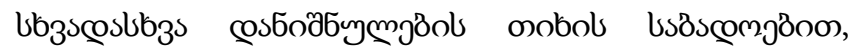

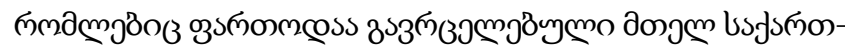

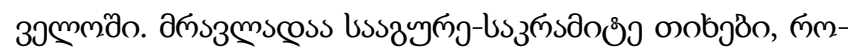

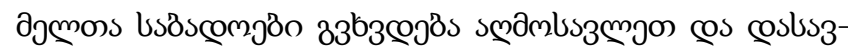

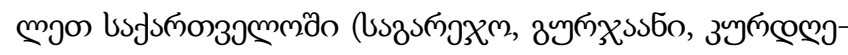

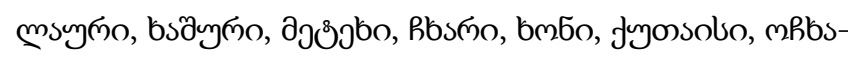

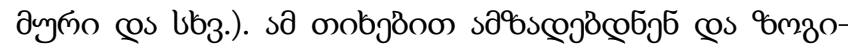

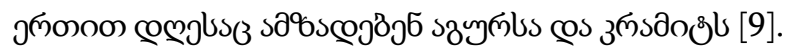

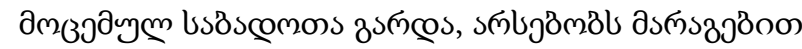

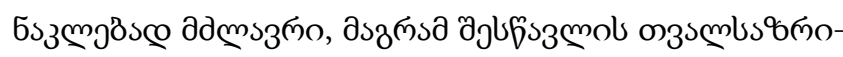

\section{donnoosen 6sfomo}

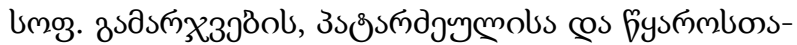

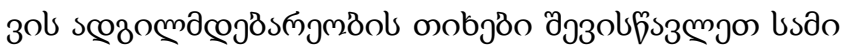

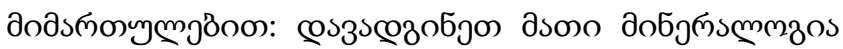

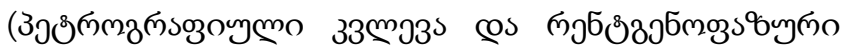

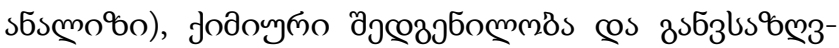

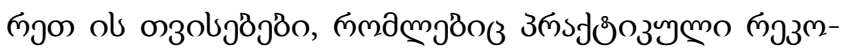

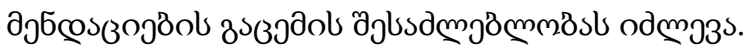

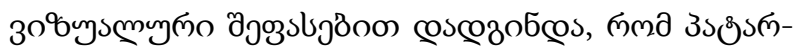

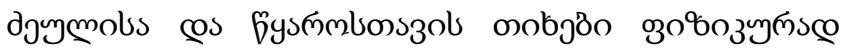

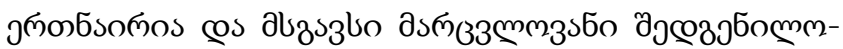

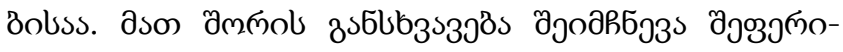

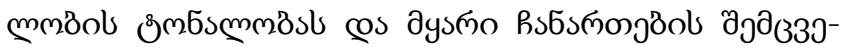

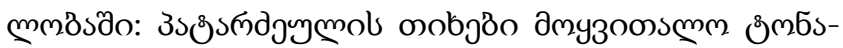

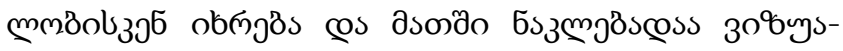

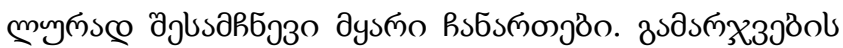

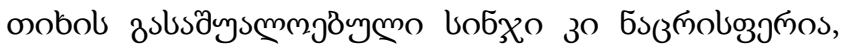

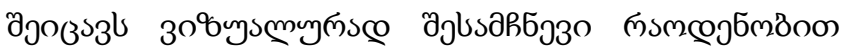

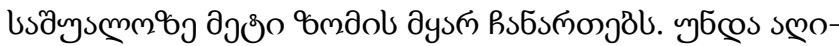

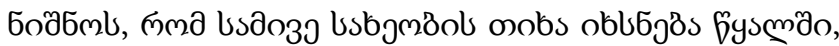

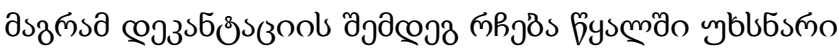

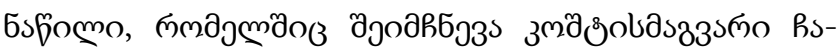

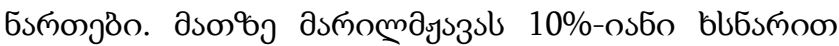




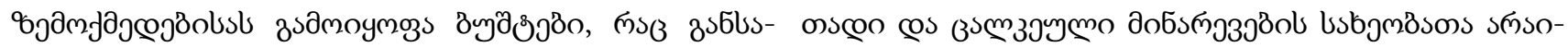

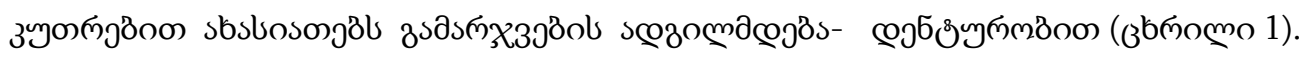

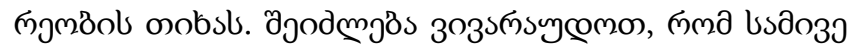

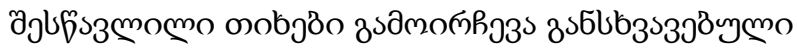

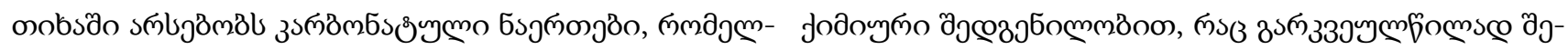

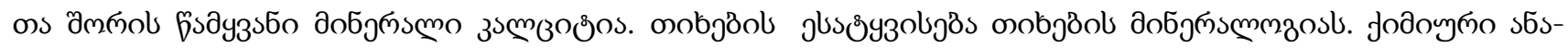

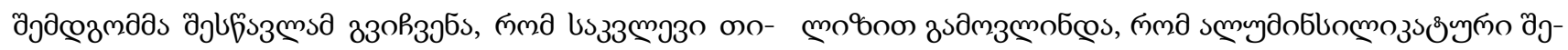

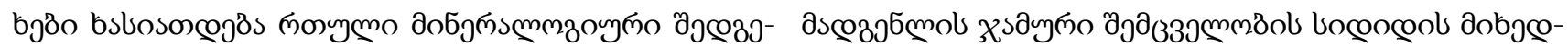

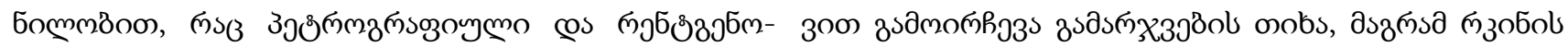

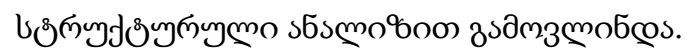

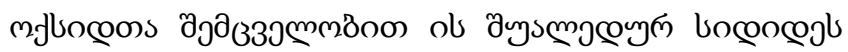

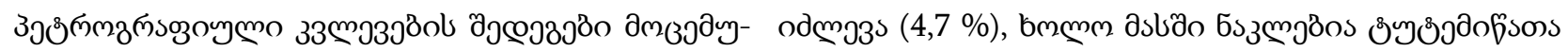

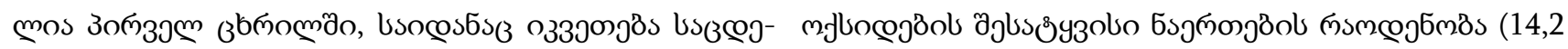

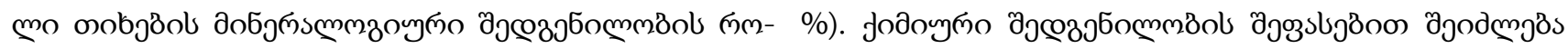

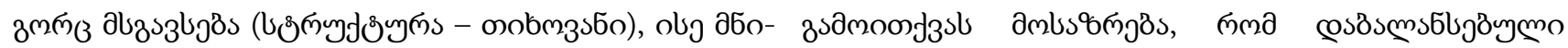

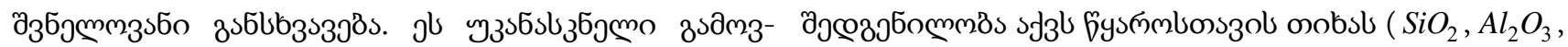

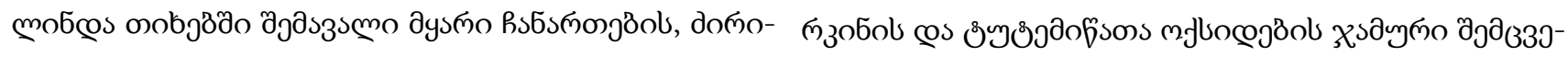
mmöol dobjœзоo).

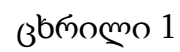

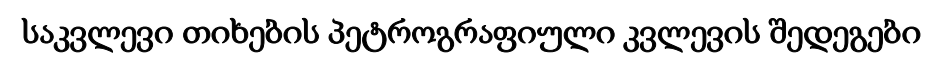

\begin{tabular}{|c|c|c|c|c|c|}
\hline $\mathrm{N}$ & 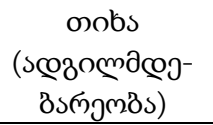 & 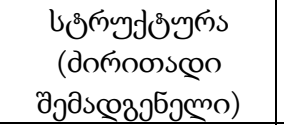 & 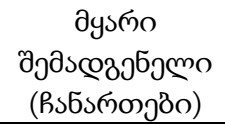 & 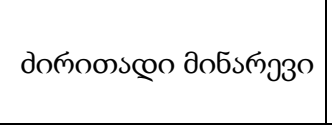 & 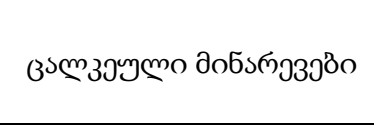 \\
\hline 1 & 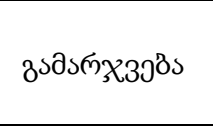 & 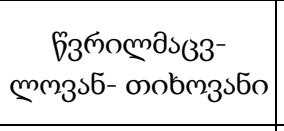 & 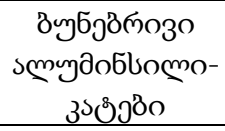 & 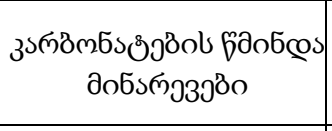 & 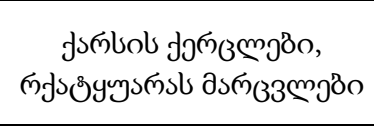 \\
\hline 2 & 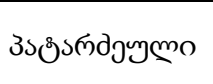 & oоbm3sбo (д‡mg) & 3 & 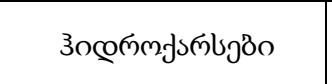 & 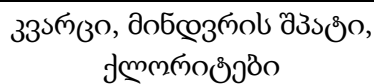 \\
\hline 3 & 6ysলmborszo & oоbm3sбo (дłmı) & 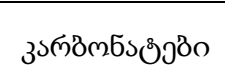 & 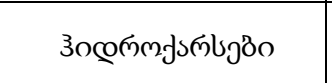 & 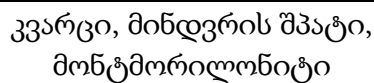 \\
\hline
\end{tabular}

$3_{\text {b̆омо } 2}$

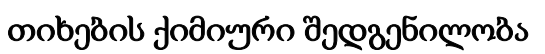

\begin{tabular}{|c|c|c|c|c|c|c|c|c|c|c|c|}
\hline \multirow[b]{2}{*}{ 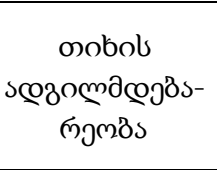 } & \multicolumn{11}{|c|}{ 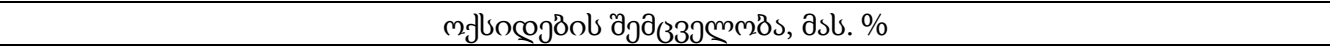 } \\
\hline & $\mathrm{SiO}_{2}$ & $\mathrm{Al}_{2} \mathrm{O}_{3}$ & $\begin{array}{l}\mathrm{FeO}+ \\
\mathrm{Fe}_{2} \mathrm{O}_{3}\end{array}$ & $\begin{array}{c}\mathrm{CaO}+ \\
\mathrm{MgO}\end{array}$ & $\begin{array}{l}\mathrm{Na}_{2} \mathrm{O}+ \\
\mathrm{K}_{2} \mathrm{O}\end{array}$ & $\mathrm{SO}_{3}$ & $\mathrm{TiO}_{2}$ & $\mathrm{P}_{2} \mathrm{O}_{5}$ & $\mathrm{MnO}$ & $\begin{array}{l}\text { uobx. } \\
\text { uobjl- } \\
\text { ฮీग }\end{array}$ & b.œ. - \\
\hline 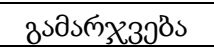 & 50,2 & 14,9 & 4,7 & 14,2 & 2,3 & 0,2 & 0,7 & - & - & 2,1 & 10,7 \\
\hline 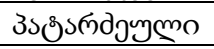 & 44,9 & 13,1 & 5,0 & 16,8 & 2,2 & 0,2 & 0,6 & 0,1 & 0,1 & 2,3 & 14,7 \\
\hline fysmmlonszo & 47,2 & 12,7 & 3,8 & 15,7 & 2,4 & - & 0,8 & 0,2 & 0,2 & 2,6 & 14,4 \\
\hline
\end{tabular}




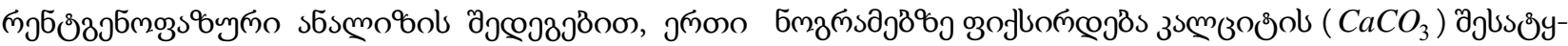

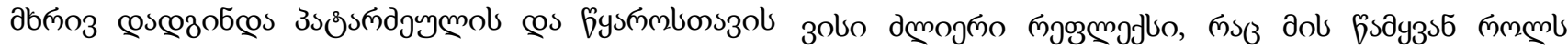

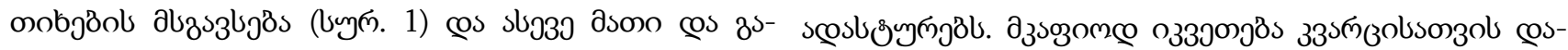

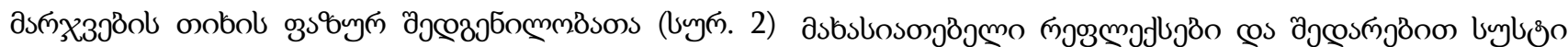

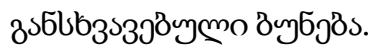

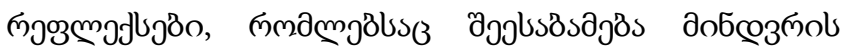

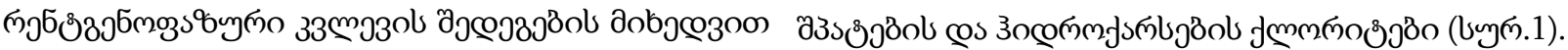

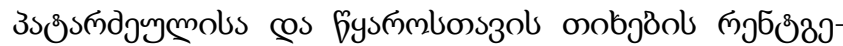

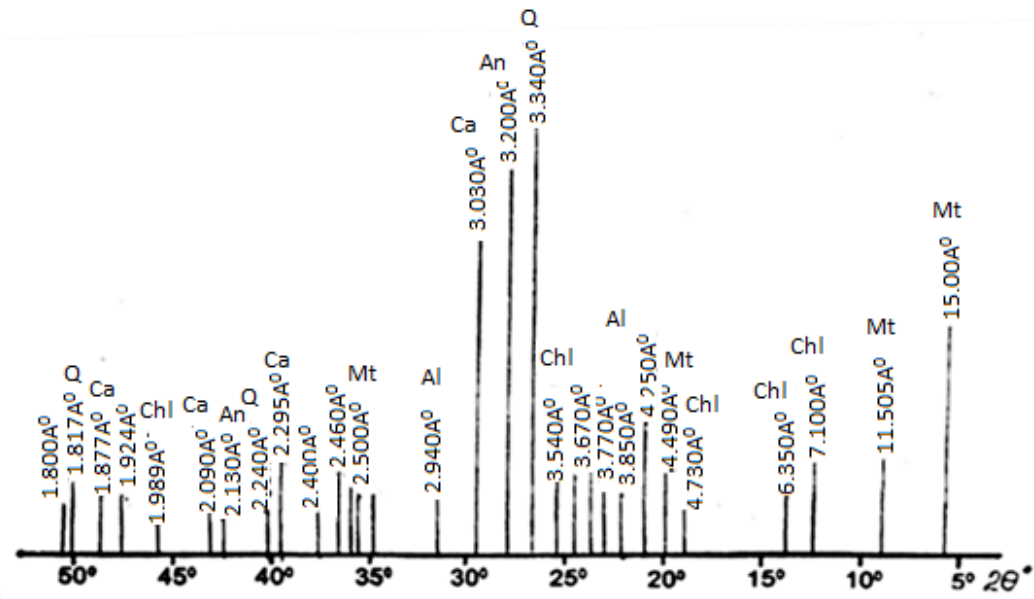

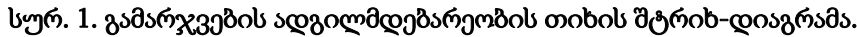

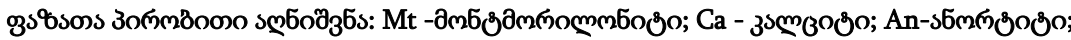

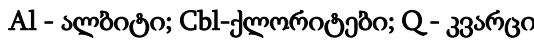
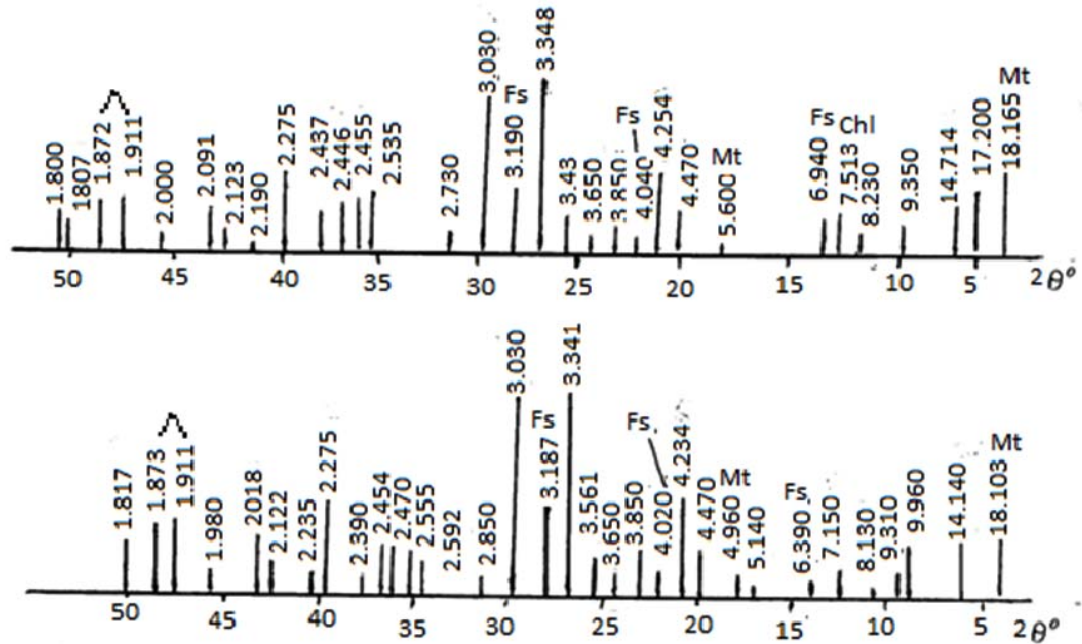

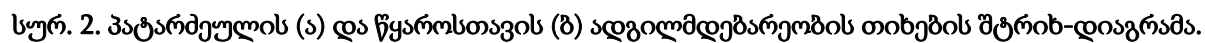

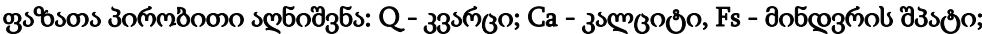

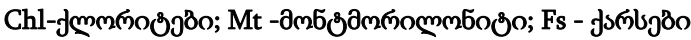




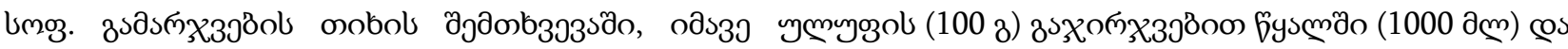

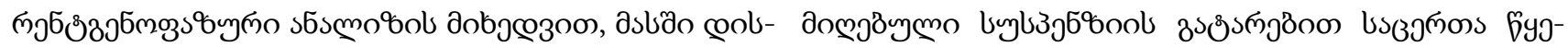

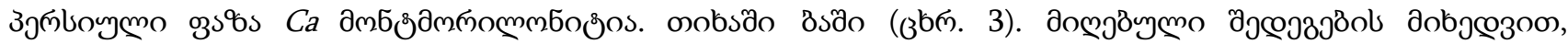

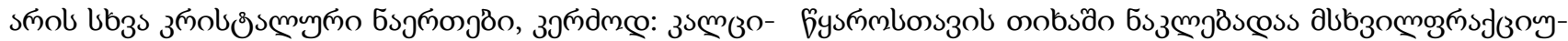

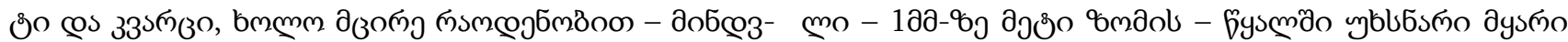

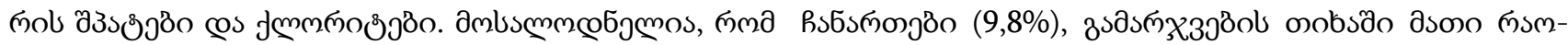

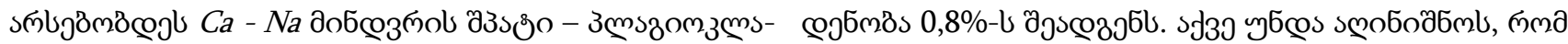

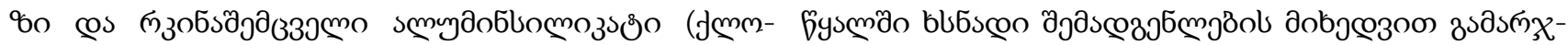

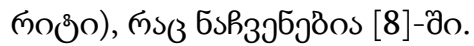

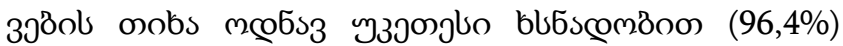

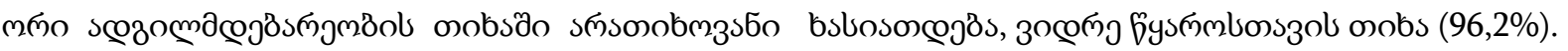

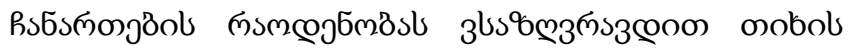

зbґомо 3

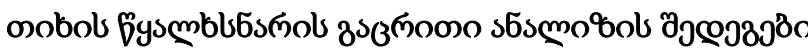

\begin{tabular}{|c|c|c|c|c|c|c|c|}
\hline \multirow{2}{*}{ 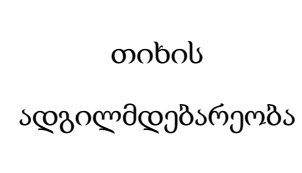 } & \multicolumn{6}{|c|}{ 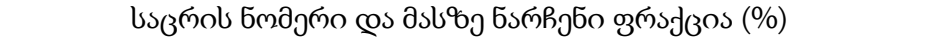 } & \multirow{2}{*}{$\begin{array}{c}x \mathrm{x} \partial \mathrm{o} \\
\%\end{array}$} \\
\hline & 10 & 5 & 2 & 1 & 0,5 & $\begin{array}{l}\text { astuymo 0,5 } \\
\text { изъэпдо }\end{array}$ & \\
\hline 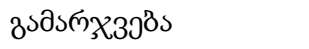 & 0,8 & 1,4 & 0,6 & 0,5 & 0,3 & 96,4 & 100,0 \\
\hline 6ysmmbonszo & $\mathrm{X}$ & $\mathrm{X}$ & 0,8 & 0,9 & 2,1 & 96,2 & 100,0 \\
\hline
\end{tabular}

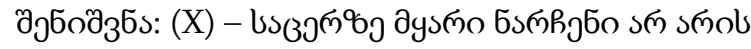

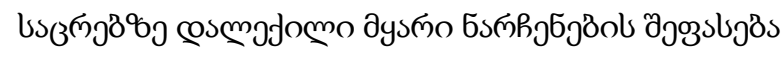

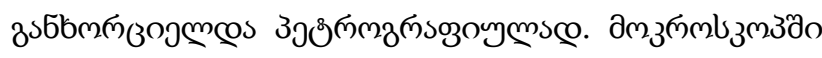

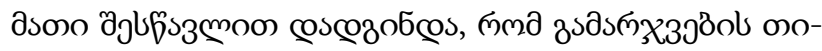

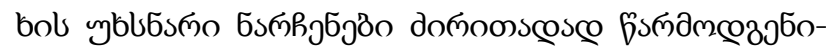

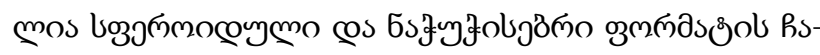

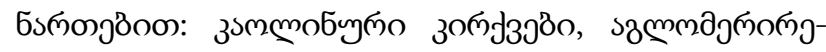

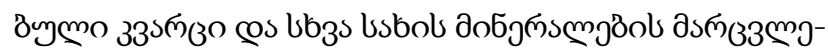

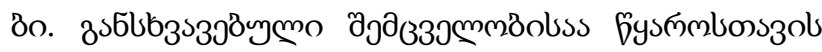

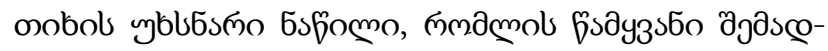
3эбృ

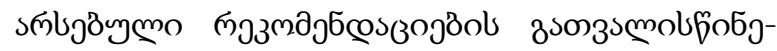

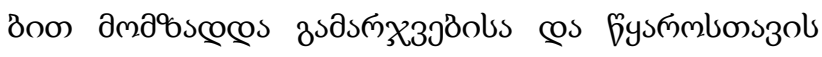

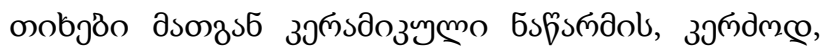

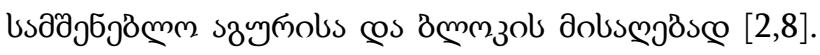
пззеззоп̆

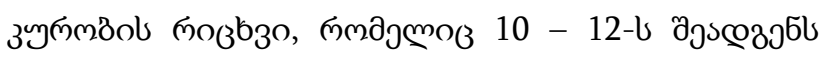

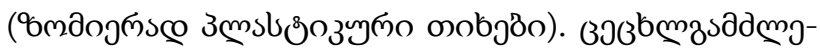

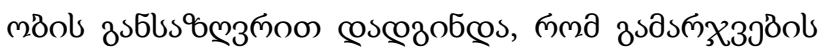

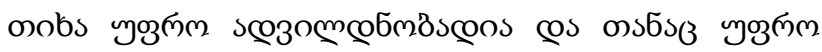

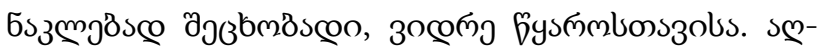

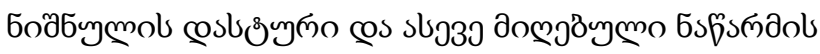

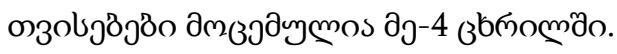


zsasm

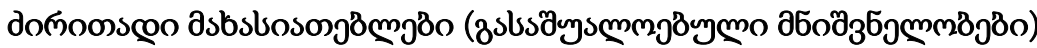

\begin{tabular}{|c|c|c|c|c|c|c|c|c|c|c|}
\hline \multirow[t]{2}{*}{$\begin{array}{c}\text { бody- } \\
\text { yol } \\
\text { N }\end{array}$} & \multicolumn{2}{|c|}{ 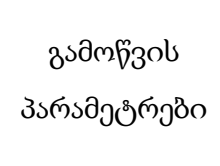 } & \multicolumn{2}{|c|}{$\begin{array}{c}\text { изупом } \\
\text { คзххщмдs, \% }\end{array}$} & \multicolumn{2}{|c|}{$\begin{array}{c}\text { 6ysm̃onsбonjas, } \\
\%\end{array}$} & \multicolumn{2}{|c|}{ 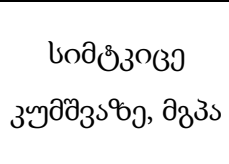 } & \multicolumn{2}{|c|}{ 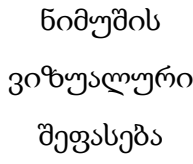 } \\
\hline & $\mathrm{T},{ }^{0} \mathrm{C}$ & $\tau_{\text {lom }}$ & A & B & A & B & A & B & A & B \\
\hline 1 & 850 & 1 & 5 & 10 & 40 & 14,9 & 3,6 & 13,8 & - & + \\
\hline 2 & 900 & 1 & 5 & 10 & 38 & 14,0 & 4,0 & 15,0 & - & - \\
\hline 3 & 950 & 1 & 5 & 11 & 32 & 13,5 & 4,6 & 15,1 & - & - \\
\hline 4 & 1000 & 1 & 6 & 12 & 31 & 13,0 & 5,4 & 15,4 & - & + \\
\hline 5 & 1050 & 1 & 6 & 12 & 29 & 12,7 & 6,5 & 15,8 & - & + \\
\hline
\end{tabular}

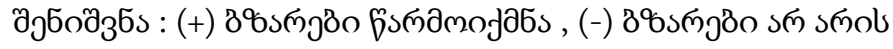

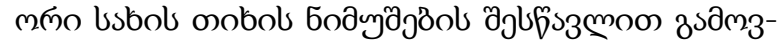
moбẹs ol osszobjäyng

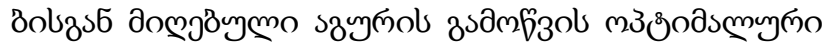

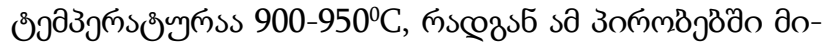

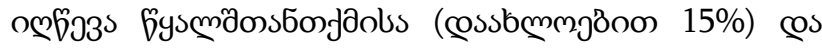

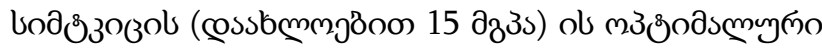

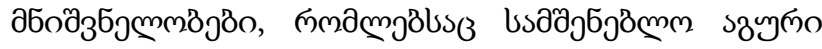

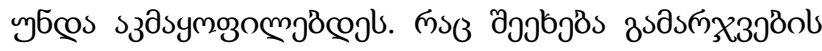

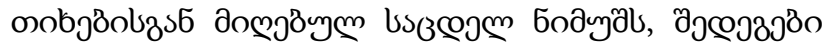

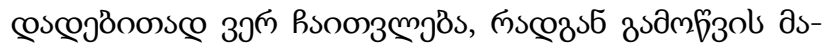

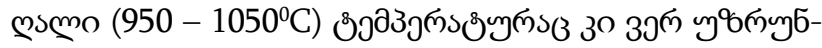

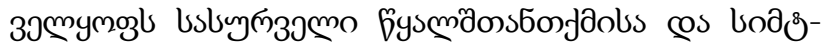

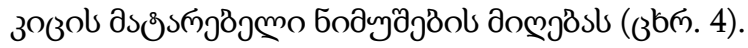

\section{$\cos 333^{6 s}$}

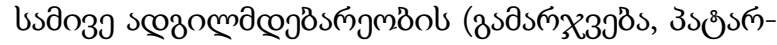

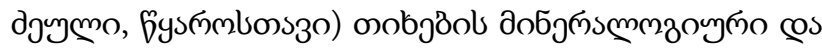

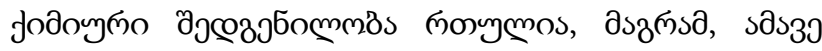

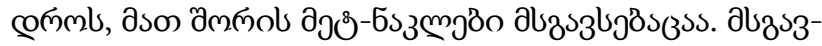

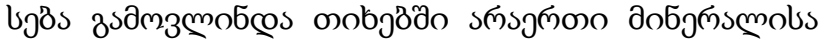

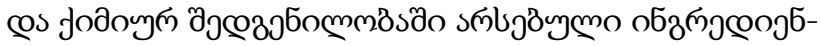

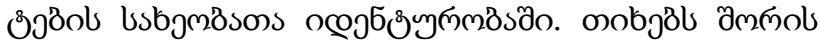

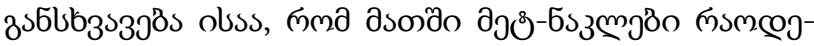

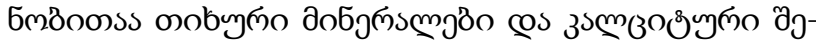

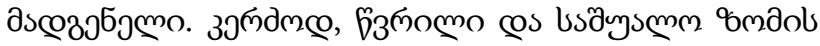

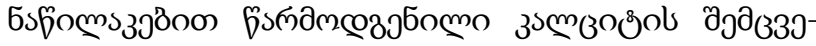

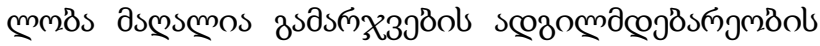

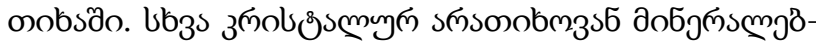

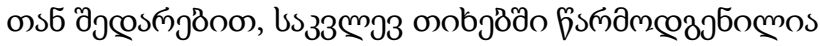

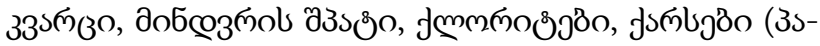

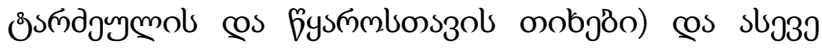

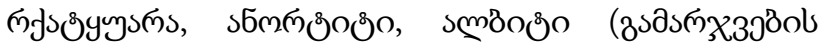

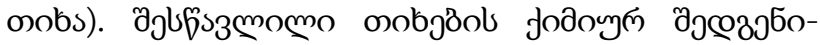

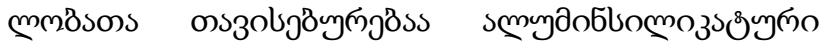
$\left(\mathrm{SiO}_{2}+\mathrm{Al}_{2} \mathrm{O}_{3}\right)$ cos mzobols mfluocoos $\left(\mathrm{FeO}+\mathrm{Fe}_{2} \mathrm{O}_{3}\right)$

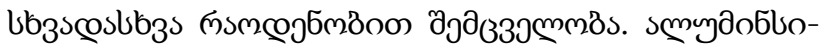

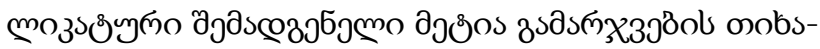

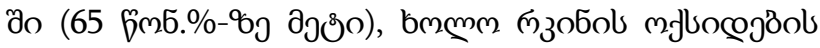




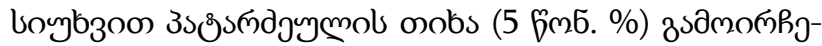

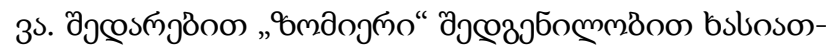

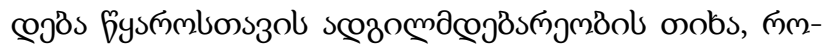

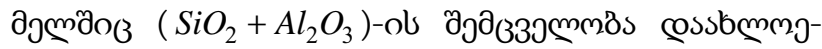

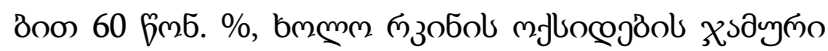

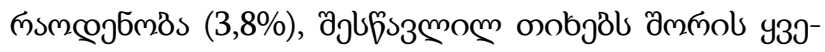

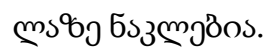

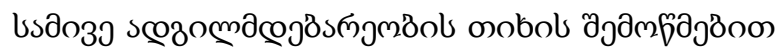

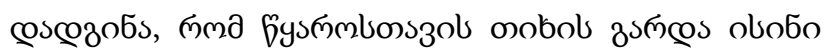

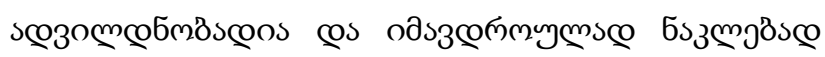

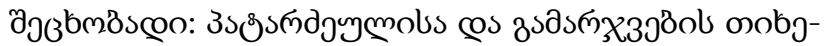

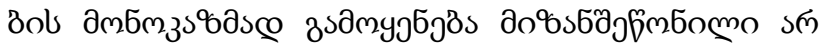

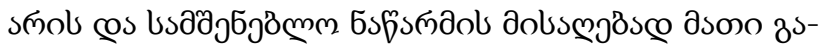

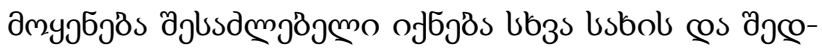

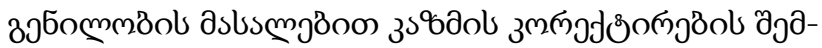

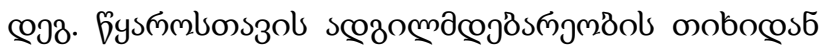

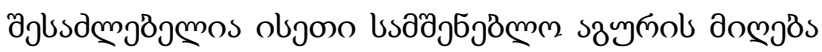

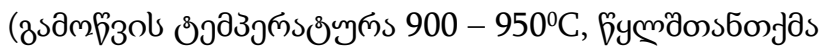

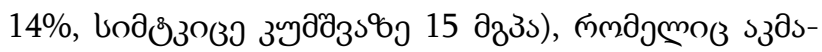

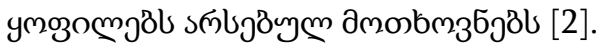

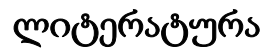

1. URL: https://znaytovar.ru/s/Klassifikaciya_i_assortiment_ke.html (in Russian).

2. Budnikov P.P. The technology of ceramics and refractories. The MIT Press. 1964, 374 p. (in Russian).

3. URL: https://studopedia.org/6-45351.html (in Russian).

4. GOST 9169-75. Raw materials for ceramics industry. (in Russian).

5. URL: http://www.mining-enc.ru/g/gliny/ (in Russian).

6. URL: https://dic.academic.ru/dic.nsf/enc_geolog/1377/\%D0\%93\%D0\%B8\%D0\%B4\%D1\%80\%D0\%BE\%D1\% 81\%D0\%BB\%D1\%8E\%D0\%B4\%D1\%8B (in Russian).

7. URL: http://www.mining-enc.ru/g/gidroslyudy/ (in Russian).

8. Augustinik A. I. Ceramics. L.: "Stroyizdat". 1975, 590 p. (in Russian).

9. Natural resources of the Georgian USSR. Non-material useful minearls. AN USSR. Vol. II. M. 1959. (in Russian). 


\title{
UDC 666.953
}

SCOPUS CODE 1508

DOI: https://doi.org/10.36073/1512-0996-2019-3-19-28

\section{Study of chemical-mineralogical composition and possibilities of practical application of clays from three locations of Kartli-Kakheti}

\author{
Givi Loladze Department of Chemical Technology and Biotechnology, Georgian Technical \\ University, 69 M. Kostava str, 0160 Tbilisi, Georgia \\ E-mail: g.loladze@gtu.ge \\ Teimuraz Cheishvili Department of Chemical Technology and Biotechnology, Georgian Technical \\ University, 69 M. Kostava str, 0160 Tbilisi, Georgiaa \\ E-mail: t.cheishvili@gtu.ge
}

\section{Reviewers:}

G. Tabatadze, Associate Professor, Faculty of Chemical Technology and Metallurgy, GTU

E-mail: g.tabatadze@yahoo.com

V. Gordeladze, Associate Professor, Faculty of Chemical Technology and Metallurgy, GTU

E-mail: b.gordeladze@gtu.ge

Abstract. The main objective of the research was to establish mineralogical and chemical composition of the argillaceous rocks from Gamarjveba, Patardzeuli and Tskarostavi locations, to assess the possibilities of manufacturing the building materials (brick, tiles) from them and to identify appropriate technological parameters.

Establishment of mineralogical and chemical composition of examined clays through petrographic methods, Xray phase analysis and complete chemical analysis of silicates as well as article manufacture from clays and study of their basic and peculiar properties had been conducted.

Mineralogical and chemical composition of the clays from three locations was identified and their comparative study was conducted. Molding ability of clays was determined, test samples were molded and baked and their properties were studied as well.

Examined clays are characterized by complex and different mineralogical and chemical composition at the same time. Clays are distinguished by plasticity index acceptable for enterprises and high water solubility. For obtaining the ceramic items for industrial purposes it is recommended to use the clays from Tskarostavi location, which provide receipt of items with desirable operational properties at $900-950^{\circ} \mathrm{C}$.

Key words: Baking; clay; item; mineralogy; molding; phase composition; properties. 
UDC 666.953

SCOPUS CODE 1508

DOI: HTTPS://DOI.ORG/10.36073/1512-0996-2019-3-19-28

\section{Изучение химико-минералогического содержания и возможности практического использования глин находящихся на трёх картлийско-кахетинских территориях}

Гиви Лоладзе

Теимураз Чеишвили
Департамент химической и биологической технологий, Грузинский технический университет, Грузия, 0160, Тбилиси, ул. М. Костава, 69

E-mail: g.loladze@gtu.ge

Департамент химической и биологической технологий, Грузинский технический университет, Грузия, 0160, Тбилиси, ул. М. Костава, 69

E-mail: : t.cheishvili@gtu.ge

\section{Рецензенты:}

Г. Табатадзе, кандидат технических наук, ассоциированный профессор факультета химической технологии и металлургии ГТУ

E-mail: g.tabatadze@yahoo.com

В. Горделадзе, кандидат технических наук, ассоциированный профессор факультета химической технологии и металлургии ГТУ

E-mail: b.gordeladze@gtu.ge

Аннотация. Изучение месторождения глинистых пород селений Гамарджвеба, Патардзеули и Цкаростави - установление минералогического и химического состава и оценка возможности получения из них материала (кирпича, плиток) для строительного назначения, установления технологических параметров петрографическими методами, полным рентгенофазовым и силикатным химическим анализом, важно для изучения их практического использования.

Установлен химико-минералогический состав глин трёх месторождений. Определена способность формовки - обжига глин, осуществилась калибровка-обжиг исследуемого образца, после изучения были сравнены их качества. Исследованные глины характеризуются сложным и в то же время разным минералогическим и химическим составом, приемлемым для производства числом пластичности и хорошим растворением в воде. Для получения керамического продукта строительного назначения рекомендуется применение глины Цкароставского участка, которая обеспечивает получение продукта желательных эксплуатационных свойств на $900-950^{\circ} \mathrm{C}$.

Ключевые слова: выжигание; глина; качество; минералогия; продукт; фазовое содержание; формовка.

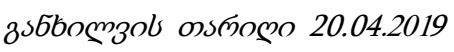

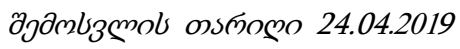

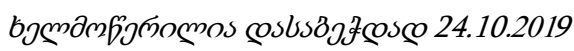




\section{UDC 004.942}

\section{SCOPUS CODE 1706}

DOI: https://doi.org/10.36073/1512-0996-2019-3-29-39

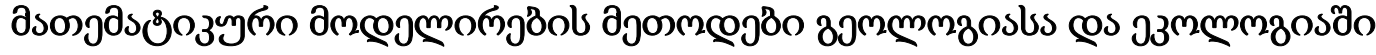

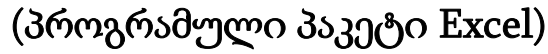

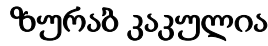

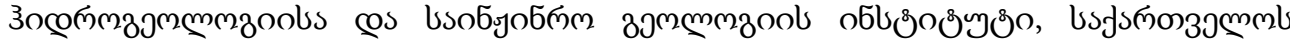

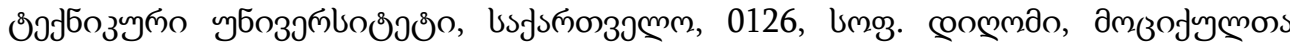

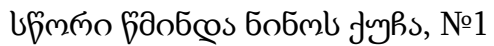

E-mail: zukakulia@yahoo.com

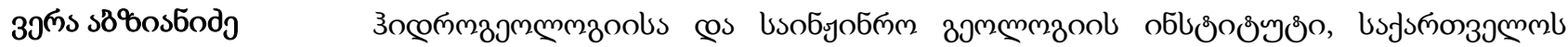

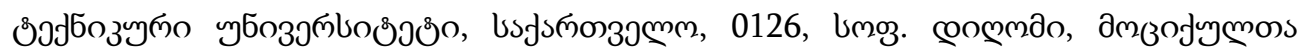

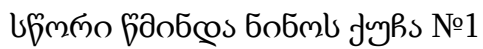

E-mail: veriko_abz@mail.ru

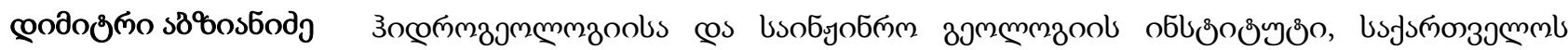

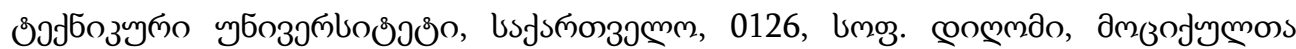

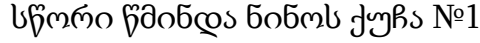

E-mail: dimitri.abz@mail.ru

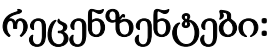

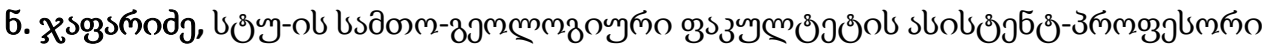

E-mail: ninojap@mail.ru

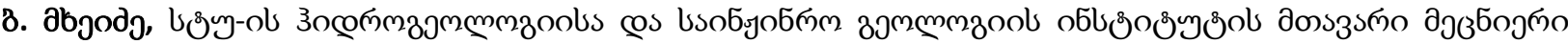

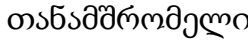

E-mail: b.mkheidze@gtu.ge

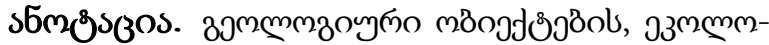

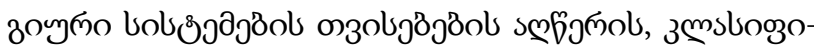

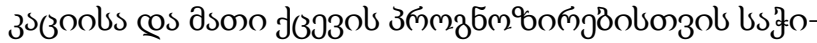

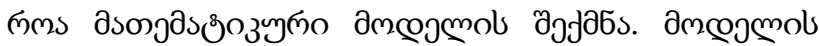

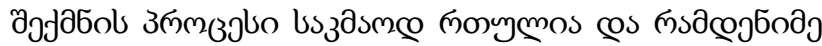

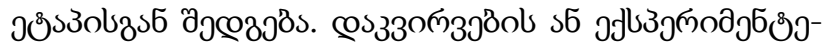

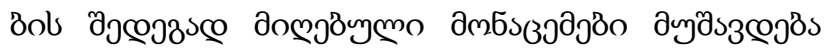

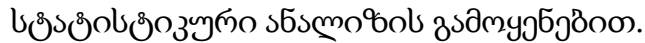

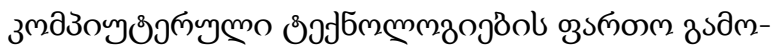

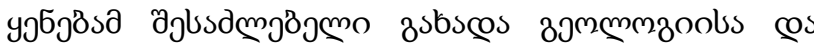

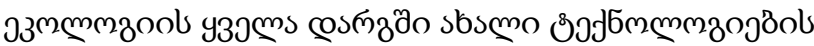

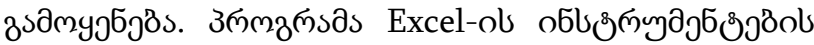

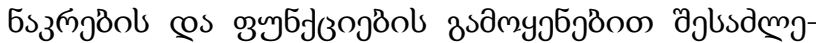

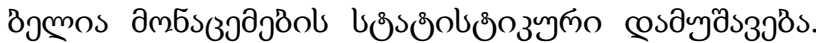

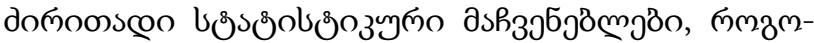

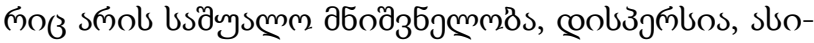

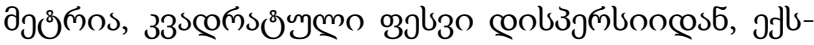

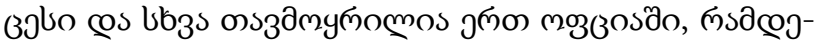




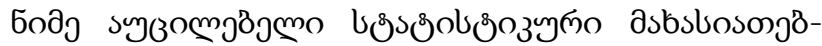

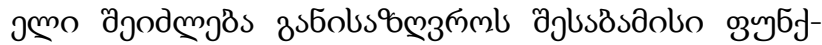

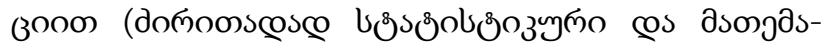

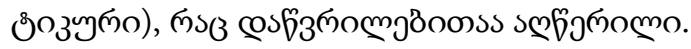

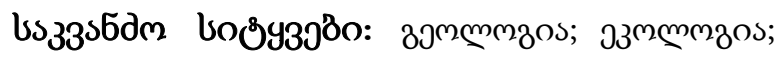

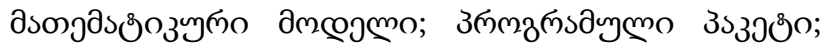

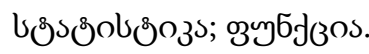

\section{gjuszsmo}

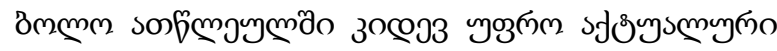

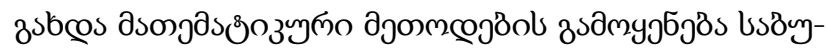

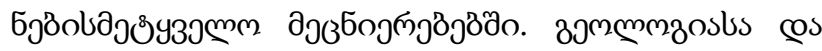

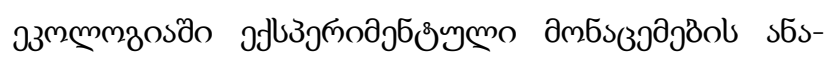

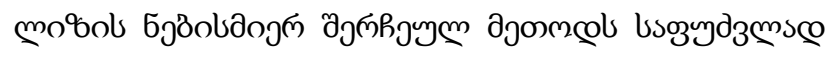

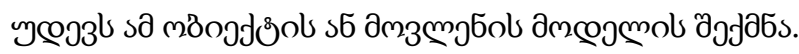

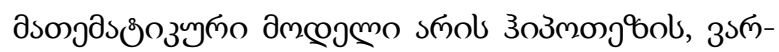

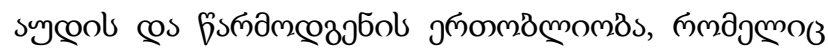

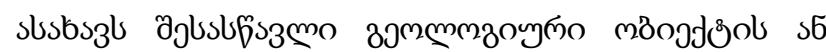

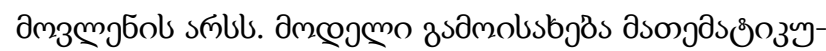

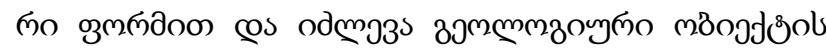

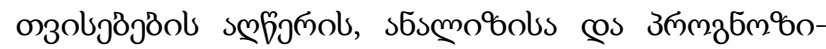

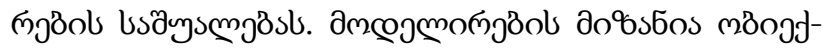

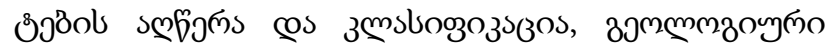

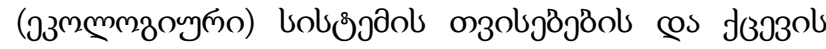

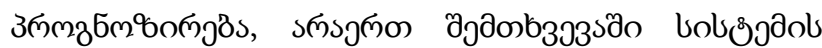

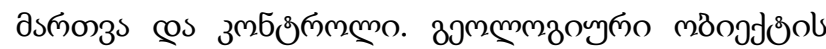

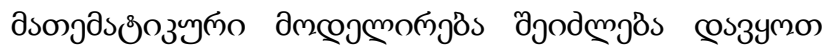

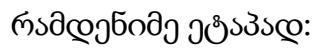

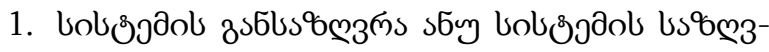
mjôol coscajбs;

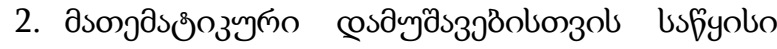

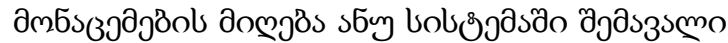
mరింวปฮீ

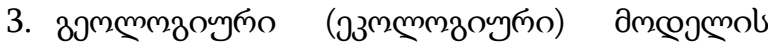

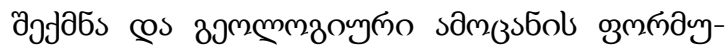
monjos;

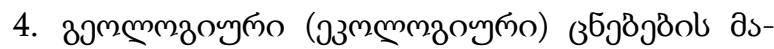

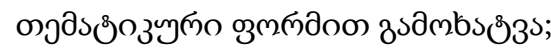

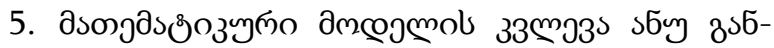

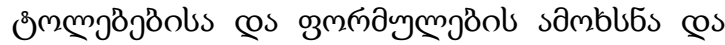

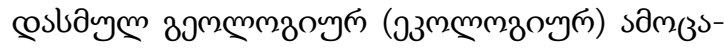
6s\%g 3sluybol domg8s;

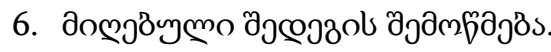

\section{domoosseo 6sformo}

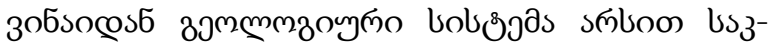

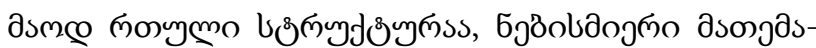

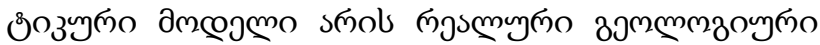

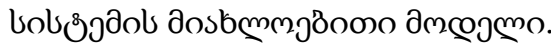

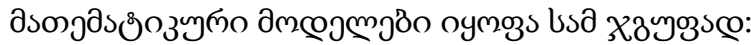

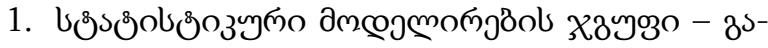

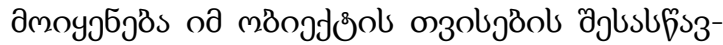

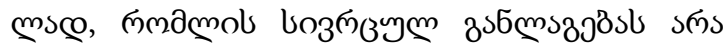

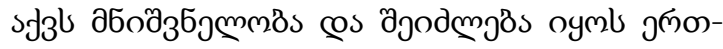
зsб\%мдам

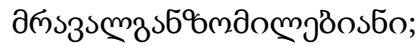

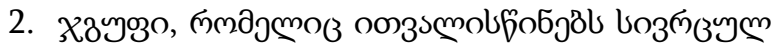

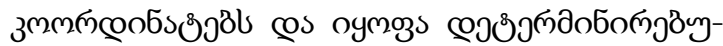

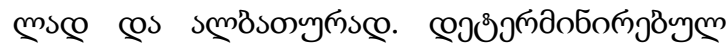

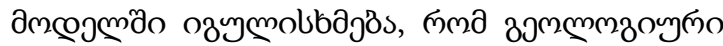

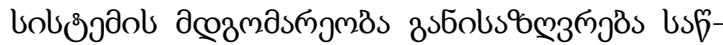

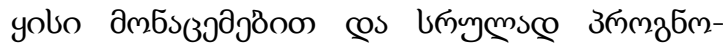

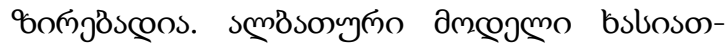

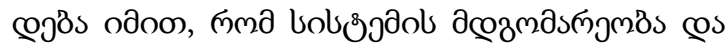




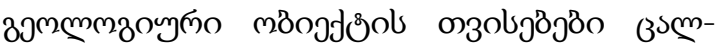

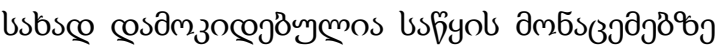

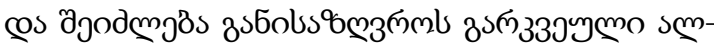

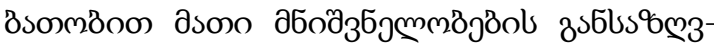

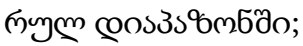

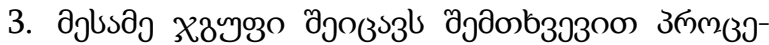

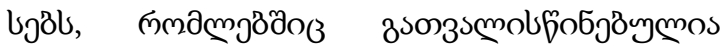

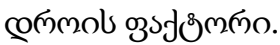

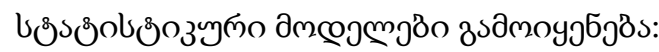

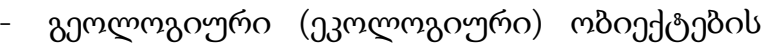
змmsluozozszoolsonzol;

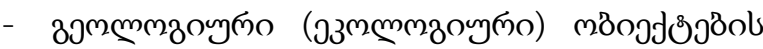

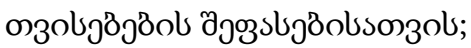

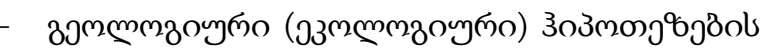

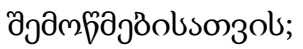

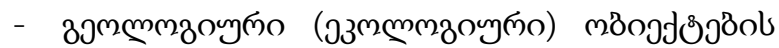

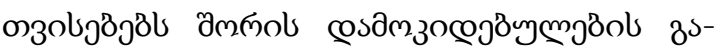

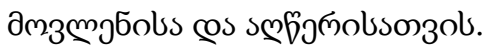

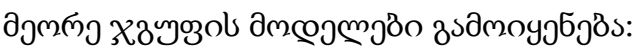

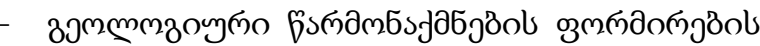

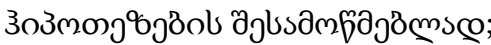

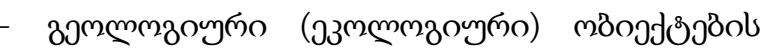

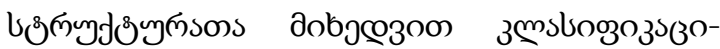
obo3ol;

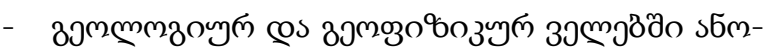
asmogßob zsamymogobsonzob;

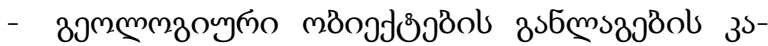

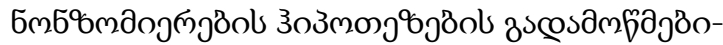
uson3ol.

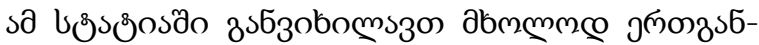

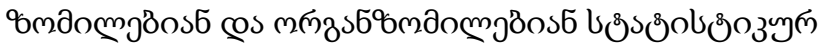

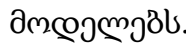

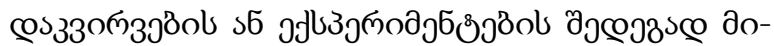

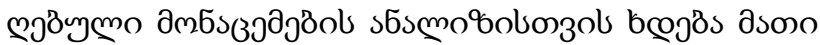

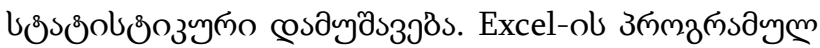

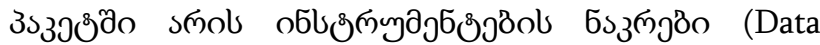

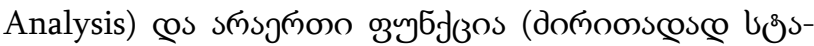

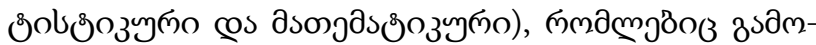

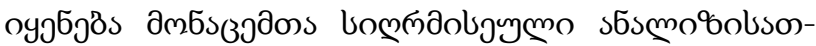

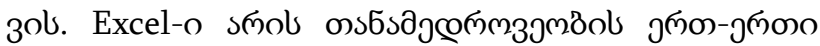

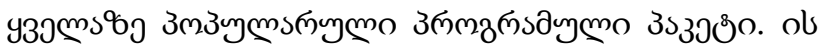

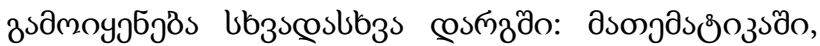

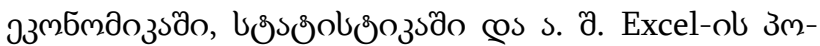

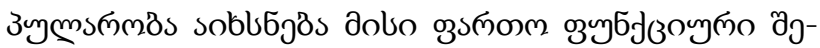

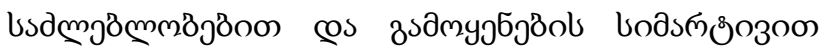

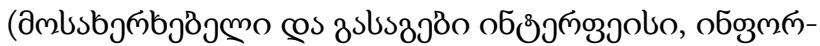

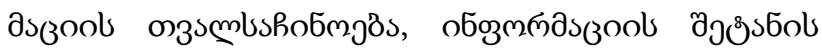
uobfrnsog cos s.ð.).

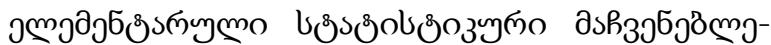
Boblonzol ajodmgäs zsamzoygбmon Data Analysis-ols mo3̧os Descriptive Statistics. dobo doøিonsco dsbs-

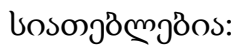

\begin{tabular}{|c|}
\hline 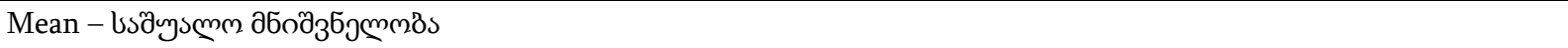 \\
\hline 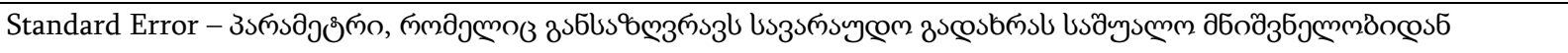 \\
\hline 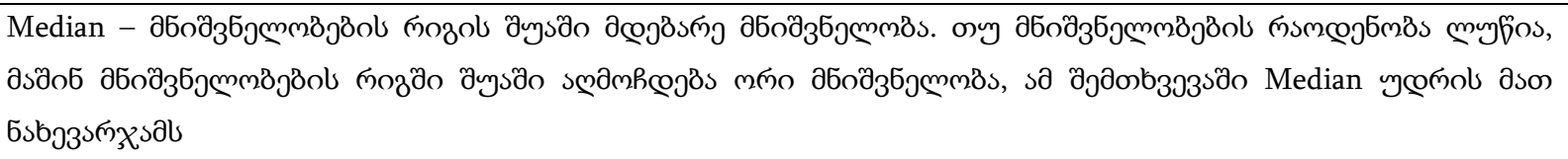 \\
\hline
\end{tabular}




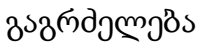

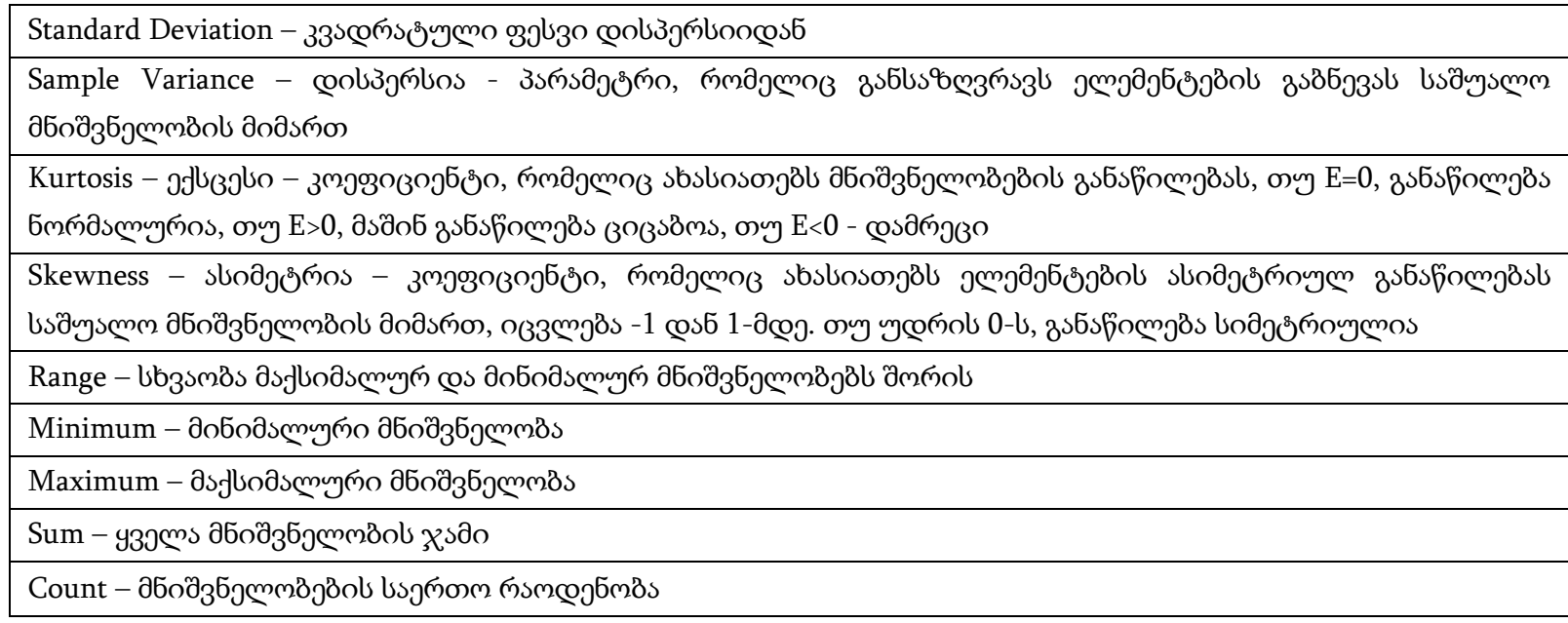

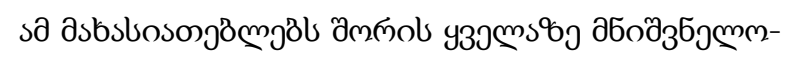
3s5os Mean, Standard Error eos Standard Deviation. Standard Deviation @os Sample Variance sbsbs 395

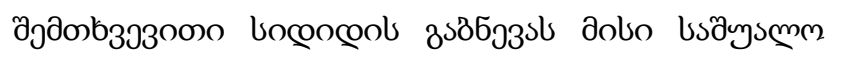

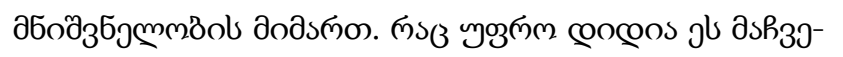

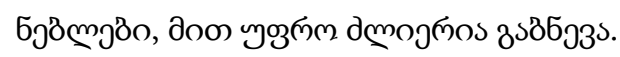

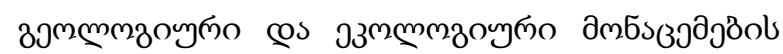

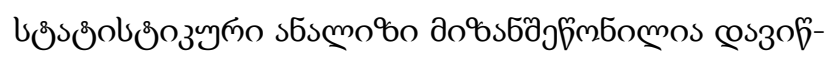

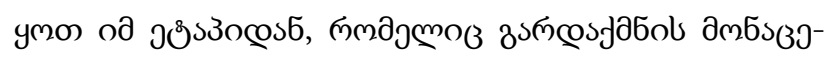

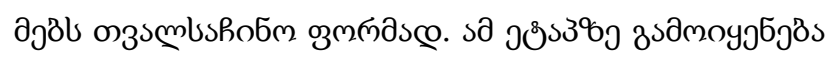

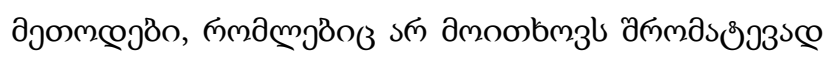

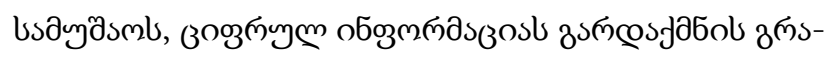

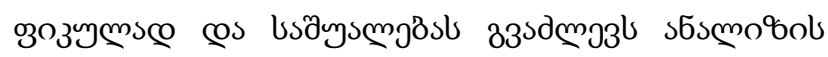

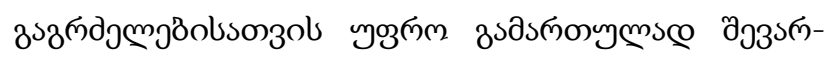

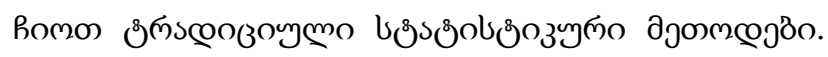

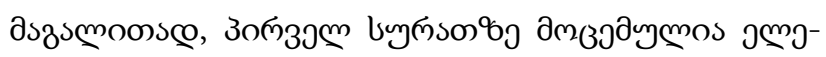

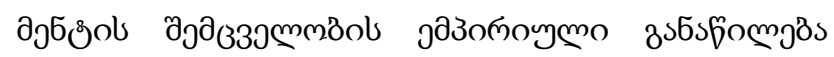

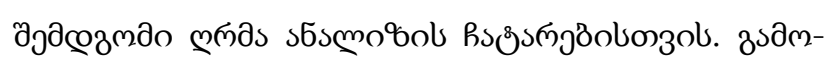

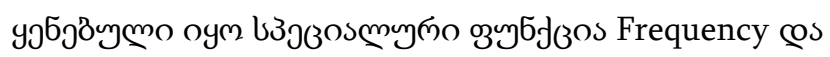

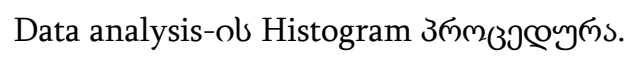

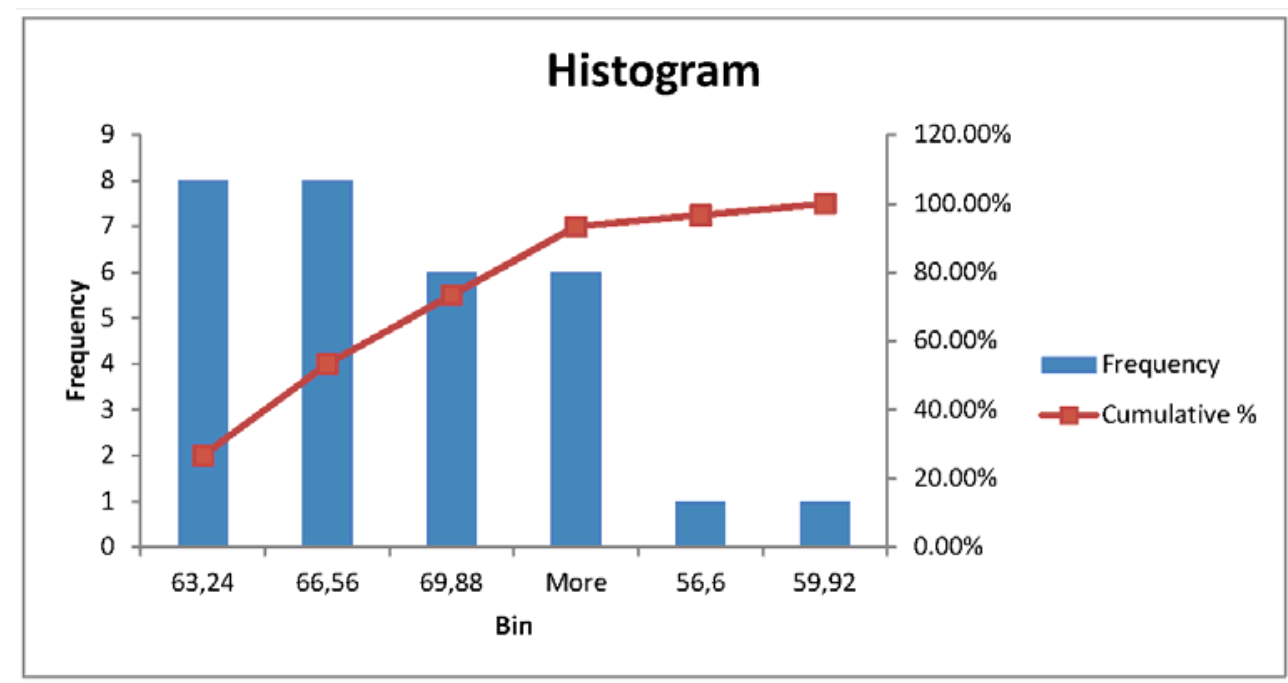

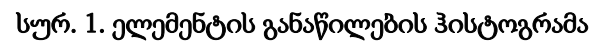




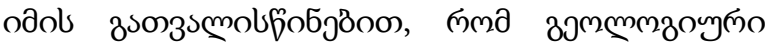

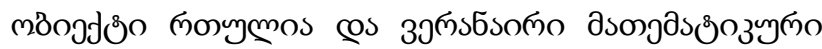

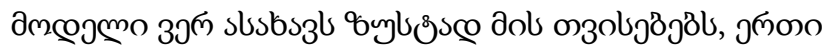

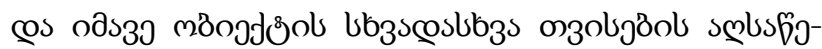

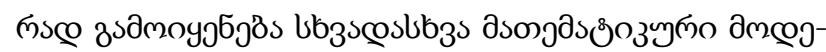

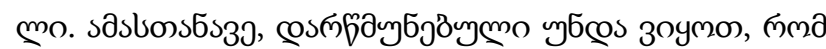

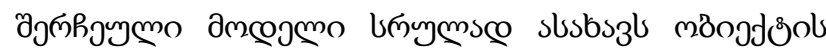

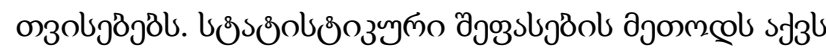

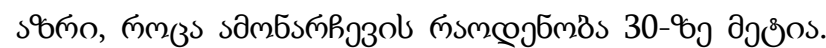

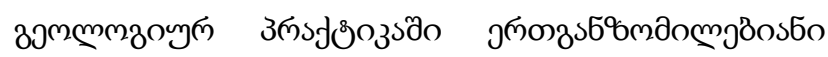

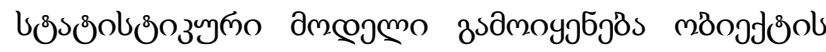

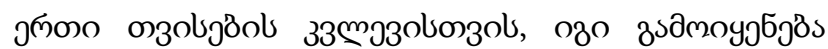

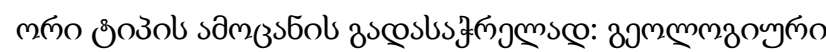

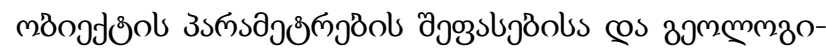

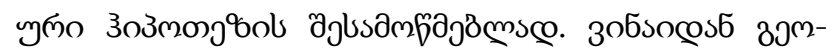

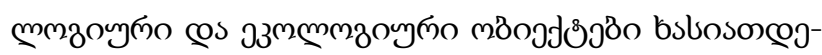

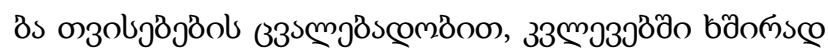

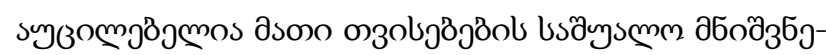

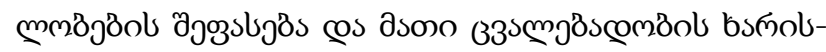

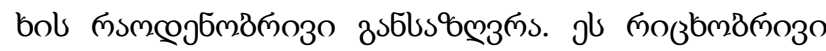

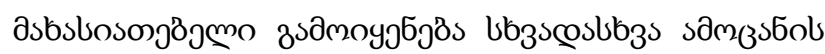

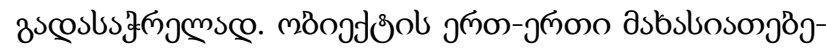

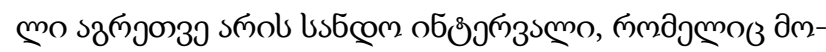

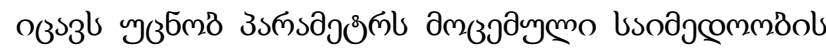

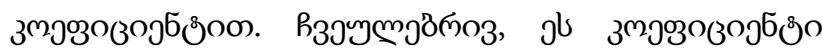

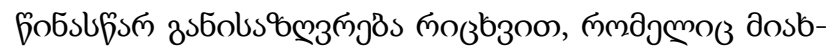

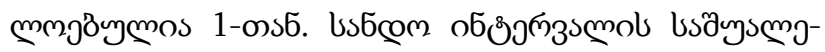

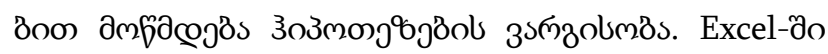
usбçm oб

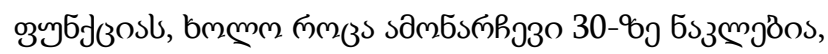

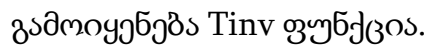

usбçm oб

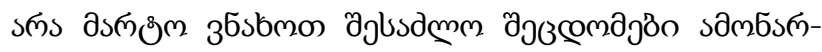

\section{ISSN 1512-0996}

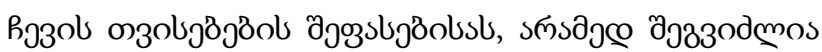

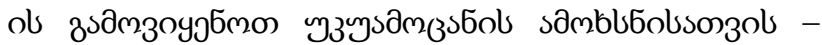

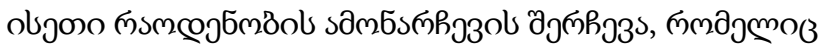

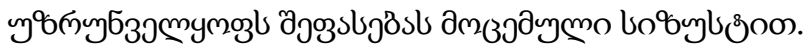

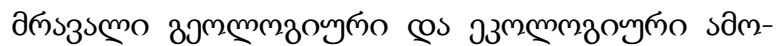

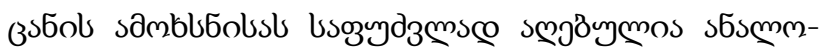

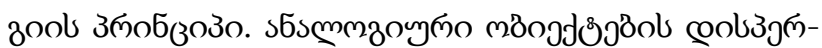

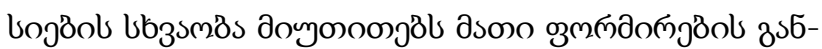

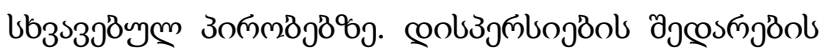

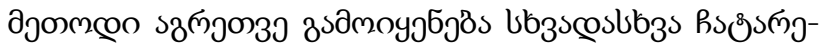

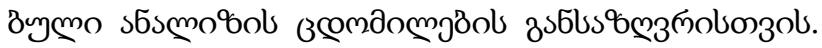

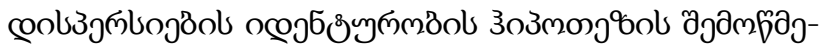

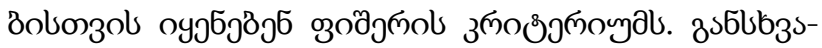

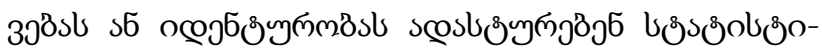

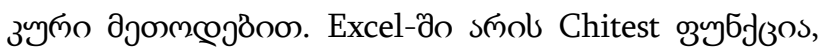

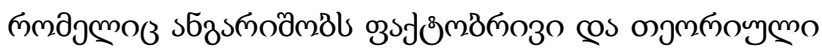

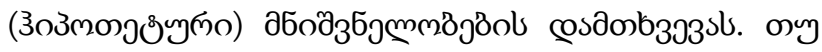

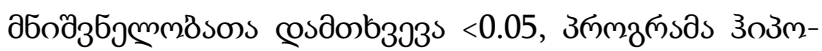

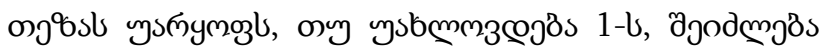

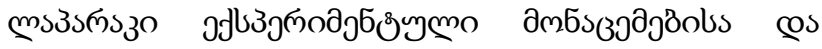

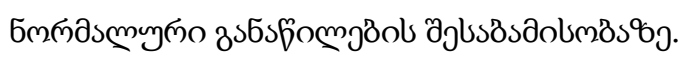

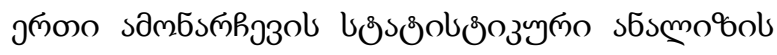

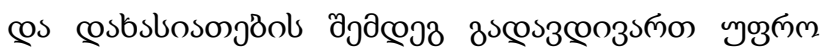

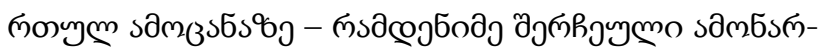

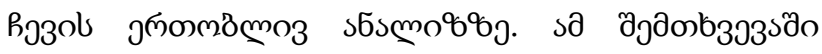

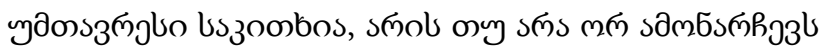

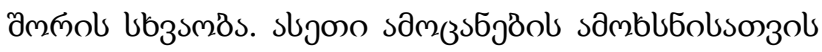

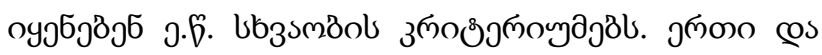

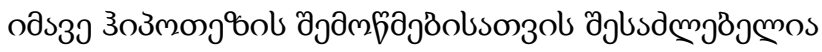

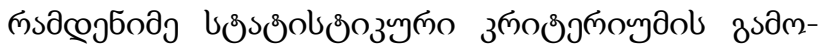

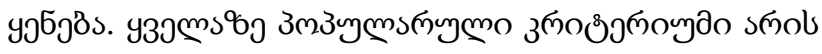

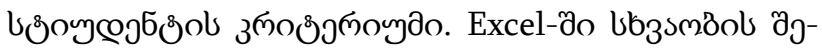

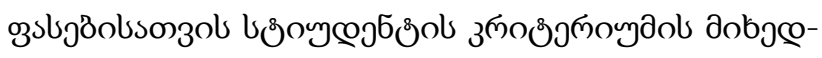




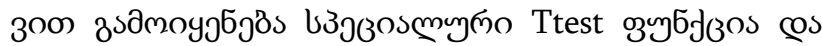

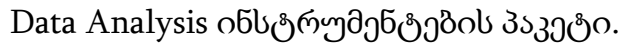

мко sдмбsк̆

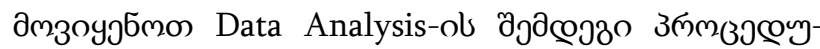
๓ூס̊ం: t-Test Paired Two Sample for Means, t-Test Two Sample Assuming Equal Variances, t-Test Two Assuming Unequal Variances.

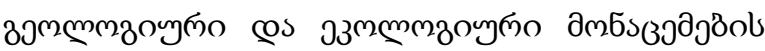

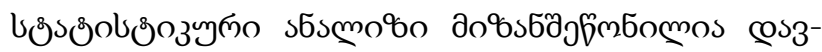

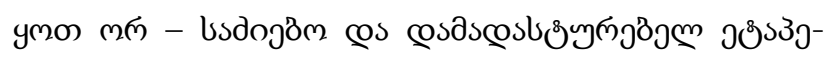

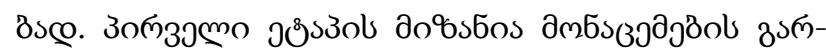

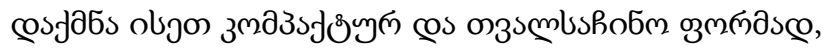

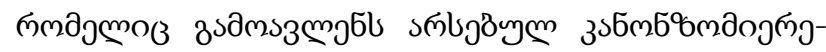

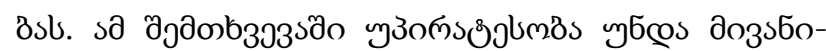

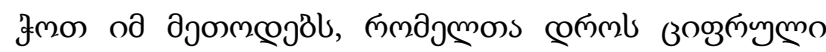

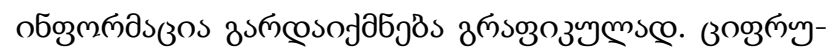

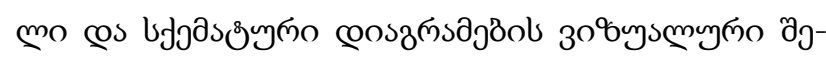

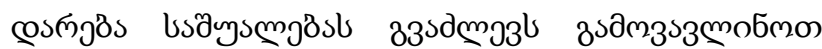

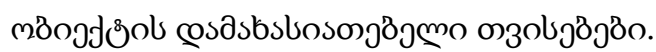

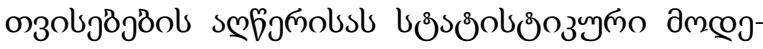

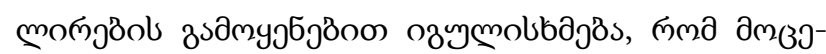

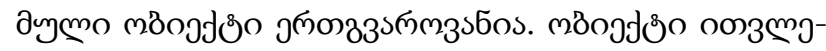

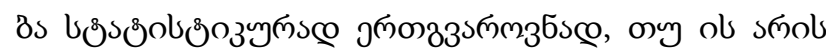

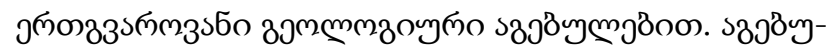

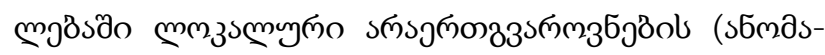

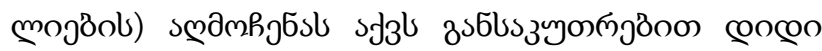

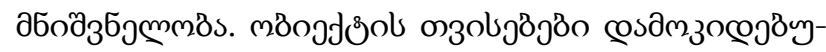

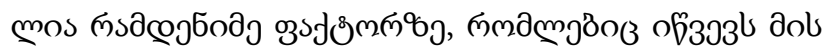

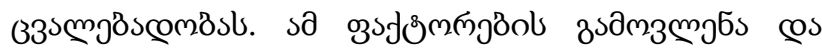

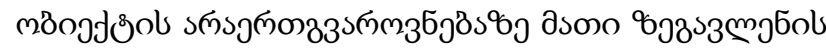

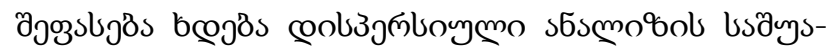

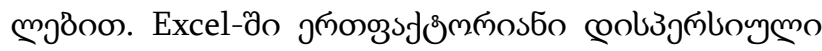

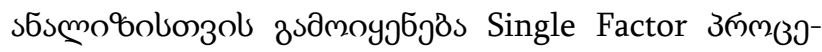

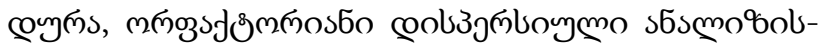
o3ol-Two-Factor Replication @s Two-Factor without

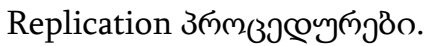

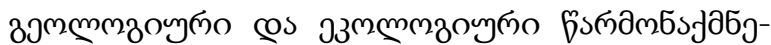

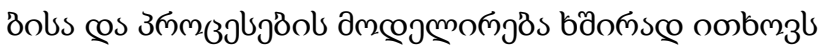

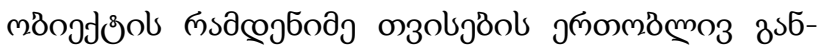

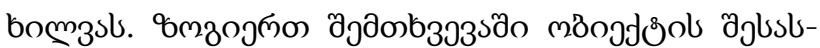

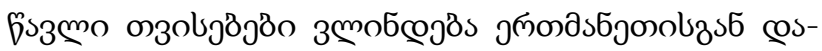

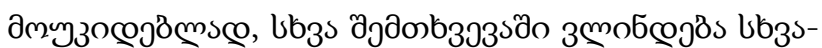

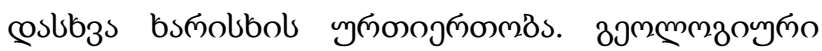

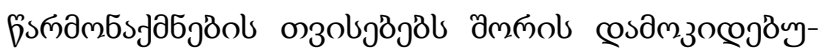

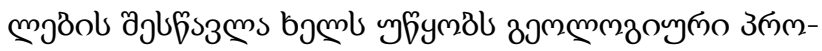

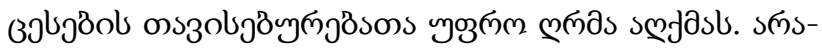

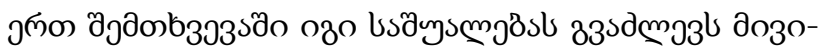

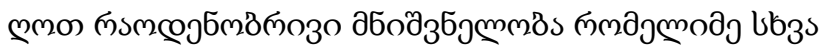

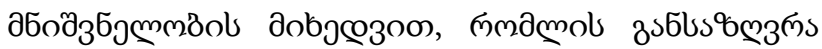

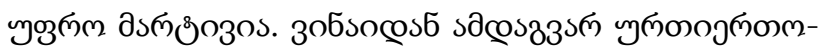

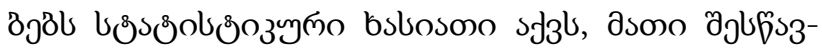

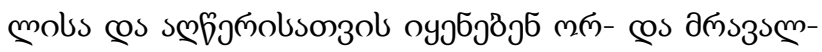

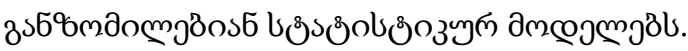

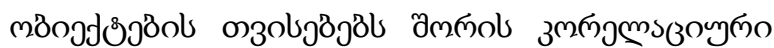

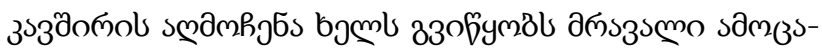

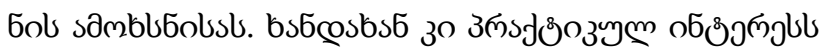

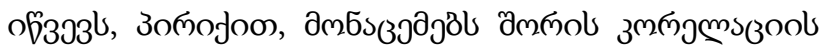

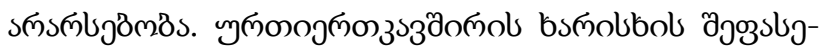

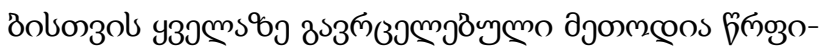

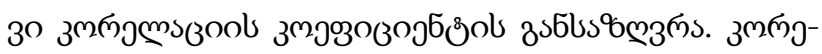

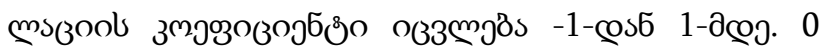

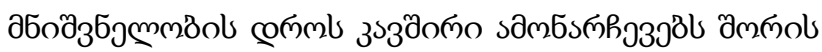

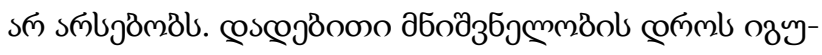

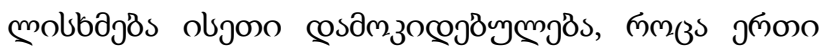

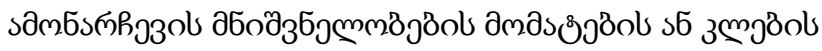

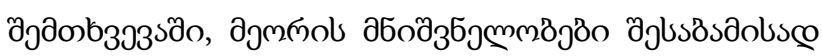




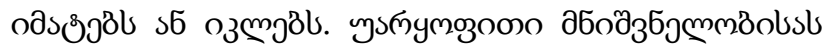

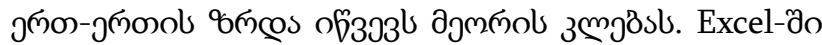

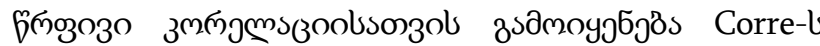

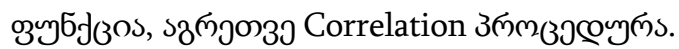

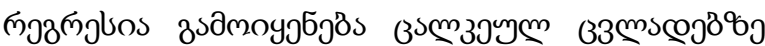

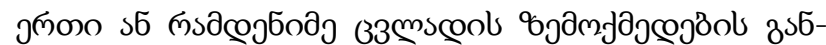

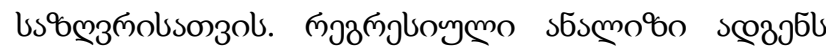

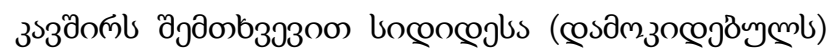

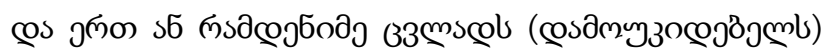

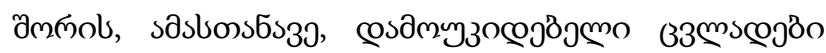

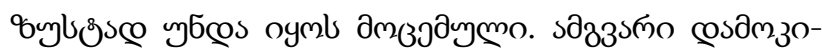

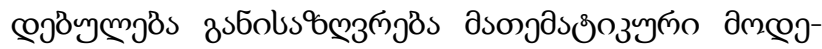

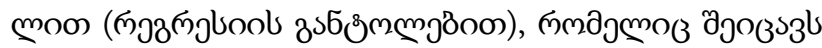

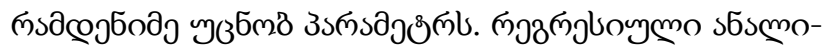

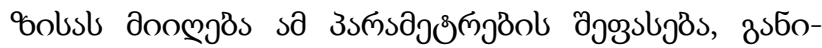

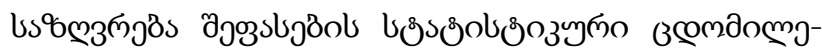

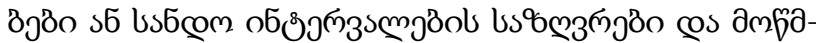

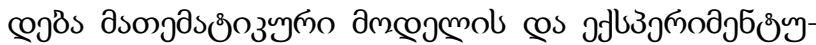

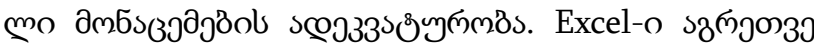

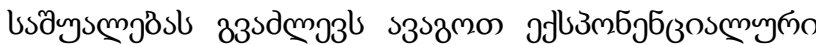
mozmglos. saobsonzols smbgambl Growth cos Logest

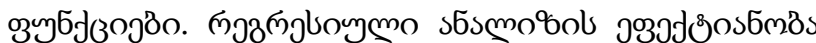

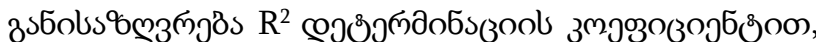

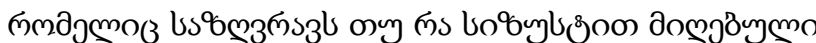

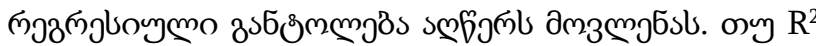

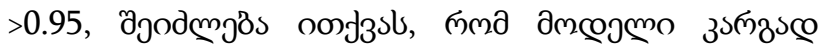

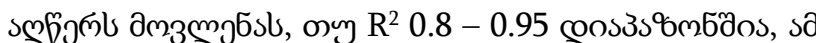

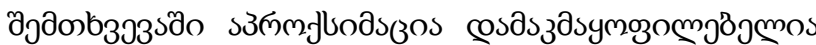

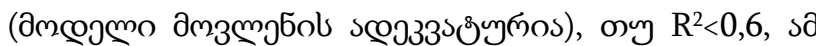

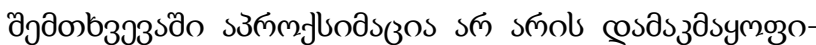

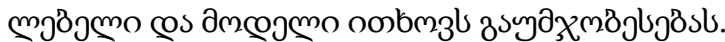

\begin{tabular}{lr}
\hline \multicolumn{2}{c}{ Regression Statistics } \\
\hline Multiple R & 0,978578674 \\
R Square & 0,957616221 \\
Adjusted R Square & 0,955123058 \\
Standard Error & 15,66506144 \\
Observations & 19 \\
\hline
\end{tabular}

ANOVA

\begin{tabular}{|c|c|c|c|c|c|}
\hline & $d f$ & $S S$ & $M S$ & $F$ & Significance $F$ \\
\hline Regressio & 1 & 94255,11971 & 94255,11971 & 384,0968488 & $4,168 \mathrm{E}-13$ \\
\hline Residual & 17 & 4171,700549 & 245,3941499 & & \\
\hline Total & 18 & 98426,82026 & & & \\
\hline
\end{tabular}

\begin{tabular}{|c|c|c|c|c|c|c|}
\hline & & Coefficients & Standard Error & t Stat & $P$-value & Lower 95\% \\
\hline Intercept & & $-15,38108394$ & 10,41818423 & $-1,47636897$ & 0,158128556 & $-37,3615313$ \\
\hline & 15,906 & 4,314836586 & 0,220162821 & 19,59838893 & $4,168 \mathrm{E}-13$ & 3,850333637 \\
\hline
\end{tabular}




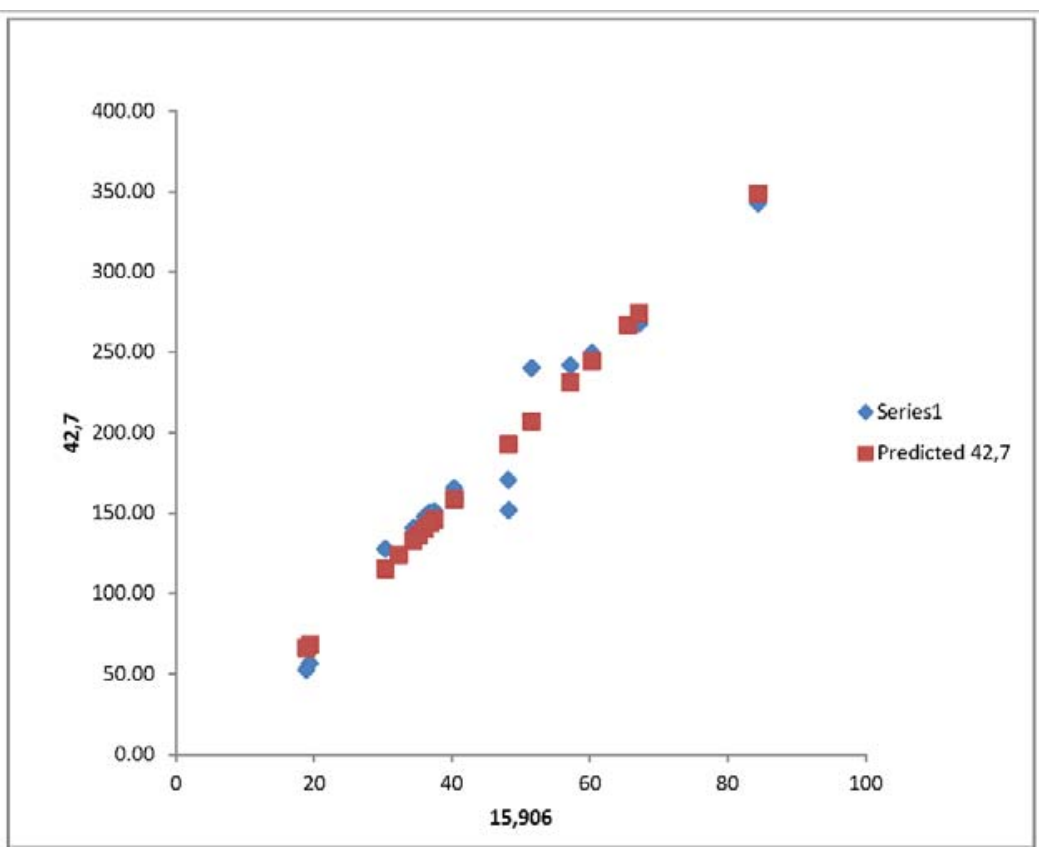

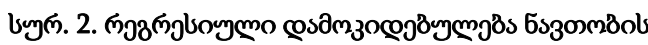

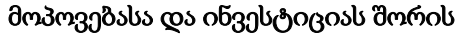

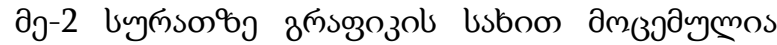

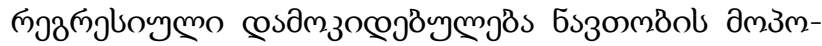

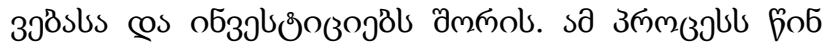

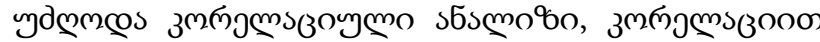

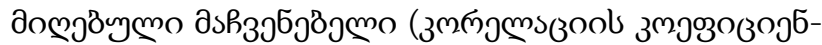

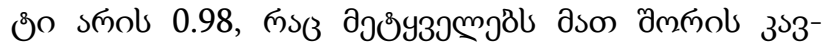

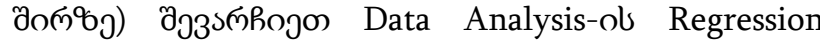

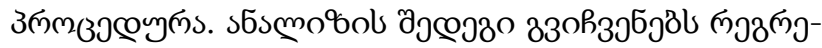

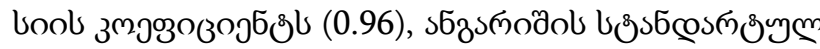

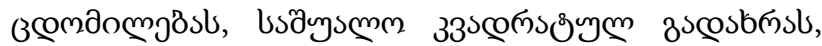

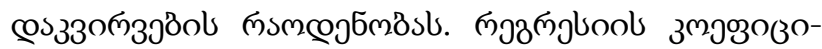

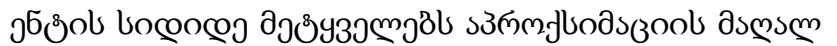

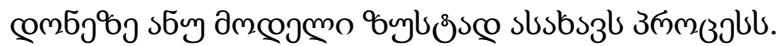

\section{cosb336s}

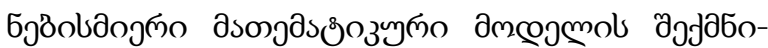

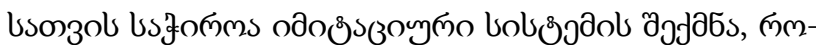

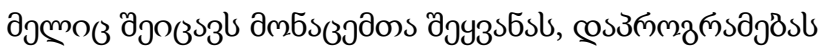

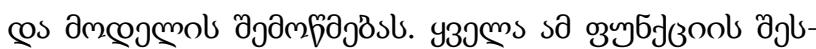

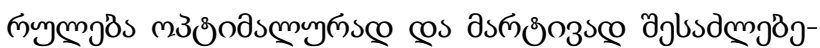

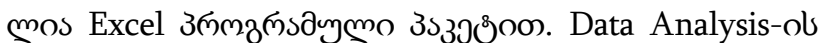

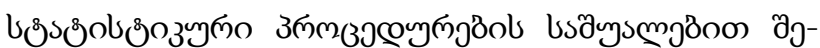

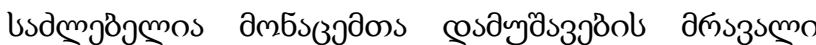

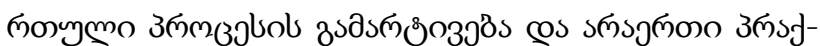

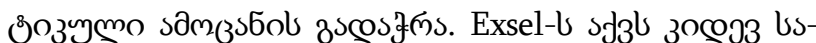

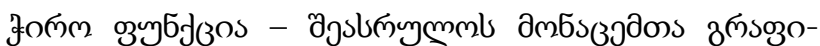
зулмо sбsmo\%o. 


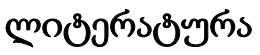

1. Walkenbach J. Excel 10 Bible. Wiley; 1 edition. 2010.

2. Diakonov V.V., Gorg N.V. Computer methods for processing geological information. Moscow. 2008. (in Russian).

3. Korbunov A.I. Mathematical modeling methods in applied geophysics. Part I. Functional and analytical framework. Moscow. 2014. (in Russian).

4. Belkina V.A., Bembel S.R., Zaboeva A.A., Sankova N.V. Fundamentals of geological modeling. Tyumen: Tyumen State Technical University. 2015. (in Russian).

5. Carlberg C. Predictive analytics: Microsoft Excel. Colorado. 2012.

6. Alexander M., Kusleika R. Excel 2016 Formulas. 2017.

7. Shaorshadze M. Mathematical statistics in geology. Tbilisi. 1980. (in Georgian).

8. Abzianidze D., Tabatadze G., Khundadze N., Meskhishvili T. Use of mathematical modeling methods to solve environmental tasks. Tbilisi. 2013. (in Georgian).

9. Pantsulaia G., Kvatadze Z., Giorgadze G. Probability theory and mathematical statistics. Part I. Tbilisi. 2007. (in Georgian).

10. Pantsulaia G., Kvatadze Z., Giorgadze G. Probability theory and mathematical statistics. Part II. Tbilisi. 2007. (in Georgian). 


\title{
UDC 004.942
}

SCOPUS CODE 1706

DOI: https://doi.org/10.36073/1512-0996-2019-3-29-39

\section{Mathematical model methods in geology and ecology (Excel Software)}

\author{
Zurab Kakulia Institute of Hydrogeology and Engineering Geology, 1 Tsminda Nino str, Digomi \\ Village, 0126 Tbilisi, Georgia \\ E-mail: zukakulia@yahoo.com \\ Vera Abzianidze Institute of Hydrogeology and Engineering Geology, 1 Tsminda Nino str, Digomi \\ Village, 0126 Tbilisi, Georgia \\ E-mail: veriko_abz@mail.ru \\ Dimitri Abzianidze Institute of Hydrogeology and Engineering Geology, 1 Tsminda Nino str, Digomi \\ Village, 0126 Tbilisi, Georgia \\ E-mail: dimitri.abz@mail.ru
}

\section{Reviewers:}

N. Japaridze, Assistant Professor, Faculty of Mining and Geology, GTU

E-mail: ninojap@mail.ru

B. Mhkheidze, Chief Research Scientist, Institute of Hydrogeology and Engineering Geology

E-mail: b.mkheidze@gtu.ge

Abstract. For the description and classification of geological objects and ecological systems, for the forecasting of its actions it's needed to be developed appropriate mathematical models. The process of such model creation is quite difficult and consists of several stages. Data obtained from the observations or experiments are processed by using of statistical analysis.

The development of computer technologies made it possible to apply new technologies in geology and ecology.

The article describes usage of software package Excel's tools and a number of functions in the statistical analysis.

Basic statistical indicators, such as the mean value, dispersion, asymmetry, square root dispersion, excess, etc. are located in one option, some other essential statistical characteristics can be defined by the corresponding functions (mainly by statistical and mathematical), which is described in the article in detail.

Key words: Ecology; function; geology; mathematical model; software package; statistics. 
UDC 004.942

SCOPUS CODE 1706

DOI: HTTPS://DOI.ORG/10.36073/1512-0996-2019-3-29-39

\section{Методы математического моделирования в геологии и экологии (программный пакет Excel)}

Зураб Какулия

Вера Абзианидзе

Дмитрий Абзианидзе
Институт гидрогеологии и инженерной геологии, Грузинский технический университет, Грузия, 0126, Тбилиси, село Дигоми, ул. Св. равноапостольной Нины, №1 E-mail: zukakulia@yahoo.com

Институт гидрогеологии и инженерной геологии, Грузинский технический университет, Грузия, 0126, Тбилиси, село Дигоми, ул. Св. равноапостольной Нины, №1 E-mail: veriko_abz@mail.ru

Институт гидрогеологии и инженерной геологии, Грузинский технический университет, Грузия, 0126, Тбилиси, село Дигоми, ул. Св. равноапостольной Нины, №1 E-mail: dimitri.abz@mail.ru

\section{Рецензенты:}

Н. Джапаридзе, ассистент-профессор горно-геологического факультета ГТУ

E-mail: ninojap@mail.ru

Б. Мхеидзе, главный научный сотрудник Института гидрогеологии и инженерной геологии ГТУ

E-mail: b.mkheidze@gtu.ge

Аннотация. Для описания, классификации геологических объектов и прогнозирования действий экологических систем необходимо создание их математичеких моделей. Процесс создания модели весьма сложный и состоит из нескольких этапов. Данные, полученные путем наблюдений и экспериментов, обрабатываются с применением инструментов статистического анализа.

C развитием компьютерных технологий стало возможным в геологии и экологии применять новые технологии. В статье показано, как с помощью ряда инструментов и функций программного пакета Ехсеl можно произвести статистический анализ. Основные такие статистические показатели, как среднее значение, дисперсия, квадратный корень из дисперсии, ассиметрия, эксцесс и другие сосредоточены в одной опции, другие статистические характеристики можно определить с помощью соответствующих функций. Все это показано в статье.

Ключевые слова: геология; математическая модель; программный пакет; статистика; функция; экология.

$$
\begin{aligned}
& \text { зs6bomzol osґомо 25.03.2019 }
\end{aligned}
$$

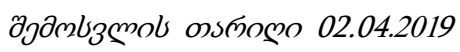

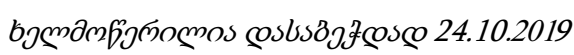




\title{
UDC 681.3
}

SCOPUS CODE 2207

DOI: https://doi.org/10.36073/1512-0996-2019-3-40-46

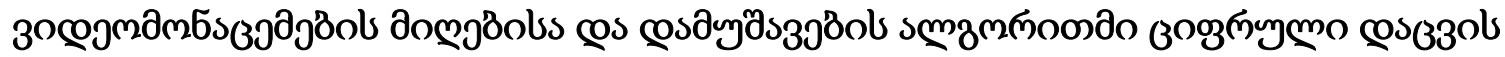

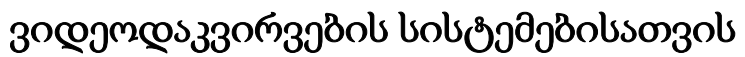

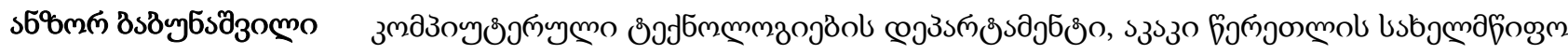

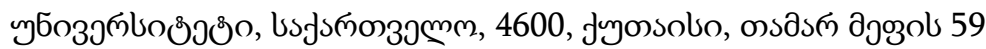 \\ E-mail: anzori.babunashvili@mail.ru \\ os dmbs

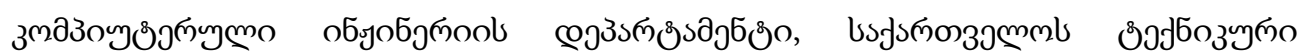

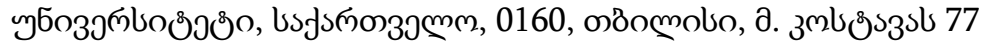 \\ E-mail: i_mosashvili@gtu.ge
}

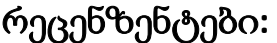

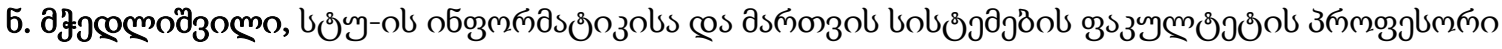

E-mail: galoba47@mail.ru

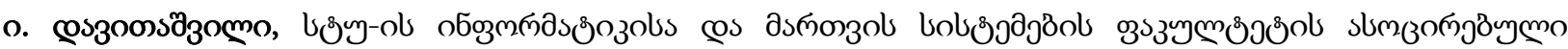
उммозуцмпо

E-mail: i.davitashvili@gtu.ge

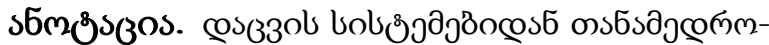

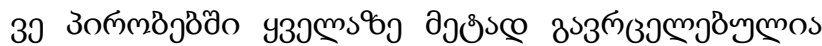

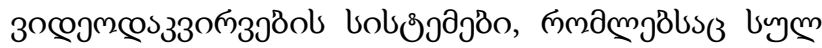

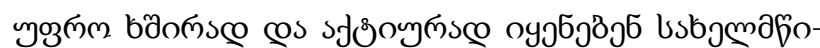

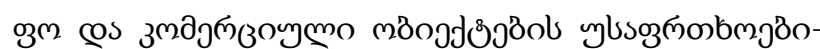

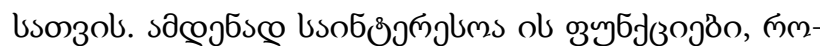

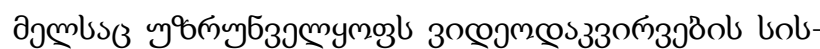

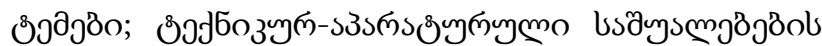

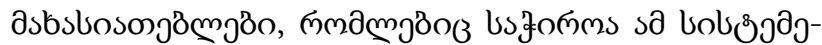

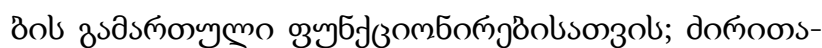

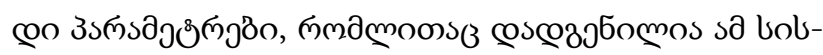

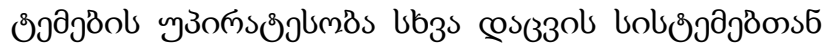

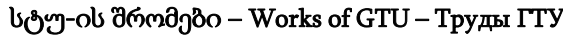
№3 (513), 2019

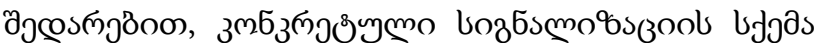

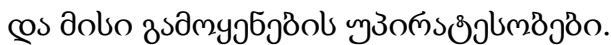

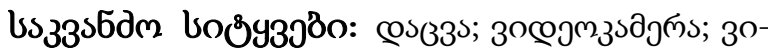

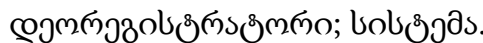

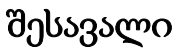

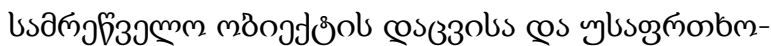

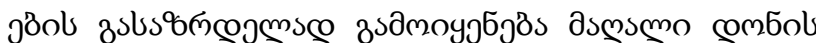

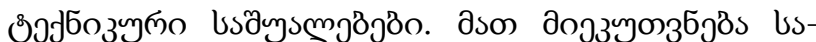

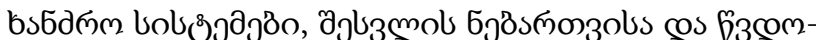

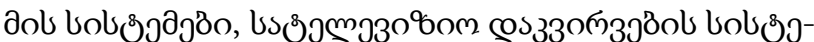

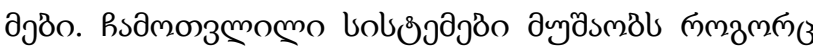




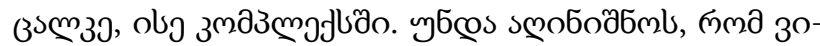

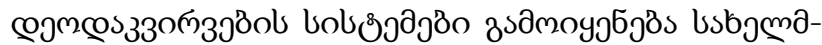

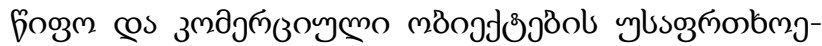

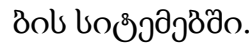

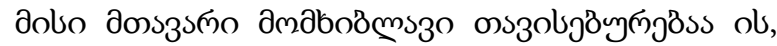

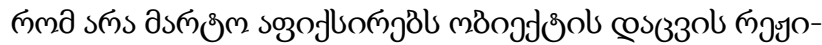

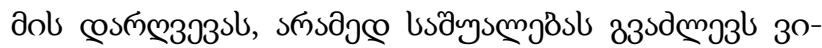

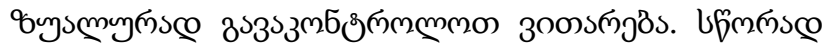

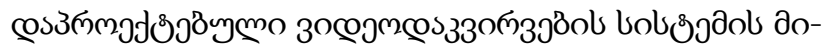

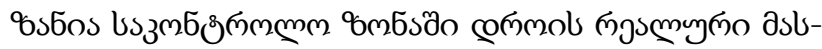

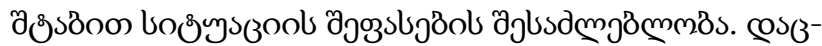

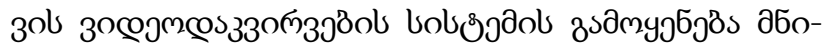

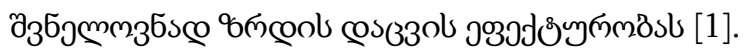

\section{domooscen Esfonmo}

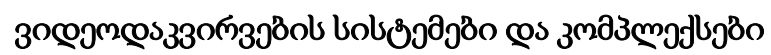

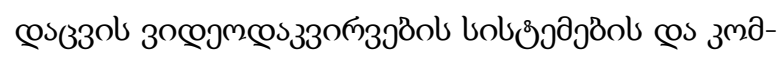

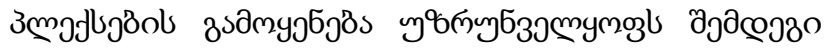

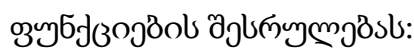

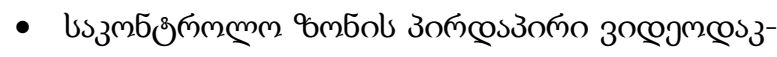

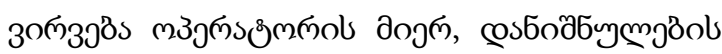

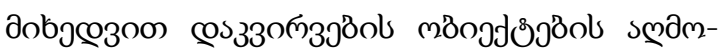

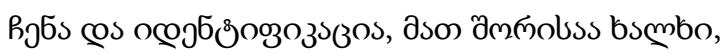

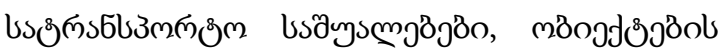

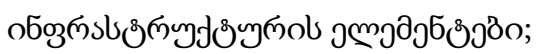

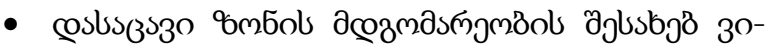

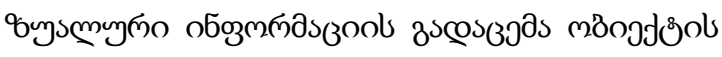

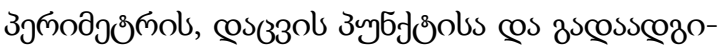

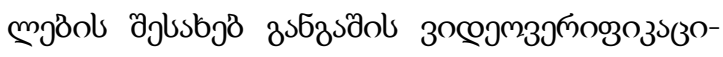

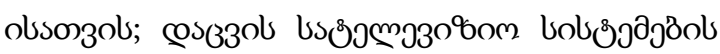

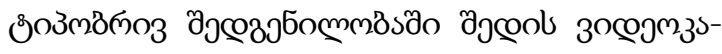

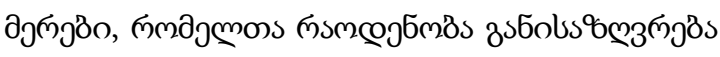

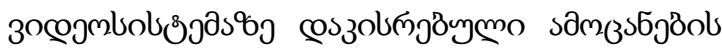

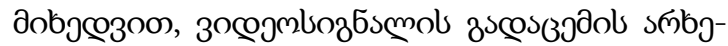

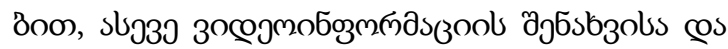

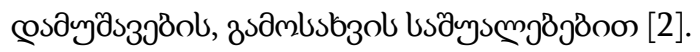

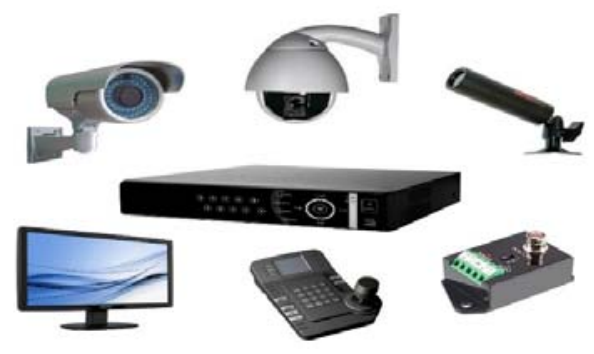

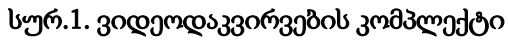

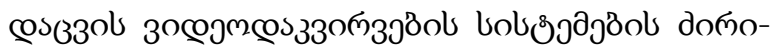

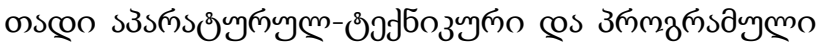

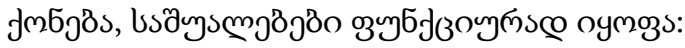

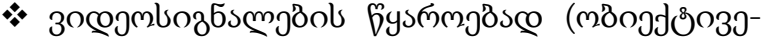

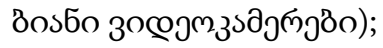

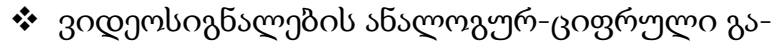

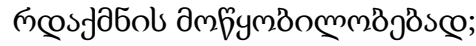

* zocombroźsmol zscoszadols cos zmanossozool bsàysmjöjösce;

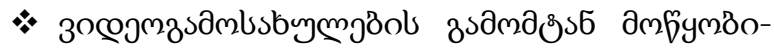

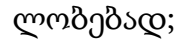

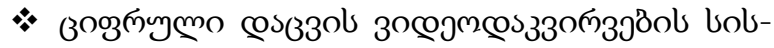

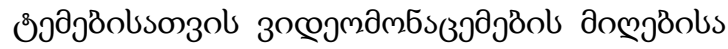

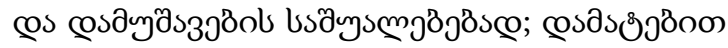

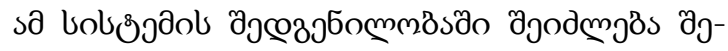

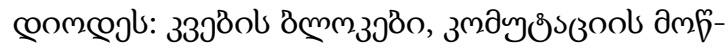

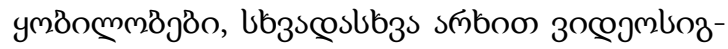

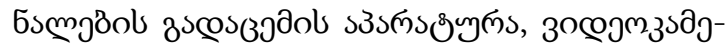

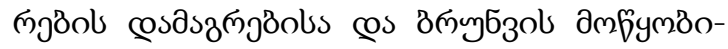

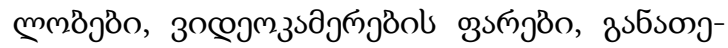

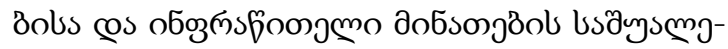

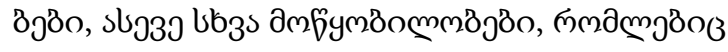




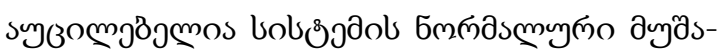
möolsonzol [2].

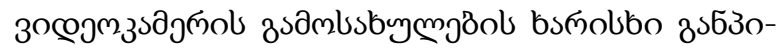

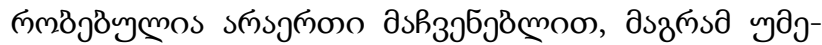

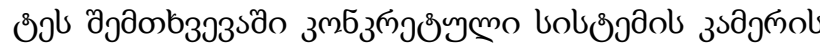

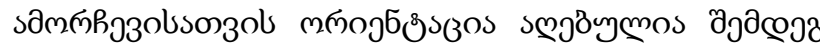

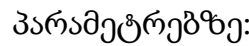

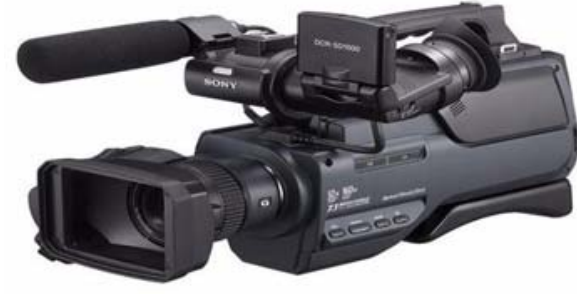

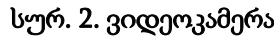

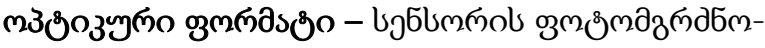

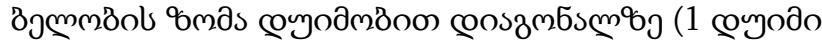

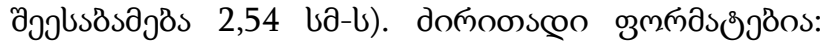

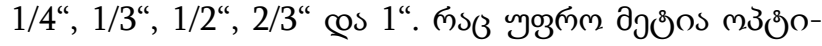

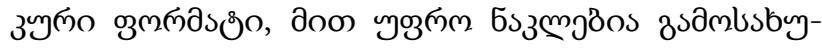

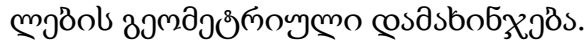

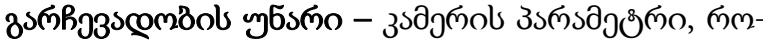

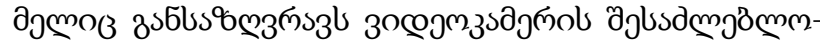

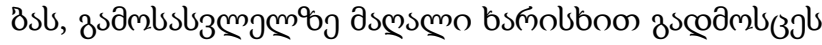

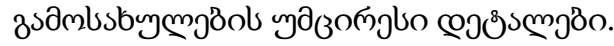

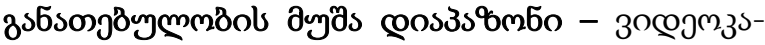

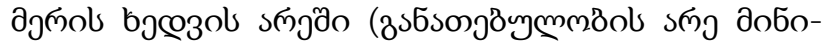

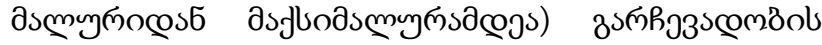

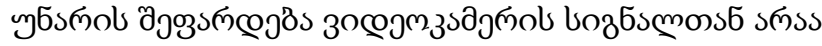

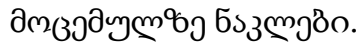

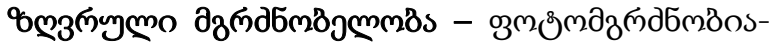

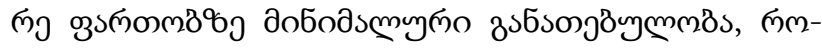

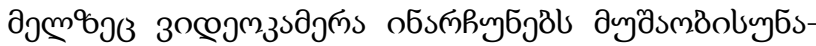
nosbmosl.

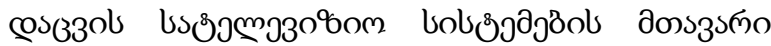

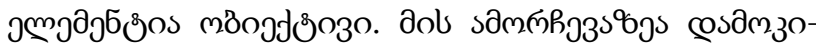

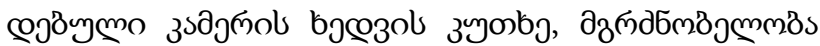

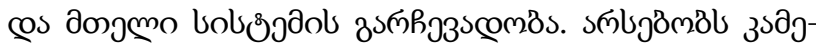

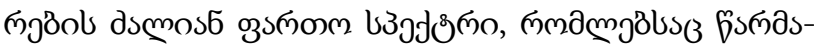

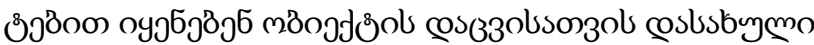

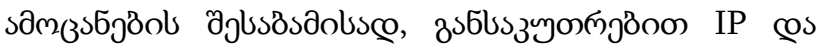

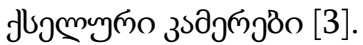

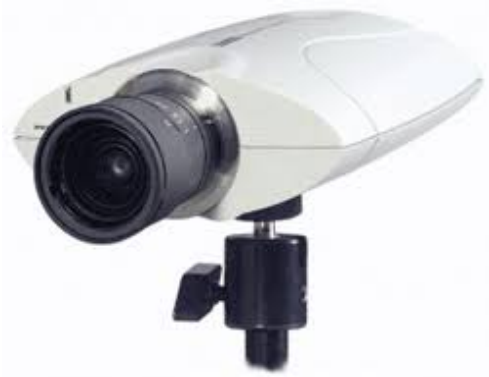

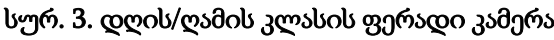

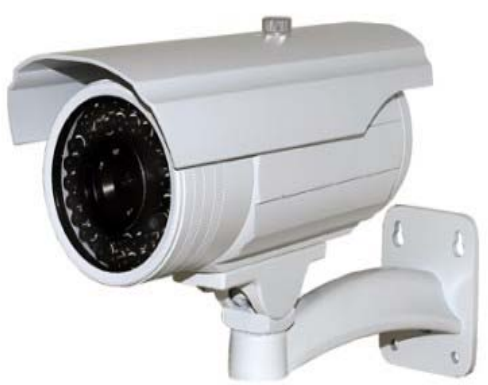

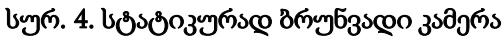

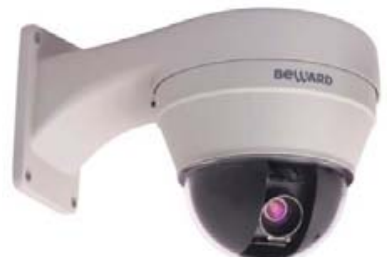

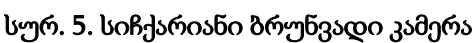




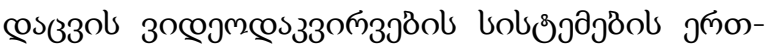

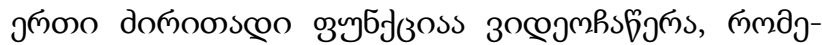

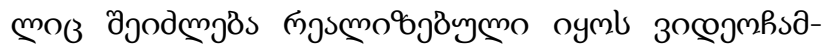

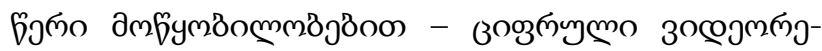

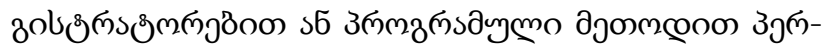

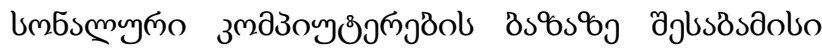

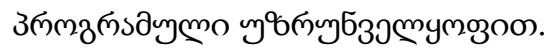

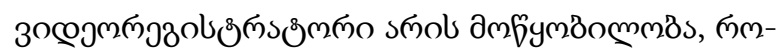

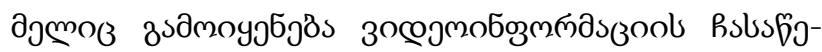

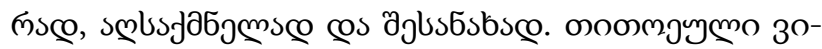

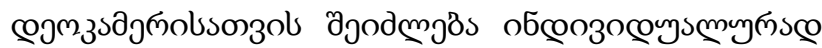

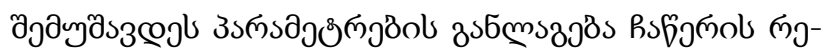
yodolsonzol.

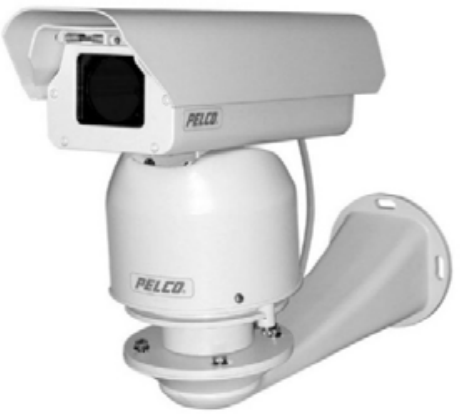

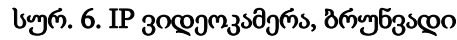

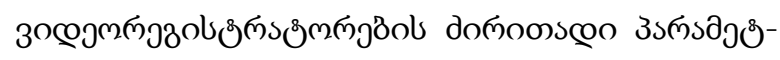
๓ூว๐ัs:

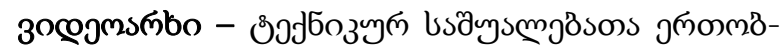

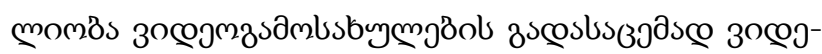

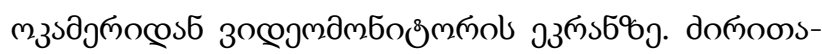

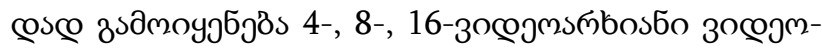

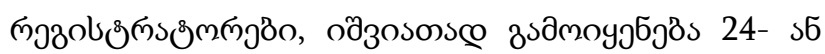
32- søbbosbo.

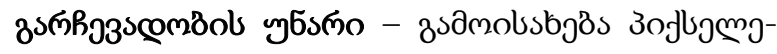

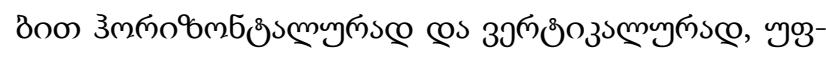

ISSN 1512-0996

www.shromebi.gtu.ge

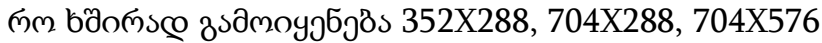
З̊नlımo.

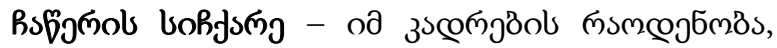

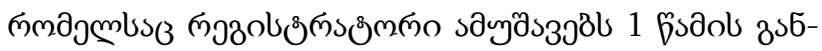

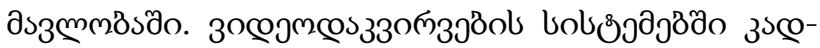

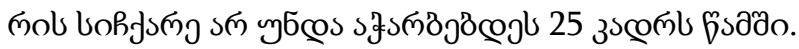

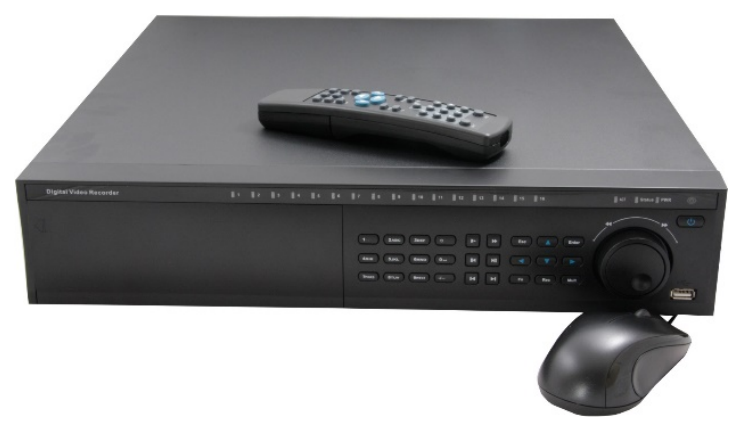

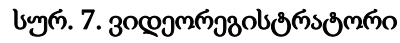

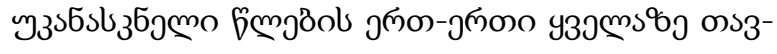

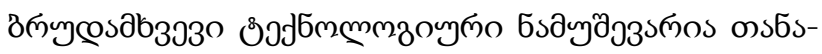

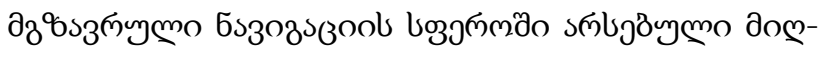

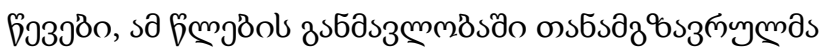

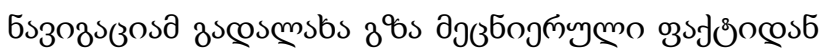

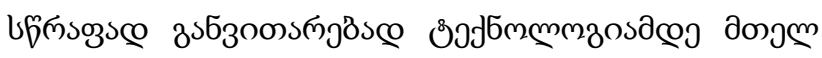

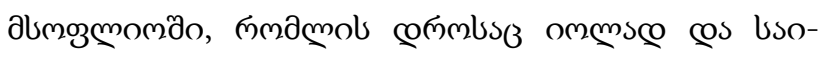

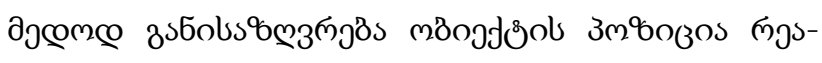
милп скмдо [3].

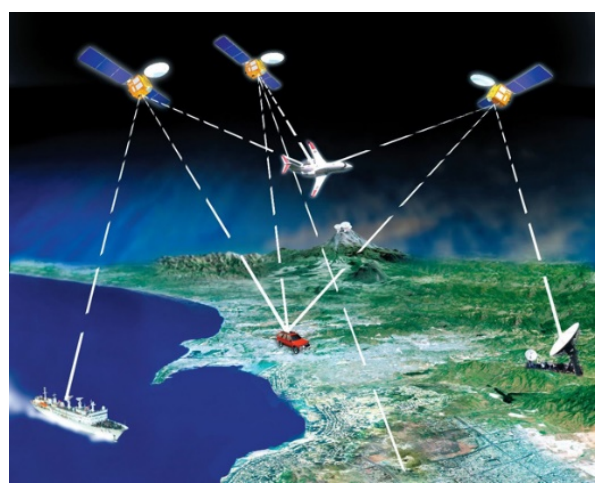

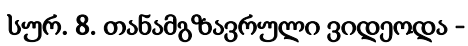

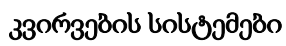

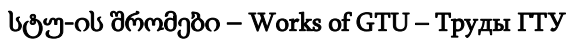
№3 (513), 2019 


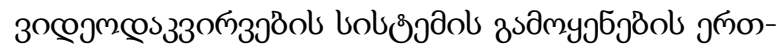

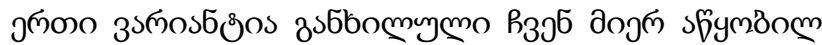

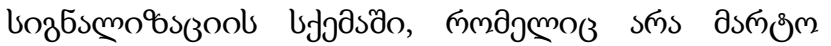

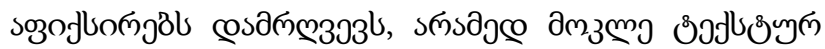

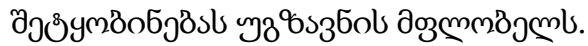

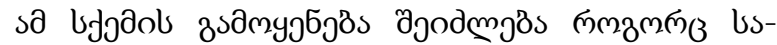

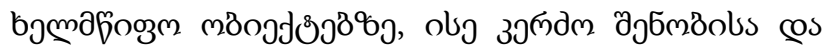

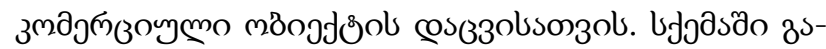

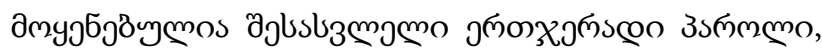

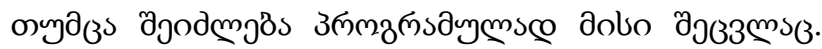

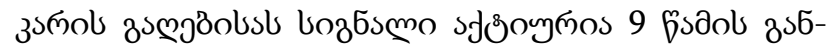

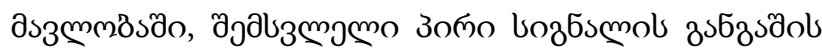

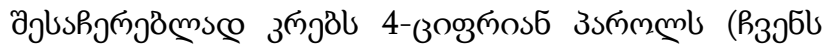

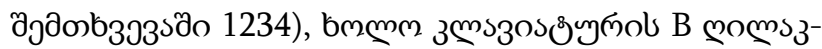

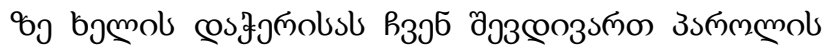

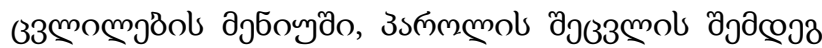

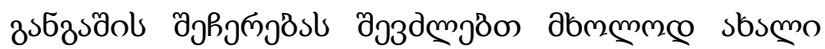

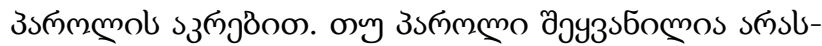

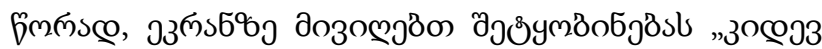
въгеgm".

\section{cosb336s}

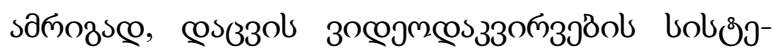

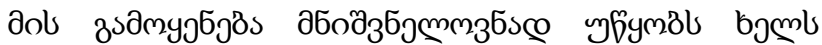

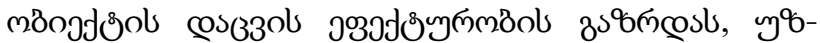

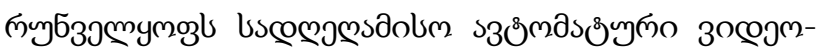

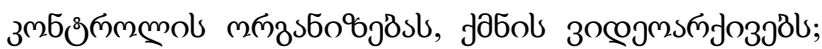

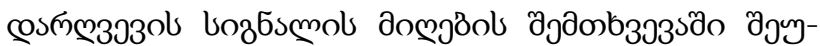

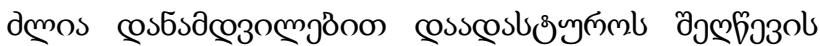

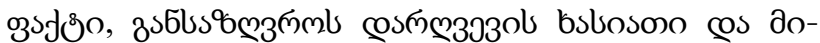

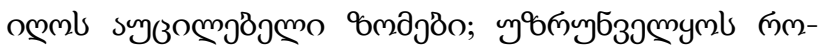

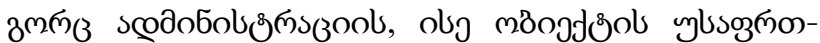

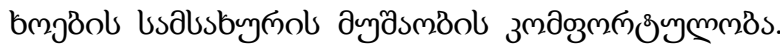

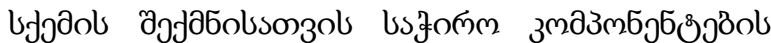

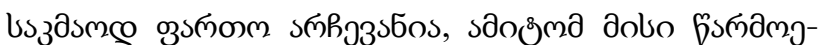

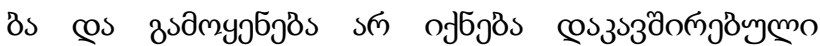

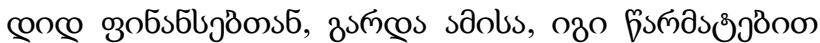

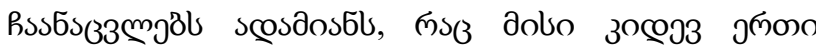
эзопьร์

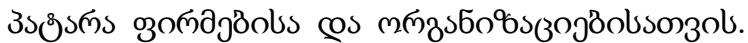

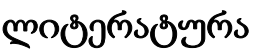

1. Volkovitsky V.D. Digital systems of TV. Sankt-Peterburg. 2012. (in Russian).

2. Gedzberg U.M. CCTV. M., 2012. (in Russian).

3. Gabdullin T.R., Zagretdinov R.V. Productivity Improvement of Machine Control Systems Using new Global Satellite Positioning Systems. Izvestiya KGASU. №4(26). Kazan. 2013, 397-402 pp. (in Russian). 


\title{
UDC 681.3
}

SCOPUS CODE 2207

DOI: https://doi.org/10.36073/1512-0996-2019-3-40-46

\section{Reception and processing algorithm of videodata for digital video surveillance system}

\author{
Anzor Babunashvili Department of Computer Technology, Akaki Tsereteli State University, 59 Tamar \\ Mepe, 4600 Kutaisi, Georgia \\ E-mail: anzori.babunashvili@mail.ru \\ Ia Mosashvili Department of Computer Engineering, Georgian Technical University, 77 M. Kostava \\ str, 0160 Tbilisi, Georgia \\ E-mail: i_mosashvili@gtu.ge
}

\section{Reviewers:}

N. Mchedlishvili, Professor, Faculty of Informatics and Control Systems, GTU

E-mail: galoba47@mail.ru

I. Davitashvili, Associate Professor, Faculty of Informatics and Control Systems, GTU

E-mail: i.davitashvili@gtu.ge

\begin{abstract}
The article considers commonly used modern video surveillance systems that are often used for state and commercial objects security. The article discusses the issues related to the functions provided by video surveillance systems; the technical characteristics of hardware and tools, which are necessary for the proper functioning of these systems; key parameters providing advanced features as well as specific alarm system and its advantages.
\end{abstract}

Key words: Security; system; videocamera; videorecorder. 


\title{
UDC 681.3
}

SCOPUS CODE 2207

DOI: https://doi.org/10.36073/1512-0996-2019-3-40-46

\section{Алгоритм приема и обработки видеоданных для цифровых охранных систем видеонаблюдения}

Анзор Бабунашвили Департамент компьютерных технологий Государственного университета Акакия Церетели, Грузия, Кутаиси, 4600, ул. Тамар мепе, 59

E-mail: anzori.babunashvili@mail.ru

Ия Мосашвили Департамент компьютерной инженерии, Грузинский технический университет, Грузия, 0160, Тбилиси, ул. М. Костава, 77

E-mail: i_mosashvili@gtu.ge

\section{Рецензенты:}

Н. Мчедлишвили, профессор факультета информатики и систем управления ГТУ

E-mail: galoba47@mail.ru

И. Давиташвили, ассоциированный профессор факультета информатики и систем управления ГТУ

E-mail: i.davitashvili@gtu.ge

Аннотация. Наиболее часто используемые из современных систем охраны системы видеонаблюдения, которые все чаще и активнее применяют для обеспечения безопасности государственных и коммерческих обьектов. Описаны те функции, которые обеспечиваются системами видеонаблюдения. Приведены технико-аппаратные характеристики, необходимые для исправного функционирования этих систем, рассмотрены основные параметры, которыми устанавливается преимущество к другим системам безопасности, обсуждается конкретная схема сигнализации и преимущества ее использования.

Ключевые слова: видеокамера; видеорегистратор; защита; система.

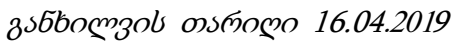

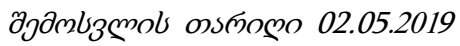

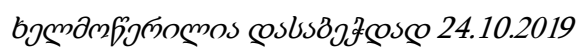




\title{
UDC 513.21
}

SCOPUS CODE 2207

DOI: https://doi.org/10.36073/1512-0996-2019-3-47-53

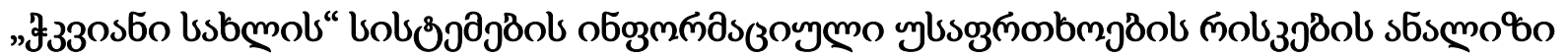

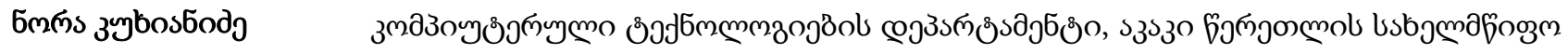

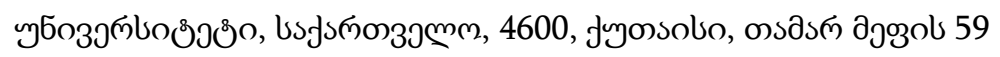 \\ E-mail: kukhianidze05nona@gmail.com

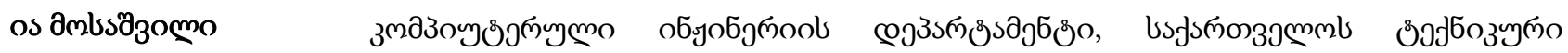

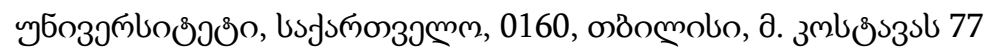 \\ E-mail: i_mosashvili@gtu.ge
}

\section{๓ว}

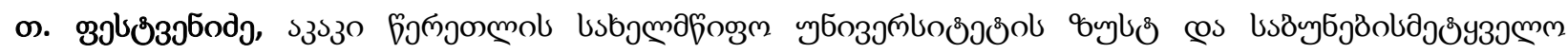

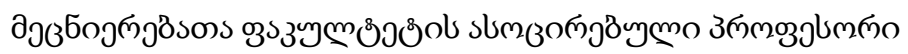

E-mail: t_pestvenidze@mail.ru

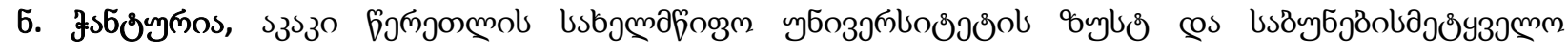

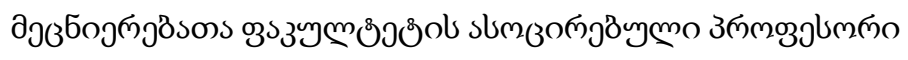

E-mail: nodarichanturia9@gmail.com

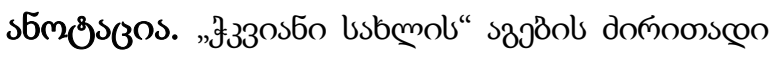

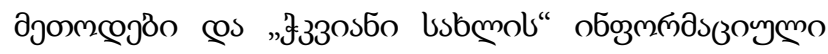

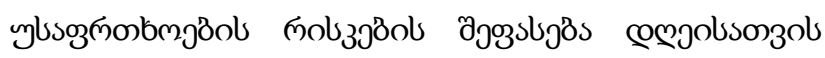

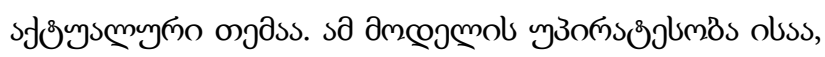

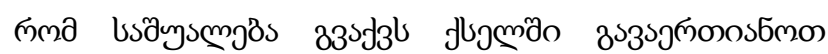

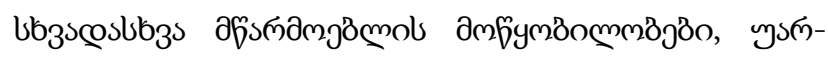

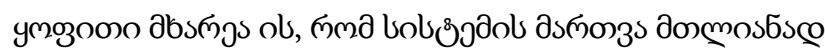

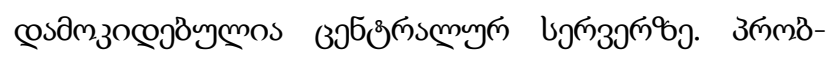

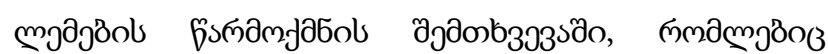

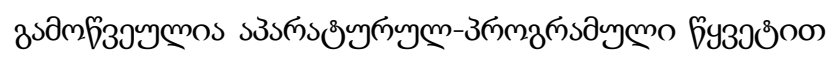

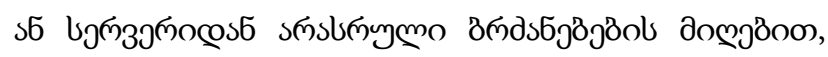

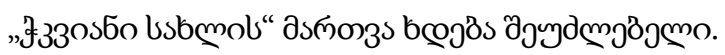

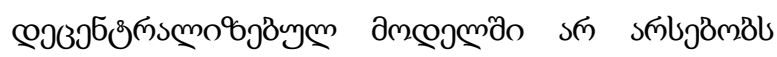

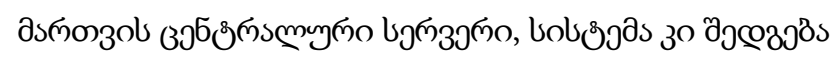

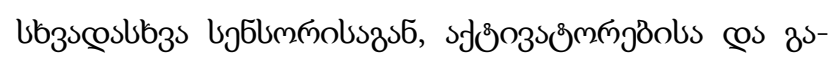

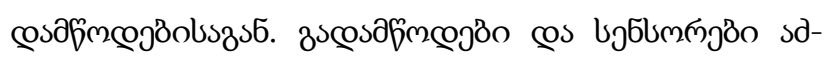

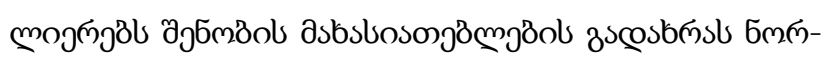

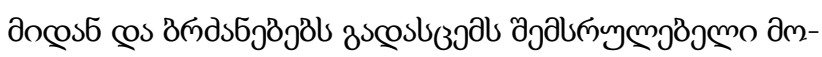

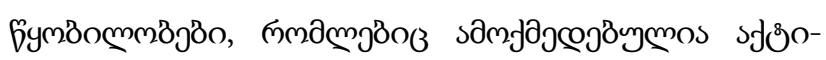

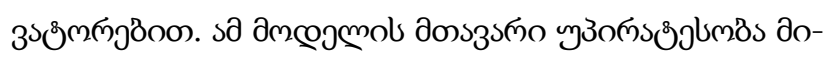

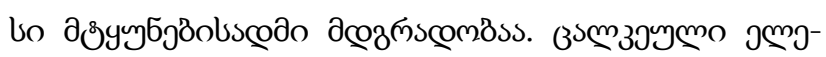

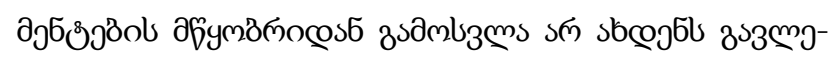




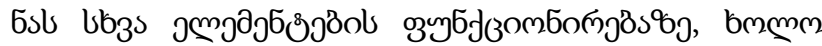

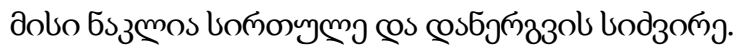

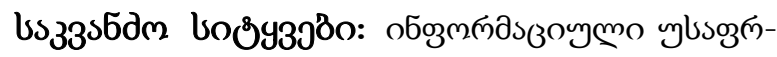

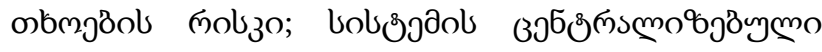

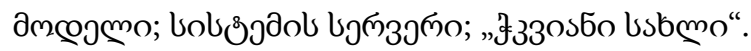

\section{gjuszsmo}

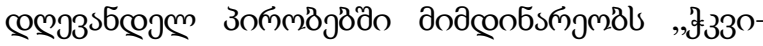

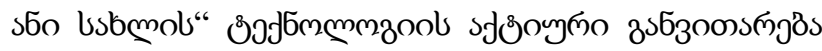

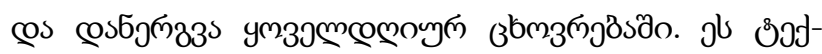

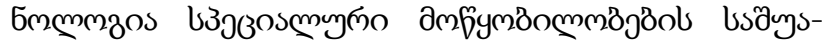

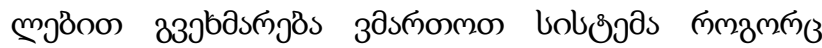

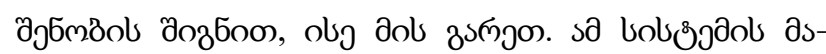

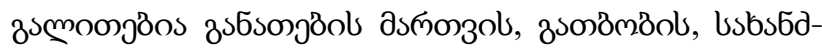

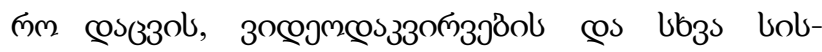

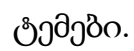

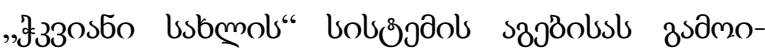

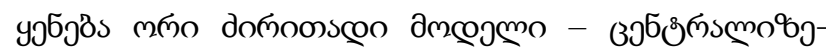

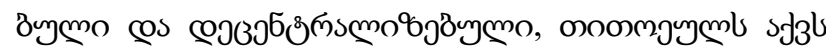

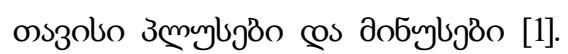

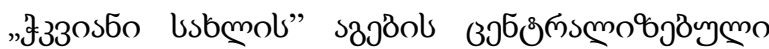
иolฮి

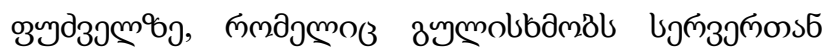

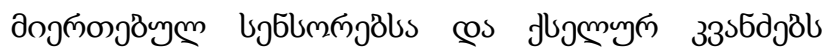

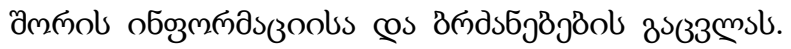

\section{domoonsceo 5sformo}

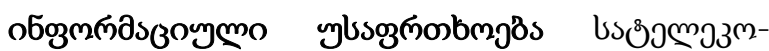

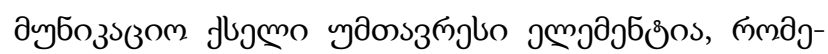

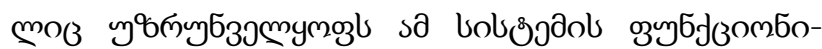

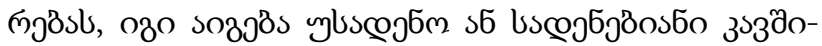

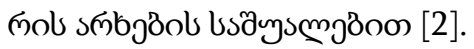

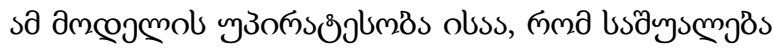

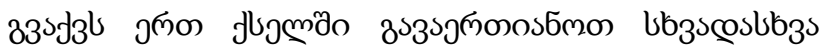

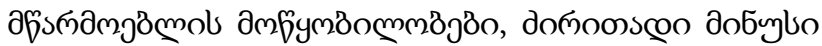
- bobsigan asmonzs, anmostsce cosamzocjäymos

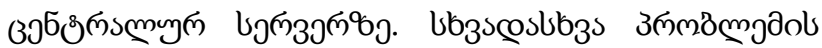

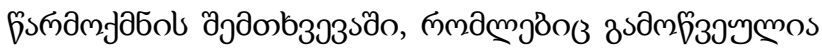

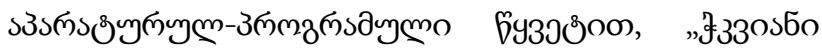

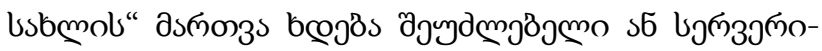

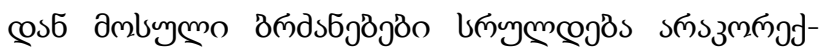
ఠ̈ymsce.

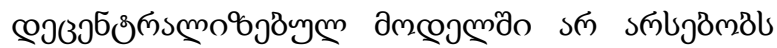

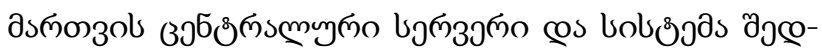

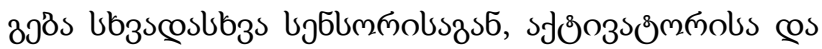

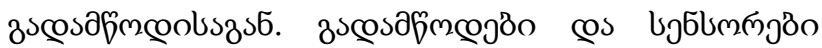

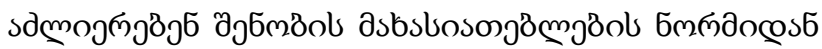

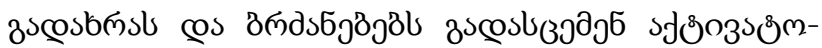

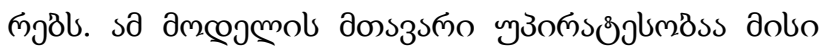

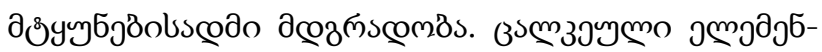

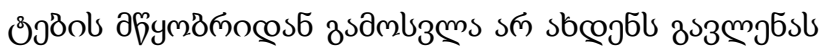

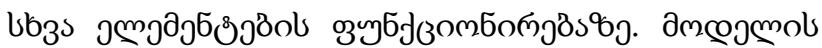

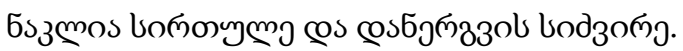

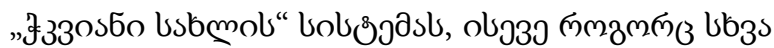

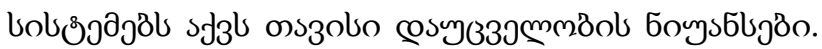

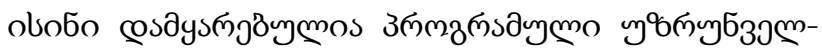

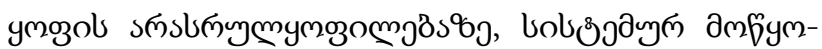

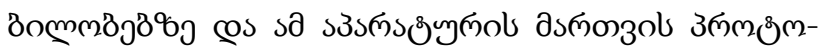

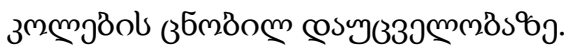

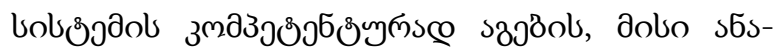

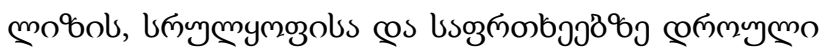

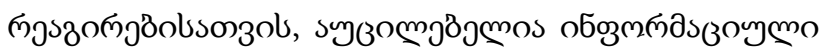

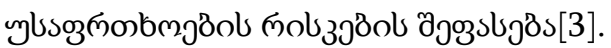




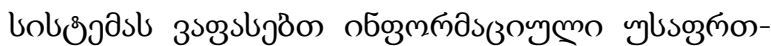

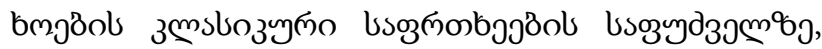
зэॅдмщ glss:

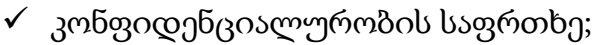

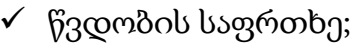

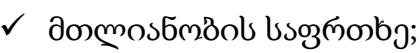

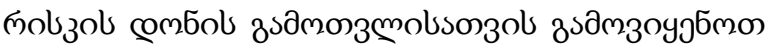

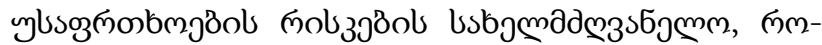

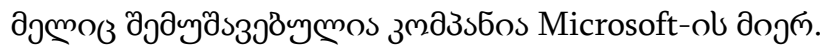

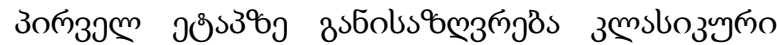

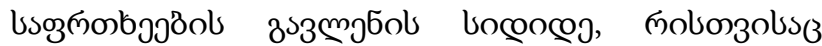

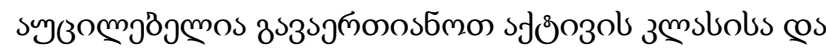

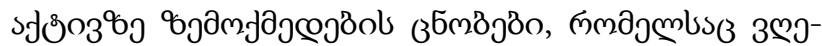

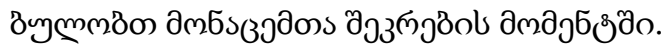

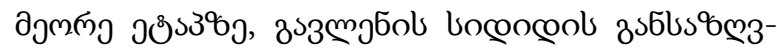

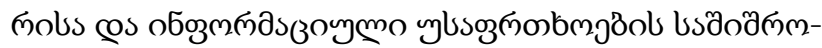

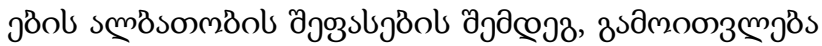
bsд̈мммм побзо.

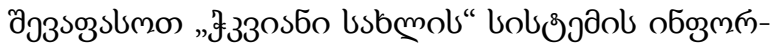

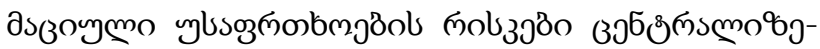

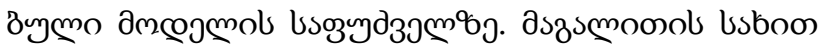

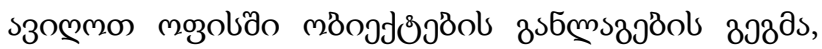

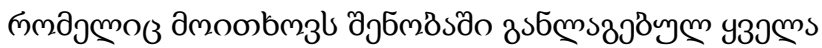

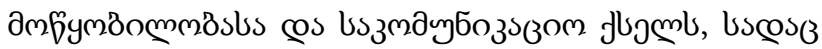

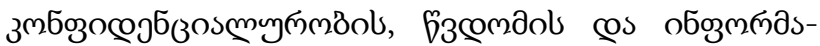

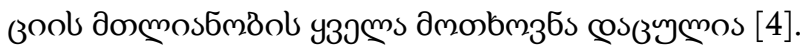

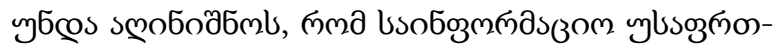
bmgöol nob

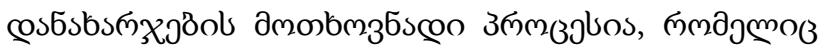

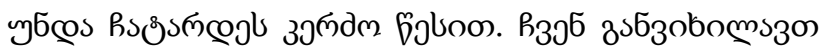

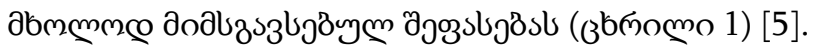

उb̆омо 1

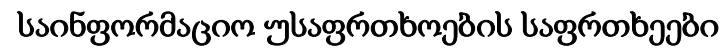

\begin{tabular}{|c|c|c|}
\hline 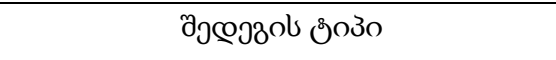 & œsy3339mm8s & ขว๕วววชั \\
\hline 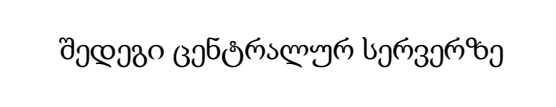 & 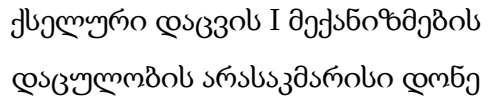 & 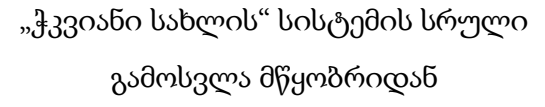 \\
\hline 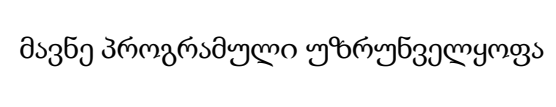 & 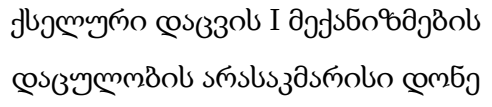 & 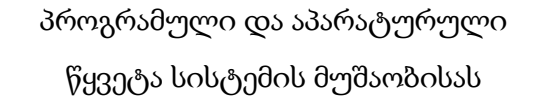 \\
\hline 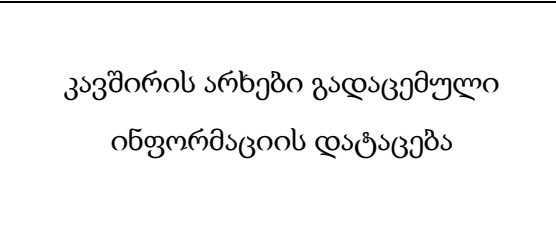 & 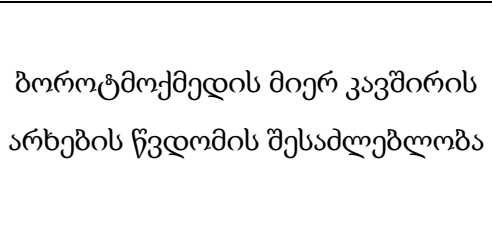 & 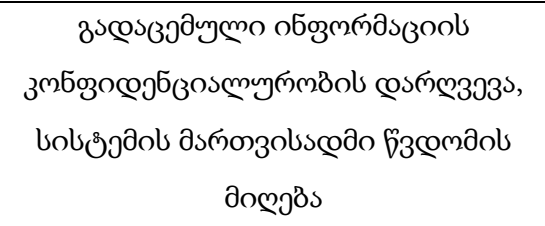 \\
\hline 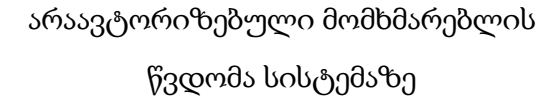 & 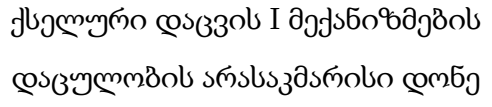 & 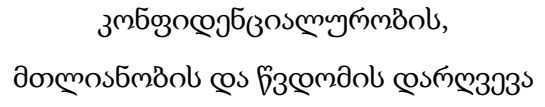 \\
\hline 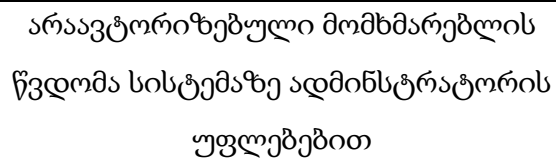 & 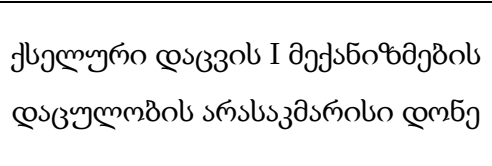 & 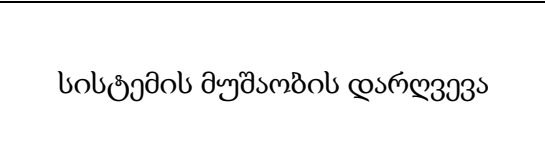 \\
\hline 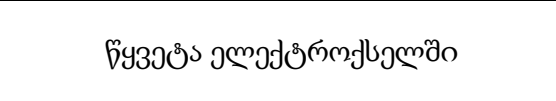 & 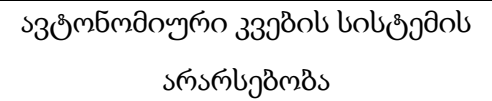 & 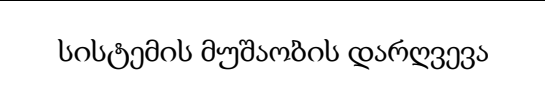 \\
\hline
\end{tabular}




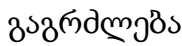

\begin{tabular}{|c|c|c|}
\hline 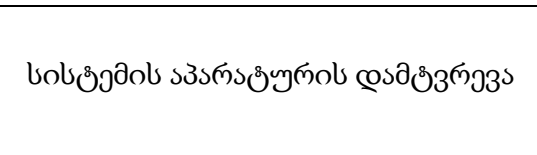 & 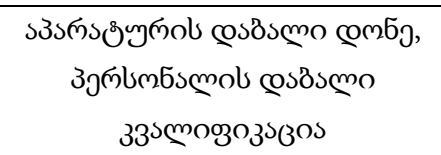 & 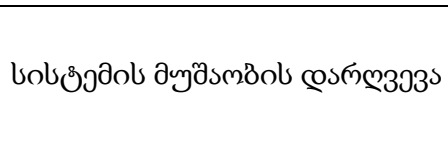 \\
\hline 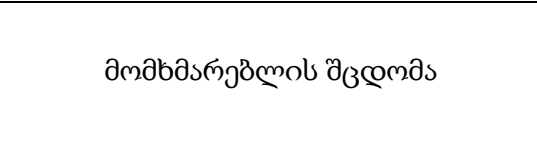 & 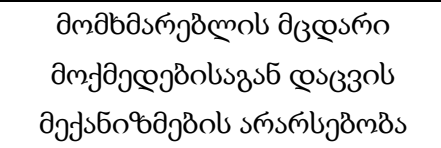 & 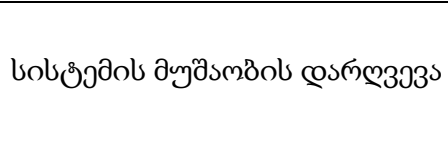 \\
\hline 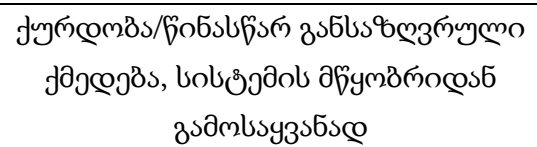 & 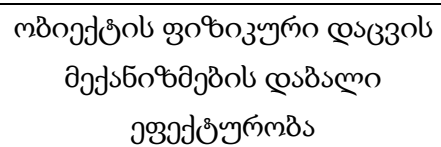 & 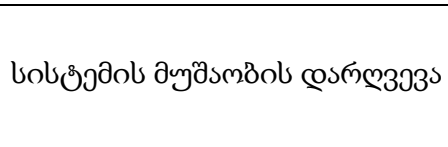 \\
\hline
\end{tabular}

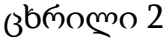

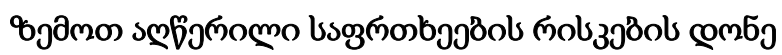

\begin{tabular}{|c|c|c|c|c|}
\hline 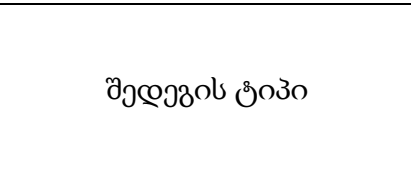 & słతింzob zmslo & $\begin{array}{l}\text { nobzols } \\
\text { comb5 }\end{array}$ & 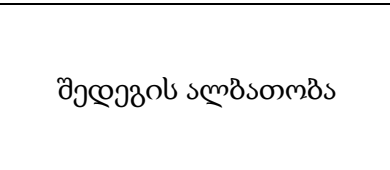 & 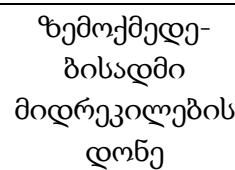 \\
\hline 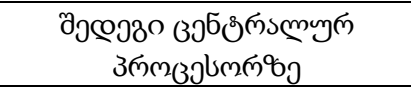 & 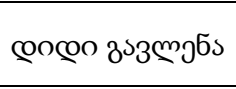 & азмьмо & дзмьмо & дзмьмо \\
\hline 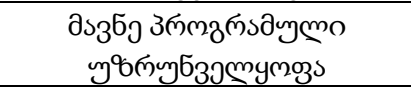 & 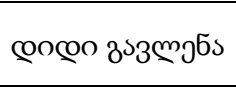 & азмзмо & asмsмmo & asçsmo \\
\hline 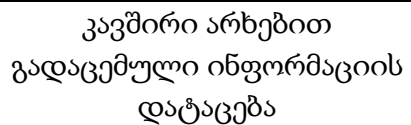 & 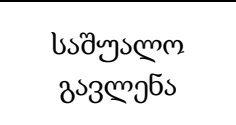 & 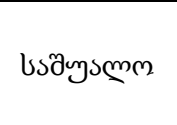 & usżульмm & bsãysmm \\
\hline 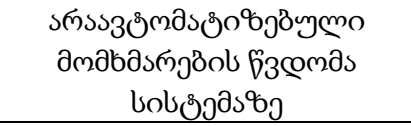 & 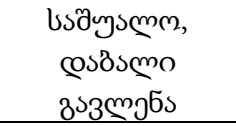 & 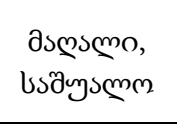 & дзмьмео & 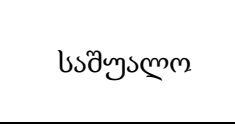 \\
\hline 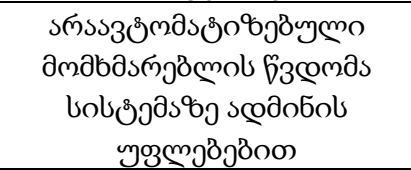 & сосо зsзm & дзмьмео & coszsemo, bszyssmm & азмьмо \\
\hline 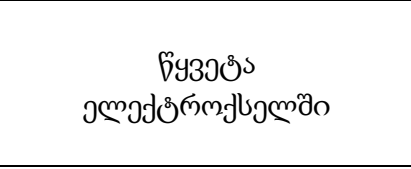 & 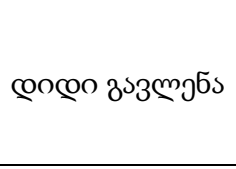 & 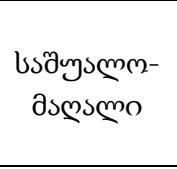 & 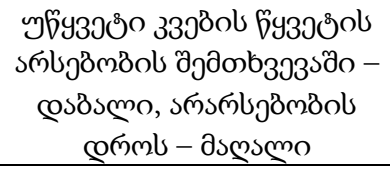 & азмьмео \\
\hline 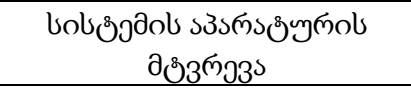 & 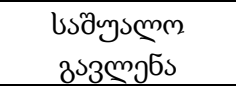 & uszаузмем & usżysmm & usżysмем \\
\hline 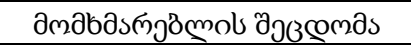 & $\partial_{3}$ о的 & bsżysmm & asmsmo & bsżysmm \\
\hline 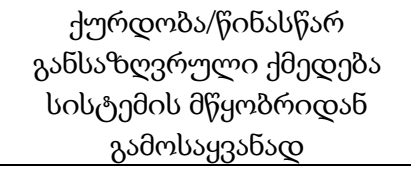 & 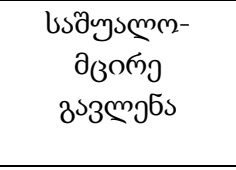 & $\begin{array}{l}\text { дsмsलmo } \\
\text { usवyssmm }\end{array}$ & uszansmем & азмьмео \\
\hline 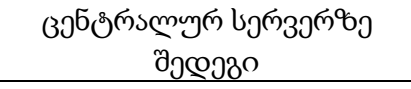 & 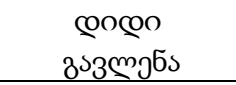 & азмзмо & дзмьмо & дзмьмо \\
\hline
\end{tabular}




\section{$\cos 33^{25}$}

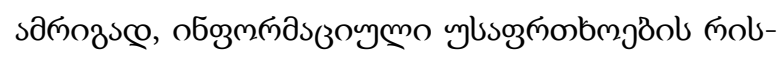

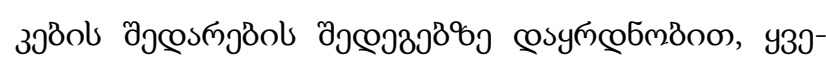

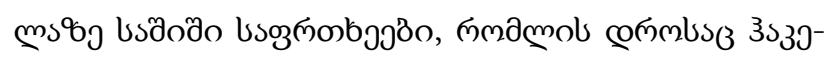

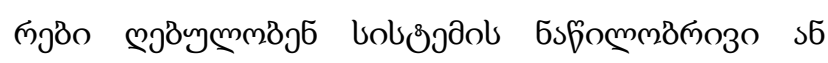

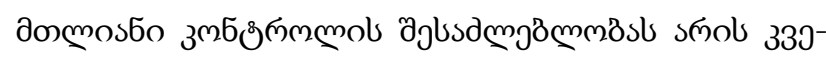

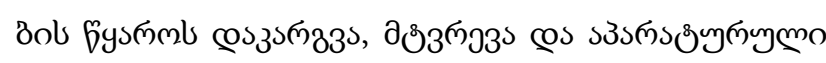

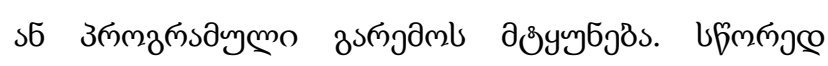

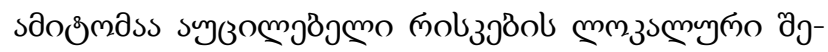

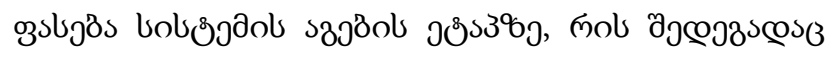

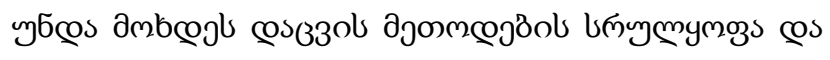

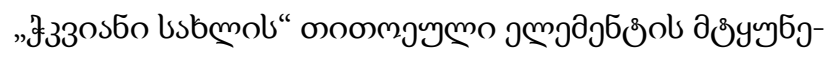

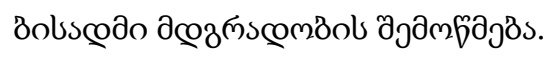

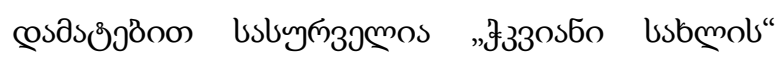

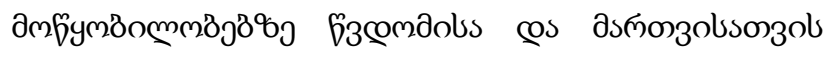

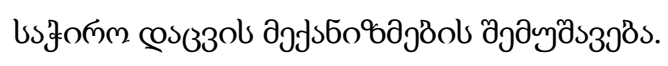

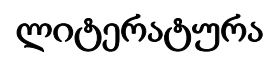

1. "Smat house" (e-resourse)-access model: /www/ URL: http://umnydom. kiev.ua/index.php?nma= catalog\&fla= stat\&cat_id=3\&page $=1 \&$ nums $=24 /-05.03 .2015$ г. - Загл. С экрана. (In Rissian).

2. Market perspectives of smart house system (e-resource)/Center of Engineering technologies CENTEC.ACCESS mode: /www/ URL: http://www. Centec group.ru/press/articles/18/ - 02.03.2011 г. - Загл. С экрана. (In Rissian).

3. Girak P. Intellectual building: why we pay mony? [Text]/P.Girak//Yournal.S.M.A.R.T. - 2018-\#2-p.8-12. (In Rissian).

4. Kurmich A. Tireless Worker. Syste, "Smart house" [Text]/kuzmich//yournal S.M.A.R.T. - 2017 - -\#2. pages 10-13. (In Rissian).

5. Cetegories "Smart House" (e-resource)/Bussines house - access mode: /www/ URL: http://ruswires.net/ post_1267720042.html/ — 04.03.2016 г. — Загл. С экрана. (In Rissian).

ISSN 1512-0996

www.shromebi.gtu.ge 


\section{UDC 513.21}

SCOPUS CODE 2207

DOI: https://doi.org/10.36073/1512-0996-2019-3-47-53

\section{Infosecuty risk analysis for "Smart House" systems}

Nora Kukhianidze Department of Computer Technology, Akaki Tsereteli State University, 59 Tamar Mepe, 4600 Kutaisi, Georgia

E-mail: kukhianidze05nona@gmail.com

Ia Mosashvili Department of Computer Engineering, Georgian Technical University, 77 M. Kostava str, 0160 Tbilisi, Georgia

E-mail: i_mosashvili@gtu.ge

\section{Reviewers:}

T. Pestvenidze, Associate Professor, Department of Computer Technology, ATSU

E-mail: t_pestvenidze@mail.ru

N. Chanturia, Associate Professor, Department of Computer Technology, ATSU

E-mail: nodarichanturia9@gmail.com

Abstract. The article discusses basic methods of design a "Smart House" and analyzes the infosecurity risks. The advantage of this model is that we can combine different manufacturers' devices in a single network, the main disadvantage is - the system management depends entirely on the central server. In case of various problems caused by a hardware-program breakdown, the "Smart House" is impossible to manage or the orders from the server are inaccurate.

In the decentralized model there is no central server management and the system consists of different sensors, activators and transmitters. The sensors and transmitters enhance building performance characteristics deviation from the rated value and deliver orders to the actuation devices that are activated by activators. The main advantage of this model is its stability against failure. Failures of separate elements don't affect the functioning of other elements. The disadvantage is its complexity and high cost implementation.

Key words: Centralized system model; infosecuty risk; server system; Smart House. 


\section{UDC 513.21}

SCOPUS CODE 2207

DOI: https://doi.org/10.36073/1512-0996-2019-3-47-53

\section{Анализ рисков информационной безопасности для систем «Умный дом»}

Нора Кухианидзе Департамент компьютерных технологий, Государственный университет им. Акакия Церетели, Грузия, 4500, Кутаиси, ул. Тамар Мепе, 59

E-mail: kukhianidze05nona@gmail.com

Ия Мосашвили Департамент компьютерной инженерии, Грузинский технический университет, Грузия, 0160, Тбилиси, ул. М. Костава, 77

E-mail: i_mosashvili@gtu.ge

\section{Рецензенты:}

Т. Пественидзе, ассоц. профессор факультета точных и ествественных наук Государственного университета им. Акакия Церетели

E-mail: t_pestvenidze@mail.ru

Н. Чантурия, ассоц. профессор факультета точных и естественных наук Государственного университета им. Акакия Церетели

E-mail: nodarichanturia9@gmail.com

Аннотация. В статье рассматриваются основные методы построения «Умного дома», анализируются риски информационной безопасности «Умного дома». Преимущество этой модели в том, что мы можем обьединять устройства разных производителей в одной сети, главный недостаток - управление системой полностью зависит от центрального сервера. В случае возникновения различных проблем, вызванных сбоем аппаратно-программных средств, управление “Умным домом» становится невозможным или заказы, полученные от сервера, выполняются некорректно.

В децентрализованной модели отсутствует централизованное управление сервером, а система состоит из разных датчиков, активаторов и передатчиков. Передатчики и датчики улучшают рабочие характеристики здания от нормы и доставляют заказы на устройство-исполнители, которые приводятся в действие активаторами. Основным преимуществом этой модели является ее устойчивость от сбоев. Сбои отдельных элементов не влияют на функционирование других элементов, а недостаток- на сложность и дороговизну внедрения.

Ключевые слова: модель централизованной системы; риск информационной безопасности; сервер системы; «Умный дом».

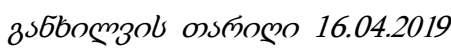

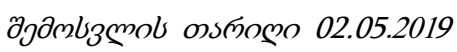

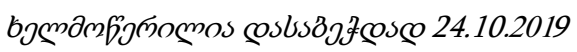


UDC 620.16

SCOPUS CODE 2209

DOI: https://doi.org/10.36073/1512-0996-2019-3-54-60

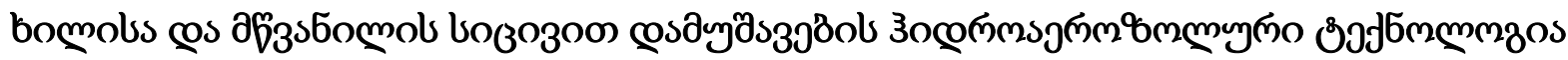

zos zmmjososto

osas\% olszsdo

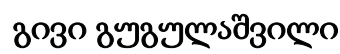

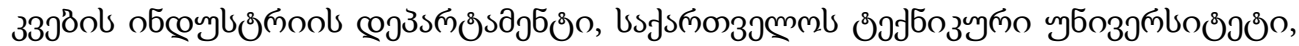

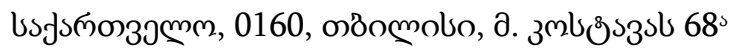

E-mail: gia_goletiani@yahoo.com

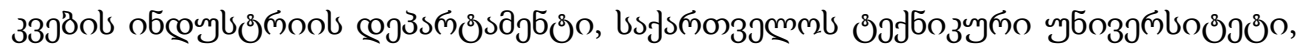

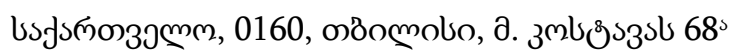

E-mail: tamazisakadze@gmail.com

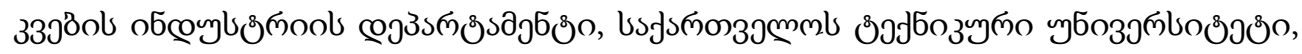

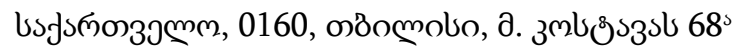

E-mail: Givi.Gugulashvili@gmail.com

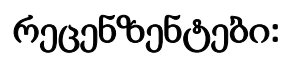

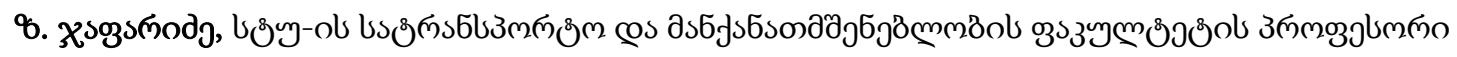

E-mail: zurabjaparidze@yahoo.com

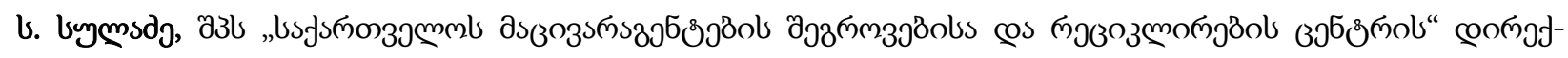

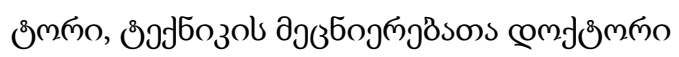

E-mail: sulkhansuladze@gmail.com

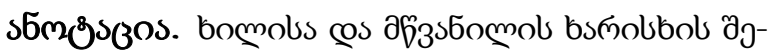

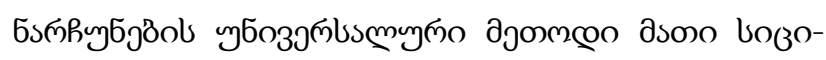

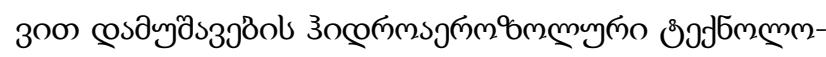

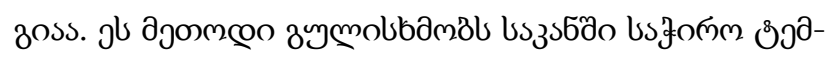

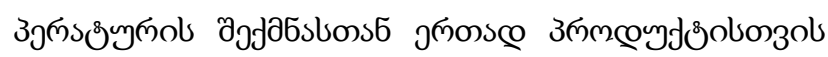

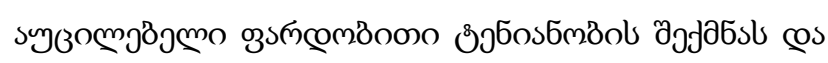

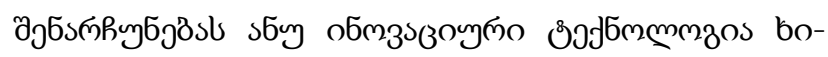

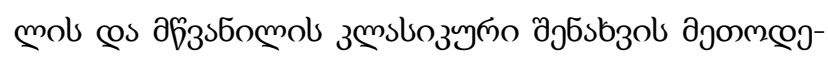

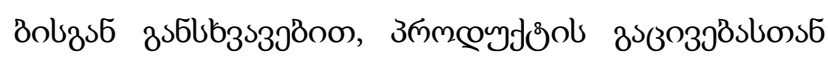

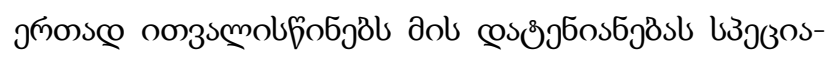

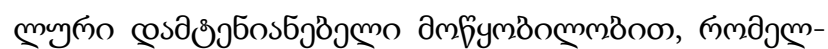

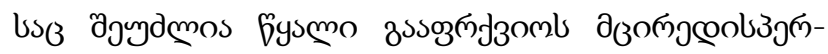

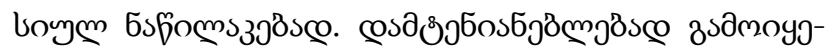

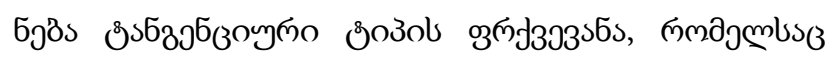

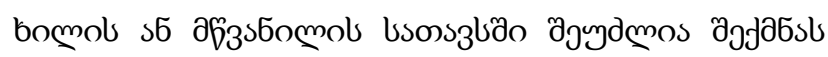

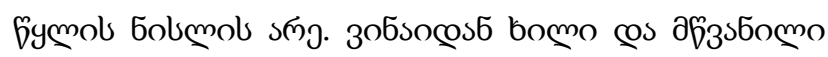

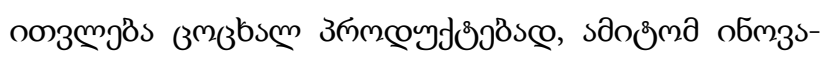

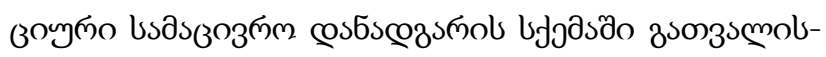

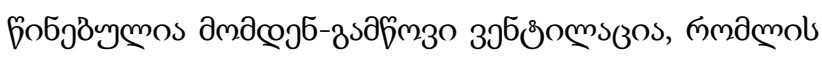

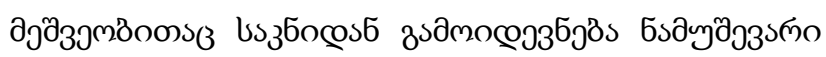

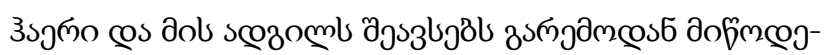




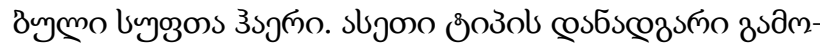

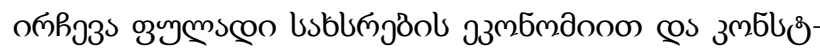

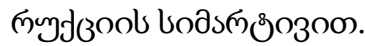

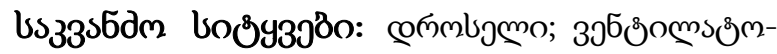

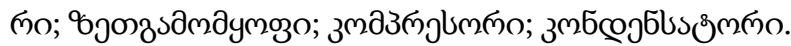

\section{gjuszsco}

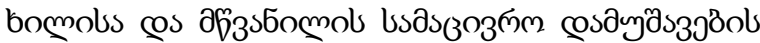

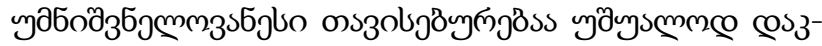

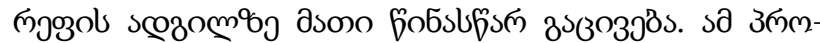

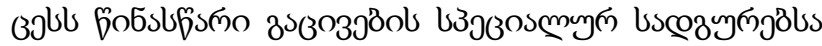

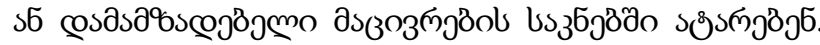

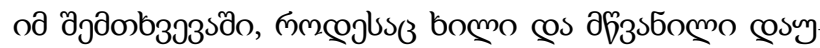

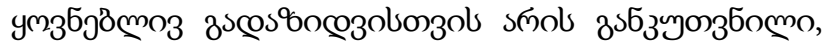

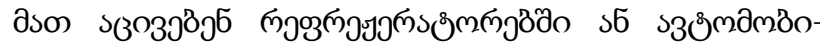

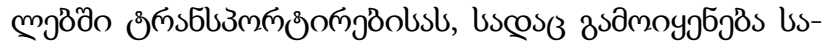

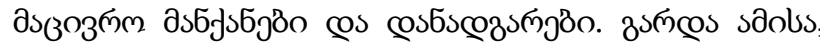

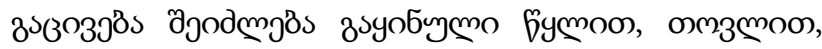

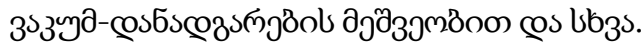

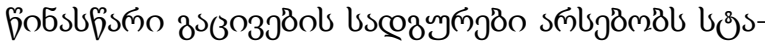

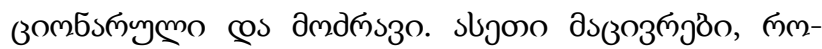

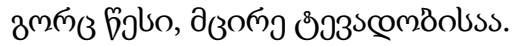

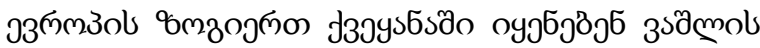

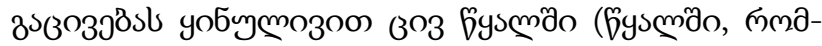

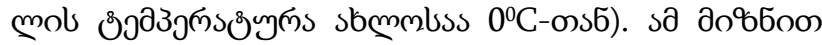

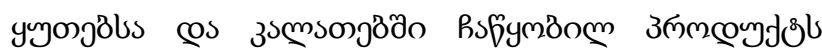
smsз

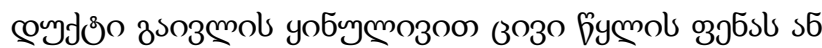

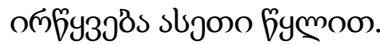

\section{domooscen 6sfomo}

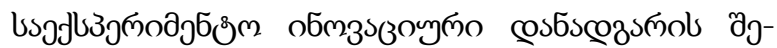

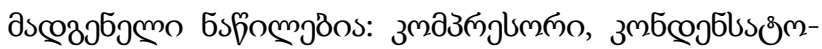

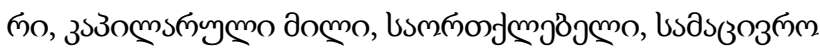

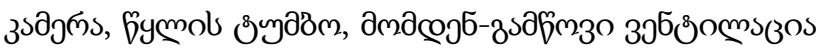

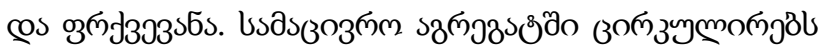

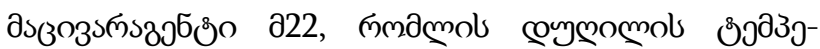

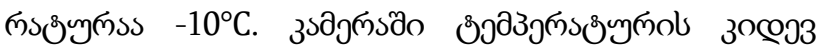

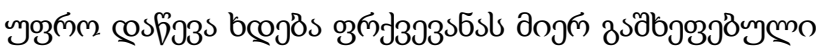

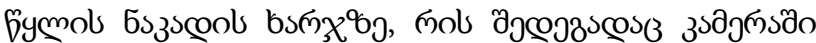

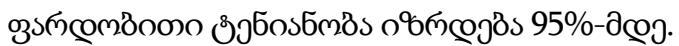

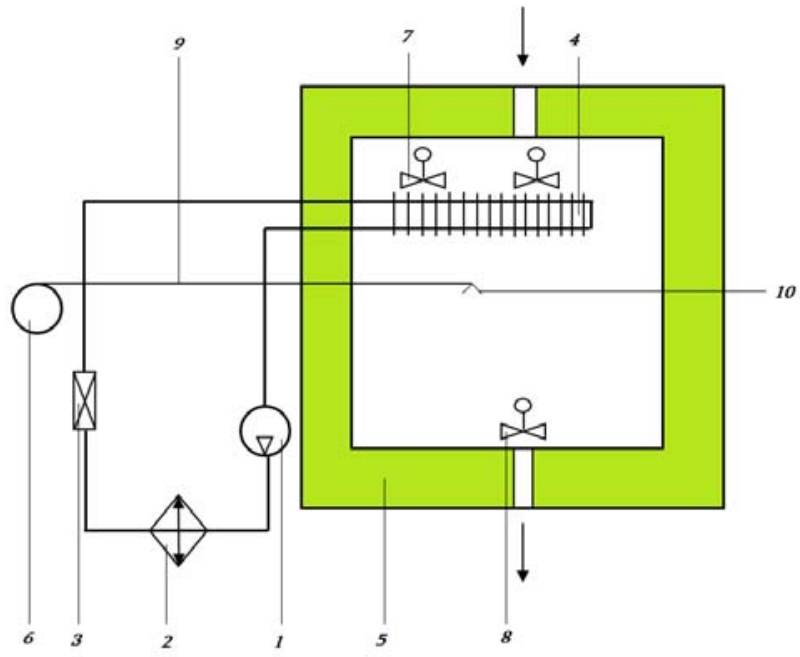

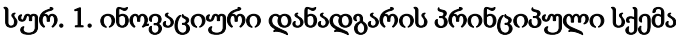

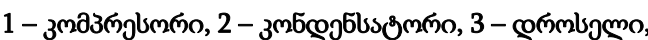

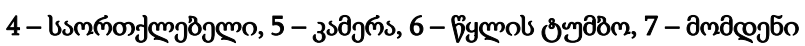

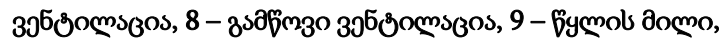

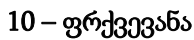

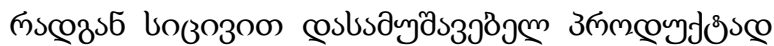

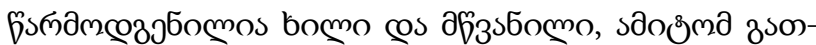

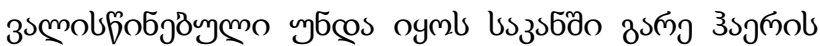

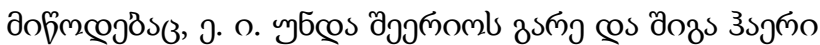

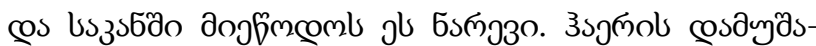
зј

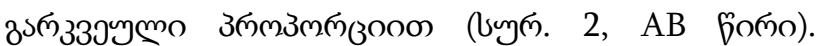




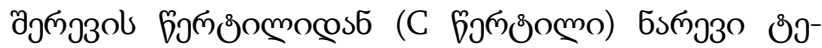

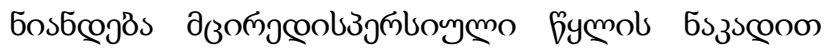

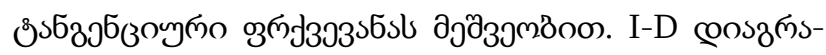

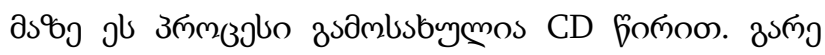

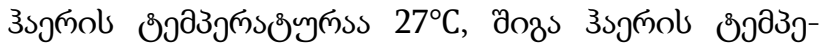

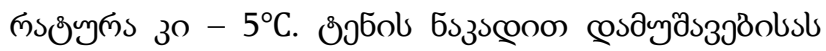

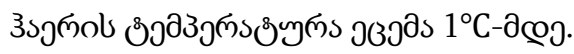

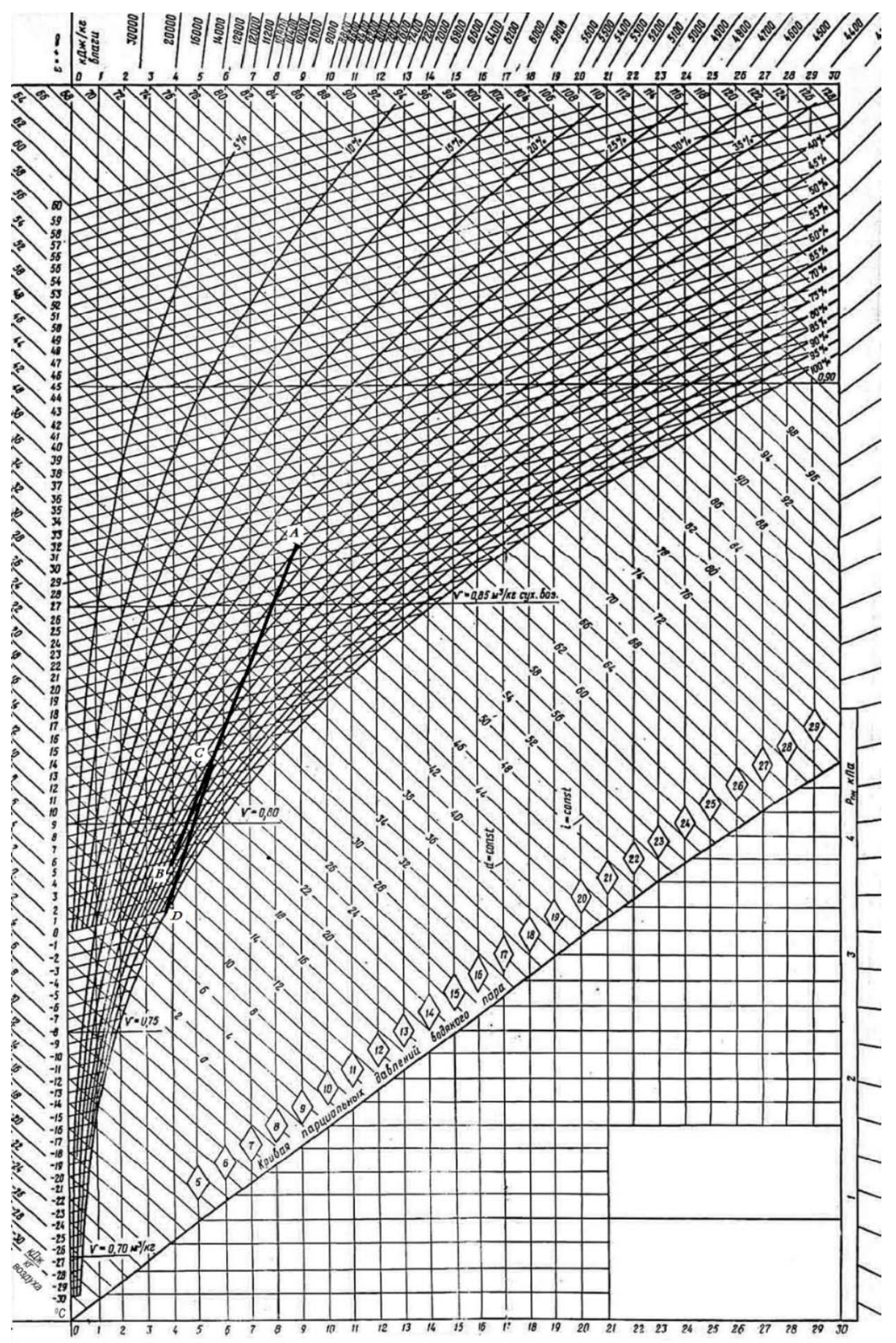

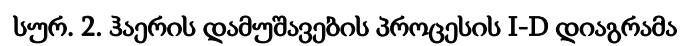




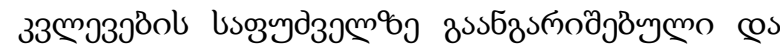

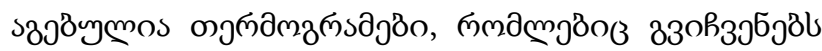

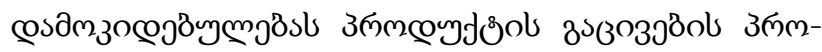

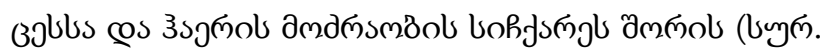
3 cos byn. 4).

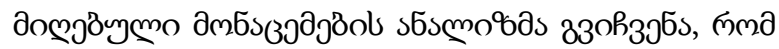

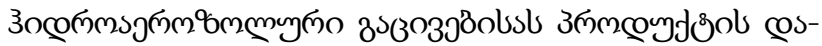

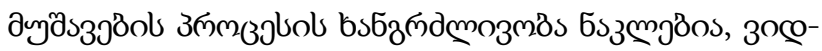

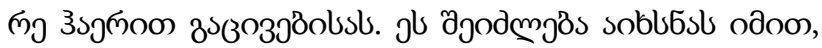

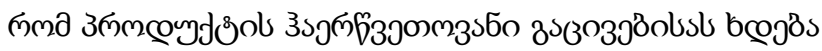

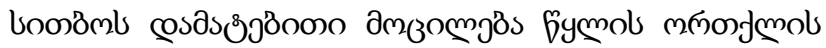
оз

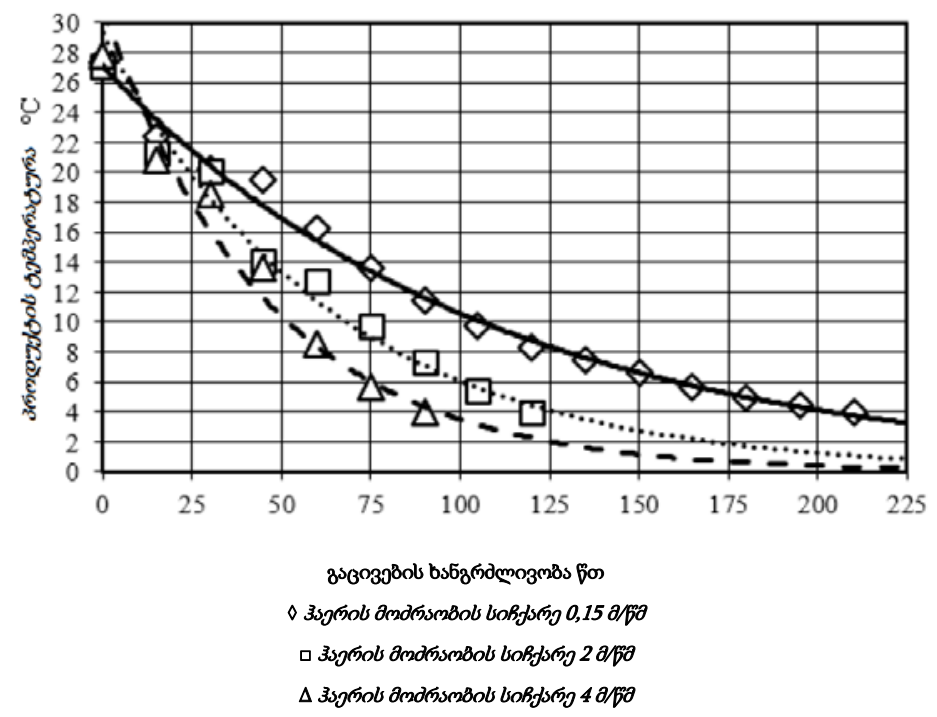

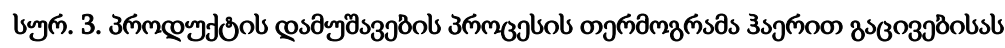

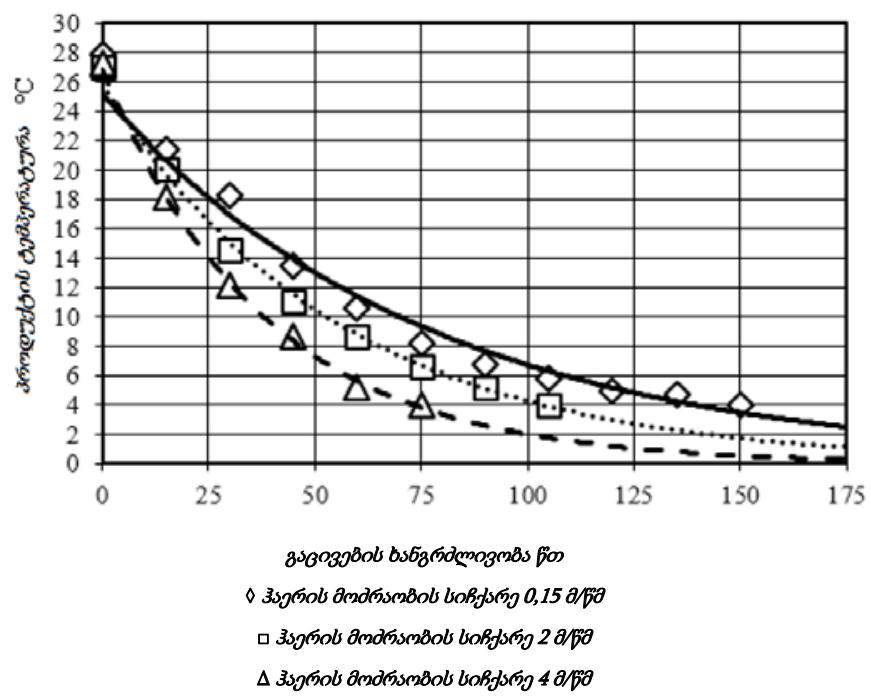

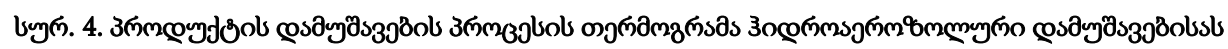




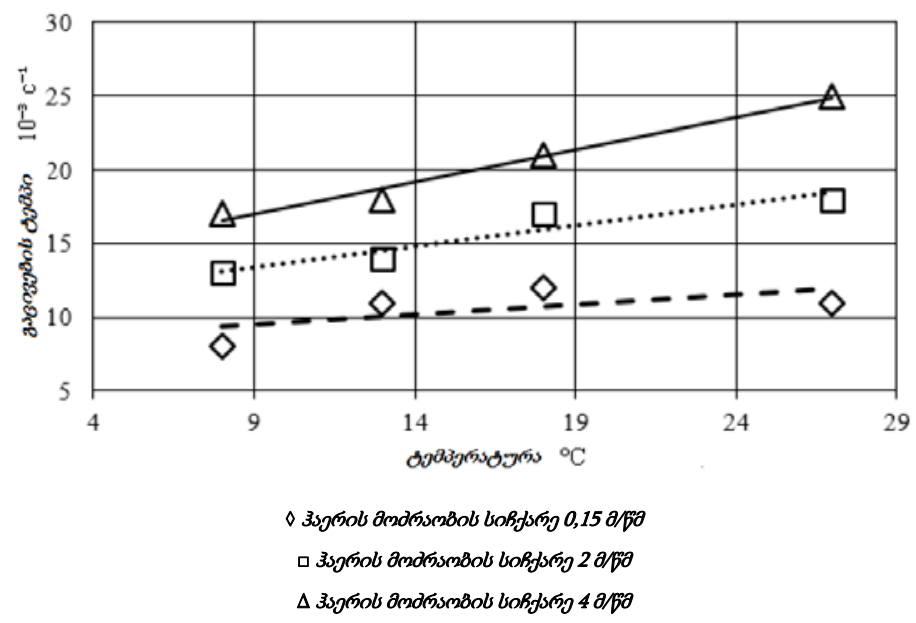

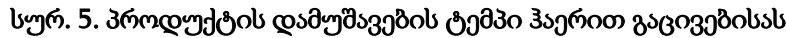

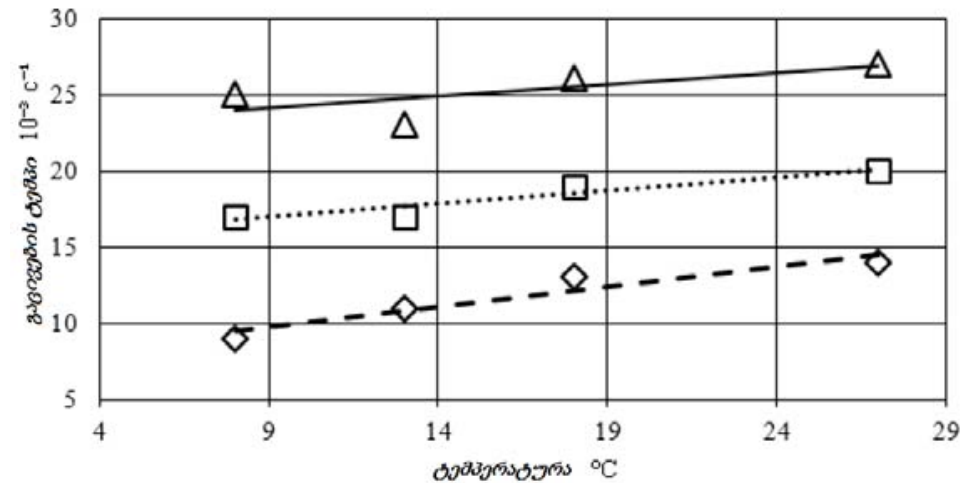

$\checkmark$ 3งวตั

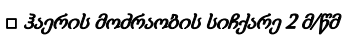

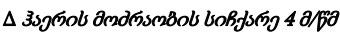

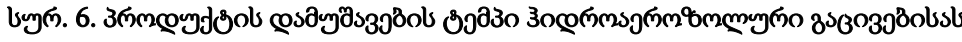

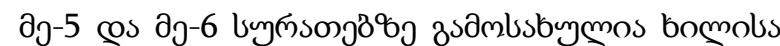

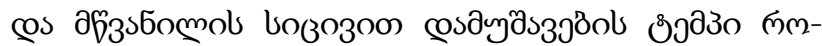

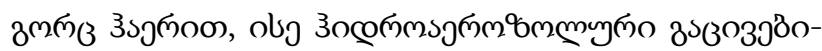

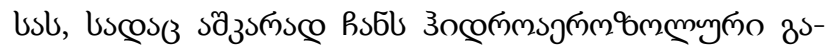

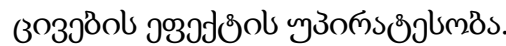

\section{cosb $336 s$}

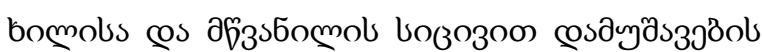

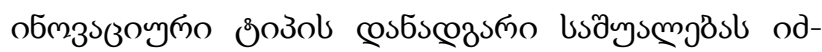

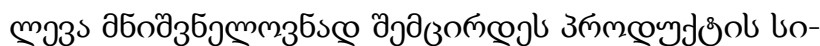

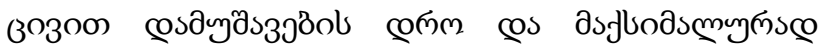

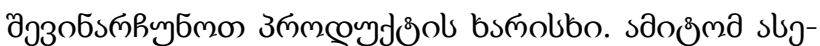

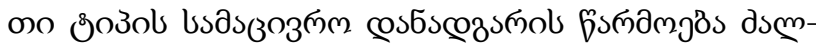

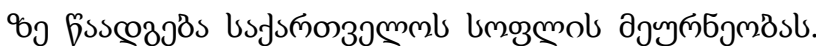

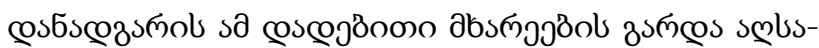

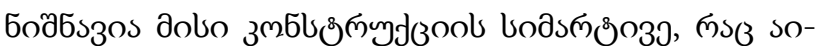

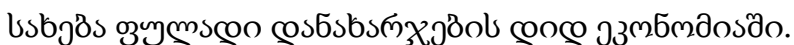




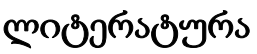

1. Megrelidze T., Japaridze Z., Suladze S., Gugulashvili G., Goletiani G., Tepnadze A., Kvirikashvili G., Omiadze Z. Refrigerator machines (Piston compressors). "Teqnikuri Universiteti". Tbilisi. 2009, 52-53 pp. (in Georgian).

2. Megrelidze T., Sadagashvili E., Beruashvili G., Gugulashvili G. Study of the optimal working regimes of refrigerator machines with difficult cycle. "Teqnikuri Universiteti". \#2 (480). Tbilisi. 2011, 91-96 pp. (in Georgian).

3. System trouble shooting measuring instruments. Danfoss A/S (RC-SM/MWA), 09-2002. (in Russian).

4. Honeywell refrigerants. Honeywell International Inc. 2006.

UDC 620.16

SCOPUS CODE 2209

DOI: https://doi.org/10.36073/1512-0996-2019-3-54-60

\section{Hydroelectric technology of refrigeration processing of fruits and greens}

\author{
Gia Goletiani \\ Department of Food Industry, Georgian Technical University, 68 a M. Kostava str, 0160 \\ Tbilisi, Georgia \\ E-mail: gia_goletiani@yahoo.com \\ Tamaz Isakadze \\ Department of Food Industry, Georgian Technical University, 68 a M. Kostava str, 0160 \\ Tbilisi, Georgia \\ E-mail: tamazisakadze@gmail.com \\ Givi Gugulashvili \\ Department of Food Industry, Georgian Technical University, 68 a M. Kostava str, 0160 \\ Tbilisi, Georgia \\ E-mail: Givi.Gugulashvili@gmail.com
}

\section{Reviewers:}

Z. Japaridze, Professor, Faculty of Transportation and Mechanical Engineering, GTU

E-mail: zurabjaparidze@yahoo.com

S. Suladze, Doctor of Technical Sciences, Director of LTD "Georgian Refrigerant Recovery and Recycling Center"

E-mail: sulkhansuladze@gmail.com

Abstract. A universal method of preserving the quality of greens and fruits is hydrolic cold processing technology. This method involves creating of the required temperature and maintaining the necessary relative humidity for the product. Unlike the cooling of the product, innovative technology with use of classical technology of storage of fruits and greens, includes rode holding moisture using special devices into smaller pieces. Compounding devices are tangential-type nozzles that can form a region of water mist in a fruit or greens storage.

Since fruits and greens are considered as living products, the innovative refrigeration equipment design is based on supply and exhaust ventilation, which allows the environment to be filled with clean air. The production of this type of equipment, in contrast to the factory facilities, is distinguished by great money savings.

Key words: Capillary tube; compressor; condenser; oil separator; ventilator. 


\section{UDC 620.16}

SCOPUS CODE 2209

DOI: https://doi.org/10.36073/1512-0996-2019-3-54-60

\section{Гидроаэрозольная технология холодильной обработки фруктов и зелени}

Гия Голетиани

Тамаз Исакадзе

Гиви Гугулашвили
Департамент пищевой индустрии, Грузинский технический университет, Грузия, 0160, Тбилиси, ул. М. Костава, 68

E-mail: gia_goletiani@yahoo.com

Департамент пищевой индустрии, Грузинский технический университет, Грузия, 0160, Тбилиси, ул. М. Костава, 68

E-mail: tamazisakadze@gmail.com

Департамент пищевой индустрии, Грузинский технический университет, Грузия, 0160, Тбилиси, ул. М. Костава, 68

E-mail: Givi.Gugulashvili@gmail.com

\section{Рецензенты:}

3. Джапаридзе, профессор факультета транспорта и машиностроения ГТУ

E-mail: zurabjaparidze@yahoo.com

С. Суладзе, директор ООО «Центра сбора и рециклирования хладоагентов Грузии», доктор технических наук E-mail: sulkhansuladze@gmail.com

Аннотащия. Универсальным методом сохранения качества зелени и фруктов является гидроаэрозольная технология холодной обработки. Этот метод включает создание необходимой температуры и поддержание необходимой относительной влажности для продукта. Инновационная технология в отличие от охлаждения продукта, с использованием классической технологии хранения фруктов и зелени, включает поддержку влажности с помощью специальных увлажнительных устройств на более мелкие части. Увлажнительными устройствами являются форсунки тангенциального типа, которые могут образовывать в хранилище зелени или овощей область водяного тумана.

Поскольку фрукты и зелень считаются живыми продуктами, схема инновационного холодильного оборудования снабжена приточно-вытяжной вентиляцией, которая позволяет заполнять окружающую среду чистым воздухом. Производство такого типа оборудований в отличие от заводских устройств, отличается большой экономией денежных средств.

Ключевые слова: вентилятор; капиллярная труба; компрессор; конденсатор, маслоотделитель.

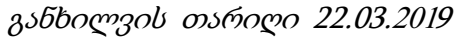

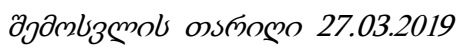

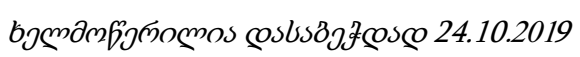


UDC 621.51:621.56/.57

SCOPUS CODE 2209

DOI: https://doi.org/10.36073/1512-0996-2019-3-61-67

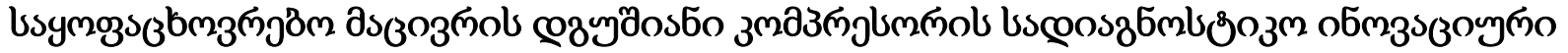

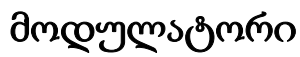

zos zmmjoosto

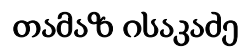

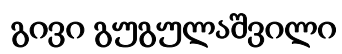

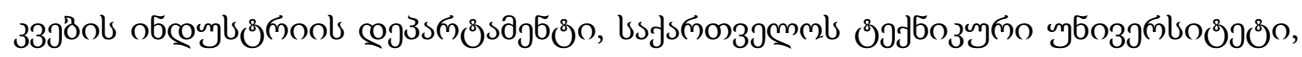

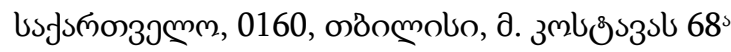

E-mail: gia_goletiani@yahoo.com

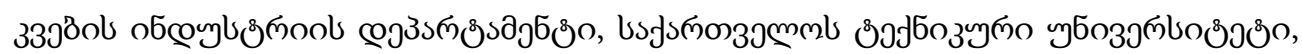

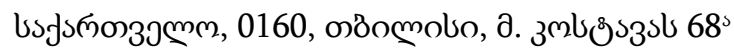

E-mail: tamazisakadze@gmail.com

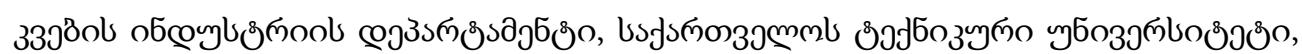

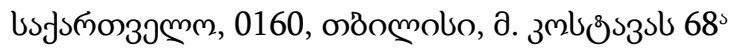

E-mail: Givi.Gugulashvili@gmail.com

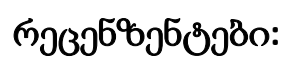

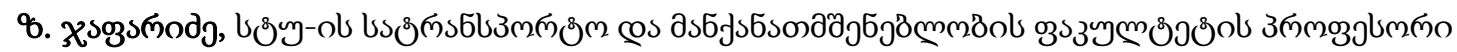

E-mail: zurabjaparidze@yahoo.com

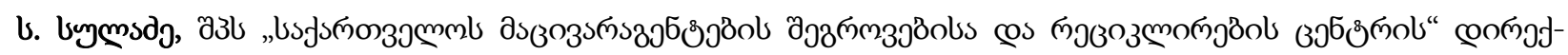

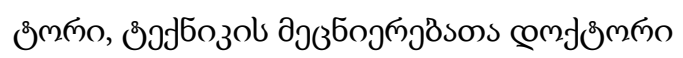

E-mail: sulkhansuladze@gmail.com

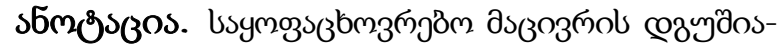

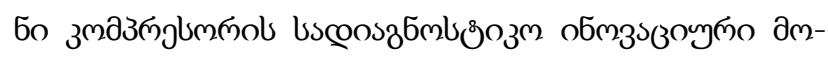

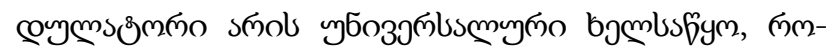

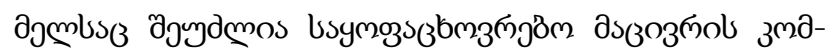

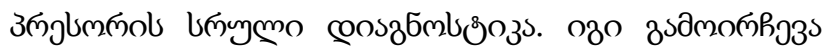

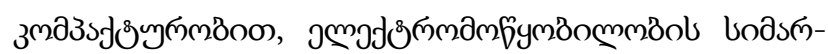

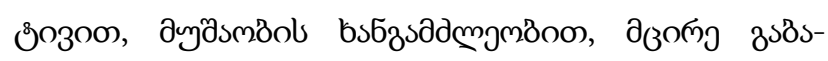

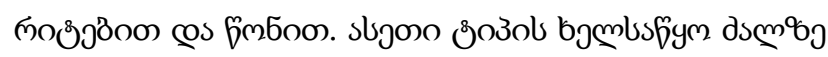

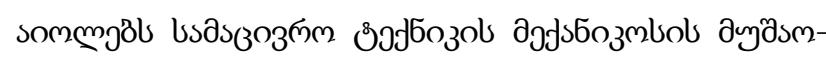

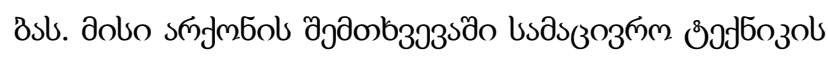

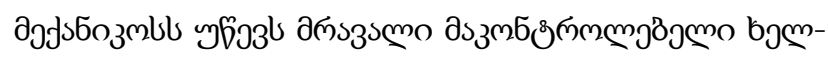

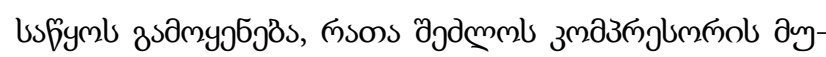

\section{ISSN 1512-0996}

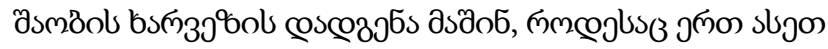

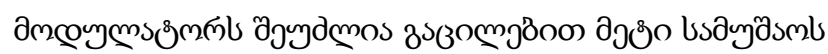

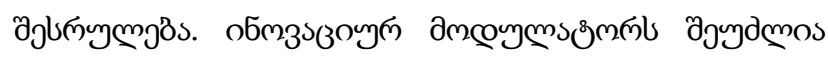

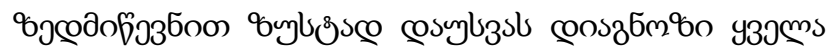

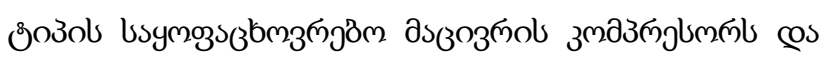

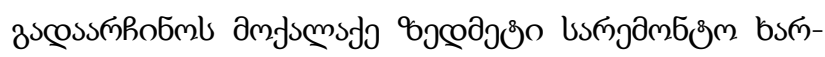

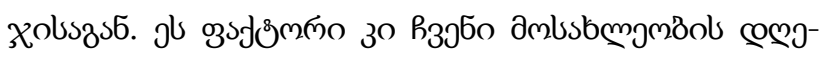

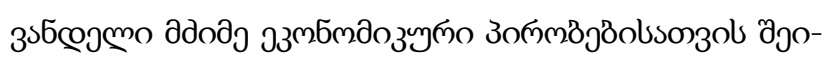

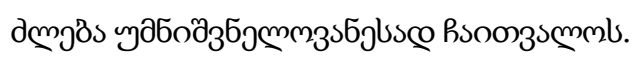

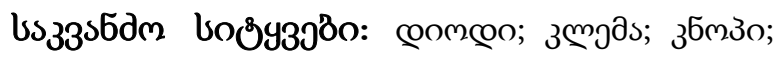

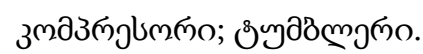

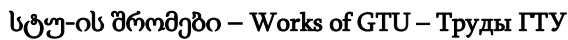
№3 (513), 2019 


\section{gанsзsемо}

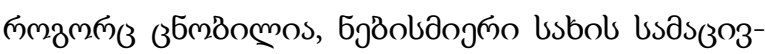

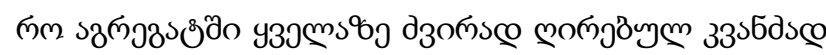

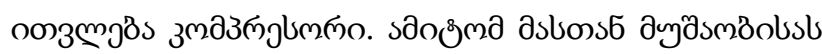

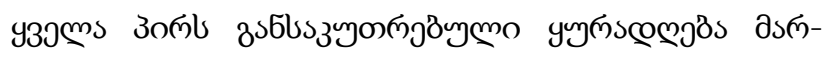

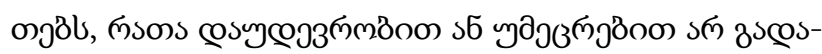

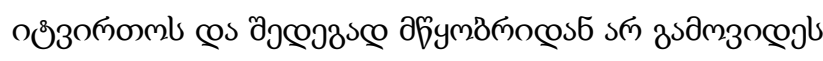

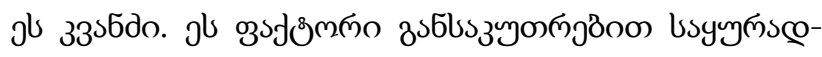

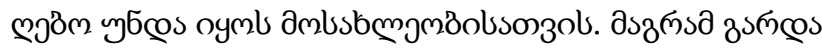

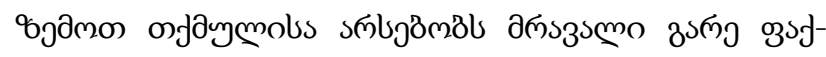

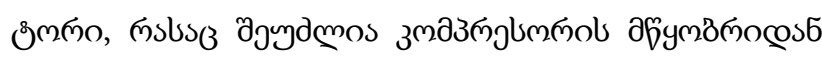

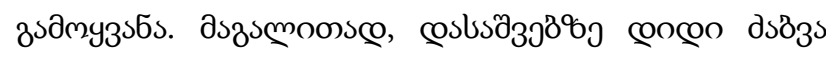

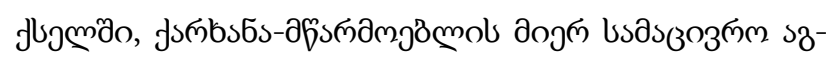

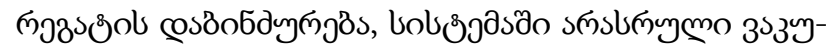

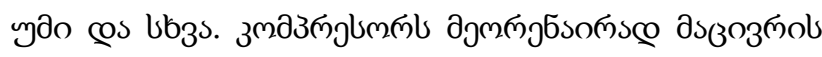

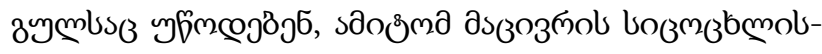

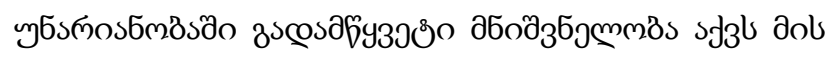

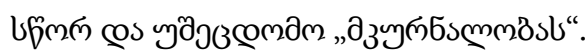

\section{doпоonscen 6sformo}

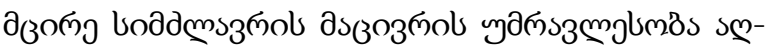

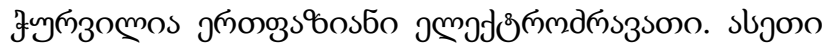

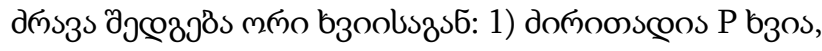

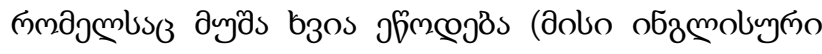

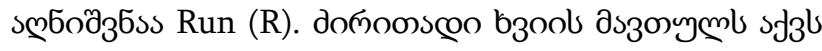

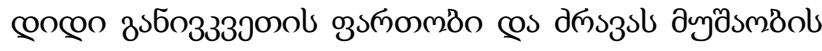

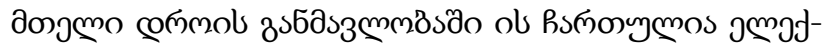

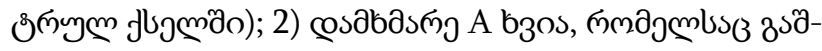

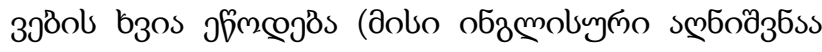

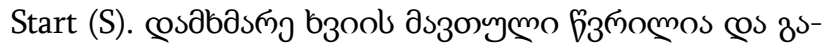

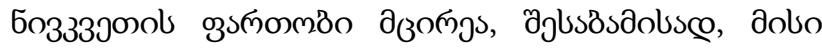
бобзмm8s goncos).

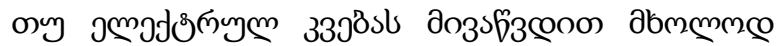

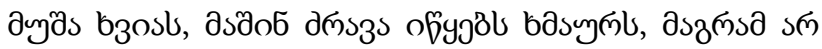

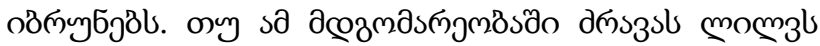

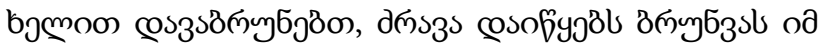

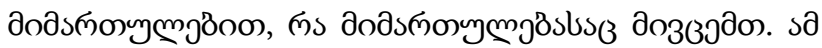

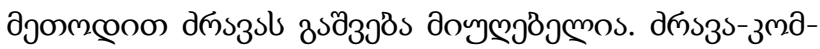

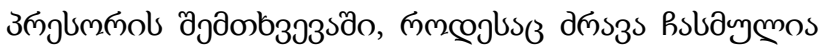
moom

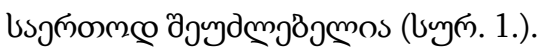

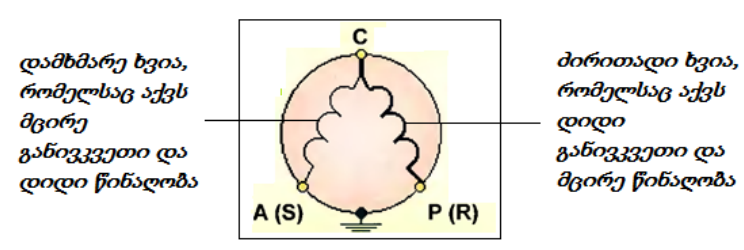

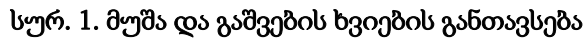

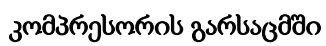

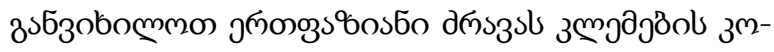

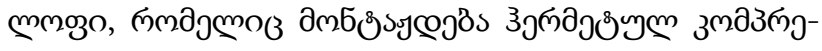

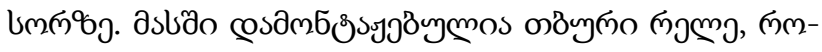

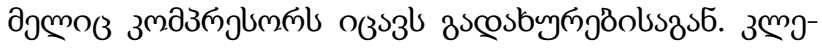

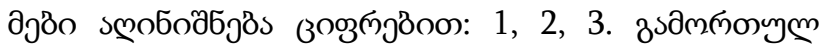

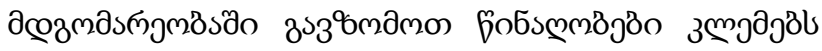

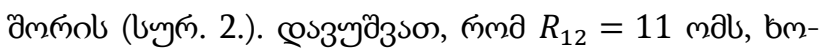
мм $R_{13}=3$ маl. дsəoб $R_{32}=14$ мals.

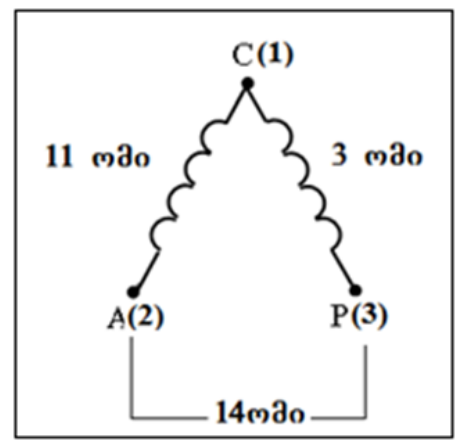

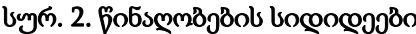

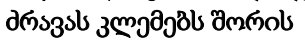




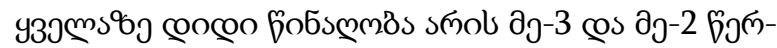

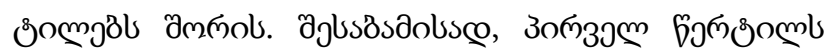

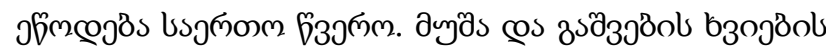

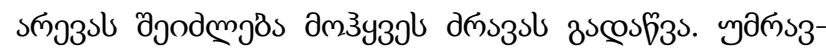

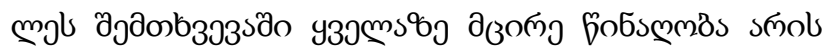

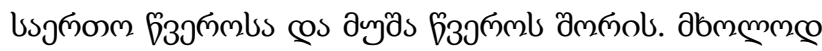

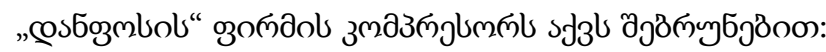

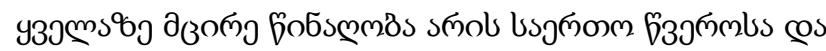

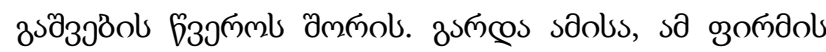

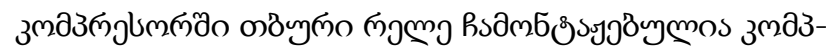

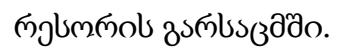

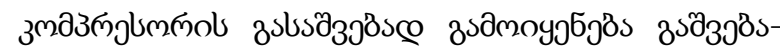

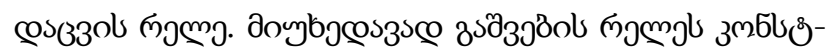

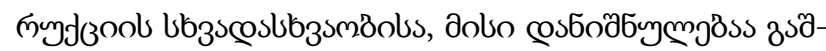

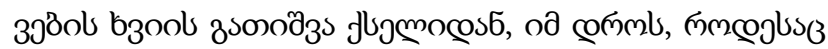

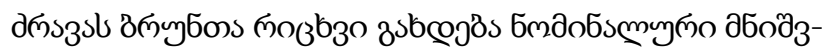

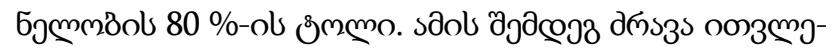

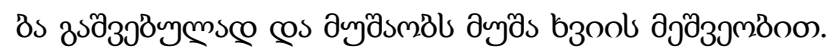

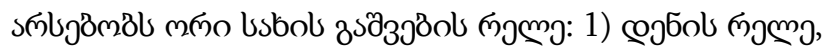

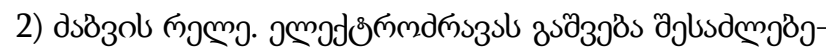

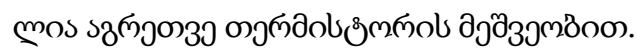

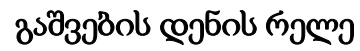

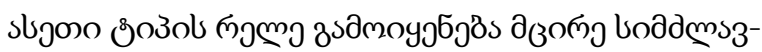

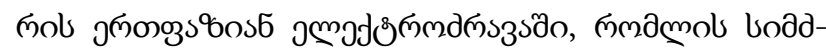

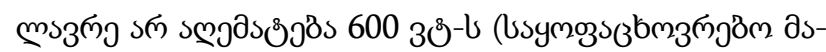

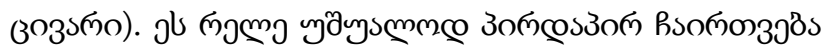

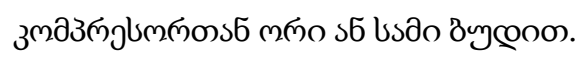

\begin{tabular}{|c|c|c|}
\hline $\mathrm{P} / \mathrm{M}^{\wedge}$ & ayzs (Main) & $->$ ayas b3กs \\
\hline$A / S$->. & (START) & 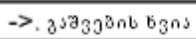 \\
\hline$L->$ & (Line) & $\Rightarrow .330^{3} \cap 6 \mathrm{~g} 38 \mathrm{~s}$ \\
\hline
\end{tabular}

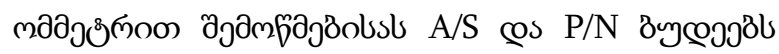

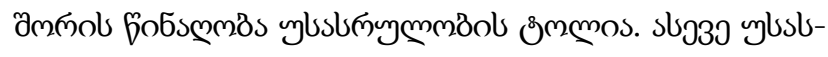

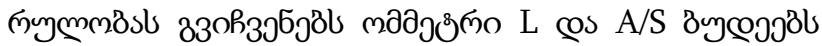

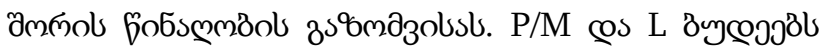

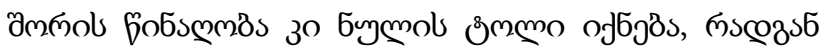

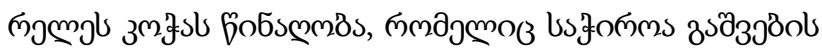

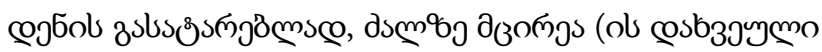

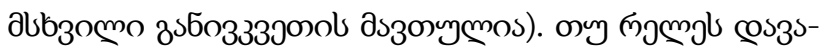

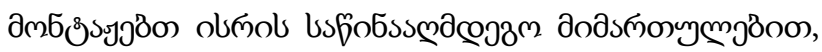

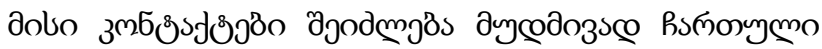

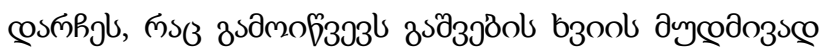

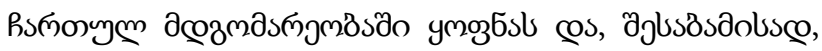

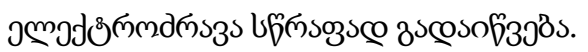

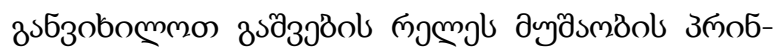

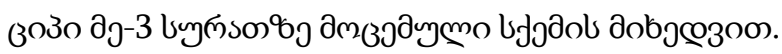

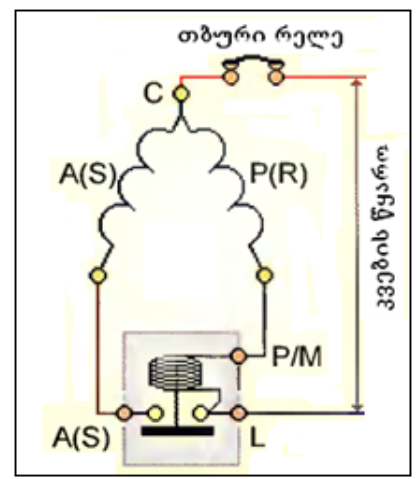

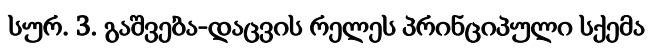

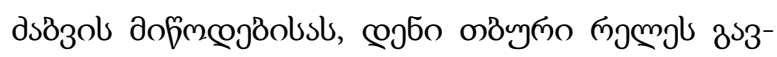

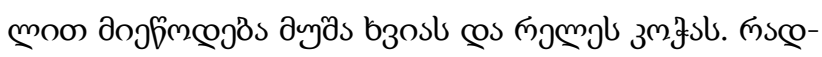

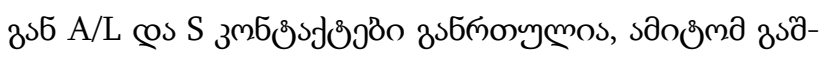

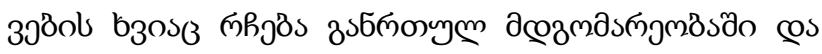

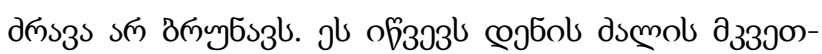

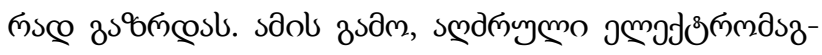

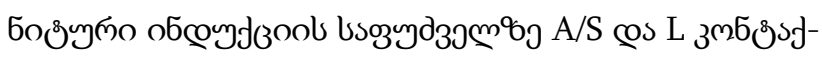

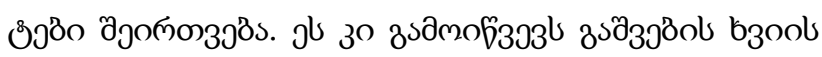

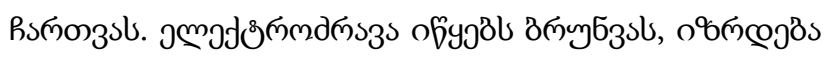

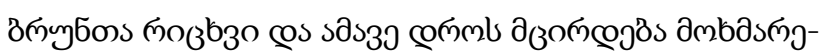




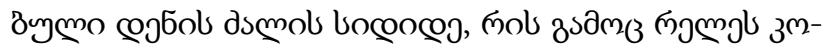

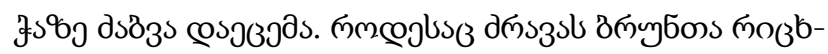

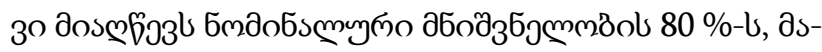

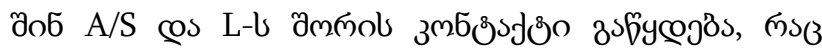

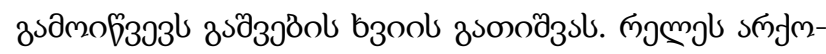

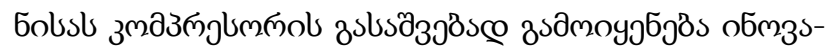

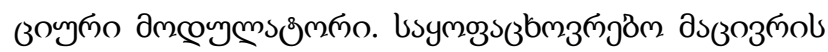

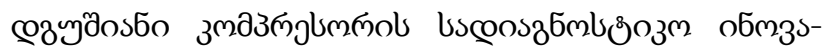

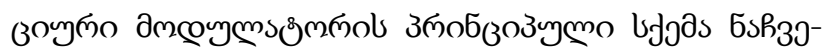

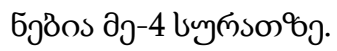

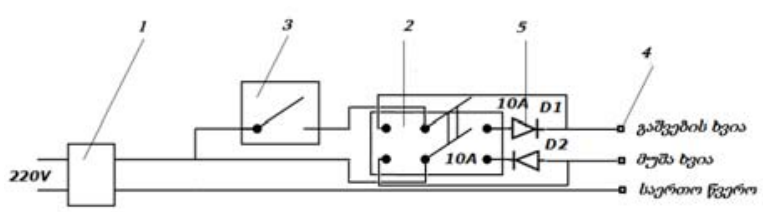

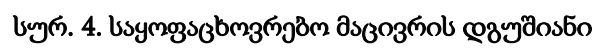

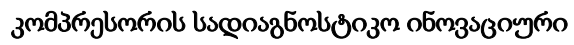

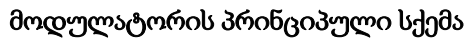

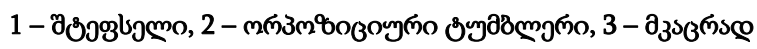

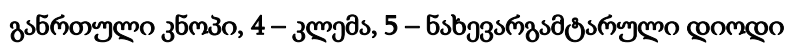

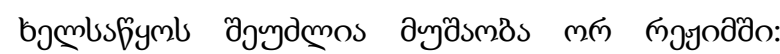

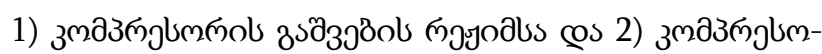

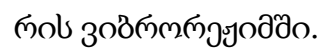

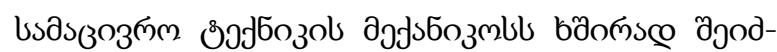

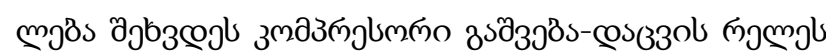

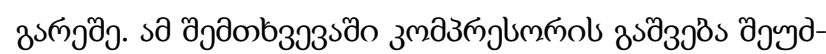

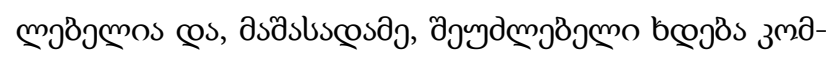

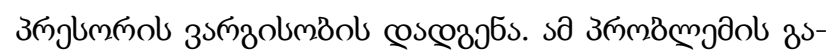

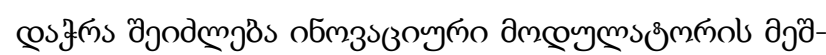

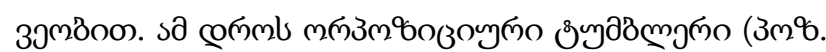

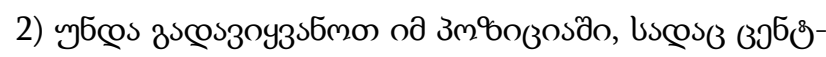

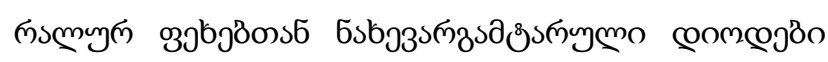

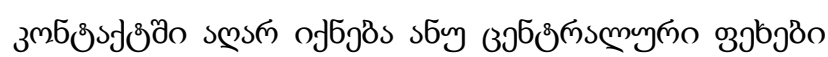

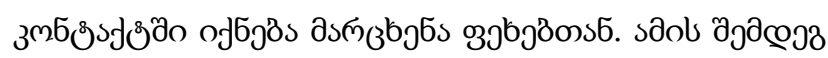

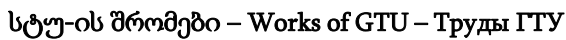
№3 (513), 2019

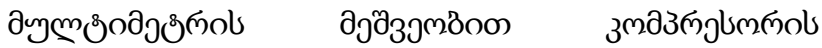

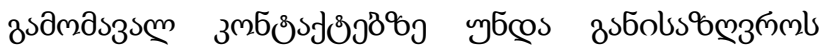

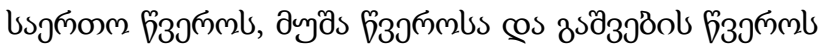

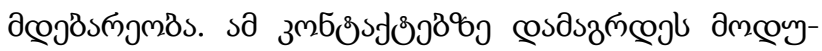

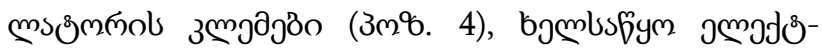

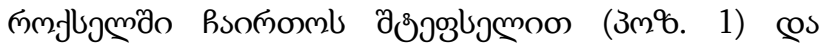

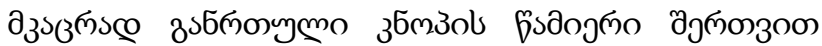

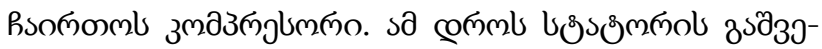

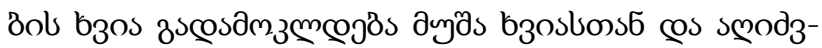

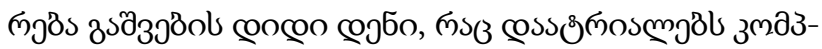

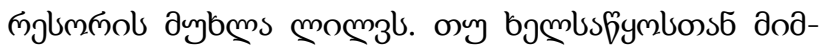

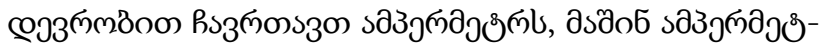

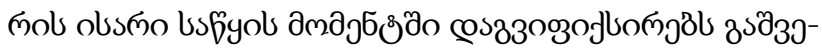

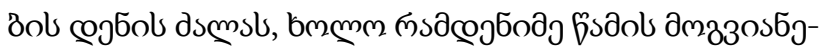

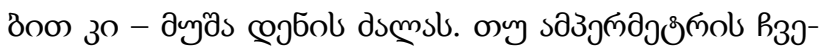

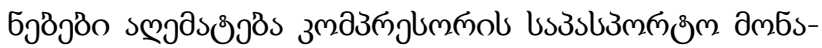

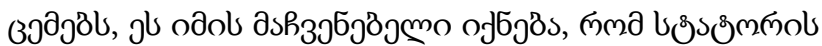

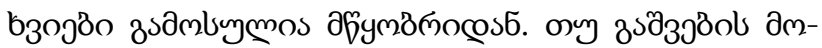

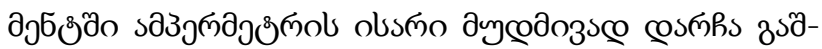

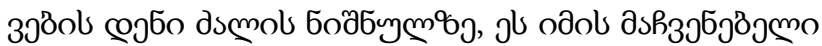

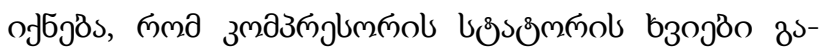

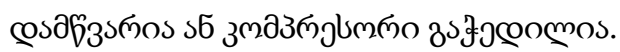

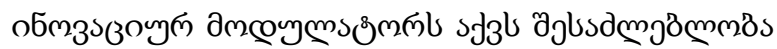

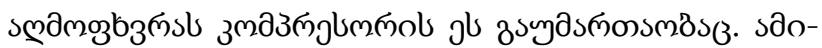

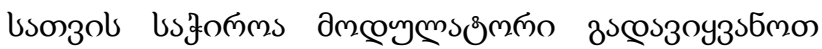

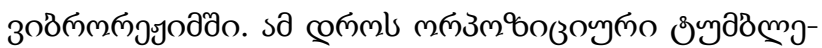

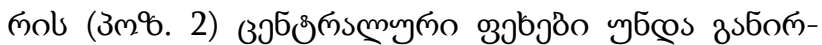

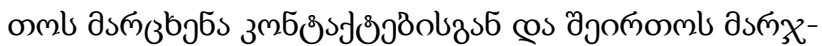

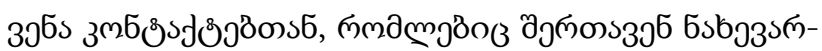

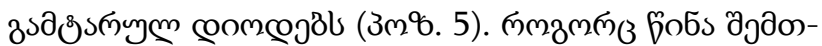

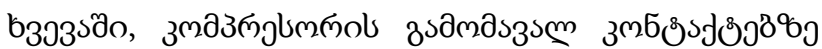

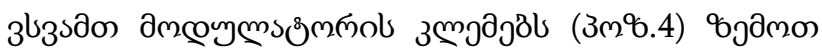

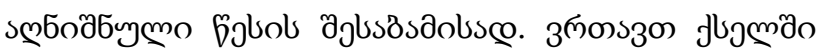




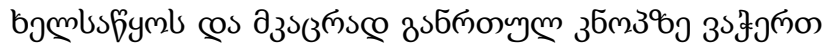

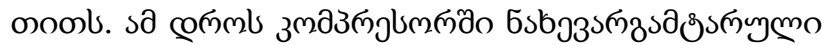

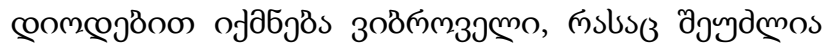

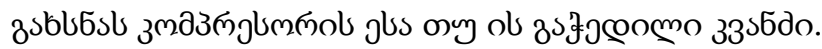

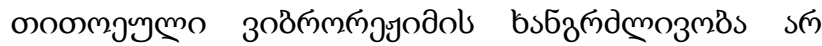

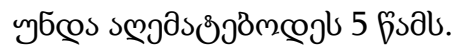

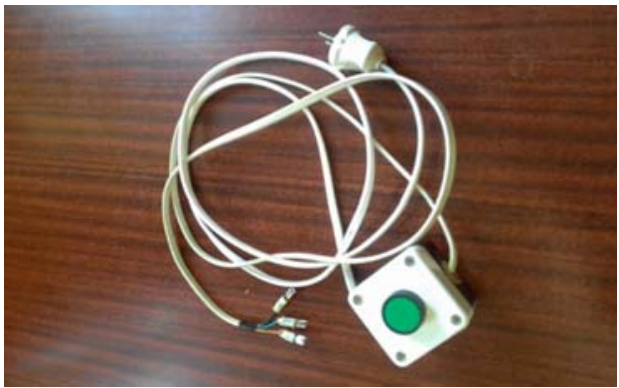

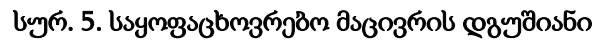

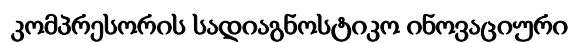

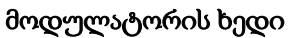

\section{cosb $33^{6 s}$}

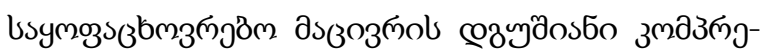

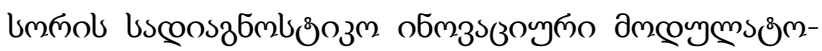

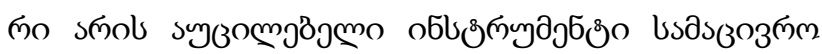

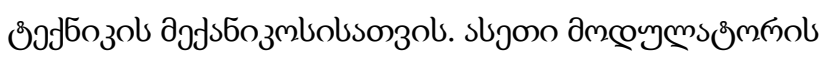

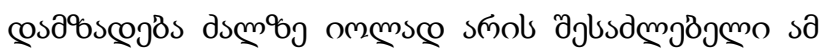

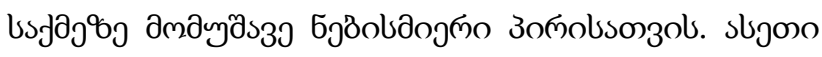

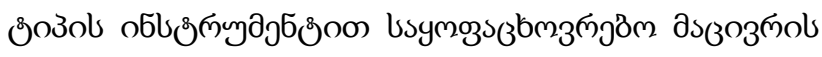

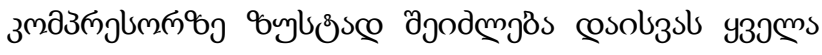

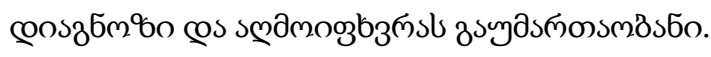

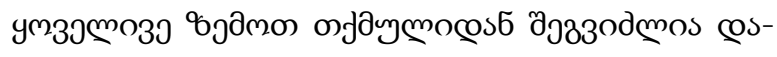

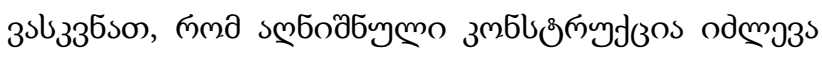

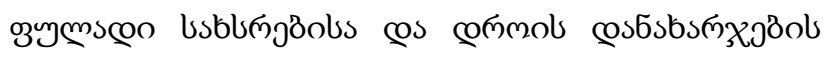

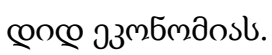

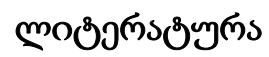

1. Megrelidze T., Japaridze Z., Suladze S., Gugulashvili G., Goletiani G., Tepnadze A., Kvirikashvili G., Omiadze Z. Refrigerator machines (Piston compressors). "Teqnikuri Universiteti”. Tbilisi. 2009, 52-53 pp. (in Georgian).

2. Megrelidze T., Sadagashvili E., Beruashvili G., Gugulashvili G. Study of the optimal working regimes of refrigerator machines with difficult cycle. "Teqnikuri Universiteti". \#2 (480). Tbilisi. 2011, 91-96 pp. (in Georgian).

3. System trouble shooting measuring instruments. Danfoss A/S (RC-SM/MWA), 09-2002. (in Russian).

4. Honeywell refrigerants. Honeywell International Inc. 2006.

ISSN 1512-0996

www.shromebi.gtu.ge 


\title{
UDC 6
}

SCOPUS CODE 2209

DOI: HTTPS://DOI.ORG/10.36073/1512-0996-2019-3-61-67

\section{Diagnostic innovative modulator for piston compressors of household refrigerators}

\author{
Gia Goletiani Department of Food Industry, Georgian Technical University, 68 a M. Kostava str, 0160 \\ Tbilisi, Georgia \\ E-mail: gia_goletiani@yahoo.com \\ Tamaz Isakadze Department of Food Industry, Georgian Technical University, 68 a M. Kostava str, 0160 \\ Tbilisi, Georgia \\ E-mail: tamazisakadze@gmail.com \\ Givi Gugulashvili Department of Food Industry, Georgian Technical University, 68 a M. Kostava str, 0160 \\ Tbilisi, Georgia \\ E-mail: Givi.Gugulashvili@gmail.com
}

\section{Reviewers:}

Z. Japaridze, Professor, Faculty of Transportation and Mechanical Engineering, GTU

E-mail: zurabjaparidze@yahoo.com

S. Suladze, Doctor of Technical Sciences, Director of LTD "Georgian Refrigerant Recovery and Recycling Center"

E-mail: sulkhansuladze@gmail.com

\begin{abstract}
The diagnostic innovative modulator for piston compressors of household refrigerators is a universal tool capable for complete diagnosis of compressors. It is distinguished by its compactness, simplicity of the electric device, durability, small dimensions and weight of work. This type of device facilitates the work of the refrigerator. In case of non-availability, the refrigerator has to use a number of controlling devices to determine the shortcomings of the compressor work, while one such modulator can perform much more work than all abovementioned. An innovative modulator can accurately diagnose all types of household refrigerators compressors and save citizens from excessive repair costs. This can be considered as critically important factor for severe economic conditions of today.
\end{abstract}

Key words: Compressor; connection terminal; diode; knop; tumbler. 


\section{UDC 6}

SCOPUS CODE 2209

DOI: HTTPS://DOI.ORG/10.36073/1512-0996-2019-3-61-67

\section{Диагностический инновационный модулятор для поршневых компрессоров бытовых холодильников}

Гия Голетиани

Тамаз Исакадзе

Гиви Гугулашвили
Департамент пищевой индустрии, Грузинский технический университет, Грузия, 0160, Тбилиси, ул. М. Костава, 68

E-mail: gia_goletiani@yahoo.com

Департамент пищевой индустрии, Грузинский технический университет, Грузия, 0160, Тбилиси, ул. М. Костава, 68

E-mail: tamazisakadze@gmail.com

Департамент пищевой индустрии, Грузинский технический университет, Грузия, 0160, Тбилиси, ул. М. Костава, 68

E-mail: Givi.Gugulashvili@gmail.com

\section{Рецензенты:}

3. Джаппаридзе, профессор факультета транспорта и машиностроения ГТУ.

E-mail: zurabjaparidze@yahoo.com

С. Суладзе, директор ООО «Центра сбора и рециклирования хладоагентов Грузии», доктор технических наук

E-mail: sulkhansuladze@gmail.com

Аннотация. Диагностический инновационный модулятор поршневых компрессоров бытовых холодильников-универсальный инструмент, способный полностью диагностировать компрессоры. Он отличается компактностью, простотой электрического устройства, долговечностью, малыми габаритами и весом. Этот тип устройства очень облегчает работу холодильщика. В случае отсутствия такого инструмента, холодильщик должен использовать несколько устройств для определения недостатков работы компрессора, в то время как один такой модулятор может выполнять намного больше работы. Инновационный модулятор может точно диагностировать все типы компрессоров бытовых холодильников и спасать граждан от чрезмерных затрат на ремонт. Этот фактор можно рассматривать как решающую черту для суровых экономических условий сегодняшнего дня.

Ключевые слова: диод; клемма; кнопка; компрессор; тумблер.

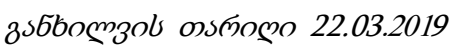

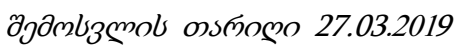

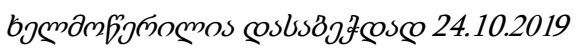


UDC 65.012.122:658.284

SCOPUS CODE 2209

DOI: https://doi.org/10.36073/1512-0996-2019-3-68-77

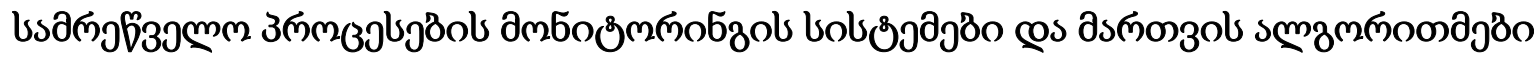

usलmmaว мбоsбo

os ambsðुomo

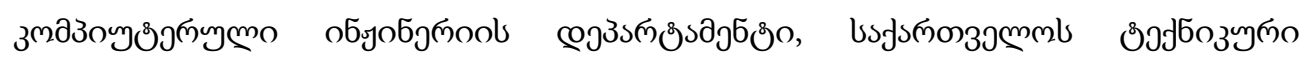

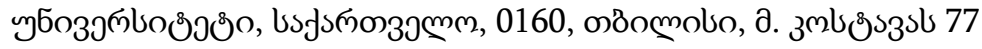

E-mail: s.oniani@gtu.ge

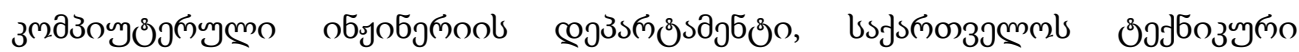

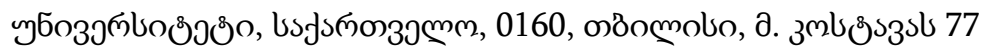

E-mail: i_mosashvili@gtu.ge

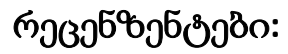

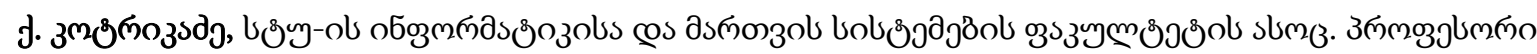

E-mail: ketino27@gmail.com

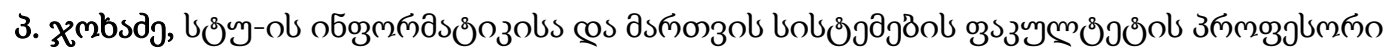

E-mail: paata_j@yahoo.com

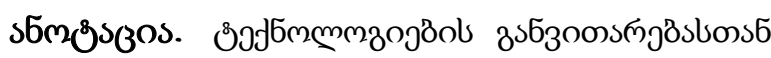

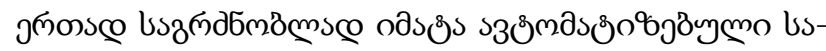

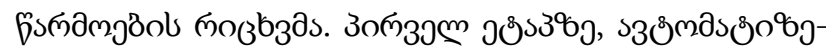

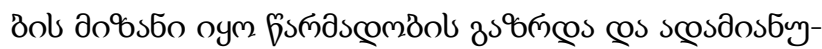

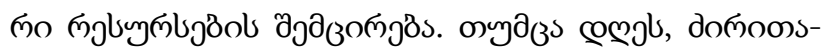

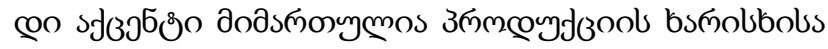

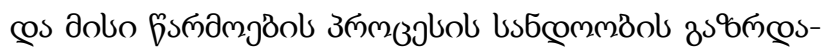

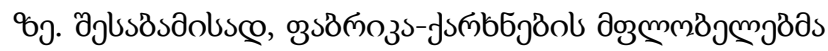

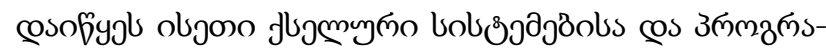

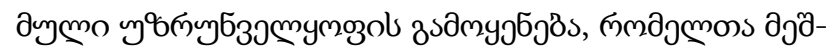

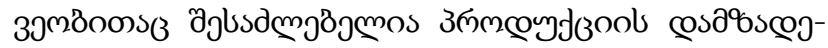

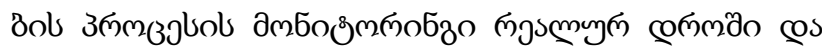

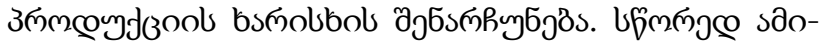

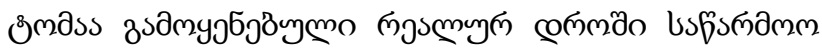

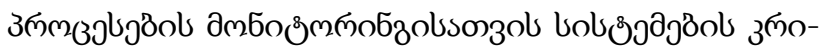

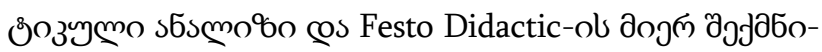

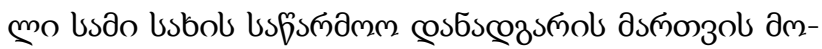

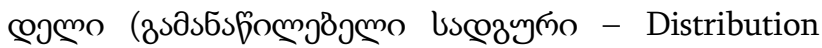

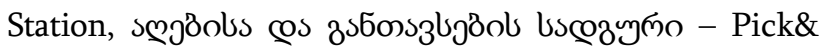
Place Station, cosbśnolbjöol uscayno - Sorting Station).

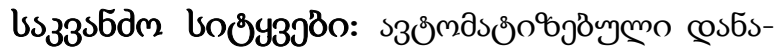

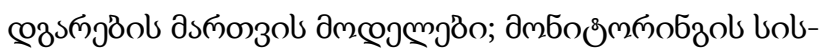

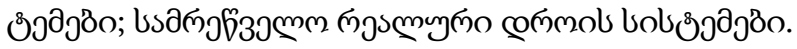




\section{פjuszsmo}

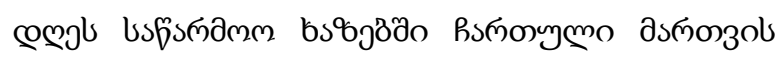

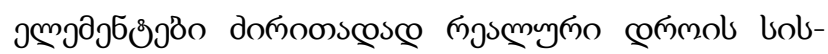

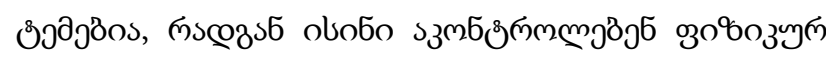

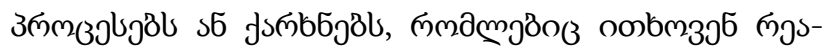

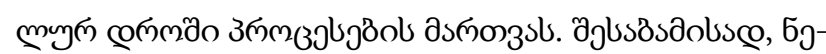

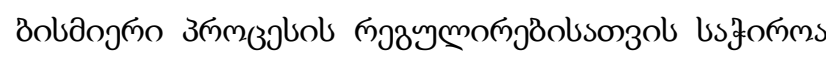

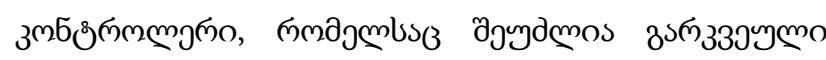

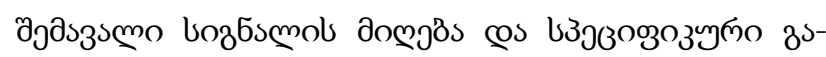

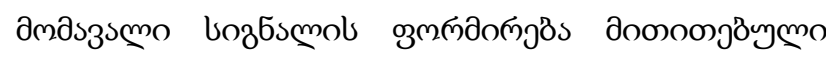

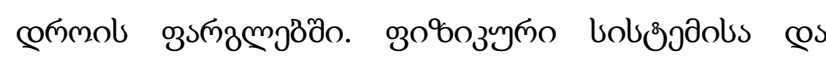

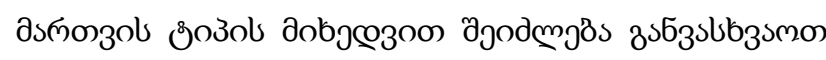

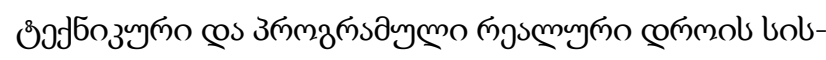

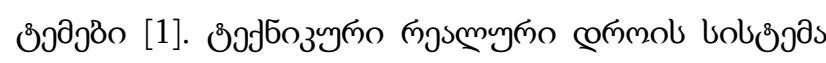

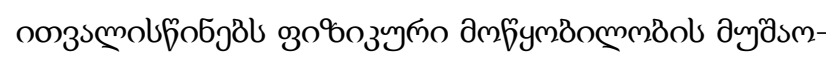

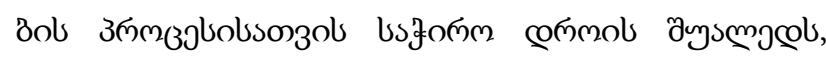

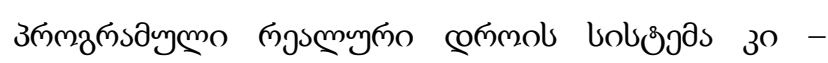

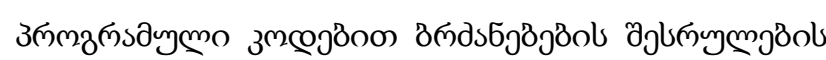
bsбъпддмозмыsl.

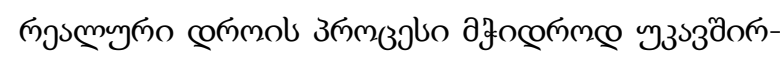

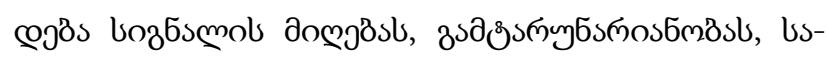

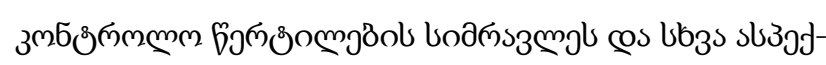

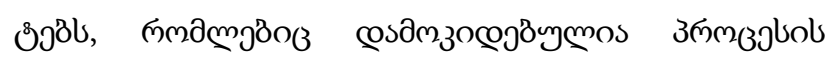

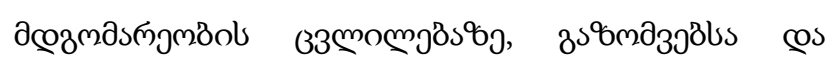

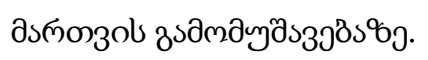

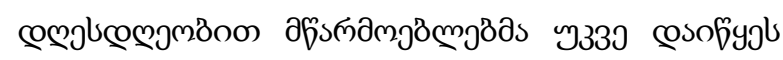

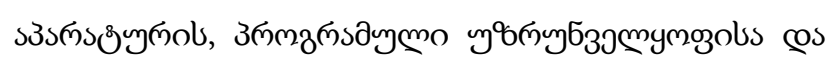

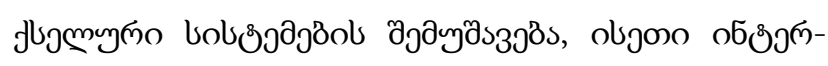
бృภbs

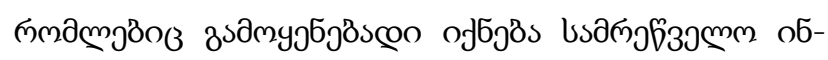

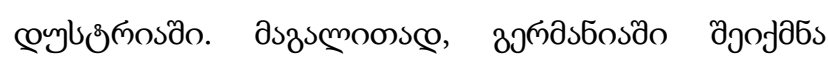

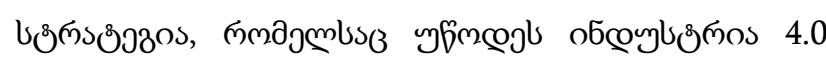

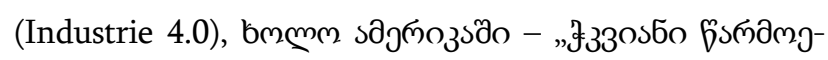

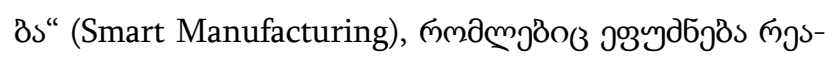

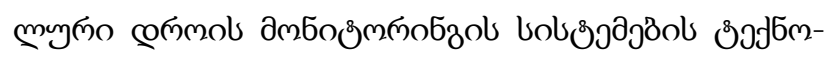

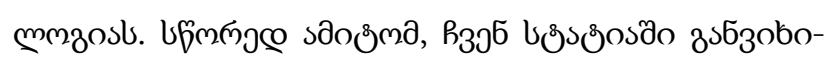

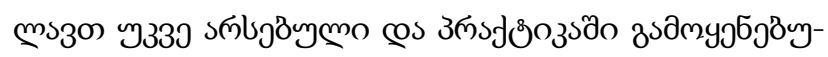

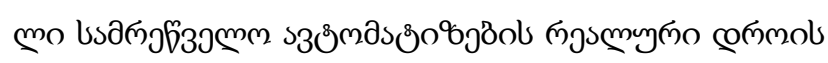

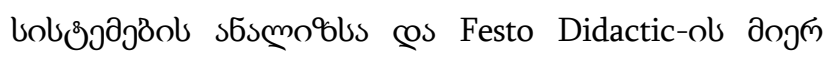

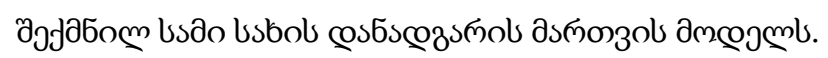

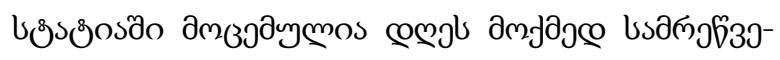

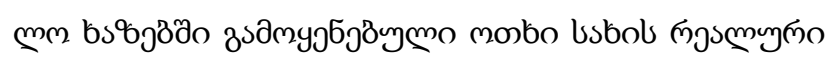

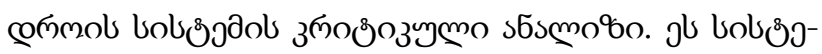

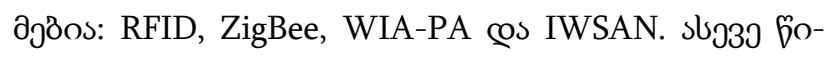

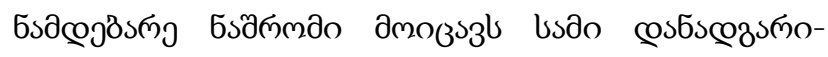

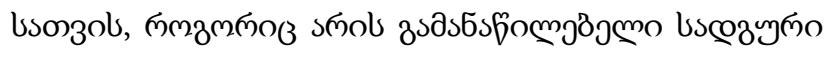

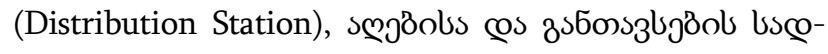

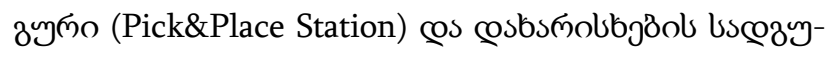

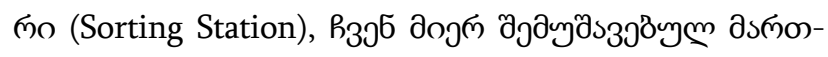
зоь sмздмпопдја̋.

\section{donnoosseo 5sfomo}

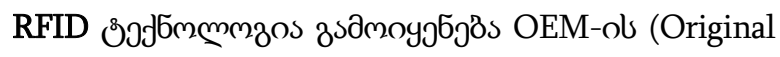

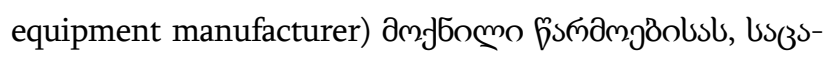

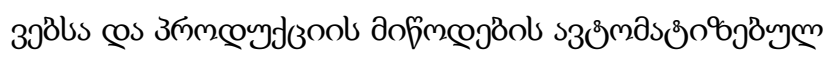

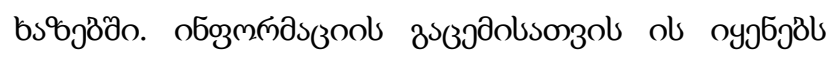

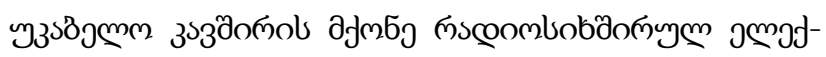

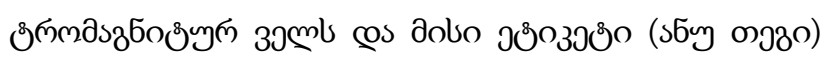

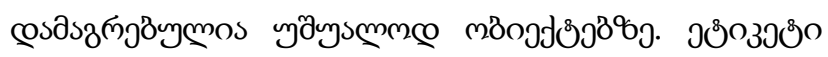

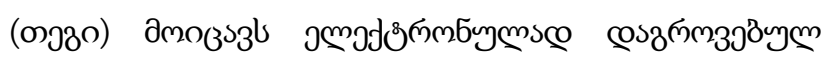

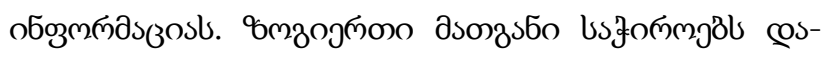

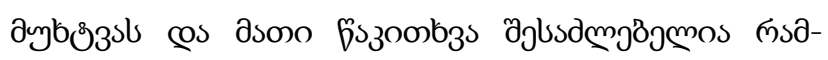

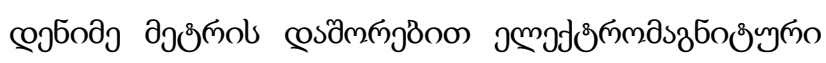
обœу

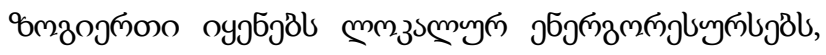

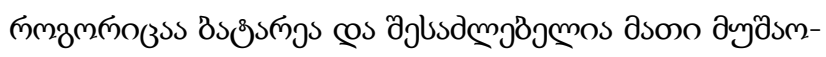




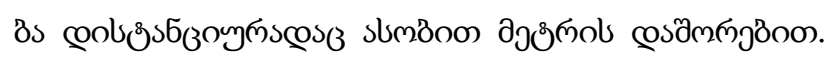

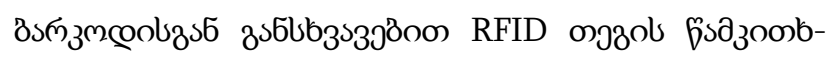

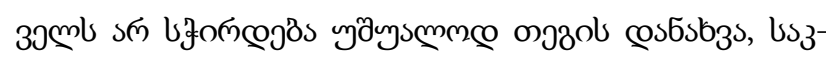

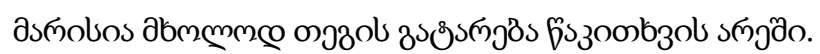

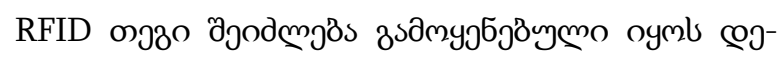

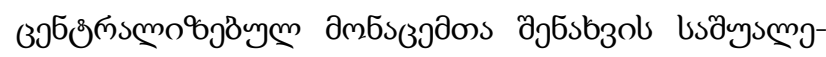

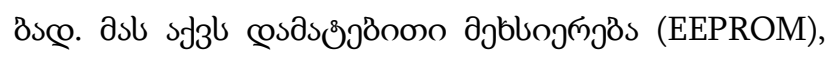

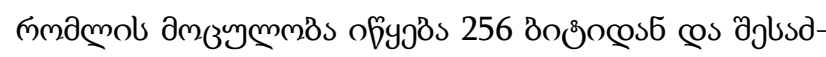

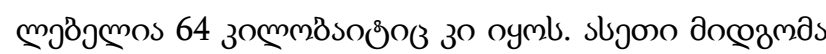

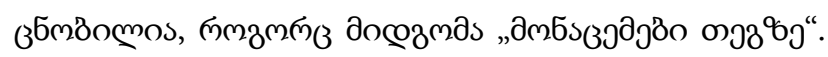

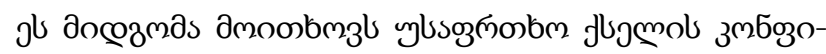

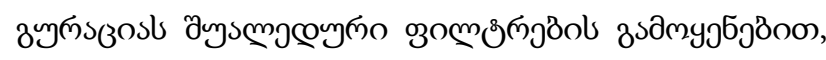

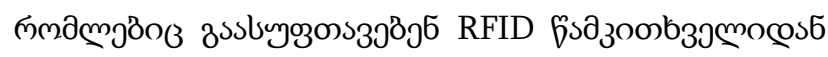

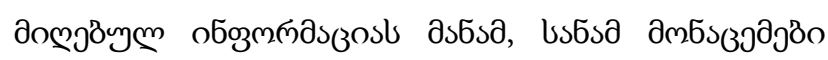

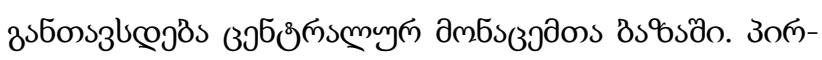

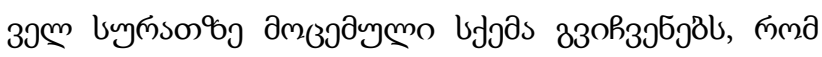

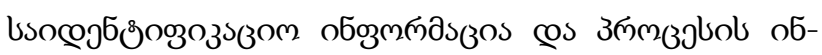

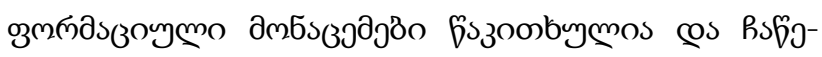

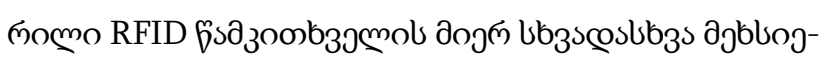

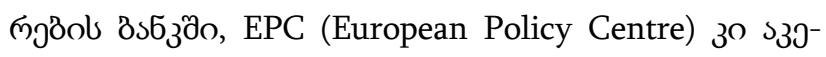

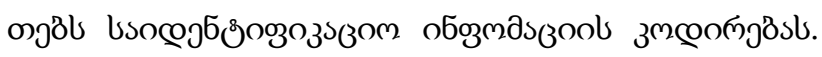

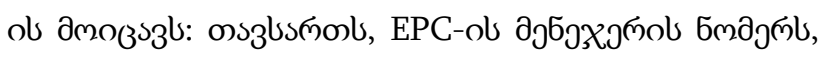

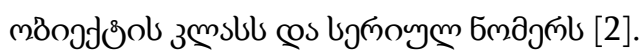

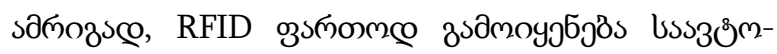

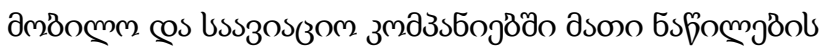

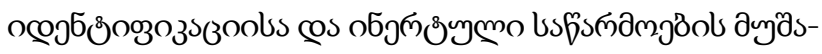

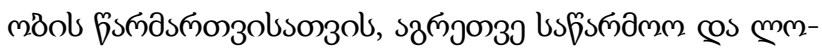

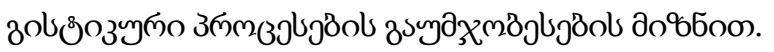

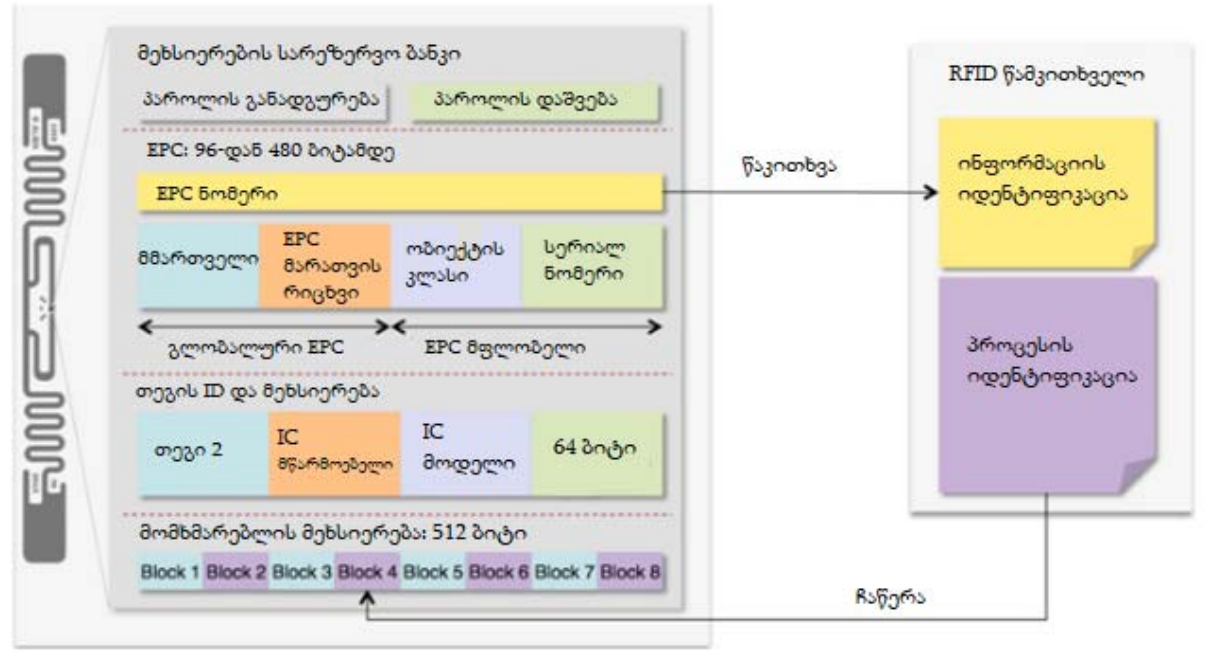

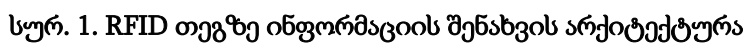

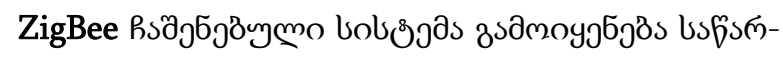

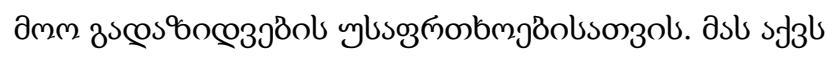

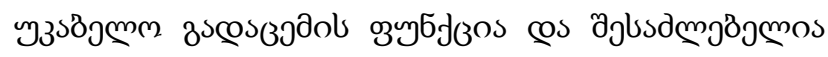

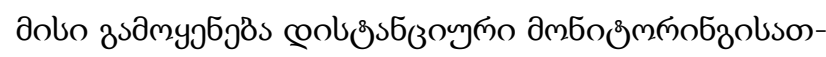

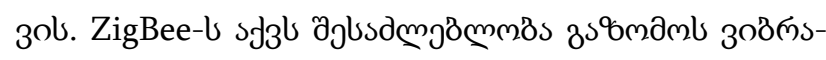

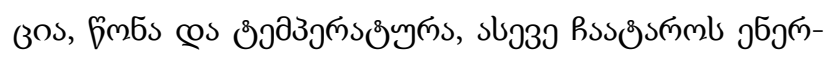

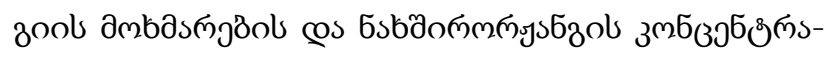

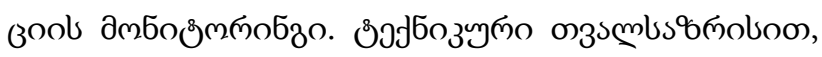

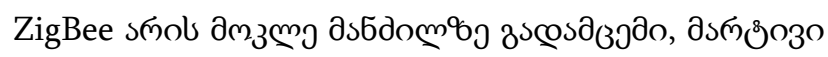

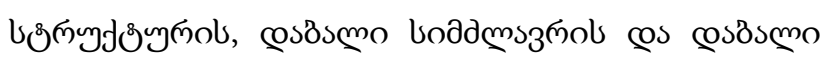

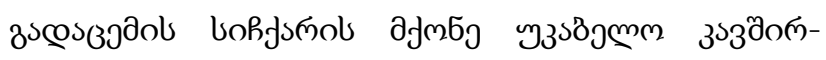

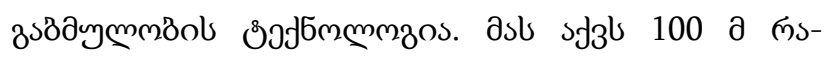




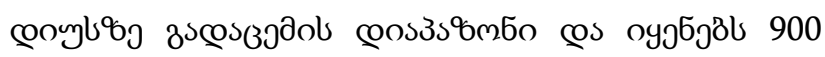

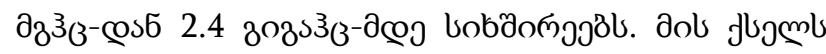

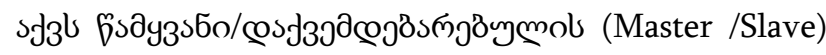

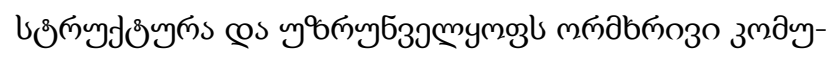

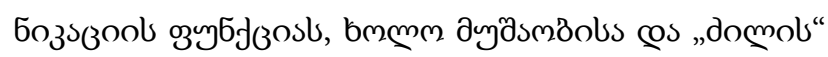

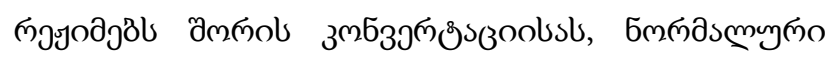

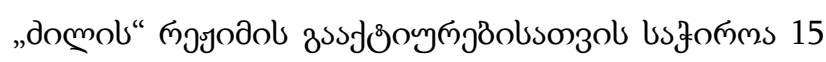

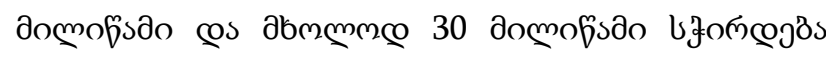

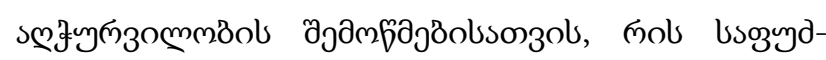

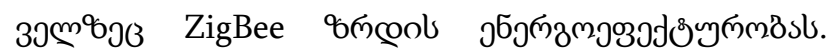

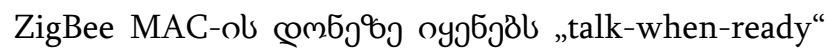

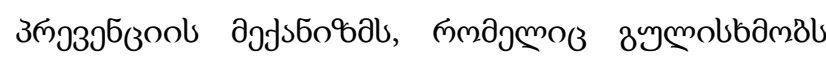

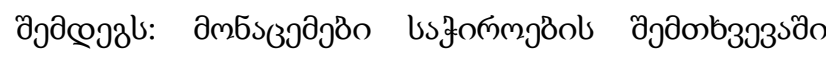

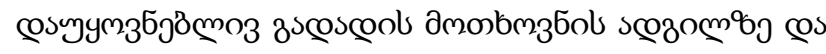

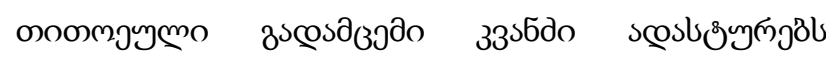

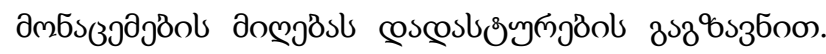

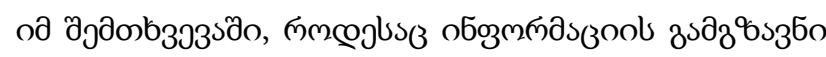

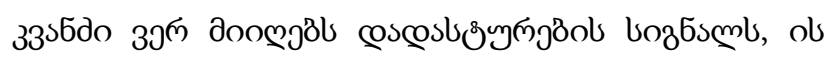

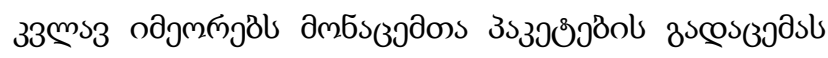

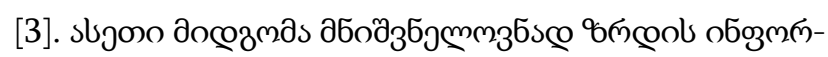

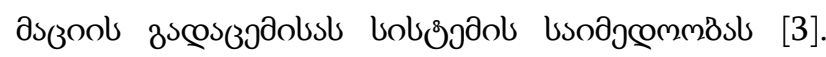

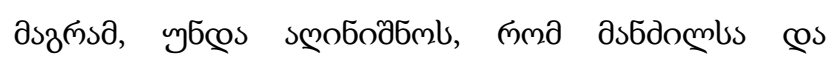

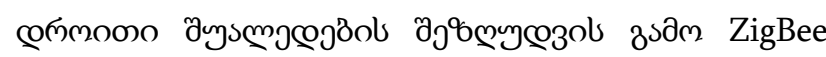

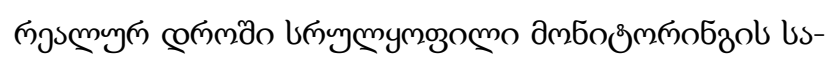

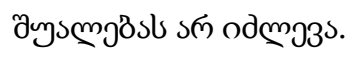

WIA-PA (Wireless Networks for Industrial Automa-

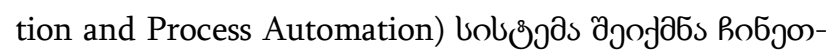

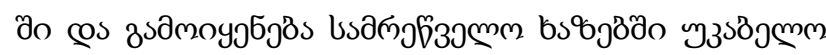

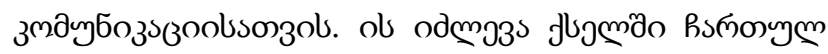

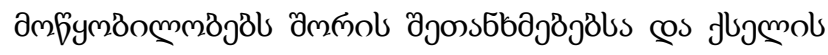

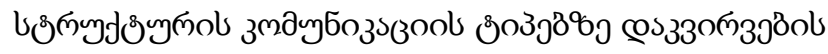

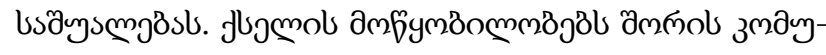

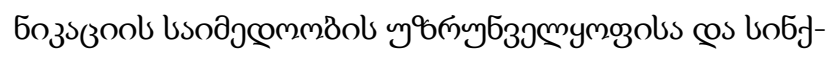

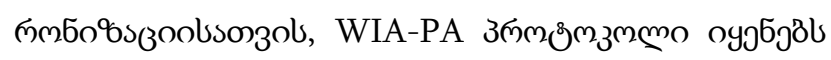

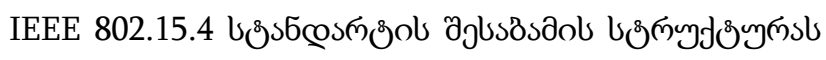

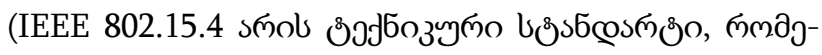

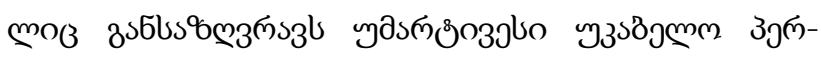

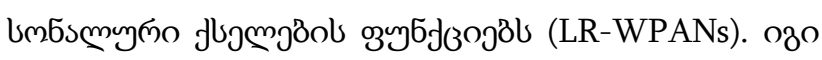

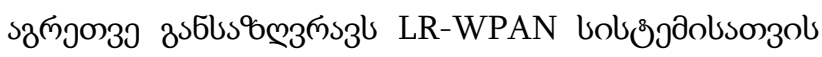

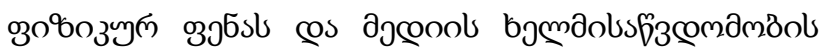

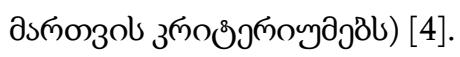

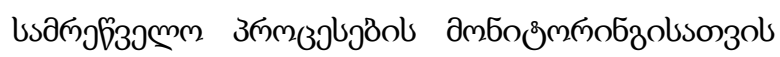
WIA-PA uobతి

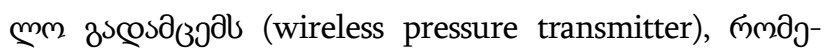

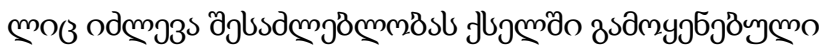

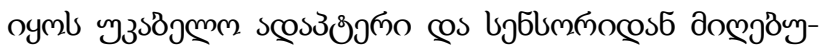

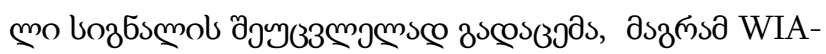

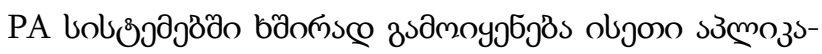

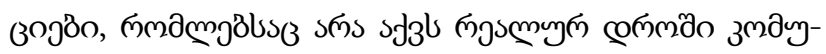

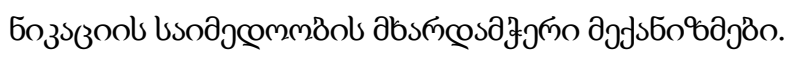

иsдп

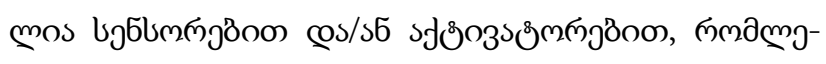

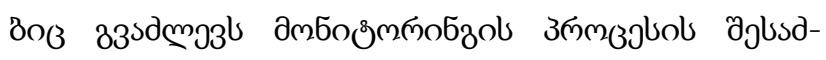

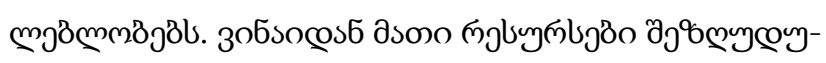

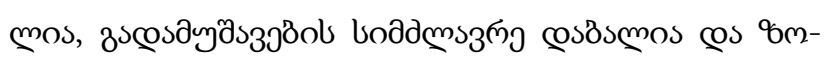

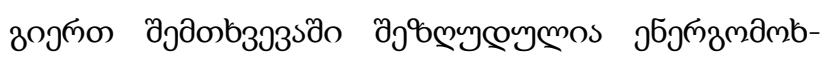

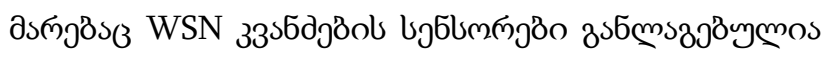

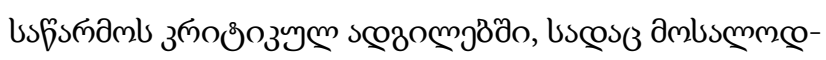

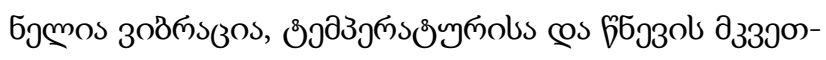

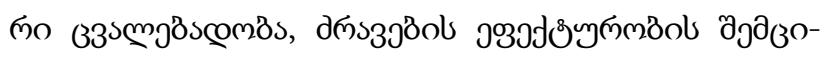

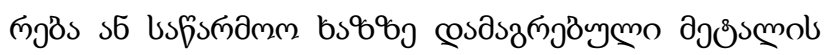

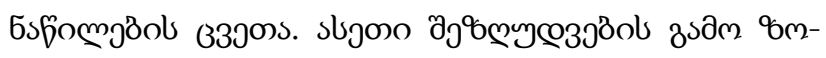

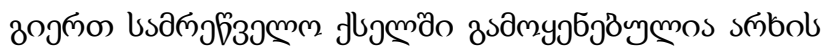

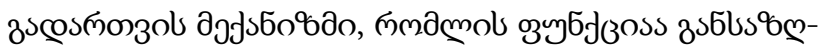

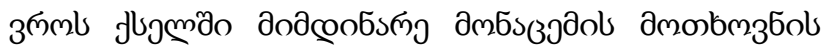

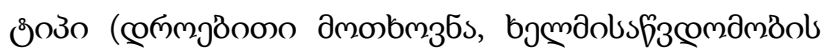




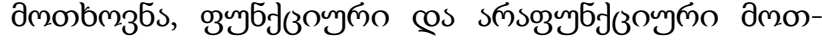

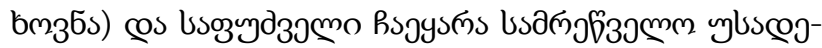

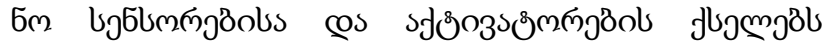
(IWSAN - Industrial Wireless Sensor and Actuator

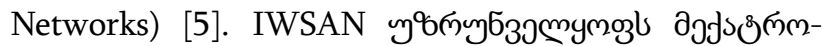

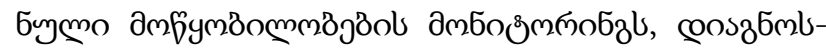

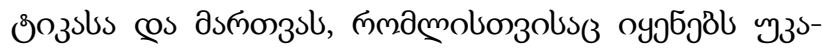

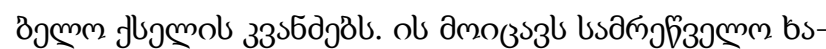

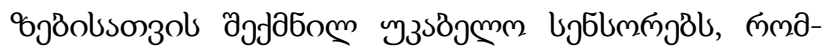

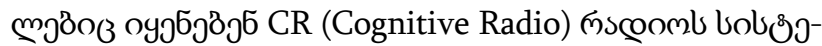

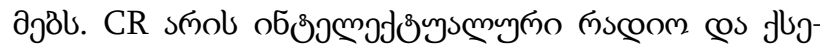

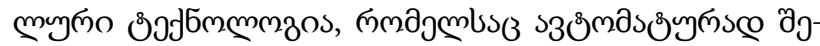

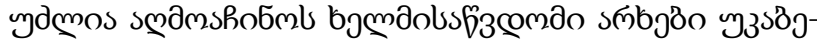

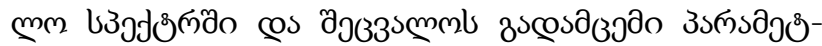

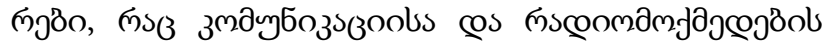

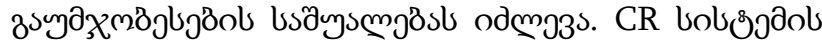

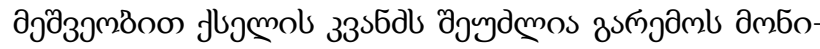

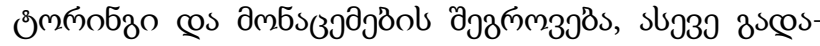

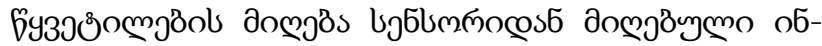

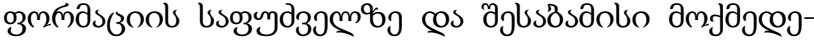

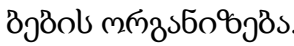

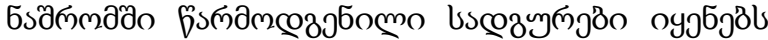

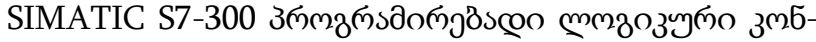

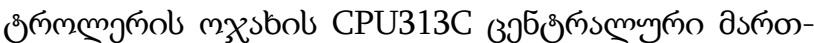

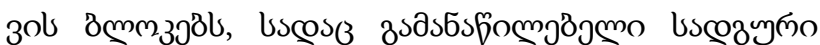

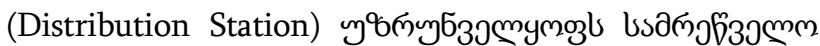

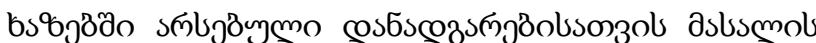

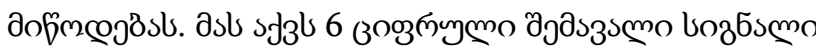

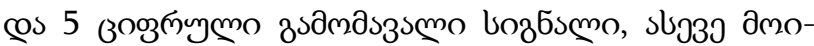

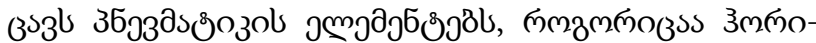

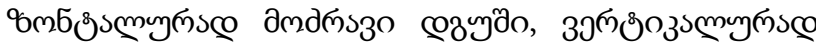

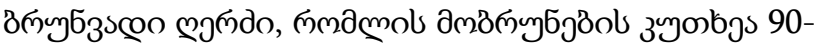

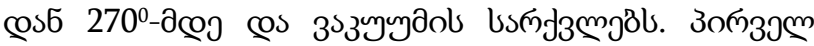

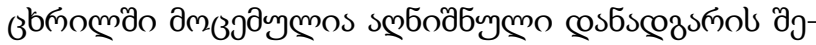

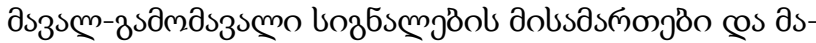

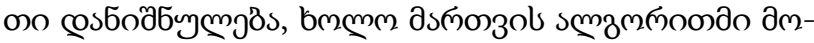

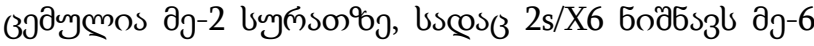

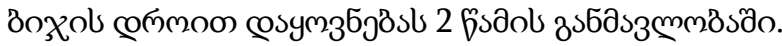

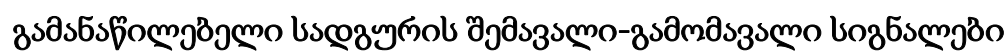

\begin{tabular}{|c|c|}
\hline \multicolumn{2}{|c|}{ 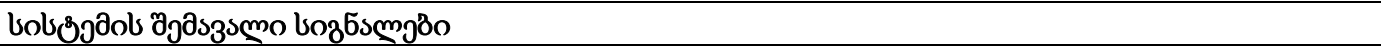 } \\
\hline бмадамо & cosбoz6ymmg8s \\
\hline I0 & 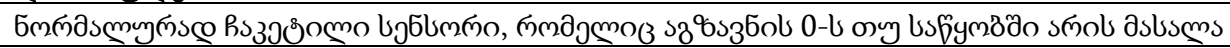 \\
\hline I1 & 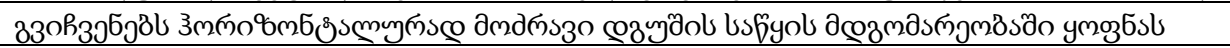 \\
\hline $\mathrm{I} 2$ & 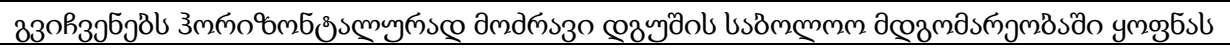 \\
\hline I3 & 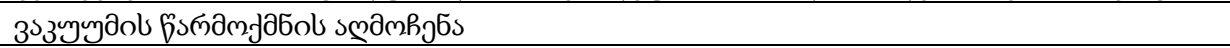 \\
\hline $\mathrm{I} 4$ & 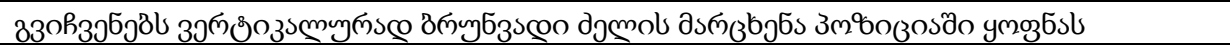 \\
\hline I5 & 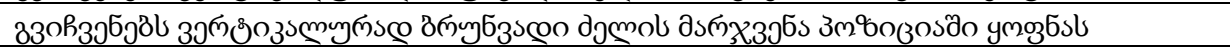 \\
\hline \multicolumn{2}{|c|}{ 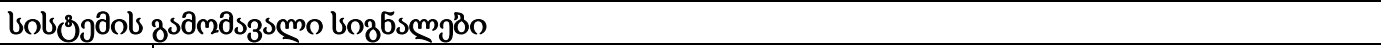 } \\
\hline бмадапо & cosбoz6ymmg8s \\
\hline Q0 & 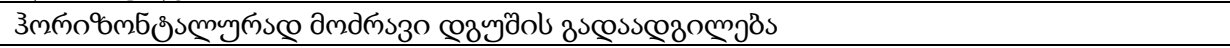 \\
\hline Q1 & 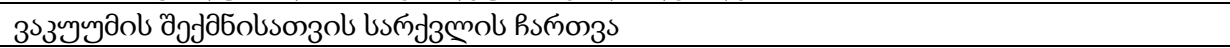 \\
\hline Q2 & 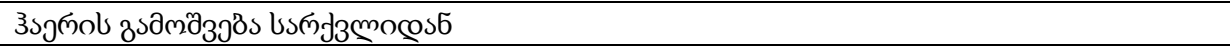 \\
\hline Q3 & 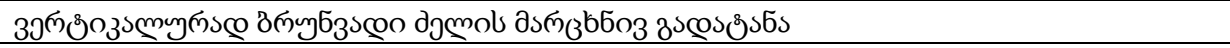 \\
\hline Q4 & 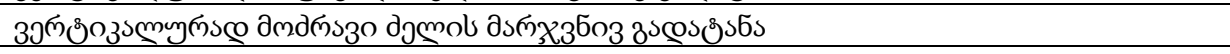 \\
\hline
\end{tabular}




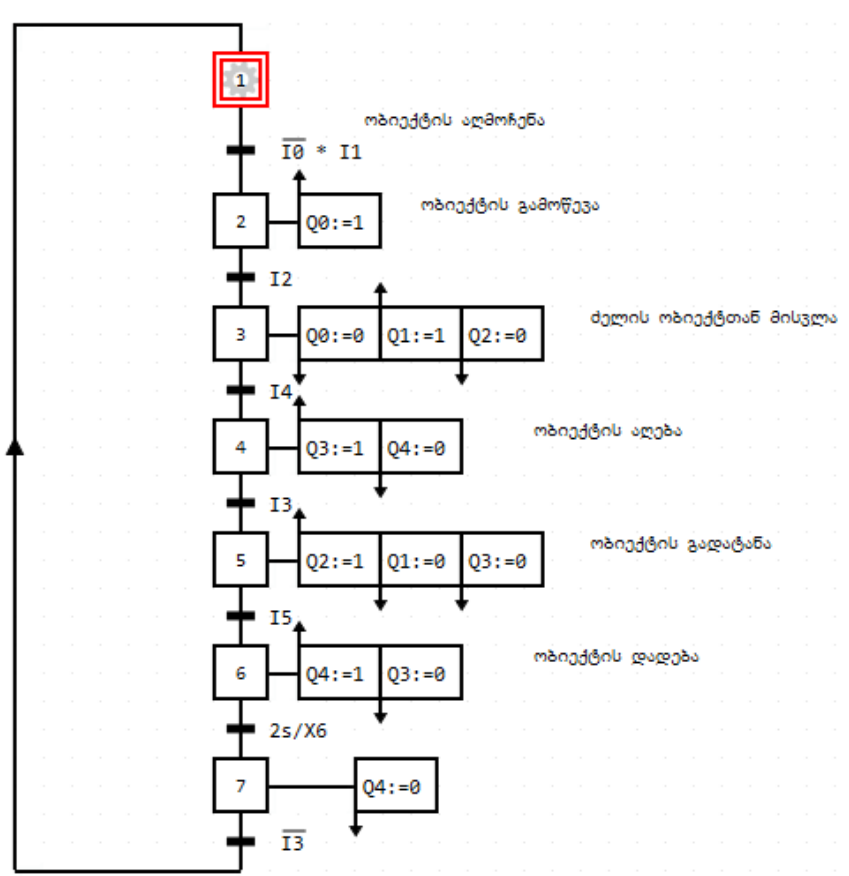

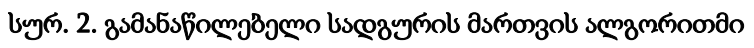

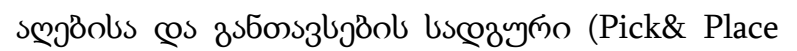

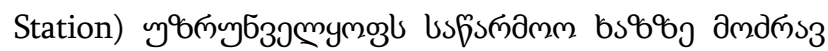

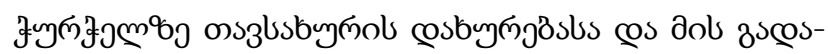

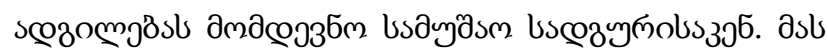

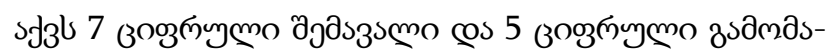

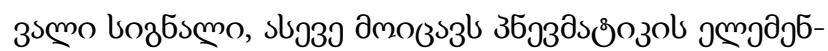

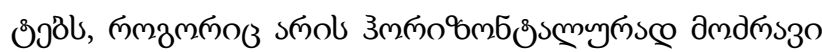

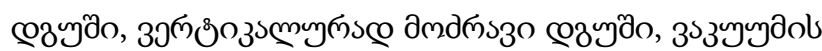

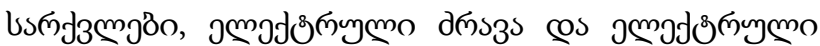

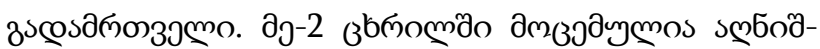

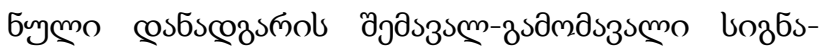

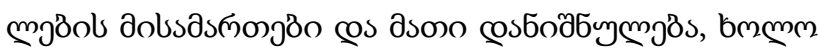

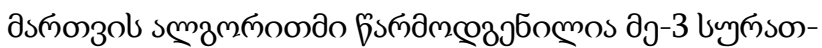

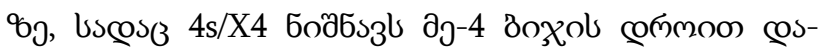

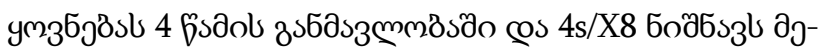

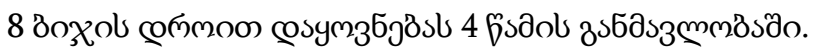

उвюомо 2

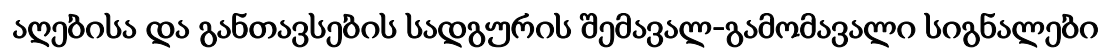

\begin{tabular}{|c|c|}
\hline \multicolumn{2}{|c|}{ 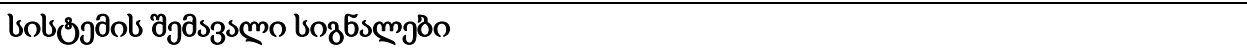 } \\
\hline бмдјщо & 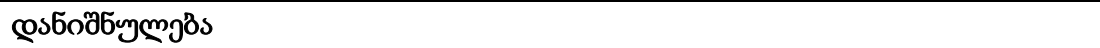 \\
\hline $\mathrm{I} 0$ & 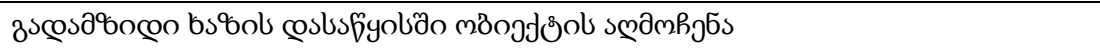 \\
\hline I1 & 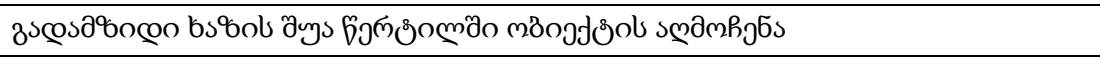 \\
\hline $\mathrm{I} 2$ & 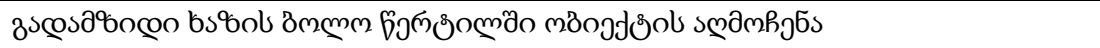 \\
\hline $\mathrm{I} 3$ & 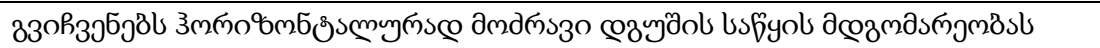 \\
\hline I4 & 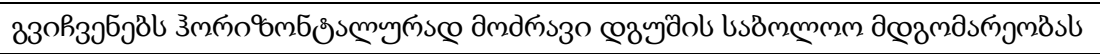 \\
\hline I5 & 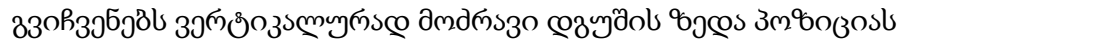 \\
\hline I6 & 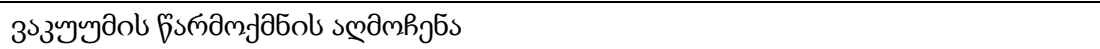 \\
\hline
\end{tabular}




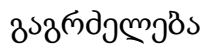

\begin{tabular}{|c|c|}
\hline \multicolumn{2}{|c|}{ 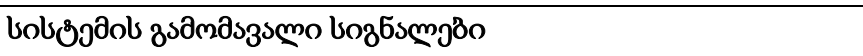 } \\
\hline бмдjӊo & 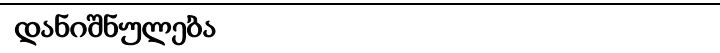 \\
\hline $\mathrm{Q} 0$ & 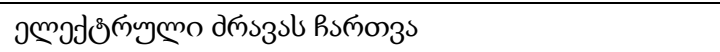 \\
\hline Q1 & 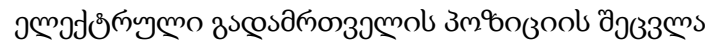 \\
\hline $\mathrm{Q} 2$ & 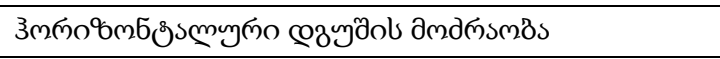 \\
\hline Q3 & 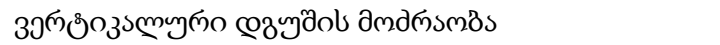 \\
\hline Q4 & 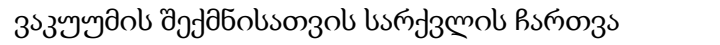 \\
\hline
\end{tabular}

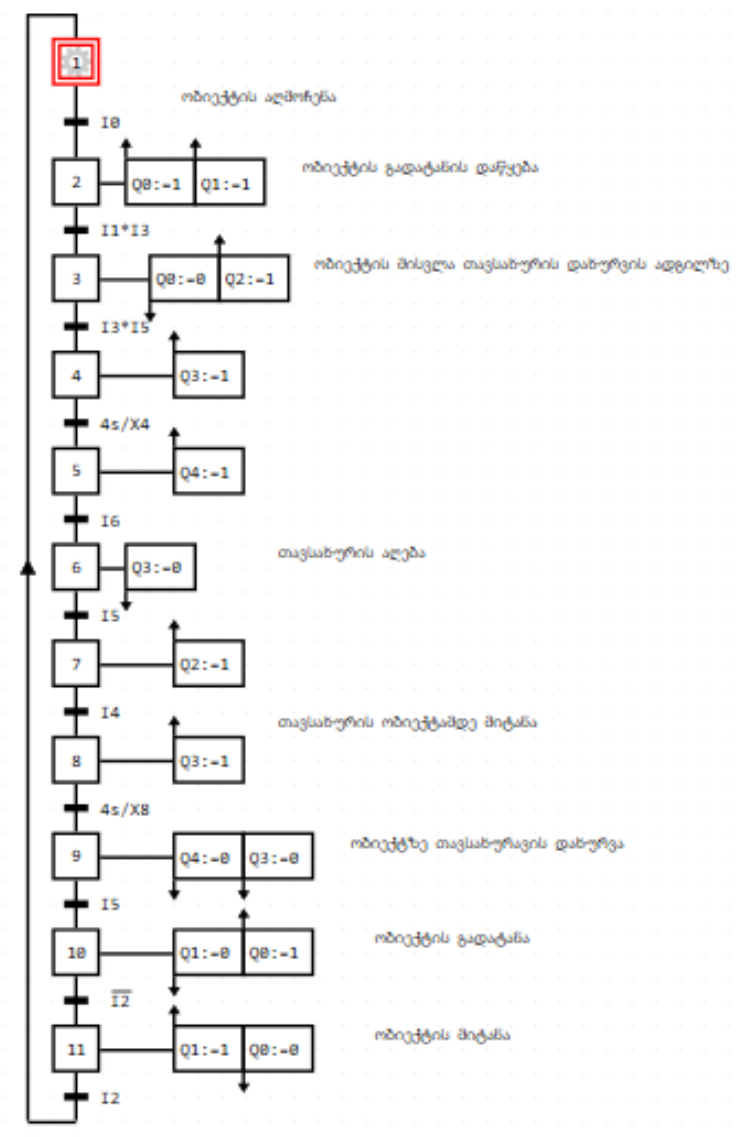

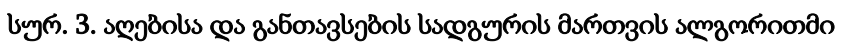

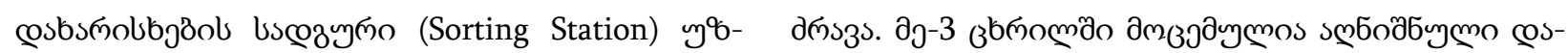

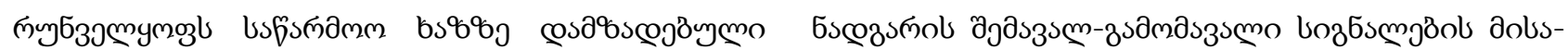

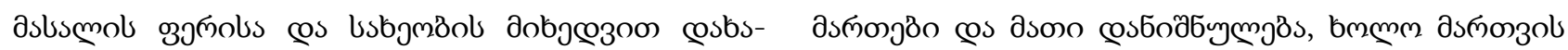

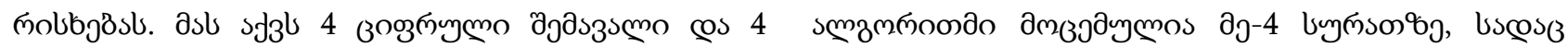

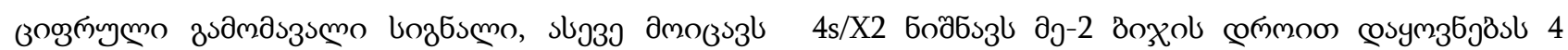

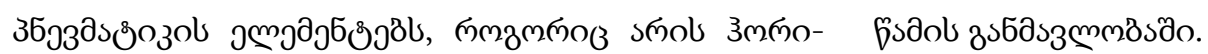

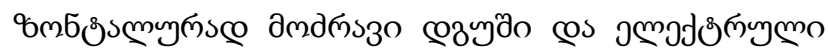




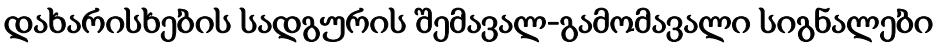

\begin{tabular}{|c|c|}
\hline \multicolumn{2}{|r|}{ 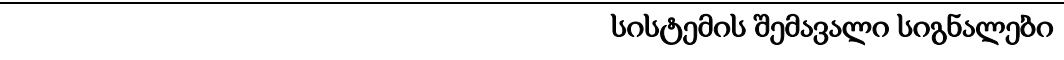 } \\
\hline бмдало & 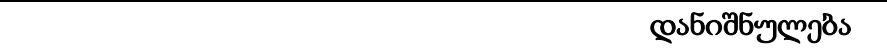 \\
\hline $\mathrm{I} 0$ & 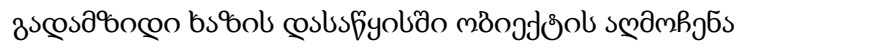 \\
\hline I1 & 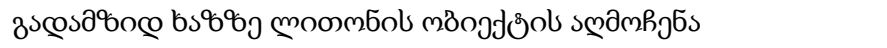 \\
\hline I2 & 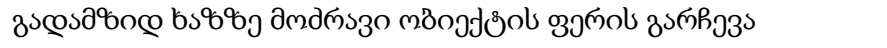 \\
\hline I3 & 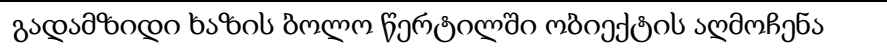 \\
\hline \multicolumn{2}{|c|}{ 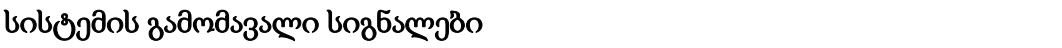 } \\
\hline бмдапо & 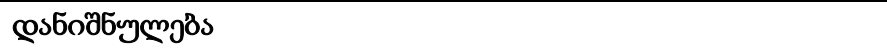 \\
\hline Q0 & 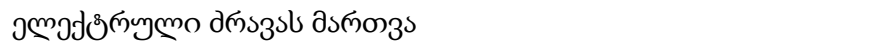 \\
\hline Q1 & 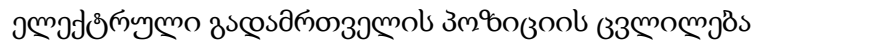 \\
\hline Q2 & 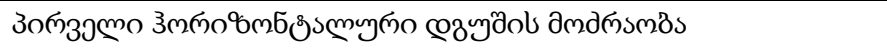 \\
\hline Q3 & 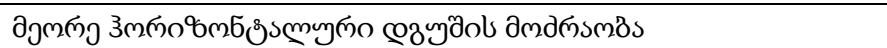 \\
\hline
\end{tabular}

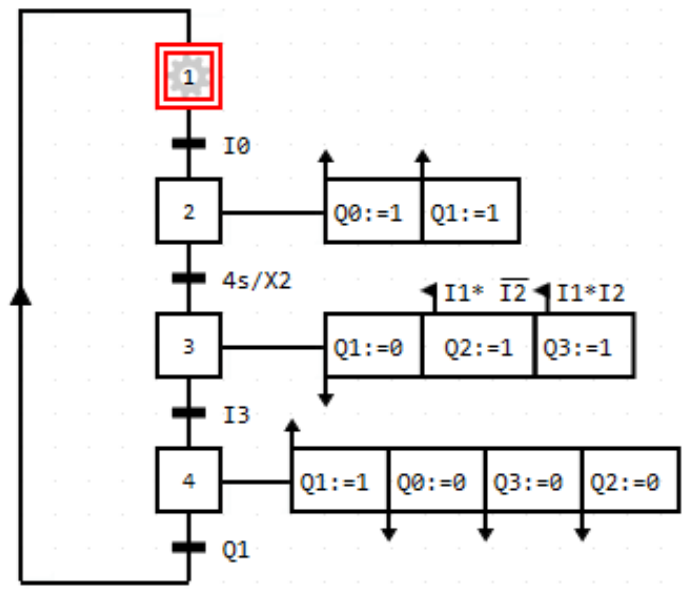

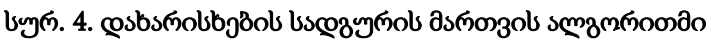

\section{cost336s}

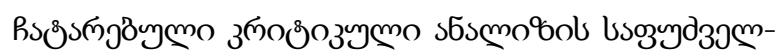

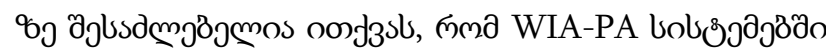

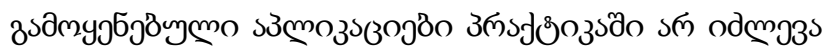

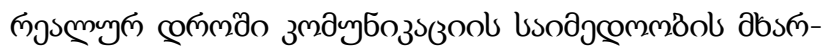

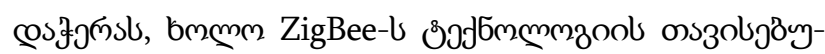

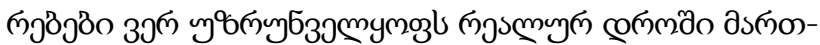

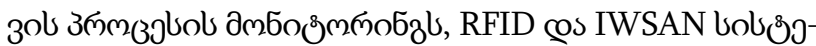

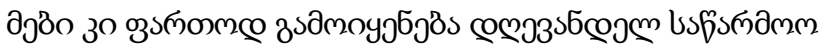

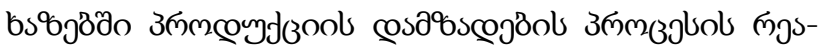

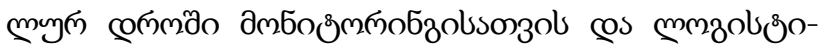

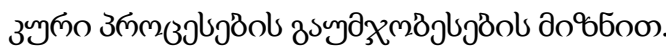




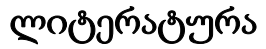

1. URL: https://www.controleng.com/articles/fundamentals-of-real-time-processing-in-automation-and-control/

2. Segura-Velandia D.M., Kaur N., Whittow W.G., Conway P.P., West, A.A.. Towards industrial internet of things: Crankshaft monitoring, traceability and tracking using RFID. 2018, 66-77 pp.

3. Sung W.T., Hsu Y.C. Designing an industrial real-time measurement and monitoring system based on embedded system and ZigBee. Expert Syst. Appl. 38(4). 2011, 4522-4529 pp.

4. das Neves Valadão Y., Künzel G., Müller I., Pereira C.E. Industrial wireless automation: Overview and evolution of WIA-PA. IFAC-PapersOnLine, 51(10). 2018, 175-180 pp.

5. Val I., Etxabe A., Torrego R., Rodriguez P.M., Cruces C., Diez V., Mendicute M., Arriola A. Design, analysis and implementation of a time-bounded spectrum handoff algorithm for real-time industrial wireless sensor and actuator networks. Journal of network and computer applications. 2018, 1-16 pp.

UDC 65.012.122:658.284

SCOPUS CODE 2209

DOI: https://doi.org/10.36073/1512-0996-2019-3-68-77

\section{Monitoring systems of industrial processes and control algorithms}

Salome Oniani

Ia Mosashvili
Department of Computer Engineering, Georgian Technical University, 77 M. Kostava str, 0160 Tbilisi, Georgia

E-mail: s.oniani@gtu.ge

Department of Computer Engineering, Georgian Technical University, 77 M. Kostava str, 0160 Tbilisi, Georgia

E-mail: i_mosashvili@gtu.ge

\section{Reviewers:}

K. Kotrikadze, Associate Professor, Faculty of Informatics and Control Systems, GTU

E-mail: ketino27@gmail.com

P. Jokhadze, Professor, Faculty of Informatics and Control Systems, GTU

E-mail: paata_j@yahoo.com

Abstract. The number of automated industries is increased significantly with the development of technology. At the beginning of this process, the aim of the automatization was increasing productivity and decreasing human resources. However, today the process is focused on the product quality and reliability of the manufacturing processing. The factories' owners started to set up networking systems and software for monitoring the production process in real time and maintenance of product quality. This paper presents a critical analysis of the systems used for the monitoring of the production process in real time and the control algorithms with three types of equipment developed by Festo Didactic.

Key words: Control algorithms of automated machines; monitoring systems; real time industrial systems. 
UDC 65.012.122:658.284

SCOPUS CODE 2209

DOI: https://doi.org/10.36073/1512-0996-2019-3-68-77

\title{
Системы мониторинга производственных процессов и алгоритмы управления
}

Саломе Ониани

Ия Мосашвили
Департамент компьютерной инжинерии, Грузинский технический университет, Грузия, 0160, Тбилиси, ул. М. Костава, 77

E-mail: s.oniani@gtu.ge

Департамент компьютерной инжинерии, Грузинский технический университет, Грузия, 0160, Тбилиси, ул. М. Костава, 77

E-mail: i_mosashvili@gtu.ge

\section{Рецензенты:}

К. Котрикадзе, ассоц. профессор факультета информатики и систем управления, ГТУ

E-mail: ketino27@gmail.com

P. Джохадзе, профессор факультета информатики и систем управления, ГТУ

E-mail: paata_j@yahoo.com

Аннотация. С развитием технологий число автоматизированных предприятий значительно увеличилось, и на первом этапе развития целью автоматизации было повышение производительности и сокращение человеческих ресурсов. Тем не менее, их основной задачей сегодня является повышение качества продукции и надежности ее производственного процесса. Вследствие этого владельцы заводов начали настраивать сетевые системы и программное обеспечение, благодаря которым появилась возможность контролировать процесс производства продукта и поддержание трения продукта. Вот почему в настоящем документе представлен критический анализ систем, используемых для мониторинга процесса производственных процессов в режиме реального времени, и модель управления тремя типами оборудования, созданного Festo Didactic.

Ключевые слова: автоматизированные модели управления оборудованием; промышленные системы реального времени; системы мониторинга.

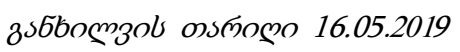

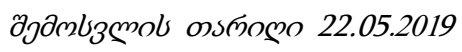

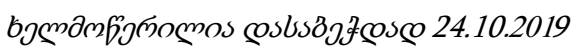


UDC $532.595: 534.833: 622.648$

SCOPUS CODE 2210

DOI: https://doi.org/10.36073/1512-0996-2019-3-78-85

Свободный, нерастворенный в жидкости воздух - эффективное средство для гашения гидравлических ударов в напорных гидротранспортных системах

\author{
Леон Махарадзе Департамент горной технологии, Грузинский технический университет, Грузия, \\ 0160, Тбилиси, ул. М. Костава 75 \\ E-mail: lmakharadze@gtu.ge
}

\title{
Рецензенты:
}

Г. Кирмелашвили, профессор факультета информатики и систем управления ГТУ

E-mail: gkirmelashvili@gtu.ge

А. Бежанишвили, профессор горно-геологического факультета ГТУ

E-mail: bezhanishvili@gmail.com

\begin{abstract}
Аннотащия. В настоящее время напорные гидротранспортные системы находят широкое распространение во многих отраслях промышленности из-за своих многих положительных сторон. Однако из-за специфики эксплуатации, в аналогичных системах часто возникают нестационарные процессы и гидравлические удары, которые отрицательно влияют на режим их работы, так как нередко являются причинами серьезных аварий. Исходя из этого, борьба против таких явлений является актуальной проблемой. Из многих средств и способов, разработанных нами, таким способом является свободный впуск нерастворенного в капельной жидкости воздуха в напорные гидротранспортные системы, который эффективно
\end{abstract}

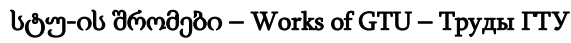
№3 (513), 2019 предотвращает возникновение гидравлических ударов, что подтверждено теоретическими и экспериментальными исследованиями, проведенными нами.

Ключевые слова: гидравлические удары; гидротранспортные системы; затухание процесса; концентрация воздуха; многофазный поток; нестационарные процессы; превышение давлений.

\section{Введение}

Со второй половины прошлого века напорные гидротранспортные системы, т.е. транспортирование различных твердых сыпучих материалов посредством потока капельной жидкости (в основном воды) по трубопроводам нашло широкое применение во www.shromebi.gtu.ge 
многих сферах промышленности и сельского хозяйства. Это обусловлено многими положительными свойствами по отношению к традиционным видам транспорта [1-7]. Кроме того, что этот вид транспорта очень чистый, в некоторых случаях, например, в горном деле, когда необходимо частичное или полное обогащение полезных ископаемых, с этим видом транспорта не может конкурировать ни один другой вид транспорта.

При напорном трубопроводном гидротранспорте по трубопроводам фактически всегда транспортируется трехфазная гидроаэросмесь (частицы твердых сыпучих материалов+несущая среда - капельная жидкость+ нерастворенный в жидкости воздух, которым обложены частицы твердого сыпучего материала). Особенно примечательным является то обстоятельство, что в процессе транспортирования, т.е. при перемещении по трубопроводу, в зависимости от изменения давления, могут измениться объемы составляющих элементов и соответственно их концентрации в данном сечении. Из-за этого обстоятельства по всей длине трубопроводной магистрали режим движения потока гидроаэросмеси не может быть полностью установившимся, что соответственно влияет как на характер протекания процесса, так и на величину изменения давления.

Из вышеизложенного видно, что процесс является весьма сложным, поэтому со второй половины прошлого века в Горном институте им. Г.А. Цулукидзе начались крупномасштабные фундаментальные исследования нестационарных процессов и гидравлических ударов в напорных трубопроводных гидротранспортных системах и с учетом полученных результатов разработка эффективных способов и средств для борьбы против этих нежелательных явлений [1-7], чтобы обеспечить надежную работу аналогичных систем в любом случае эксплуатации.

В настоящей работе рассматривается фрагмент из этих исследований, в котором отражены результаты исследований свободного, нерастворенного в жидкости воздуха, как эффективного средства для гашения гидравлических ударов в напорных трубопроводных гидротранспортных системах.

\section{Основная часть}

Как было сказано выше, нами были проведены фундаментальные исследования нестационарных процессов и гидравлических ударов в напорных гидротранспортных системах. Были проведены теоретические и экспериментальные исследования как на лабораторных, так и на промышленных установках. Для исследований в лабораторных условиях специально были смонтированы полупромышленные установки. В теоретической части исследования были основаны на теории Н.Е. Жуковского [8]. По этой теории основным параметром существенно влияющим как на характер протекания в трубопроводах, так и на величину превышения давления над давлением при установившемся режиме, является скорость распространения волны возмущения при неустановившихся процессах. Исходя из этого, нами на лабораторных экспериментальных установках были проведены исследования по ее определению при транспортировании двухфазных (частицы твердых сыпучих материалов+несущая среды - капельная жидкость) и трехфазных (частицы твердых сыпучих материалов+несущая среда - капельная жидкость+ сво- 
бодный нерастворенный в жидкости воздух) потоков. На величину скорости распространения волны гидравлического удара в трубопроводе при транспортировании трехфазных гидроаэросмесей существенно влияет наличие свободного, нерастворенного в капельной жидкости воздуха, объемный модуль упругости которого значительно меньше объемного модуля упругой несущей капельной жидкости, как правило, воды, тем более твердых сыпучих материалов. C целью определения степени влияния объема свободного, нерастворенного в капельной жидкости воздуха на скорость распространения волны гидравлического удара, на лабораторных установках в трубопроводах внутренними диаметрами 51,5; 68, 81, 104 и 146 мм были проведены специальные исследования, результаты которых сведены в таблице 1. По этим данным построены экспериментальные кривые, которые приведены в монографии [7].

Опыты были проведены при транспортировании чистой воды, двухфазных потоков (вода+нераство- ренный в ней воздух, и вода+частицы твердых сыпучих материалов и трехфазного потока (вода+нерастворенный в нем воздух+частицы твердых сыпучих материалов).

На основе анализа экспериментальных исследований нами получена эмпирическая зависимость для определения скорости распространения волны гидравлического удара в трубопроводах при транспортировании двухфазного потока (вода - капельная жидкость+свободный, нерастворенный в капельной жидкости воздух) в зависимости от концентрации воздуха в потоке, который имеет вид:

$$
a_{\text {эмn. }}=\frac{0,049 \cdot a_{\text {mеор. }}}{K_{\text {воз. }}^{1,86}+0,065}+a_{\text {воз. }},
$$

где $a_{\text {воз. }}$ - скорость распространения волны гидравлического удара в воздухе, м/с; $a_{\text {mеор. }}$ - теоретическое значение скорости волны гидравлического удара в трубопроводе,

Результаты экспериментальных исследований эффективности влияния свободного, нерастворенного в капельной жидкости воздуха, на степень гашения гидравлического удара в трубопроводах

\begin{tabular}{|c|c|c|c|c|c|c|c|}
\hline 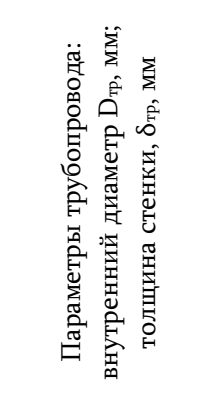 & 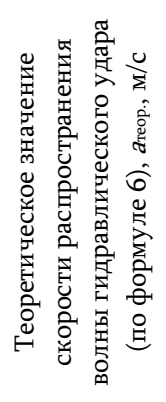 & 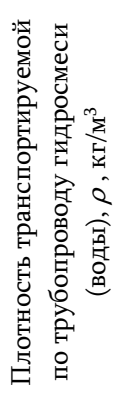 & 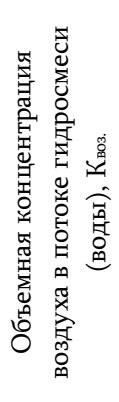 & 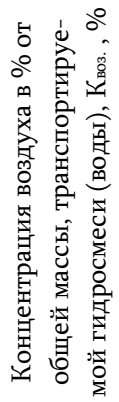 & 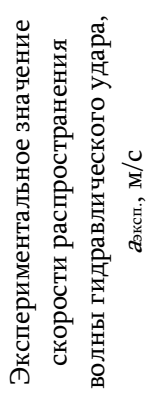 & 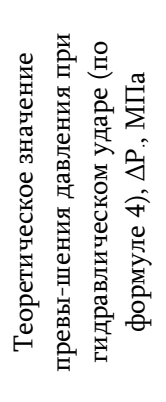 & 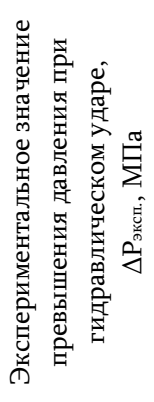 \\
\hline 1 & 2 & 3 & 4 & 5 & 6 & 7 & 8 \\
\hline $\mathrm{D}_{\mathrm{\tau p}}=51,5 ; \delta_{\mathrm{Tp}}=6$ & 1368 & 1000 & 0,005 & 0,5 & 575 & 3,42 & 1,40 \\
\hline$-\ll-$ & 1368 & 1150 & 0,001 & 0,1 & 1150 & 3,93 & 3,15 \\
\hline $\begin{array}{ll}-\ll- \\
\end{array}$ & 1368 & 1150 & 0,003 & 0,3 & 1020 & 3,93 & 2,86 \\
\hline
\end{tabular}


продолжение

\begin{tabular}{|c|c|c|c|c|c|c|c|}
\hline$-\ll-$ & 1368 & 1150 & 0,005 & 0,5 & 630 & 3,93 & 1,82 \\
\hline$-\ll-$ & 1368 & 1150 & 0,008 & 0,8 & 480 & 3,93 & 1,35 \\
\hline$-\ll-$ & 1368 & 1150 & 0,010 & 1,0 & 440 & 3,93 & 1,21 \\
\hline $\mathrm{D}_{\mathrm{Tp}}=68 ; \delta_{\mathrm{Tp}}=4$ & 1368 & 1000 & 0,005 & 0,5 & 540 & 3,29 & 1,38 \\
\hline$-\ll-$ & 1368 & 1150 & 0,001 & 0,1 & 1140 & 3,79 & 3,25 \\
\hline$-\ll-$ & 1368 & 1150 & 0,003 & 0,3 & 780 & 3,79 & 2,25 \\
\hline$-\ll-$ & 1368 & 1150 & 0,005 & 0,5 & 620 & 3,79 & 1,70 \\
\hline 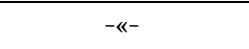 & 1368 & 1150 & 0,008 & 0,8 & 510 & 3,79 & 1,52 \\
\hline$-\ll-$ & 1368 & 1150 & 0,010 & 1,0 & 460 & 3,79 & 1,30 \\
\hline $\mathrm{D}_{\text {тр }}=81 ; \delta_{\text {тр }}=4,25$ & 1307 & 1000 & 0,005 & 0,5 & 520 & 2,61 & 1,05 \\
\hline $\begin{array}{l}-\ll- \\
\end{array}$ & 1307 & 1150 & 0,0012 & 0,12 & 1050 & 3,00 & 2,40 \\
\hline$-\ll-$ & 1307 & 1150 & 0,0017 & 0,17 & 950 & 3,00 & 2,20 \\
\hline$-\ll-$ & 1307 & 1150 & 0,0038 & 0,38 & 680 & 3,00 & 1,50 \\
\hline$-\ll-$ & 1307 & 1150 & 0,0053 & 0,53 & 550 & 3,00 & 1,30 \\
\hline$-\ll-$ & 1307 & 1150 & 0,0099 & 0,99 & 410 & 3,00 & 0,92 \\
\hline $\mathrm{D}_{\text {тр }}=104 ; \delta_{\text {тр }}=5$ & 1298 & 1000 & 0,005 & 0,5 & 500 & 3,24 & 1,31 \\
\hline$-\ll-$ & 1298 & 1150 & 0,001 & 0,1 & 1080 & 3,73 & 3,10 \\
\hline 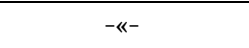 & 1298 & 1150 & 0,003 & 0,3 & 750 & 3,73 & 2,25 \\
\hline$-\ll-$ & 1298 & 1150 & 0,005 & 0,5 & 570 & 3,73 & 1,52 \\
\hline$-\ll-$ & 1298 & 1150 & 0,008 & 0,8 & 460 & 3,73 & 1,30 \\
\hline $\begin{array}{lll}-\ll- & -1 \\
\end{array}$ & 1298 & 1150 & 0,010 & 1,0 & 430 & 3,73 & 1,18 \\
\hline $\mathrm{D}_{\mathrm{Tp}}=146 ; \delta_{\mathrm{Tp}}=11,5$ & 1339 & 1000 & 0,005 & 0,5 & 420 & 2,68 & 0,82 \\
\hline$-\ll-$ & 1339 & 1150 & 0,0014 & 0,14 & 940 & 3,08 & 2,20 \\
\hline $\begin{array}{lll}- & - \\
\end{array}$ & 1339 & 1150 & 0,0019 & 0,19 & 840 & 3,08 & 1,92 \\
\hline$-\ll-$ & 1339 & 1150 & 0,0038 & 0,38 & 600 & 3,08 & 1,40 \\
\hline$-\ll-$ & 1339 & 1150 & 0,0086 & 0,86 & 390 & 3,08 & 0,90 \\
\hline$-\ll-$ & 1339 & 1150 & 0,0120 & 1,20 & 360 & 3,08 & 0,81 \\
\hline
\end{tabular}

определенное согласно теории Н.Е. Жуковского [8], м/с; $K_{\text {воз. }}$ - концентрация воздуха в потоке капельной жидкости - воды.

На основе анализа этих же экспериментальных исследований нами получена эмпирическая зависимость для определения скорости распространения волны гидравлического удара в трубопроводах при транспортировании трехфазного потока (вода - капельная жидкость+твердые частицы твердых сыпучих материалов+свободный, нерастворенный в капельной жидкости воздух), которая имеет вид:

$$
a_{\text {эмп. }}=\frac{0,49 \cdot a_{\text {mеор. }}}{K_{\text {воз. }}^{1,86} \cdot K_{c \text { c. }}^{1,05}+0,065}+a_{\text {воз. }}
$$

где $K_{c м}$ - концентрация твердого сыпучего материала в потоке гидроаэросмеси.

На основании наших исследований установлено, что при транспортировании твердых частиц сыпучих материалов скорость теоретического значения этого же параметра для данного трубопровода всегда меньше теоретического значения этого же параметра, определенного по формуле Н.Е. Жуковского. Эта разница (в сторону уменьшения) увеличивается с уве- 
личением концентрации гидросмеси, то есть с увеличением насыщения потока воды частицами твердого сыпучего материала. Это можно объяснить тем, что с твердыми частицами сыпучих материалов в трубопровод все же попадает определенный объем свободного, нерастворенного в воде воздуха. При увеличении же концентрации воздуха в потоке водовоздушной смеси, увеличение насыщенности последнего частицами твердого материала практически не влияет на скорость распространения волны гидравлического удара.

На основании анализа экспериментальных исследований нами получена эмпирическая зависимость для определения скорости распространения волны гидравлического удара для данного трубопровода, в зависимости от объемной концентрации гидросмеси, которая имеет вид:

$$
a_{\text {эмп. }}=a_{\text {mеор. }} \exp \left(-1,2 \cdot 8,5 D_{m p} S_{c M}\right),
$$

где $D_{m p}$ - внутренний диаметр трубопровода, мм; $S_{c M}$ - объемная концентрация гидросмеси.

Справедливость результатов, полученных нами на лабораторных установках нами проверена на крупных промышленных установках. Эти исследования позволили установить оптимальный объем свободного, нерастворенного в капельной жидкости (в воде) воздуха, впуск которого в трубопроводы обеспечивает снижение скорости распространения волны гидравлического удара для данного трубопровода до такой степени, что повышение давлений при гидравлических ударах не превышало допустимых значений. Для этого достаточно в трубопроводы впустить воздух под атмосферным давлением в объеме 0,25 -
1,5 \% от общего объема транспортируемой по трубопроводу гидросмеси [10], подтверждением этого являются осциллограммы, приведенные на рис. 1.

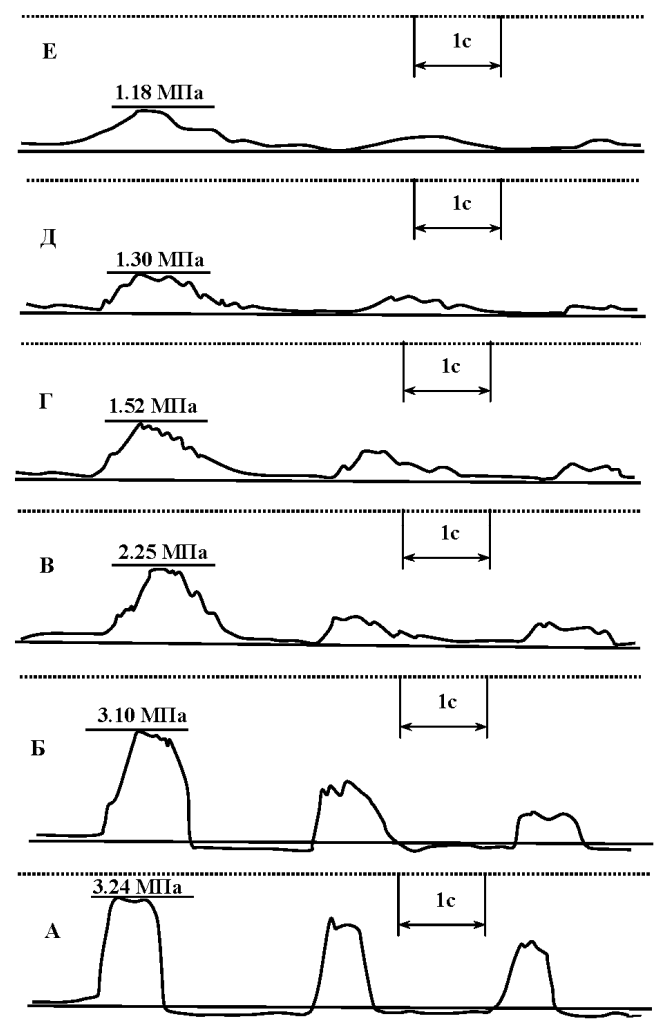

Рис. 1. Осциллограммы гидравлических ударов в трубопроводе $\mathrm{D}_{\text {тр}}=104$ мм, при транспортировании гидросмеси плотностью $\rho_{\mathrm{cm}}$ $=1100$ кг $/ \mathrm{cm}^{3}$ со скоростью vo $=2,5 \mathrm{~m} / \mathrm{c}$, скорость распространения волны гидравлического удара (экспериментальное значение) аэкп. $=1180$ м/с: А - прямой гидравлический удар; Б - при

концентрации воздуха в потоке Квоз. $=0,1 \%$; В - при Квоз. $=0,3 \%$; $Г$ при Квоз. $=0,5 \%$; Д - при Квоз. $=0,8 \%$; Е - при Квоз. $=1,0 \%$

Основным параметром при определении изменения давления во время нестационарных процессов и гидравлических ударов, согласно теории Н.Е. Жуковского [8], является скорость распространения волны возмущения в напорных трубопроводах, так как превышение давления $\Delta P$ при таких процессах определяется по зависимости 


$$
\Delta P=a v_{0} \rho,
$$

$a$ - скорость распространения волны гидравлического удара в напорном трубопроводе, м/с; vo скорость движения потока жидкой массы в трубопроводе при установившемся режиме, м/с; $\rho$ - плотность, транспортируемой по трубопроводу жидкой массы, кг/ $\mathbf{M}^{3}$.

Полное давление при указанных выше процессах в напорном трубопроводе определяется по формуле

$$
P=P_{\text {раб. }}+\Delta P
$$

где $P_{\text {раб. }}$ рабочее давление в напорном трубопроводе при указанных процессах, Па;

$$
a_{\text {meop. }}=\frac{1425}{\sqrt{1+\frac{\varepsilon_{B}}{E} \cdot \frac{D_{m p}}{\delta_{m p}}}},
$$

$\varepsilon_{\text {в }}$ объемный модуль упругости воды, $\varepsilon_{6}=2,1 \cdot 10^{3} M \Pi a ; E-$ модуль упругости материала (стали) из которого изготовлен трубопровод, $E=2,1 \cdot 10^{5} M \Pi a ; \delta_{m p}$ - толщина стенки трубопровода, мм.

\section{Заключение}

Как отмечалось, напорный трубопроводный гидротранспорт из-за многих положительных свойств находит большое распространение во многих сферах промышленности и сельского хозяйства. Недостатком аналогичных систем является то обстоятельство, что из-за специфики эксплуатации, при эксплуатации часто возникают нестационарные процессы и гидравлические удары, которые отрицательно влияют на их надежность и технико-экономические показатели.

В Горном институте им. Г.А. Цулукидзе выполнены крупномасштабные теоретические и экспериментальные исследования по защите напорных гидротранспортных систем от резких повышений давления возникших при нестационарных процессах и гидравлических ударах. Разработаны многие средства и способы в этой области, новизна и полезность которых защищены авторскими свидетельствами и патентами.

Из этих способов наиболее эффективными являются свободный, нерастворенный в капельной жидкости воздух. Экспериментальными исследованиями установлено, что достаточно в напорные трубопроводы гидротранспортных систем впустить воздух под атмосферным давлением в объеме $0,25-1,5 \%$ от общего объема транспортируемой по трубопроводу гидросмеси, чтобы полностью предотвратить возможность возникновения резких колебаний давления.

\section{Литература}

1. Makharadze L.I. Effective remedies of protection of pressure pipelines against water hammers. Domestic and foreign experience. TSINIS. Moscow. 1979, 67 p. (in Russian).

2. Makharadze L.I. Protection of hydrotransport systems against hydraulic impact. Tbilisi: "Metsniereba". 1996, 150 p. (in Russian).

3. Makharadze L.I., Kirmelashvili G.I. Hydraulic impact in pipelines at transportation of multiphase hydromixes. Tbilisi: "Metsniereba”. 1997, 232 p. (in Russian). 
4. Dmitriev G., Makharadze L., Gochitashvili T. Pressure hydrotransport system. Manual. Moscow: "Nedra”. 1991, 304 p. (in Russian).

5. Makharadze L.I. The guide for protection of the pressure hydrotransport systems against water hammers of BCH 01-81. Tbilisi: "Metsniereba". 1981, 151 p. (in Russian).

6. Makharadze L.I. Means for prevention of fluctuation and deformations of pipelines. Construction of pipelines. №10. Moscow. 1974, 31-32 pp. (in Russian).

7. Makharadze L.I., Kirmelashvili G.I. Nonstationary processes in foreign hydrotransport systems and protection from water hammers. Tbilisi: "Metsniereba". 1986, 152 p. (In Russian).

8. Zhukovsky N.E. About water hammer in water pipes. Moscow-Leningrad: "Gostekhteoretizdat". 1949, 104 p. (in Russian).

9. Makharadze L.I., Gochitashvili T.Sh., Sulaberidze D.G. Rational method of calculation of hydrotransport systems pipelines. Moscow: "Construction of pipelines”. № 8. 1980, 29-30 pp. (in Russian).

10. Makharadze L.I. and others. The method to prevent water hammers in slurry pipelines and conduits. Patent 501245. USSR. Bulletin №4. 1976. (In Russian).

UDC $532.595: 534.833: 622.648$

SCOPUS CODE 2210

DOI: HTTPS://DOI.ORG/10.36073/1512-0996-2019-3-78-85

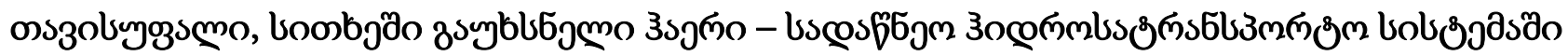

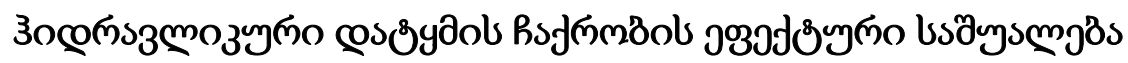

momb asbsmsdo

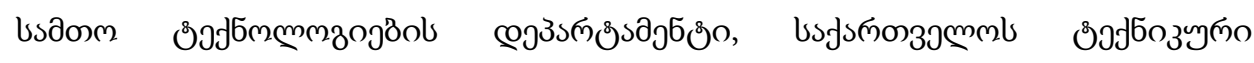

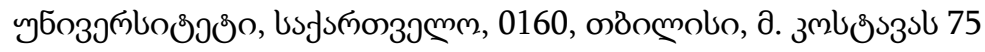 \\ E-mail: Imakharadze@gtu.ge
}

\section{๓๐สว}

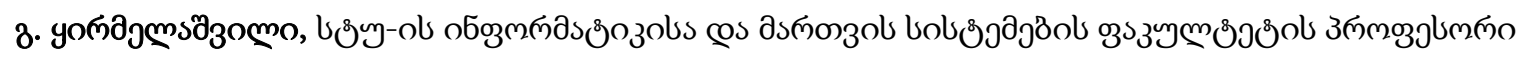

E-mail: gkirmelashvili@gtu.ge

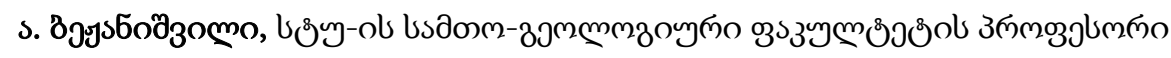

E-mail: bezhanishvili@gmail.com

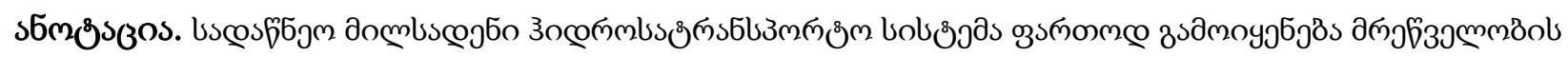

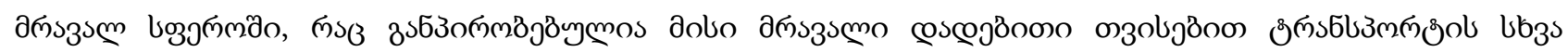

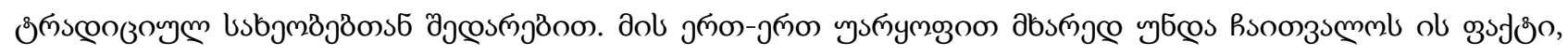

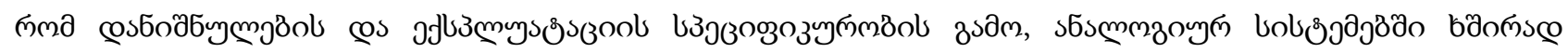

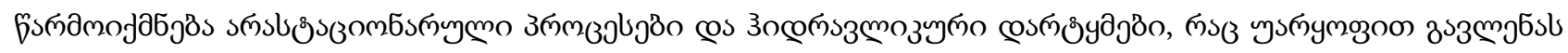

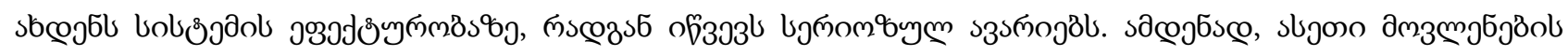

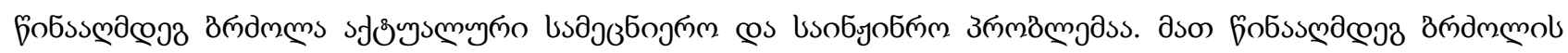




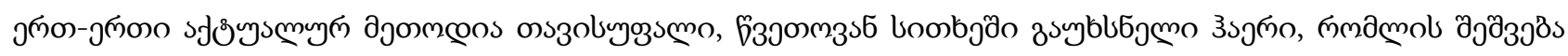

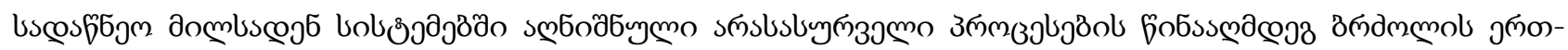

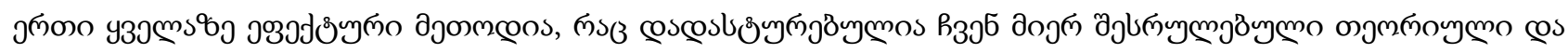

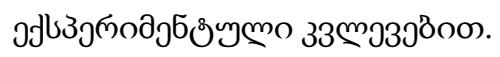

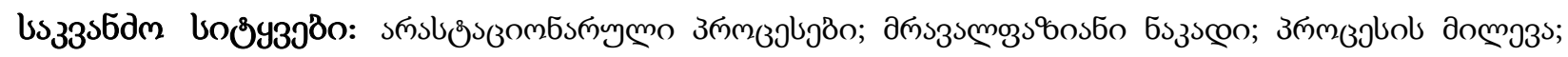

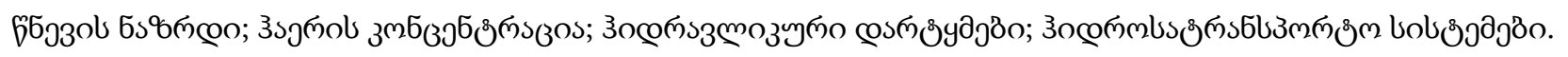

UDC $532.595: 534.833: 622.648$

SCOPUS CODE 2210

DOI: HTTPS://DOI.ORG/10.36073/1512-0996-2019-3-78-85

\section{Free combined air - as an effective remedy for water hammers attenuation in the pressure head hydrotransport systems}

Leon Makharadze Department of Mining Technology, Georgian Technical University, 75 M. Kostava str. 0160, Tbilisi, Georgia

E-mail: lmakharadze@gtu.ge

\section{Reviewers:}

G. Kirmelashvili, Professor, Faculty of Informatics and Control Systems, GTU

E-mail: gkirmelashvili@gtu.ge

A. Bezhanishvili, Professor, Faculty of Mining and Geological, GTU

E-mail: bezhanishvili@gmail.com

Abstract. For now the pressure head hydrotransport systems are used in many industries because of a number of their advanced features. However, because of specific operations and exploitation, there are occurred nonstationary processes and water hammers in similar systems, which have a negative effect on the system performance and often represent the reasons of serious accidents. Obviously it's an actual problem nowadays. To solve this problem we have developed the method of free combined air induction in the pressure head hydrotransport systems which effectively prevents formation of water hammers. It is proved by conducted theoretical and experimental researches and obtained results considered in this article.

Key words: Air-concentration; excess pressure; hydrotransport systems; multiphase flow; nonstationary processes; process attenuation; water hammers.

Дата рассмотрения 08.05.2019

Дата поступления 13.05.2019

Подписано к печати 24.10.2019 
UDC 66.074.32

SCOPUS CODE 2310

DOI: https://doi.org/10.36073/1512-0996-2019-3-86-97

\section{sళmతి 33लO3s}

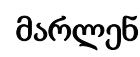

әђэщмодзомо

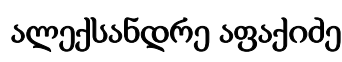

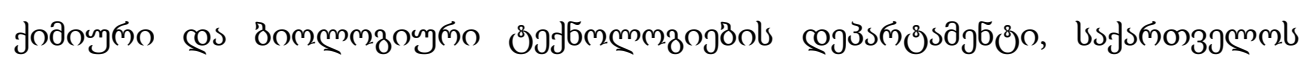

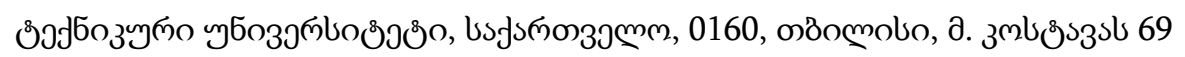
E-mail: m.mchedlishvili@gtu.ge

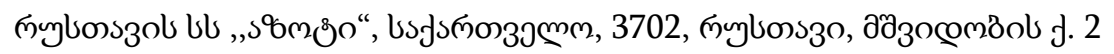
E-mail: alika.aphak@gmail.com

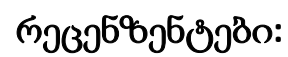

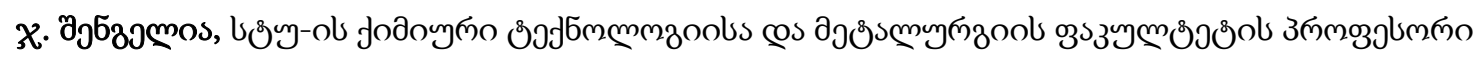
E-mail: jemal.shengelia@gtu.ge

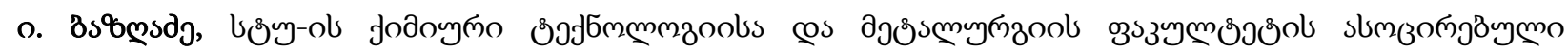
зпмозымпо

E-mail: i.bazgadze@gtu.ge

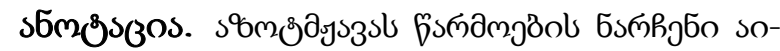

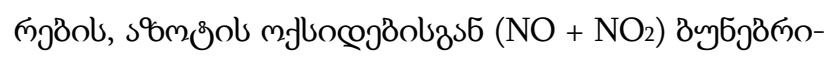

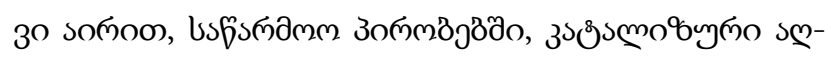

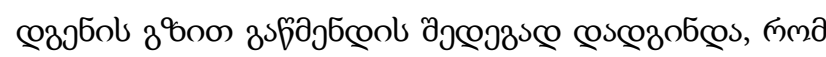

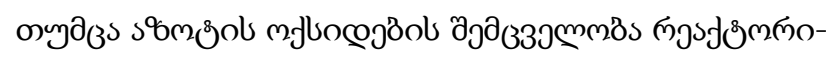

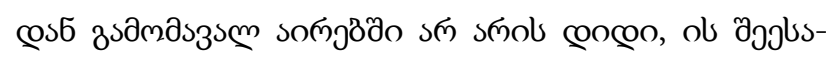

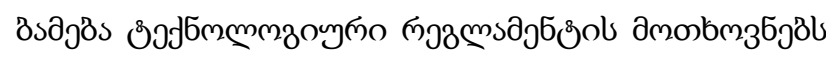

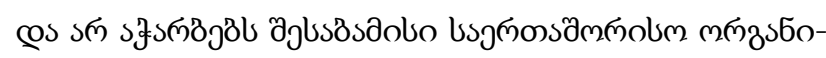

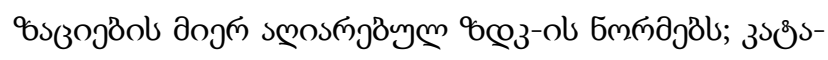

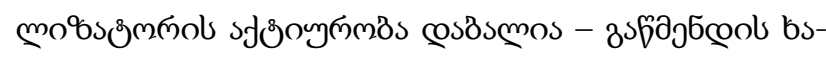

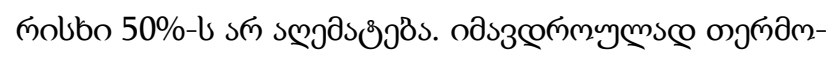

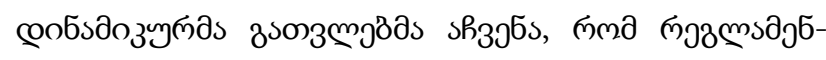

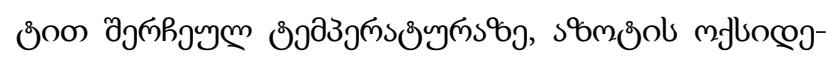

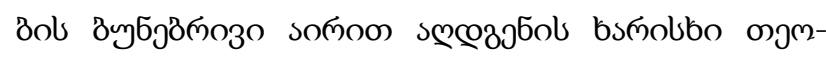
๓огумьs 99,97\%-оs.

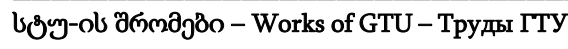
№3 (513), 2019

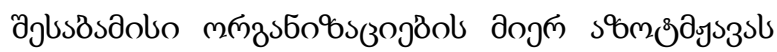

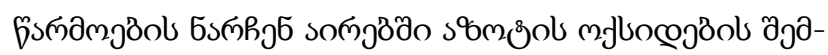

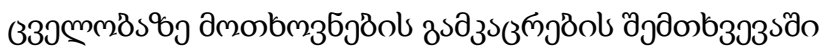

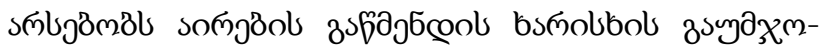

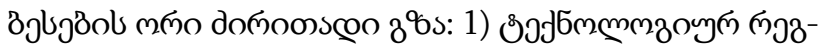

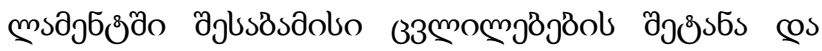

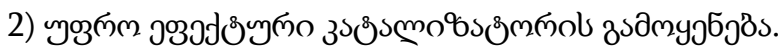

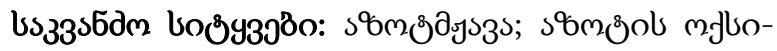

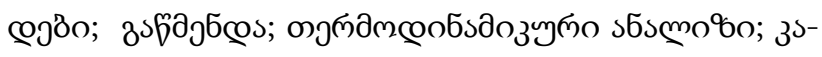
8s mos

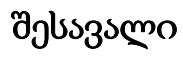

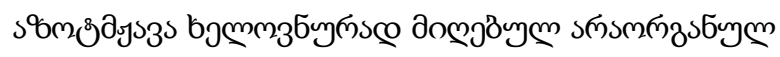

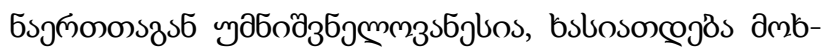




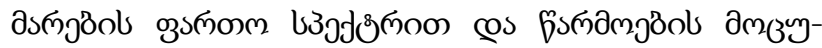

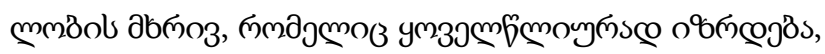

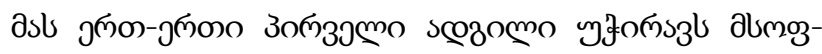

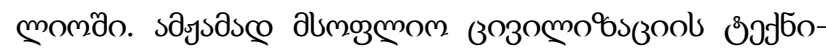

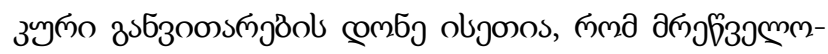

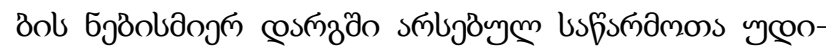

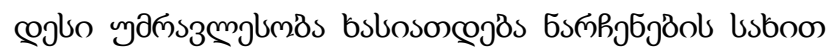

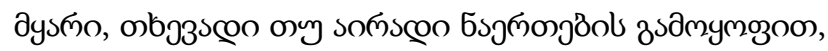

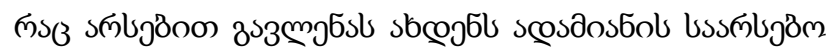

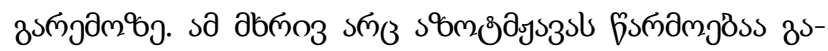

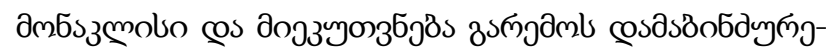

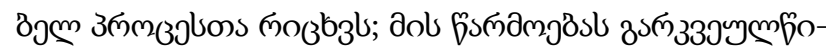

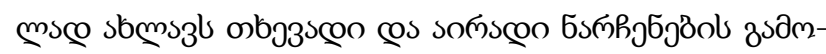

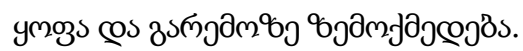

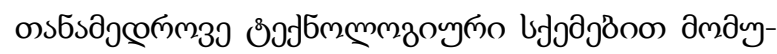

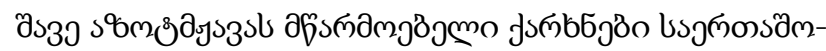

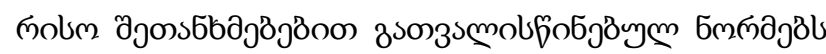
sзазумозому

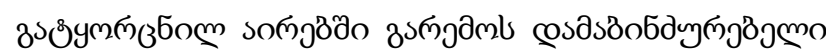

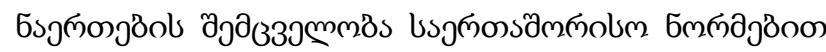

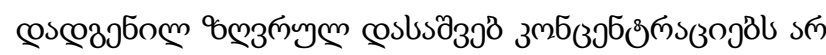

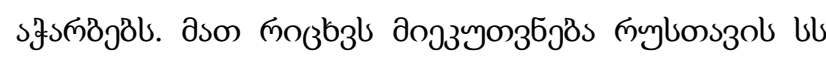

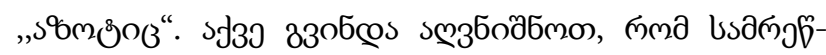

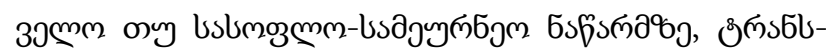

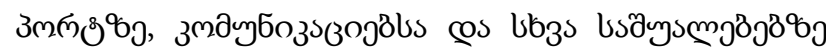

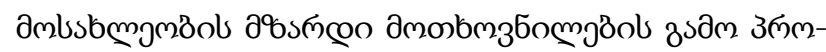

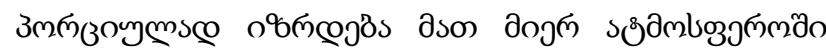

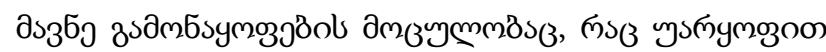

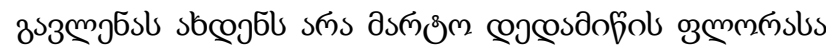

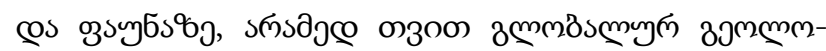

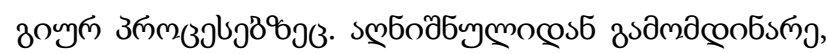

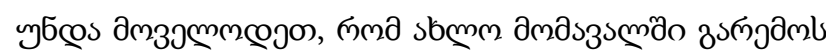

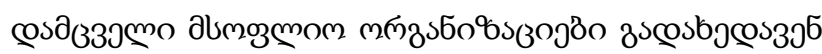

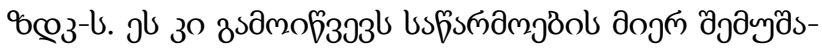

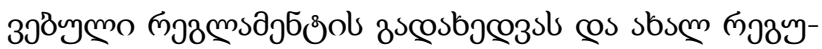

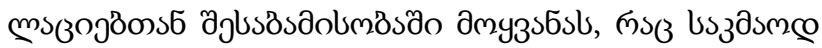

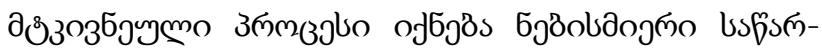

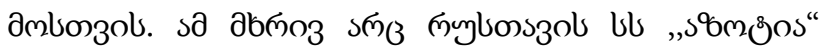

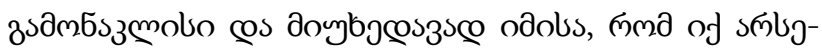

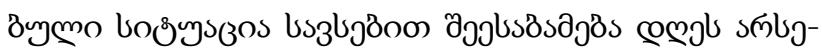

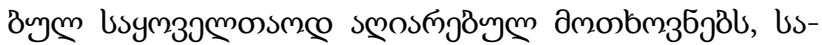

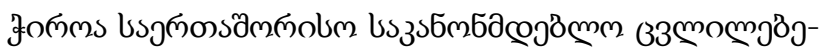

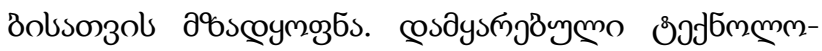

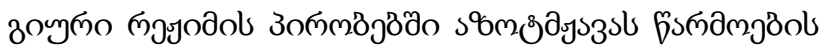

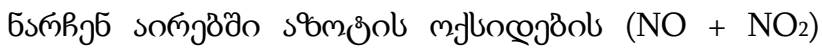

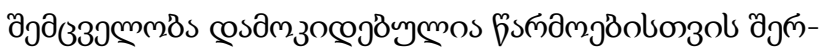

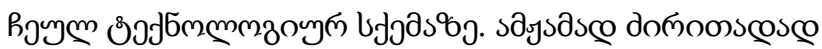

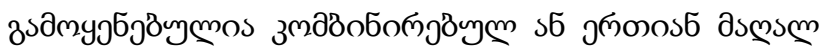
бБэзs\%

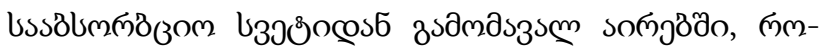

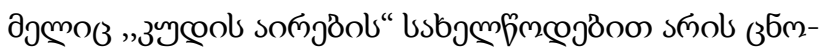

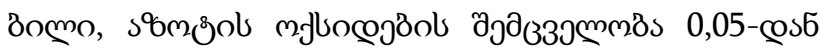

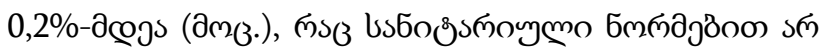

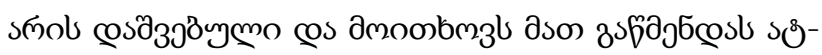

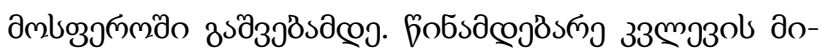

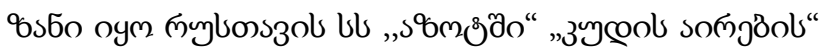

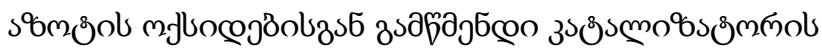

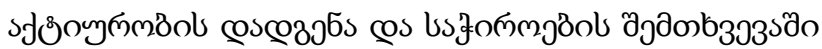

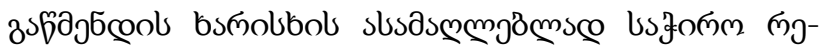

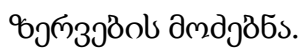

\section{допоonscen 6sfomo}

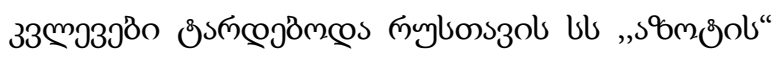

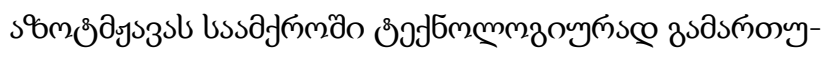

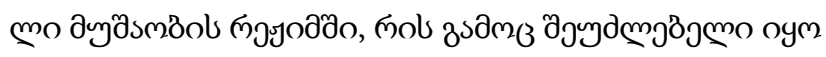

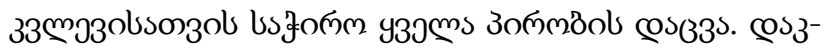




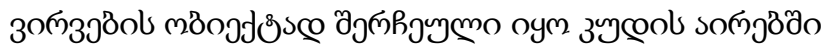

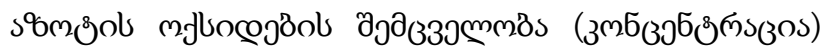

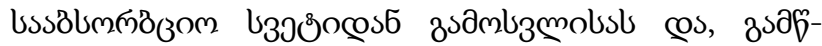

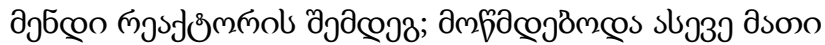

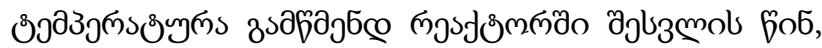

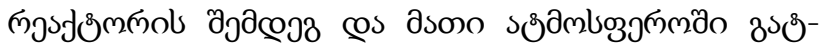

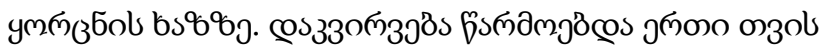

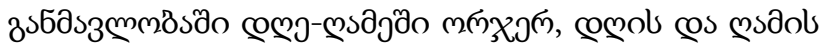

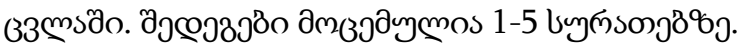

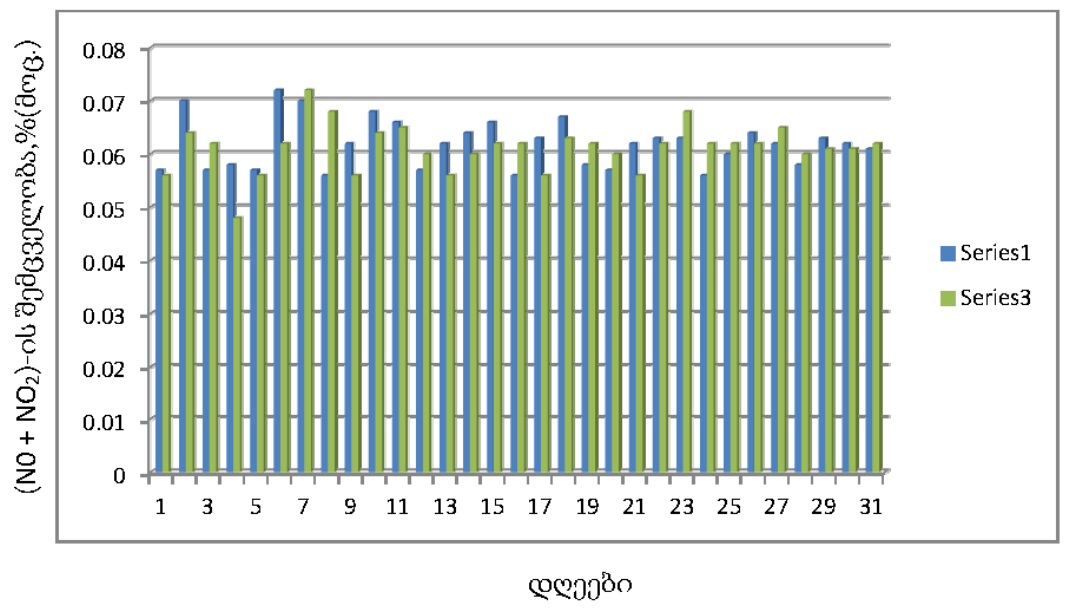

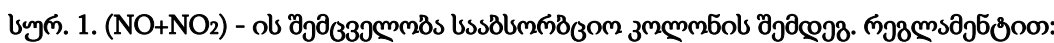
s/y 0,1\% (,1“-

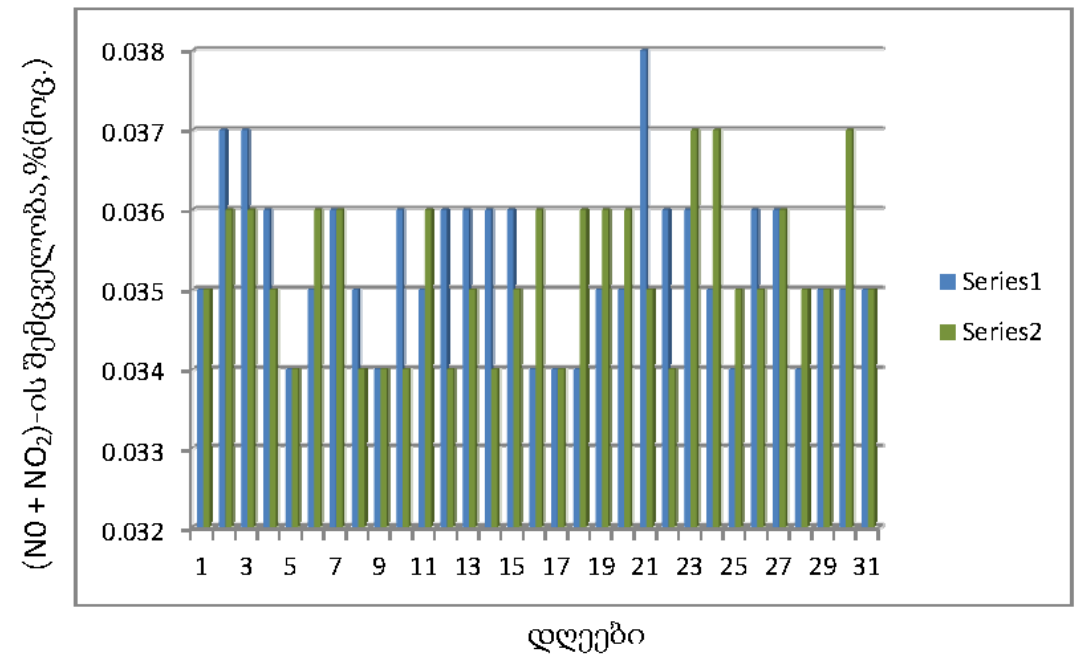

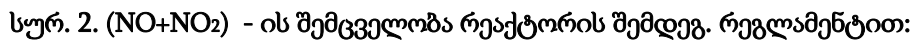

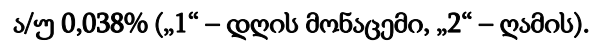




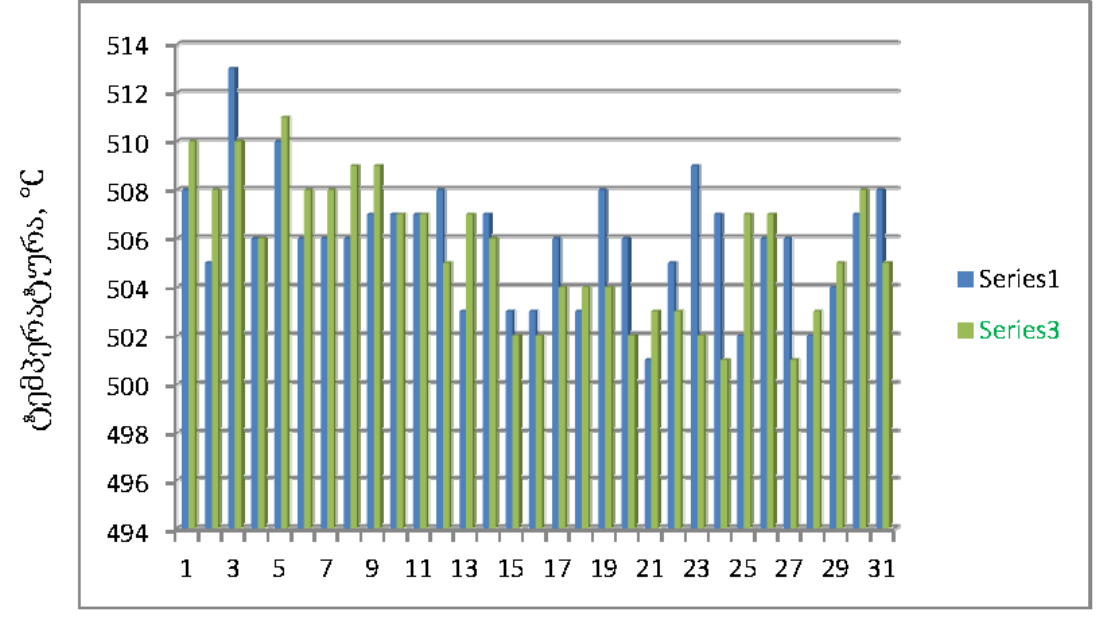

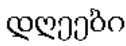

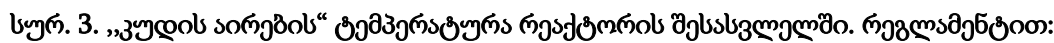

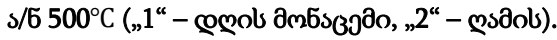

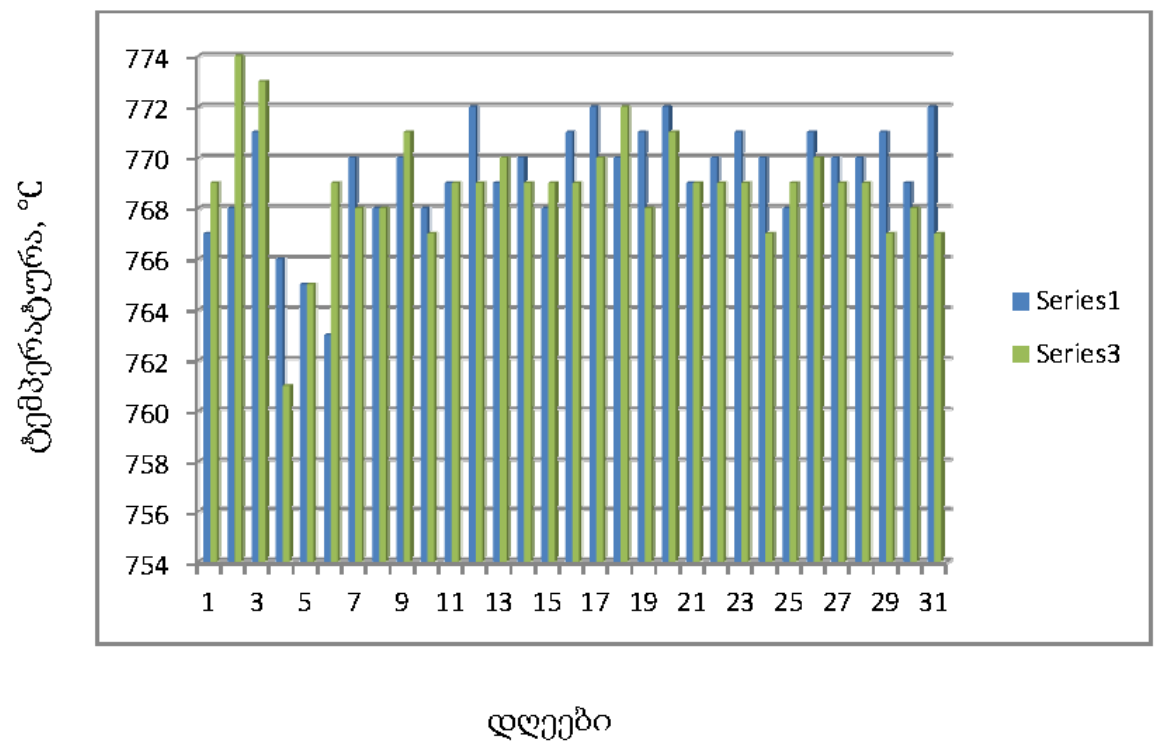

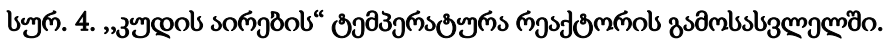

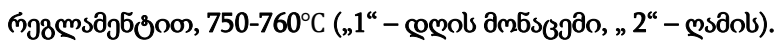




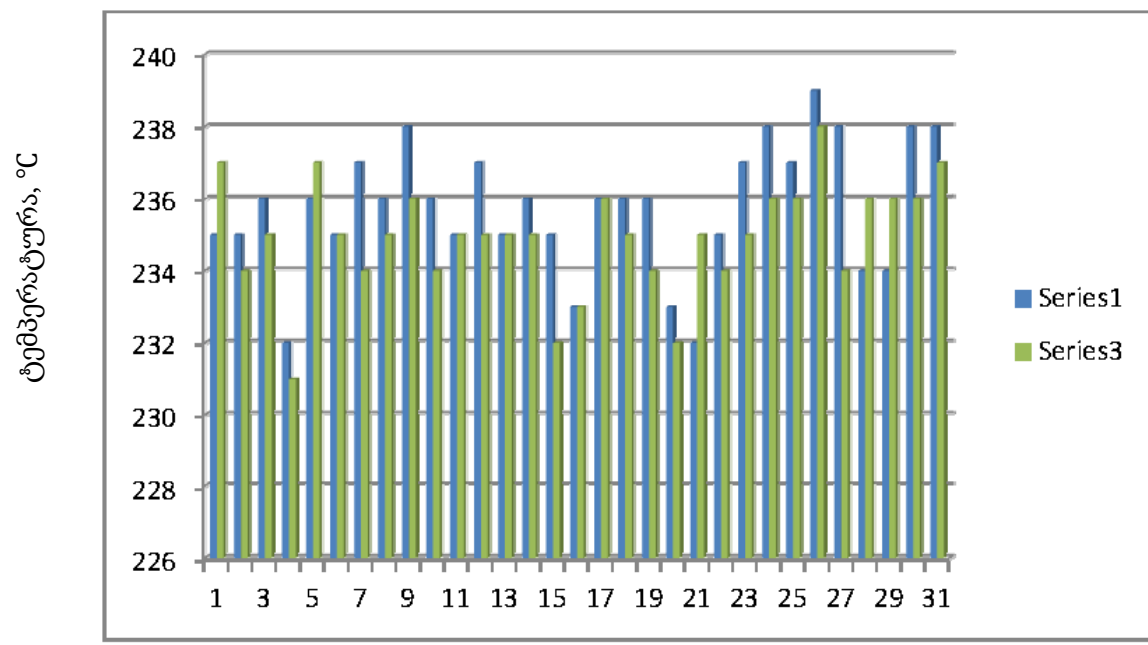

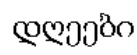

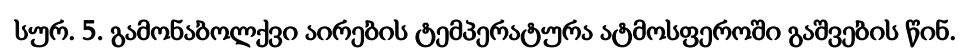

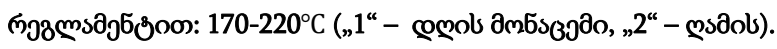

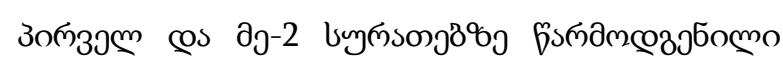

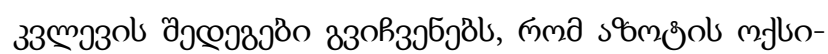

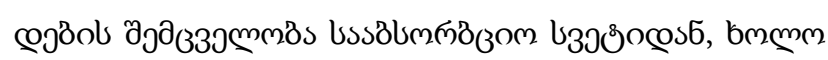

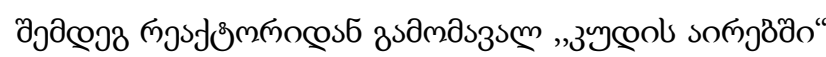

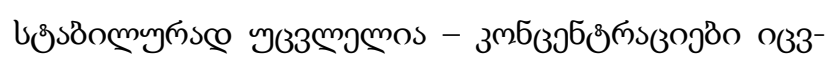

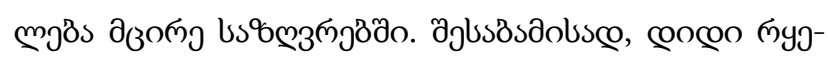

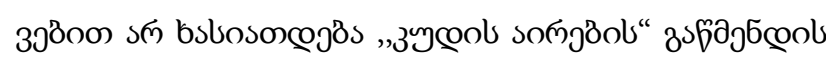

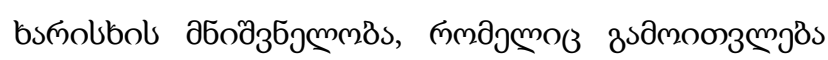
взпдаумоо:

$$
\eta=\frac{\left(\mathrm{C}_{1} \mathrm{~V}_{1}-\mathrm{C}_{2} \mathrm{~V}_{2}\right)}{\mathrm{C}_{1} \mathrm{~V}_{1}} \cdot 100,
$$

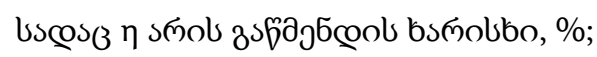

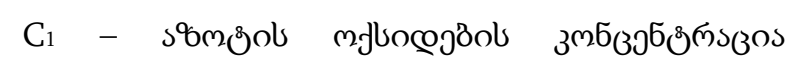

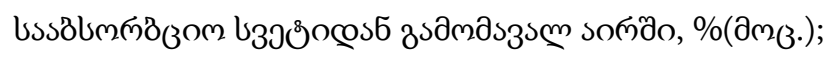

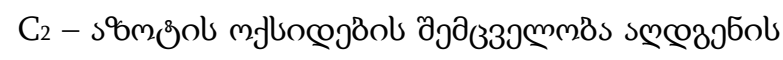

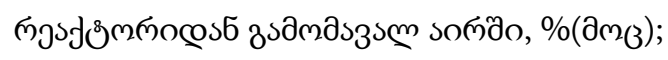

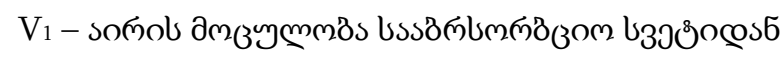

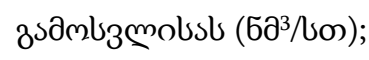

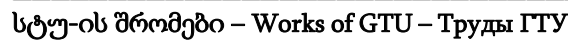
№3 (513), 2019

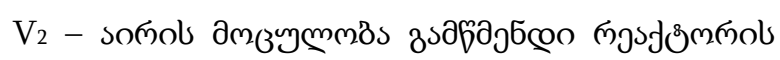

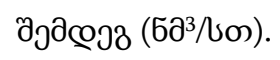

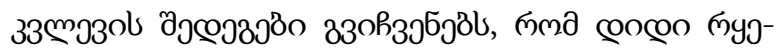

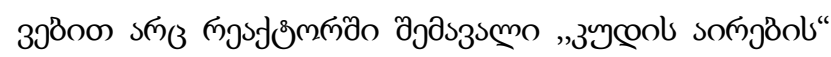

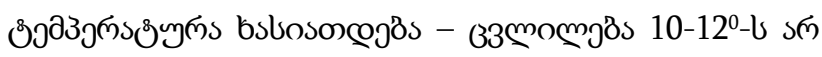

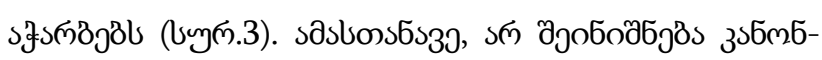

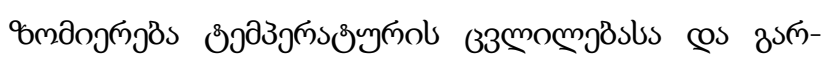

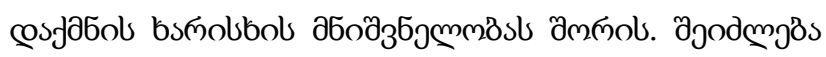
опু3

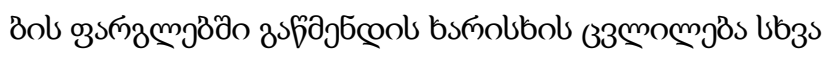

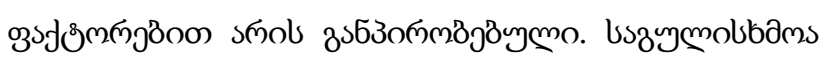

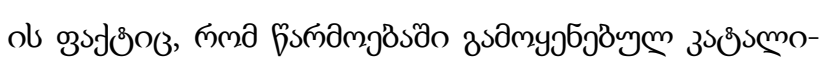

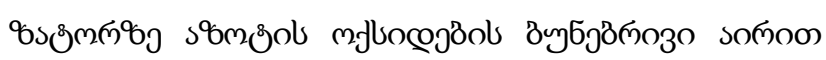

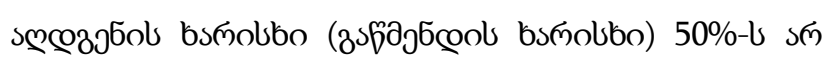

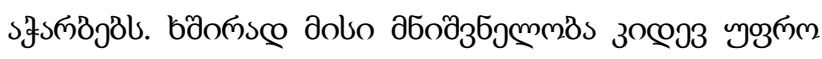

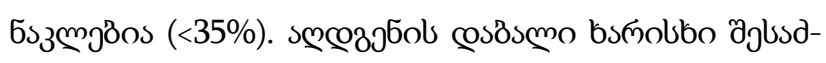

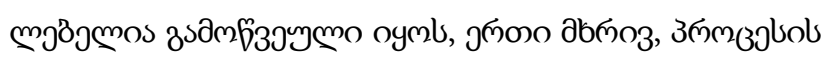

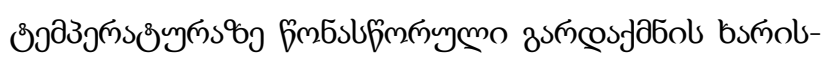

ISSN 1512-0996 www.shromebi.gtu.ge 


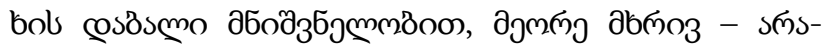

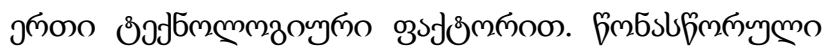

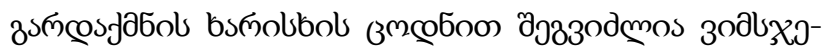

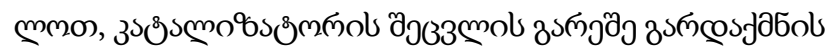

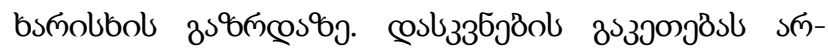

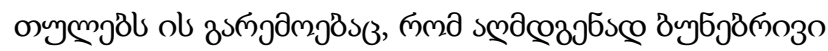

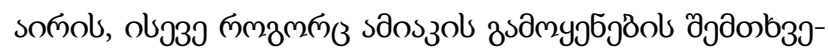

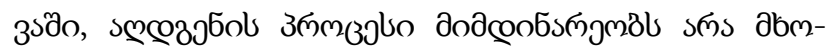

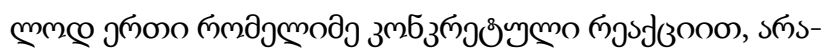

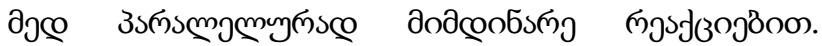

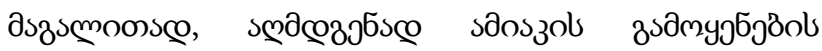

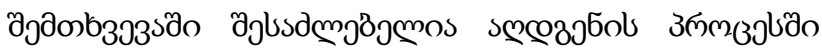

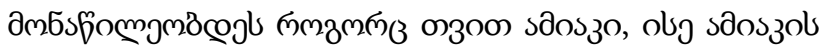

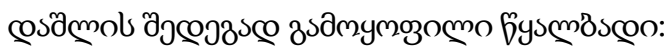

$$
\begin{gathered}
2 \mathrm{NH}_{3}+3 \mathrm{NO} \rightarrow 5 / 2 \mathrm{~N}_{2}+3 \mathrm{H}_{2} \mathrm{O} \\
4 \mathrm{NH}_{3}+3 \mathrm{NO}_{2} \rightarrow 7 / 2 \mathrm{~N}_{2}+6 \mathrm{H}_{2} \mathrm{O} \\
2 \mathrm{H}_{2}+2 \mathrm{NO} \rightarrow \mathrm{N}_{2}+2 \mathrm{H}_{2} \mathrm{O} \\
4 \mathrm{H}_{2}+2 \mathrm{NO}_{2} \rightarrow \mathrm{N}_{2}+4 \mathrm{H}_{2} \mathrm{O}
\end{gathered}
$$

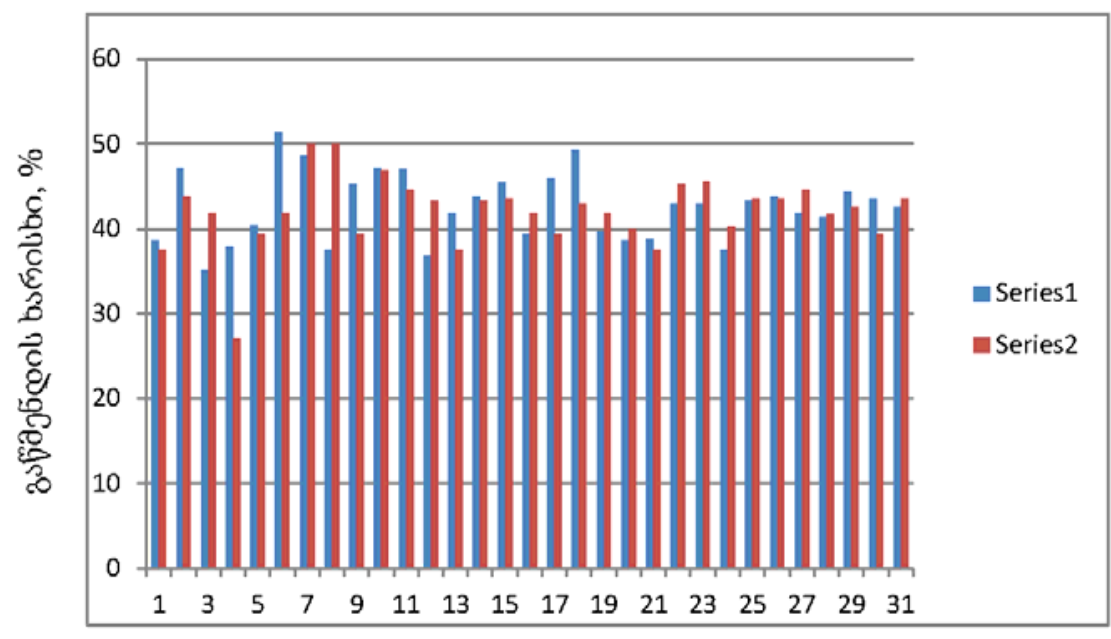

esjojo

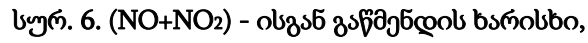

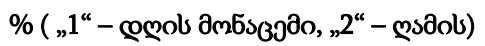

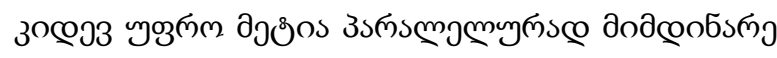

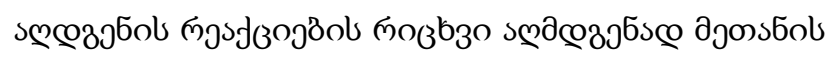

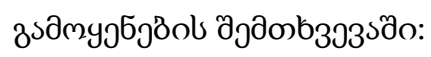

$$
\begin{gathered}
\mathrm{CH}_{4}+4 \mathrm{NO} \rightarrow \mathrm{CO}_{2}+2 \mathrm{~N}_{2}+2 \mathrm{H}_{2} \mathrm{O} \\
\mathrm{CH}_{4}+2 \mathrm{NO}_{2} \rightarrow \mathrm{CO}_{2}+\mathrm{N}_{2}+2 \mathrm{H}_{2} \mathrm{O} \\
\mathrm{CO}+\mathrm{NO} \rightarrow \mathrm{CO}_{2}+\frac{1}{2}{ }_{2} \mathrm{~N}_{2} \\
2 \mathrm{CO}+2 \mathrm{NO} \rightarrow 2 \mathrm{CO}_{2}+\mathrm{N}_{2} \\
2 \mathrm{H}_{2}+2 \mathrm{NO} \rightarrow \mathrm{N}_{2}+2 \mathrm{H}_{2} \mathrm{O}
\end{gathered}
$$

$$
\begin{gathered}
4 \mathrm{H}_{2}+2 \mathrm{NO}_{2} \rightarrow \mathrm{N}_{2}+4 \mathrm{H}_{2} \mathrm{O} \\
2 \mathrm{CH}_{4}+6 \mathrm{NO} \rightarrow 2 \mathrm{CO}+3 \mathrm{~N}_{2}+4 \mathrm{H}_{2} \mathrm{O} \\
2 \mathrm{CH}_{4}+3 \mathrm{NO}_{2} \rightarrow 2 \mathrm{CO}+3 / 2{ }_{2} \mathrm{~N}_{2}+4 \mathrm{H}_{2} \mathrm{O}
\end{gathered}
$$

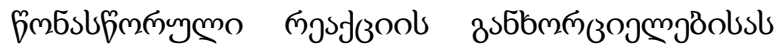

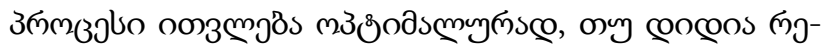

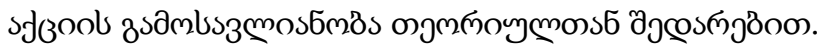

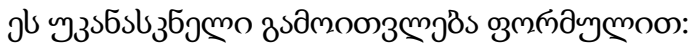

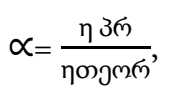




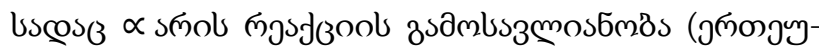
mo fomo);

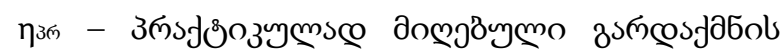
bsnolbo;

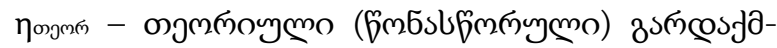

bol bśnolbo;

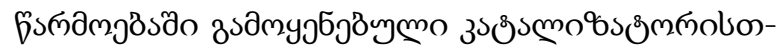

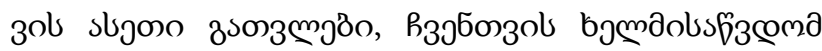

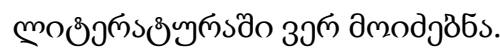

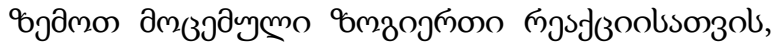

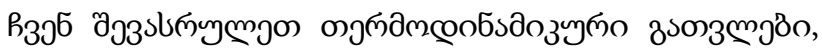

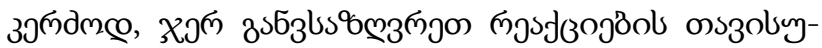

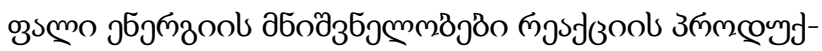

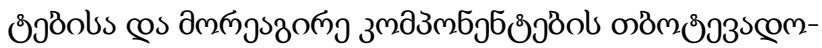

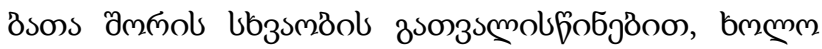

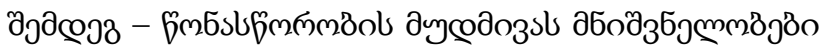

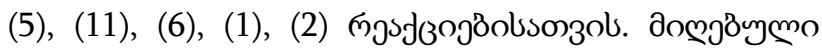

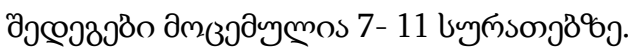

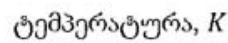

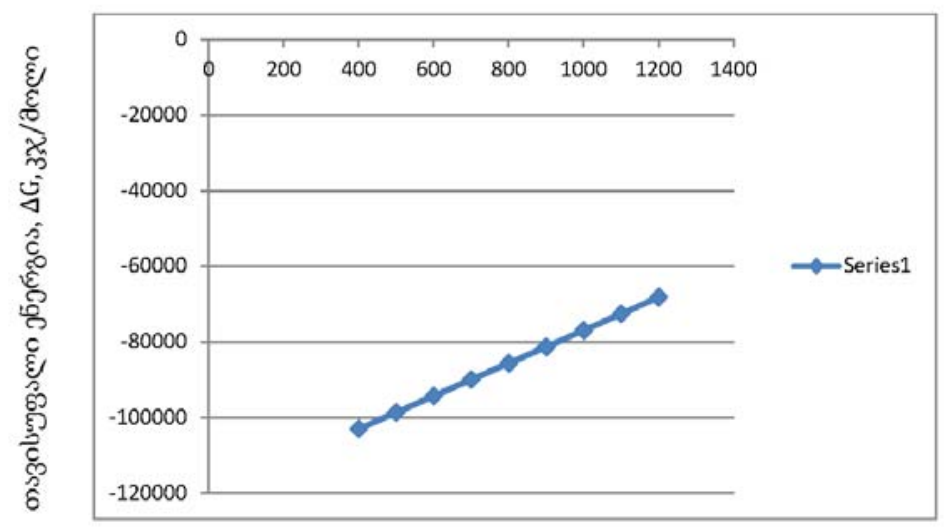

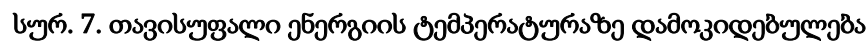

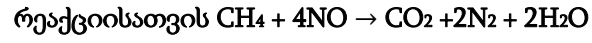

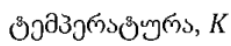

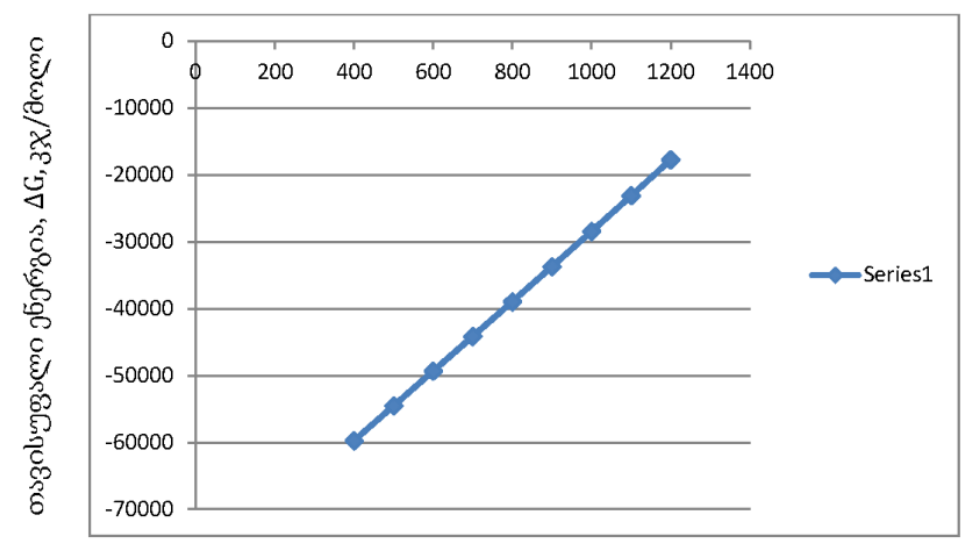

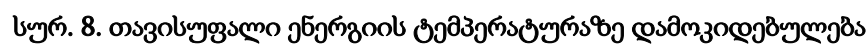

ngsfloobson 30 : $2 \mathrm{CH}_{4}+6 \mathrm{NO} \rightarrow 2 \mathrm{CO}+3 \mathrm{~N}_{2}+4 \mathrm{H}_{2} \mathrm{O}$ 


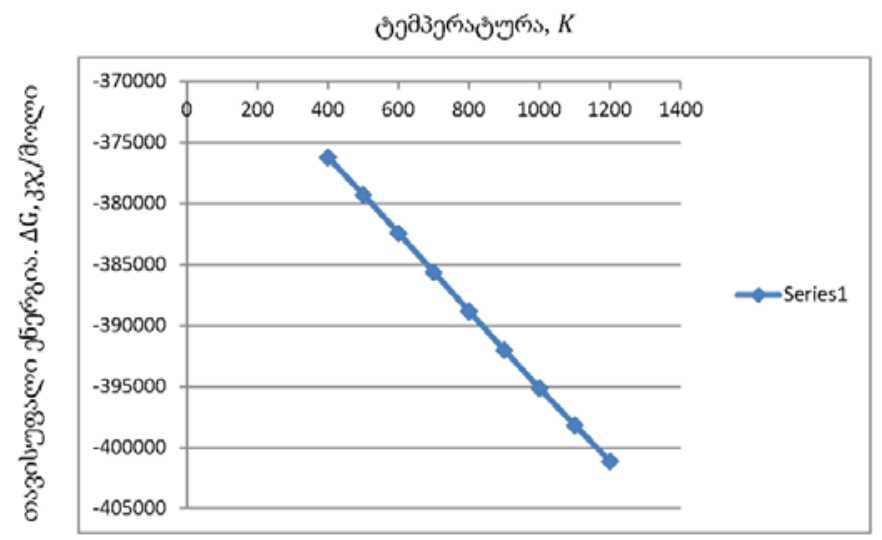

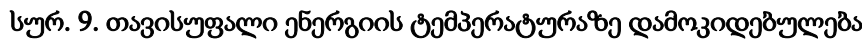
r.jstionssorsol: $\mathrm{CH}_{4}+2 \mathrm{NO}_{2} \rightarrow \mathrm{CO}_{2}+\mathrm{N}_{2}+2 \mathrm{H}_{2} \mathrm{O}$

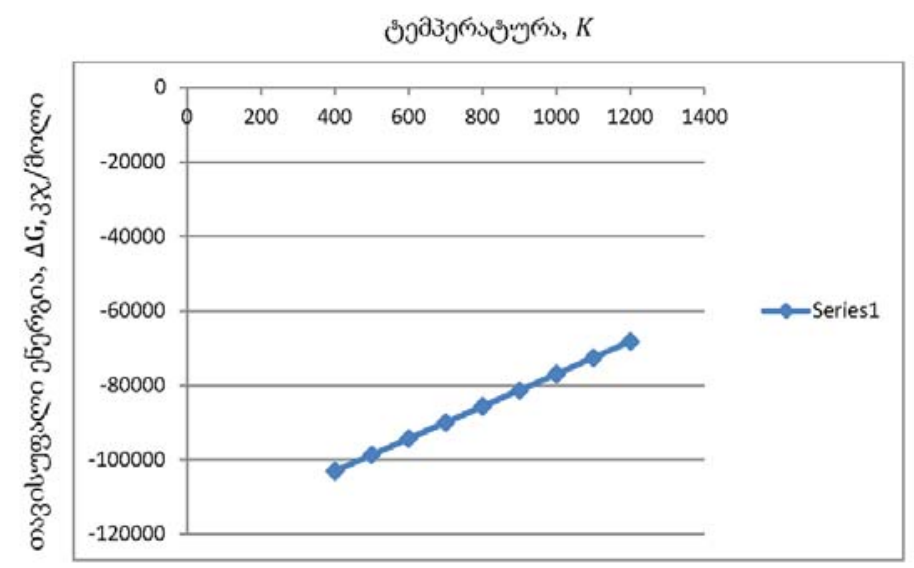

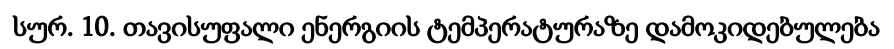

mastooobson 3ob: $4 \mathrm{NH}_{3}+6 \mathrm{NO} \rightarrow 5 \mathrm{~N}_{2}+6 \mathrm{H}_{2} \mathrm{O}$

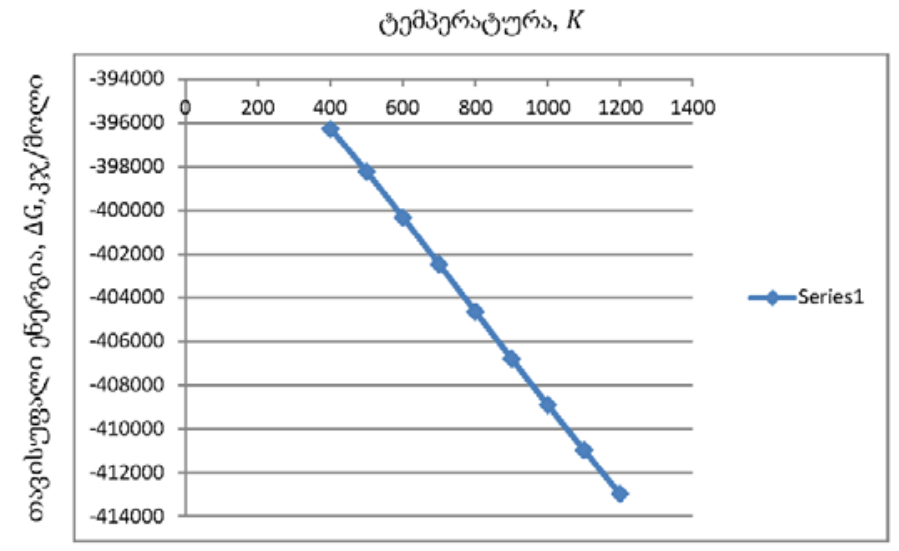

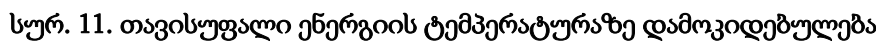
most3oobsom 30 : $8 \mathrm{NH}_{3}+6 \mathrm{NO}_{2} \rightarrow 7 \mathrm{~N}_{2}+12 \mathrm{H}_{2} \mathrm{O}$ 


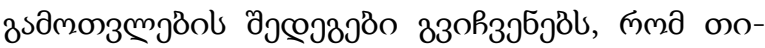

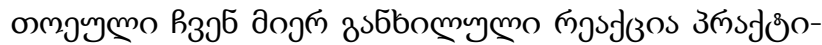

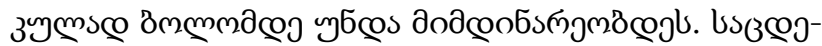

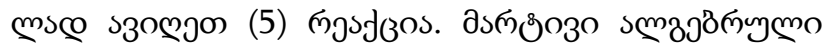

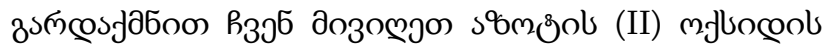

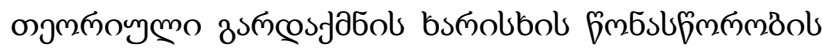

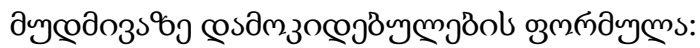

$$
x=\frac{1,741 \times K_{p}^{0.8}}{1+1,741 \times K_{p}^{0.8}},
$$

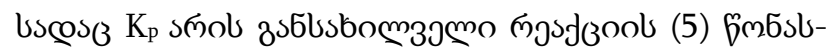

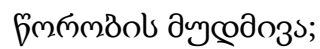

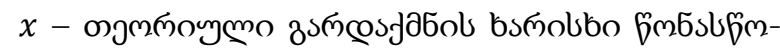

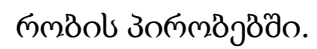

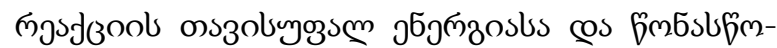

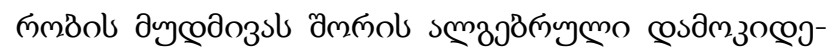

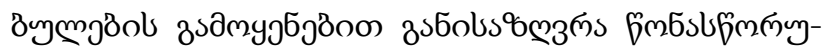

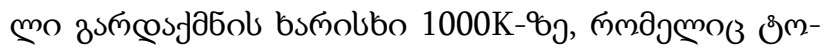
мо sмдмßбесs 0,9997-ob sбy 99,97\%- ob.

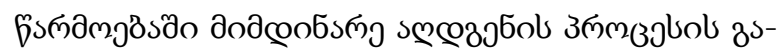

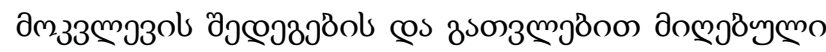

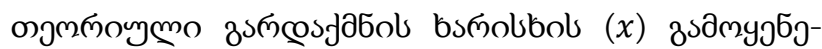

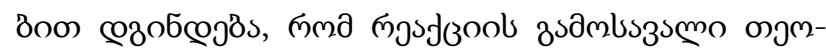

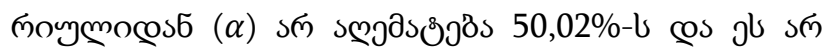

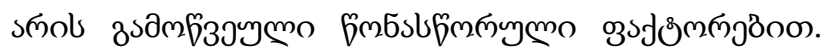

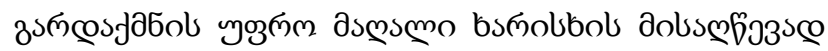

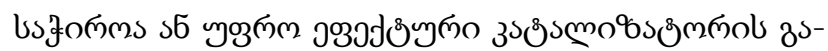

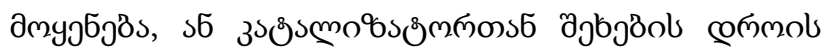

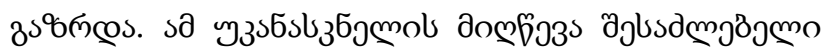

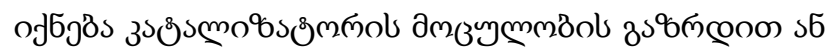

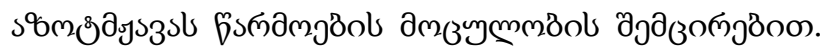

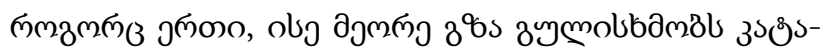

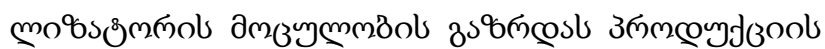

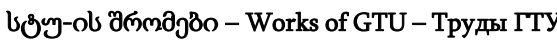

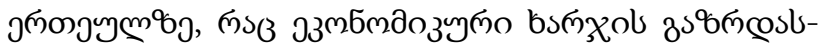

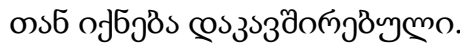

\section{costs335s}

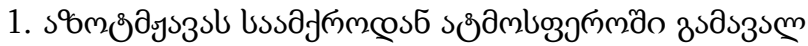

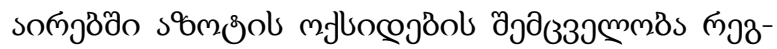

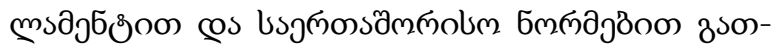

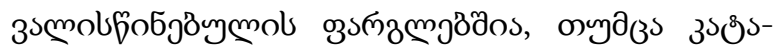

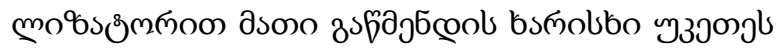

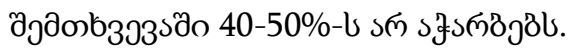

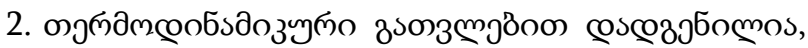

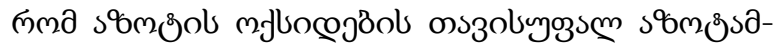

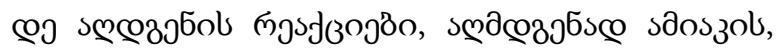

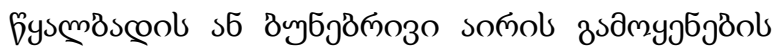

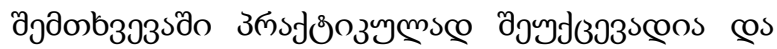

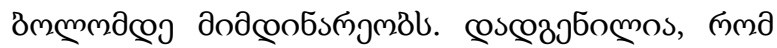

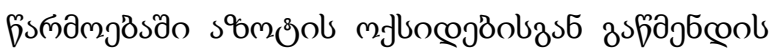

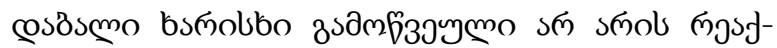

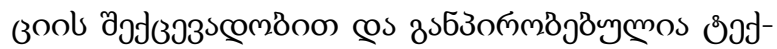

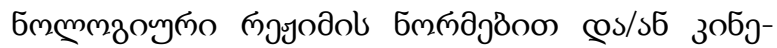

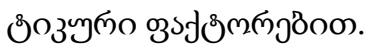

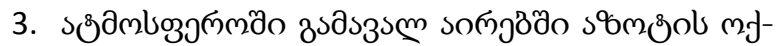

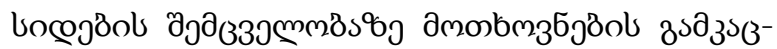

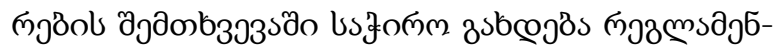

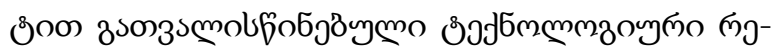

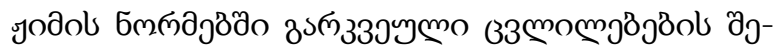

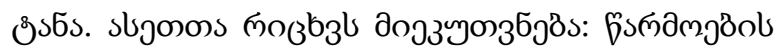

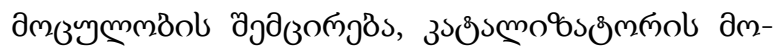

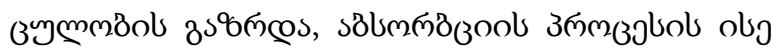

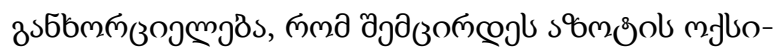

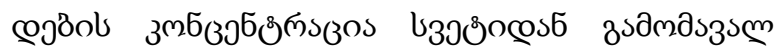

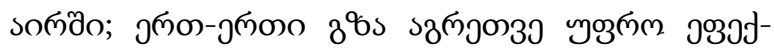

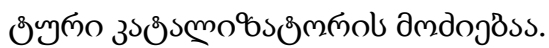

ISSN 1512-0996 www.shromebi.gtu.ge 


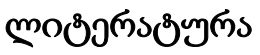

1. Weks E. U. Blok F. Ye. Thermodynamic properties of 65 elements--their oxides, halides, carbides, and nitrides. M.: „Metallurgy”. 1965, 145 p. (in Russian).

2. Ryabin V.A., Ostroumov M.A., Sweet T.F. Yakhontova E. L., Petropavlovsky I. A. Thermodynamic properties of substances. L.: "Khimiya". 1977, 392 p. (in Russian).

3. Zagoruchenko V.A., Zhuravlev A.M. Thermodynamic properties of gaseous and liquid methane. M.: "Izdatelstvo standartov". 1969, 235 p. (in Russian).

4. Handbook of Nitrogen. Vol.2. M.: "Khimiya”. 1969. 445 p. (in Russian).

5. Nitric acid production in large-scale single units. Edited by V.M. Olevsky. M.: "Khimiya". 1985, 400 p. (in Russian).

6. Veriatan W.D. Thermodynamic properties of inorganic substances. Handbook. M.: "Atomizdat". 1965, 112 p. (in Russian).

7. Method for catalytic purification of waste gases from nitrogen oxides. Patent for invention N2174430. 10.10.2001. (in Russian).

8. Karavaev M.M., Zasorin A.P., Kleshchev N.F. Catalytic Oxidation of Ammonia. M.: "Khimiya “. 1983, 232 p. (in Russian). 
UDC 66.074.32

SCOPUS CODE 2310

DOI: https://doi.org/10.36073/1512-0996-2019-3-86-97

\title{
Research of the process of exhaust gas cleaning in the production of nitric acid from nitrogen oxides
}

\author{
Marlen Mchedlishvili Department of Chemical Technology and Biotechnology, Georgian Technical \\ University, 69 M. Kostava str, 0160 Tbilisi, Georgia \\ E-mail: m.mchedlishvili@gtu.ge \\ Aleksandre Aphakidze Rustavi JSC "Azoti", Peace 2, 3702 Rustavi, Georgia \\ E-mail: alika.aphak@gmail.com
}

\section{Reviewers:}

J. Shengelia, Professor, Faculty of Chemical Technology and Metallurgy, GTU

E-mail: jemal.shengelia@gtu.ge

I. Bazgadze, Associate Professor, Faculty of Chemical Technology and Metallurgy, GTU

E-mail: i.bazgadze@gtu.ge

Abstract. The process of exhaust gas cleaning in the production of Nitric acid from Nitrogen oxides (NO + $\mathrm{NO}_{2}$ ) was studied by catalytic reduction of natural gases. It has been established that the content of Nitrogen oxides in gases leaving the catalytic reactor is low, however it complies with the production regulations and does not exceed the maximum permissible concentrations (MPC) standards adopted by the relevant international organizations, the catalyst activity is low - the purification rate does not exceed $50 \%$.

At the same time, thermodynamic analysis showed that the theoretical extent of gas purification from Nitrogen oxides, at the temperature of the catalytic reduction of natural gas production process, is $99.97 \%$.

Within the strict requirements of the relevant organizations for the content of Nitrogen oxides in the exhaust gases of Nitric acid production, there are two main solutions available to increase the degree of gas purification: 1) providing appropriate changes in process regulations and 2) using more efficient catalyst for exhaust gas cleaning from nitrogen oxides.

Resume:

Key words: Catalyst; cleaning; Nitric acid; Nitrogen oxides; thermodynamic analysis. 
UDC 66.074.32

SCOPUS CODE 2310

DOI: https://doi.org/10.36073/1512-0996-2019-3-86-97

\title{
Исследование процесса очистки отходящих газов производства азотной кислоты от оксидов азота
}

\author{
Марлен \\ Мчедлишвили \\ Департамент химической технологии и металлургии, Грузинский технический \\ университет, Грузия, 0160, Тбилиси, ул. М. Костава, 69 \\ E-mail: m.mchedlishvili@gtu.ge \\ Александр Апакидзе \\ Руставское A/O «Азот», Грузия, 3702, Рустави, ул. Мшвидоба 2 \\ E-mail: alika.aphak@gmail.com
}

\section{Рецензенты:}

Д. Шенгелия, профессор факультета химической технологии и металлургии ГТУ

E-mail: jemal.shengelia@gtu.ge

И. Базгадзе, профессор факультета химической технологии и металлургии ГТУ

E-mail: i.bazgadze@gtu.ge

Аннотация. Изучен процесс очистки выхлопных газов в производстве азотной кислоты от оксидов азота (NO+NO2) путем каталитического восстановления природным газом. Установлено, что хотя содержание оксидов азота в выходящих из каталитического реактора газах, небольшое, соответствует регламенту производства и не превышает норм П.Д.К., принятых соответствующими международными организациями, активность катализатора низкая, - степень очистки не превышает 50\%.

При этом термодинамический анализ показал, что теоретическая степень очистки газа от оксидов азота, при температуре производственного процесса каталитического восстановления оксидов азота природным газом составляет 99,97\%.

При ужесточении требований соответствующими организациями на содержание оксидов азота в выхлопных газах производства азотной кислоты, имеются два решения проблемы повышения степени очистки газа: 1) внесение изменений в технологический регламент и 2) использование более эффективного катализатора для очистка газов от оксидов азота.

Ключевые слова: азотная кислота; катализатор; оксиды азота; очистка; термодинамический анализ.

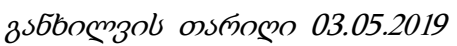

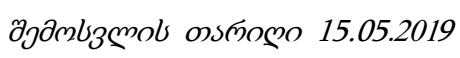

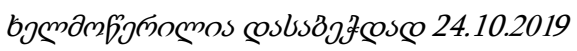


UDC 669:187.526.001.5

SCOPUS CODE 2501

DOI: https://doi.org/10.36073/1512-0996-2019-3-98-110

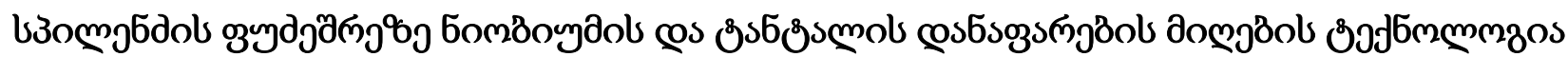

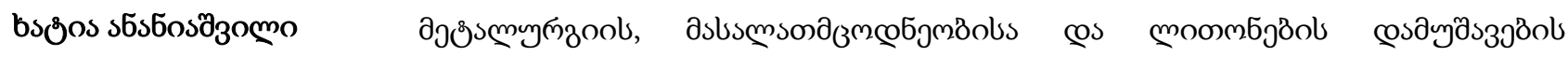

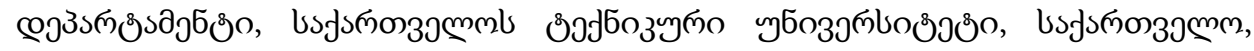

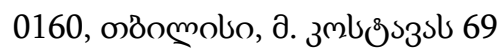

E-mail: kh.ananiashvili@gtu.ge

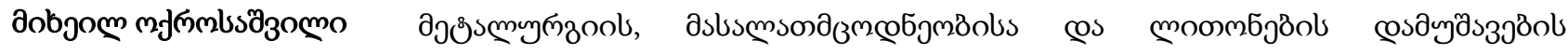

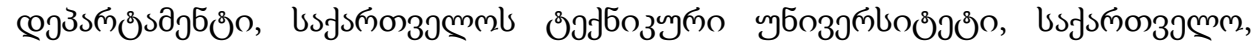

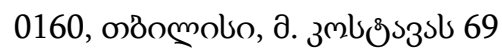

E-mail: m.okrosashvili@gtu.ge

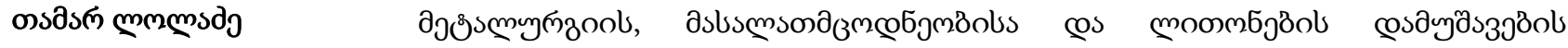

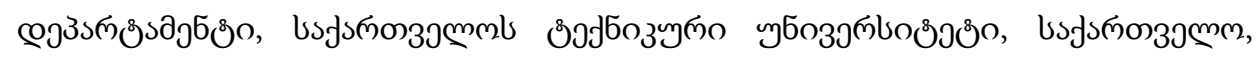

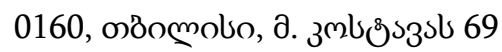

E-mail: t.loladze@gtu.ge

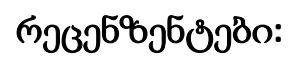

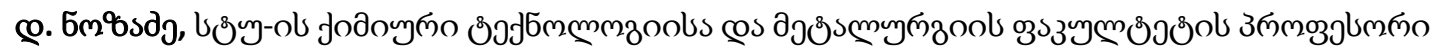

E-mail: d.nozadze@gtu.ge

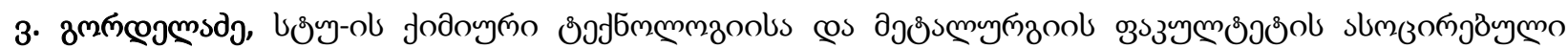

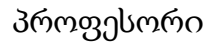

E-mail: v.gordeladze@gtu.ge

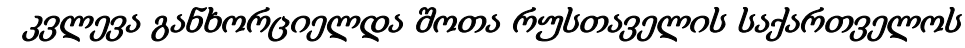

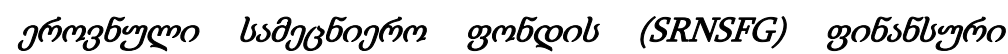

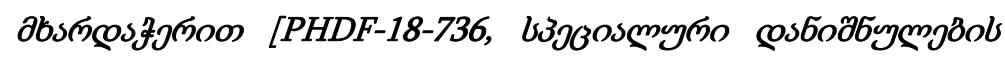

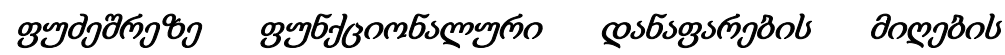

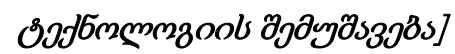

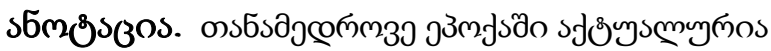

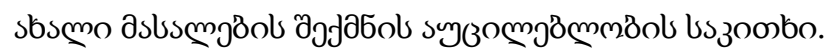

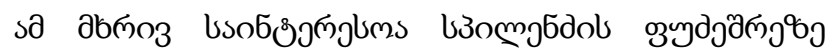

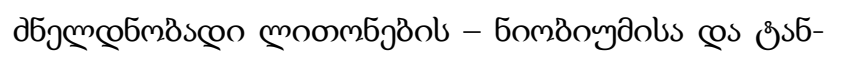

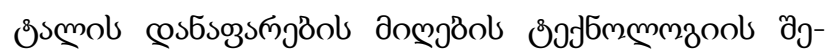

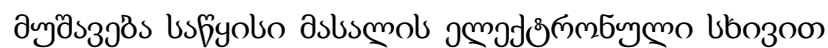

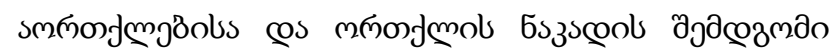

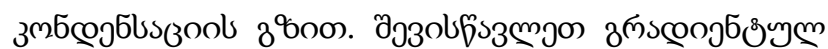

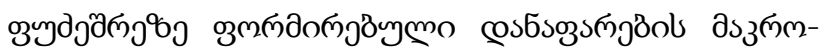




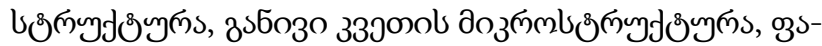

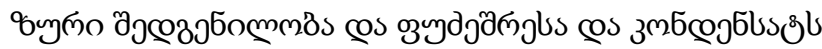

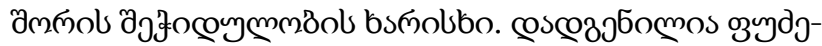

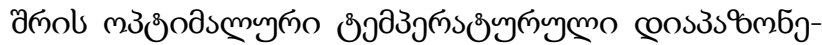

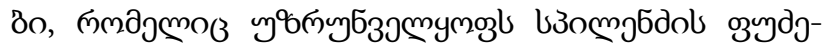

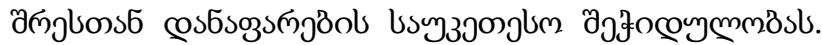

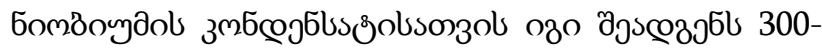

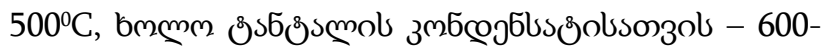

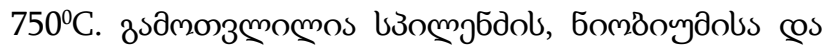

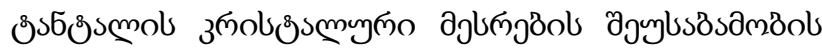

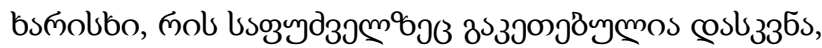

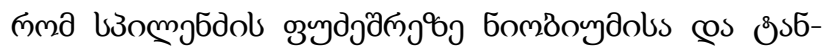

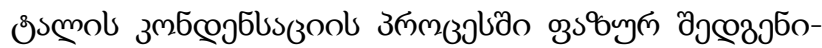

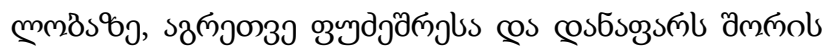

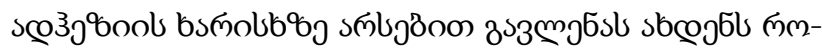

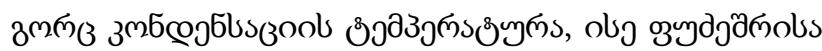

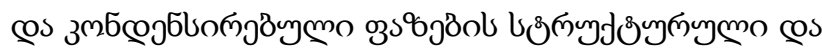

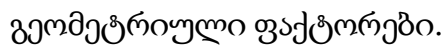

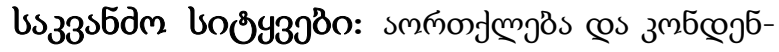

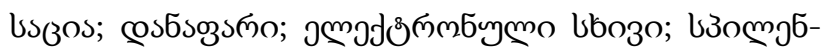

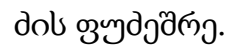

\section{ajlsзssmo}

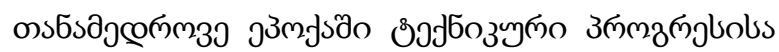

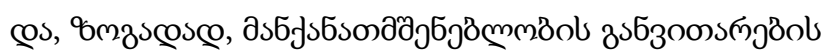

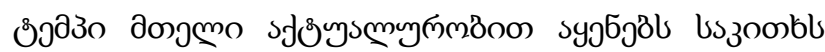

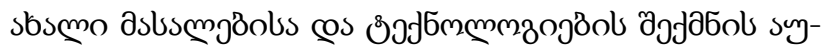

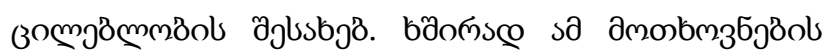

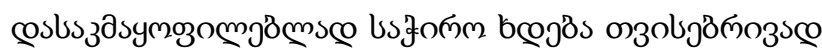

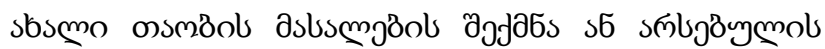

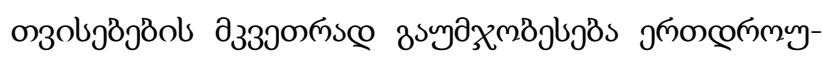

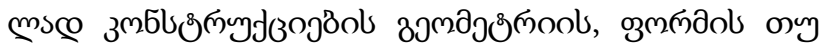

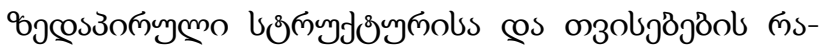

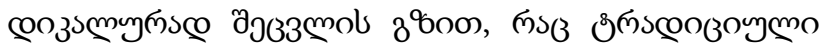

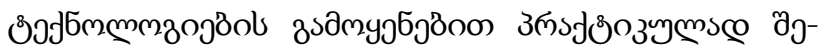

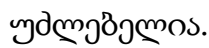

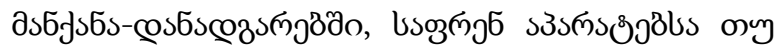

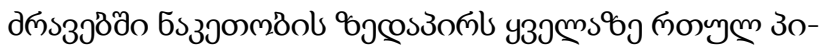

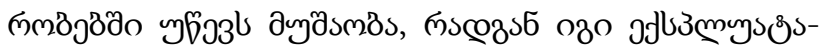

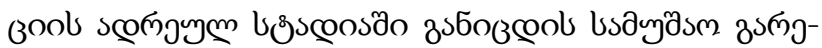

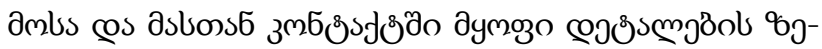

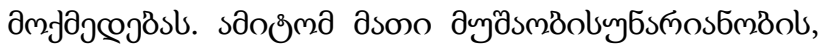

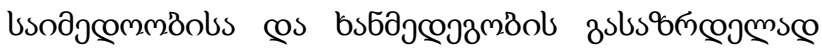

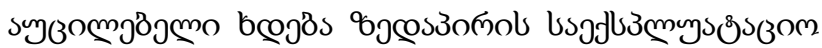

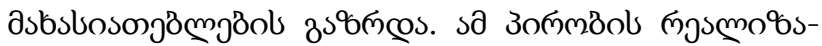

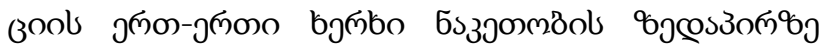

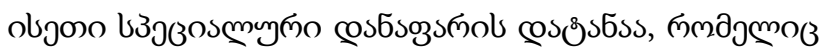

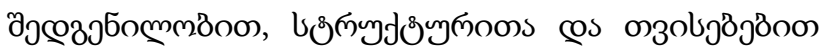

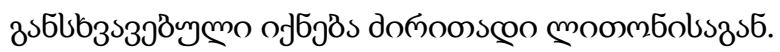

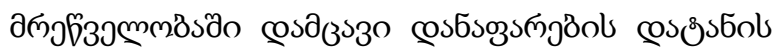

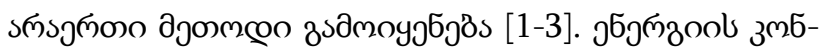

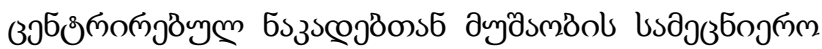

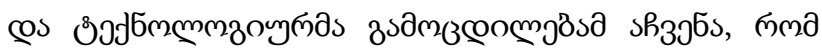

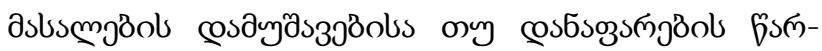

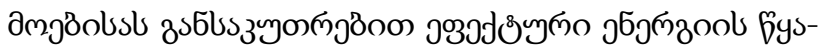

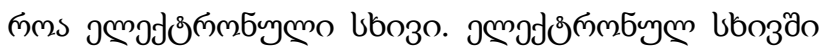

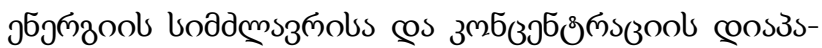

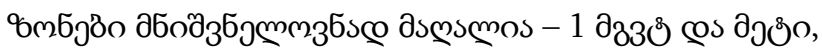

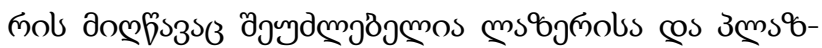

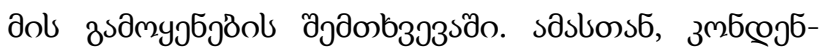

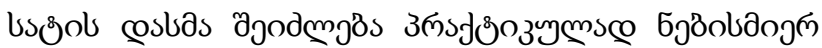

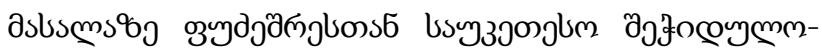

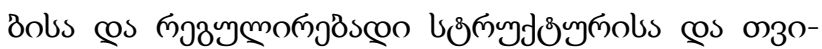

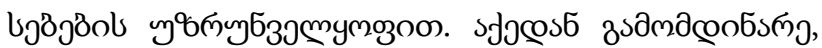




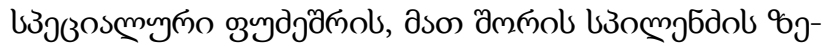

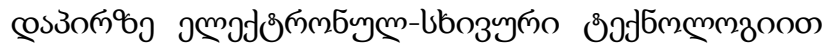

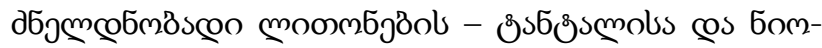

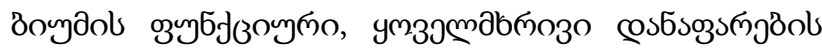

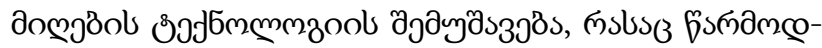

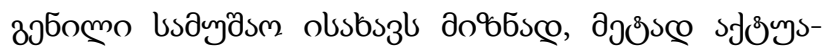

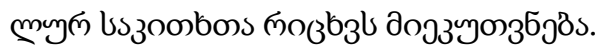

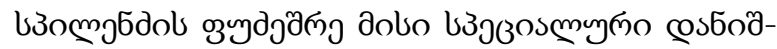

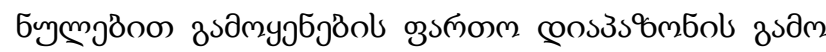

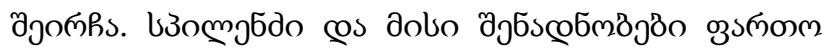

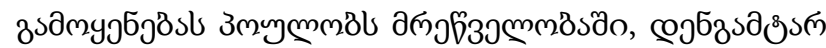

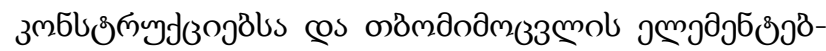

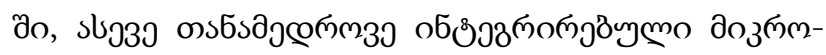

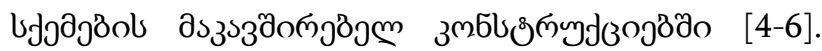

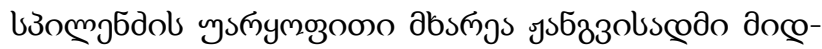

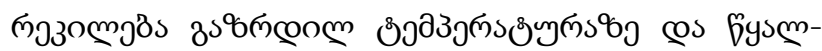

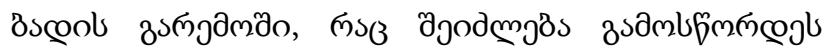

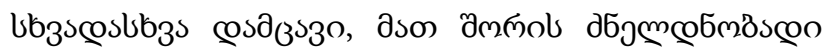

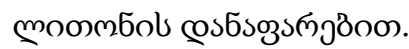

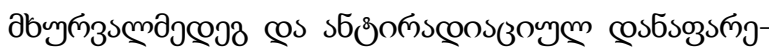

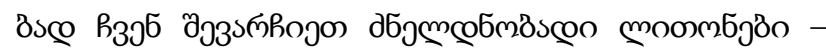

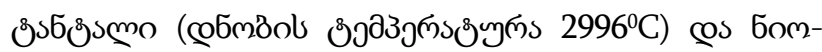

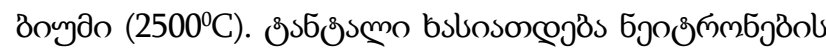

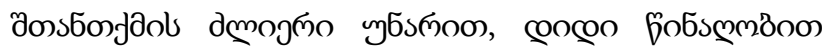

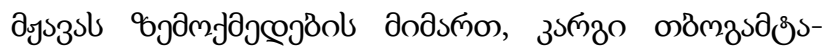

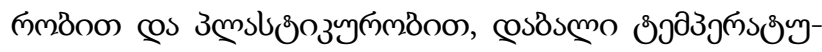

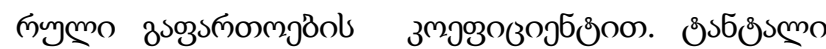

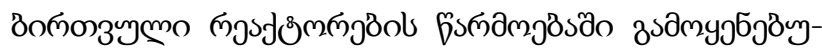

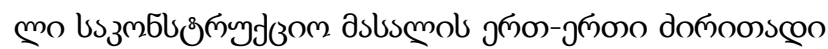

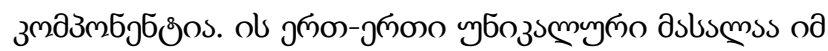

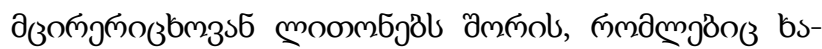

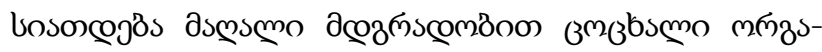

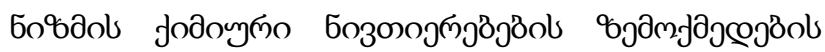

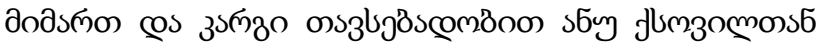

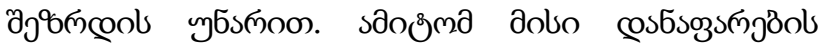

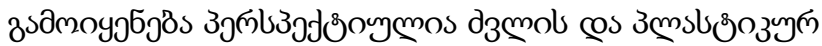

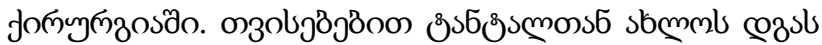

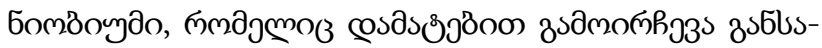

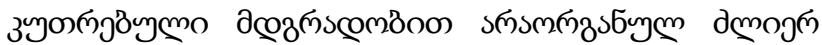

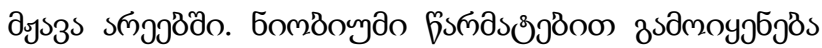

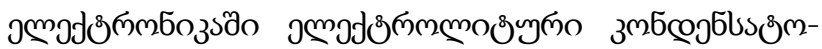

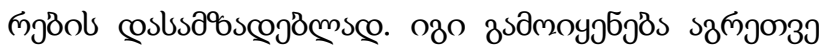

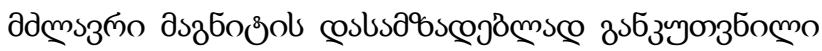

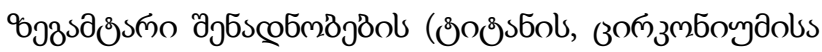

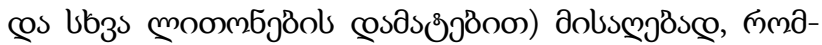

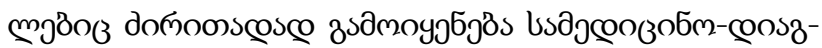

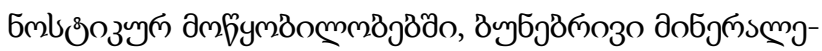

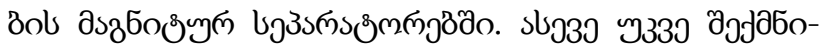

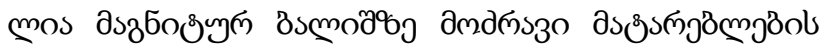
aszolşns

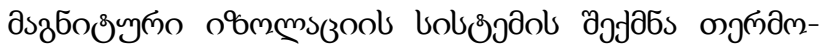

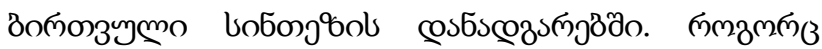

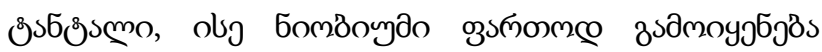

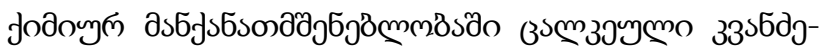

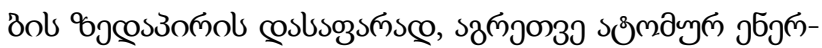

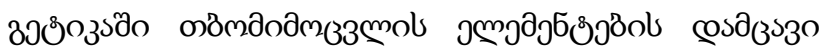

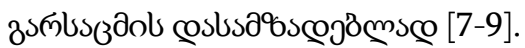

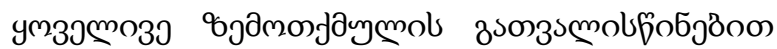

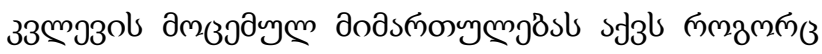

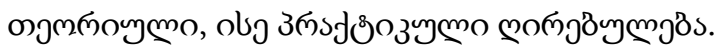

\section{domooscen 5sfomo}

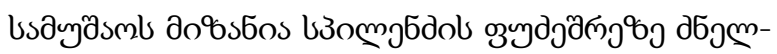

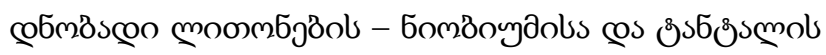

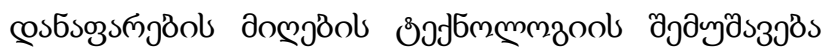

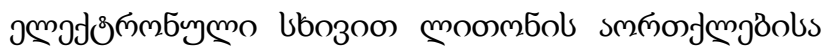




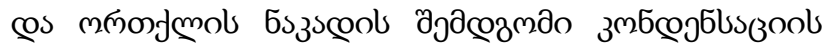

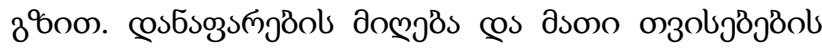

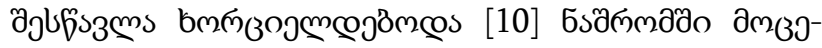
аулмо дуомщозоо.

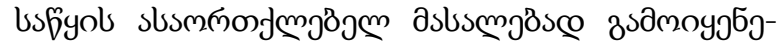

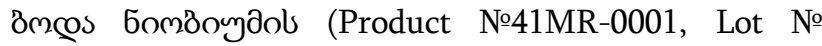

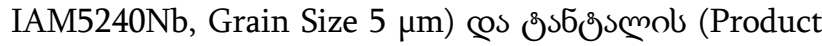
№ 73MR-0001, Lot № IAM1283TAM, Grain Size $5 \mu \mathrm{m}$ )

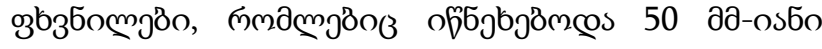

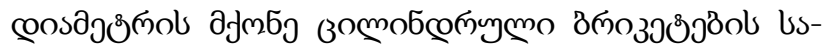

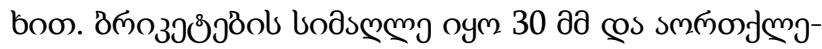

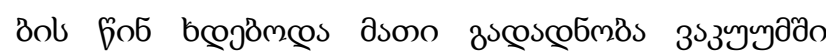

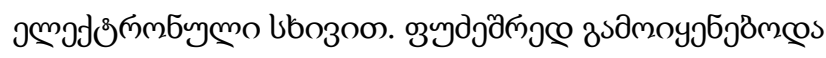

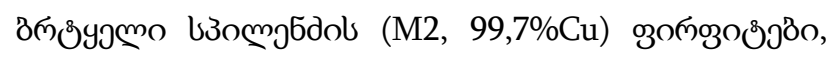

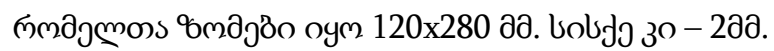

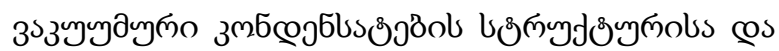

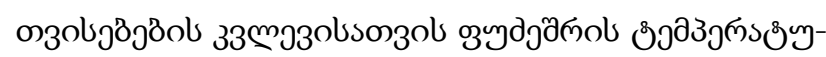

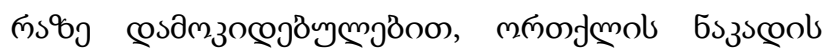

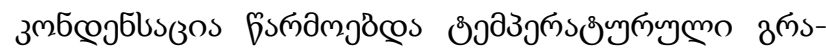

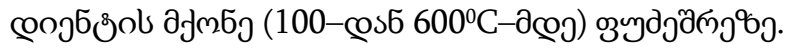
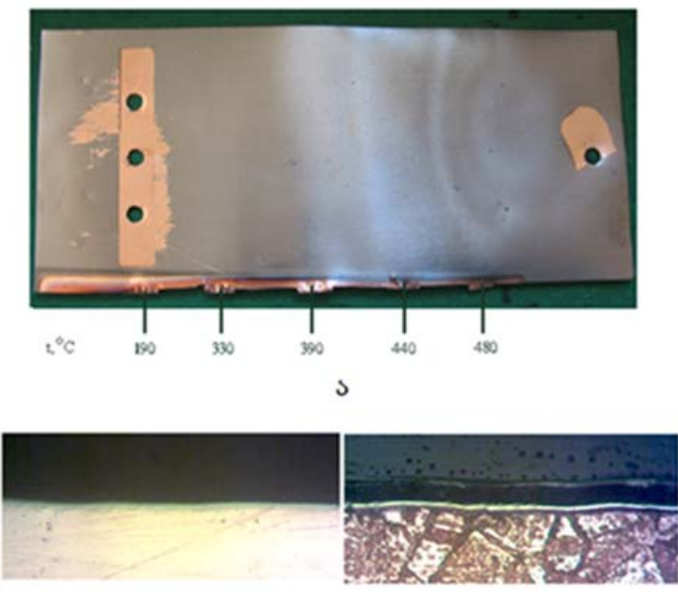

o

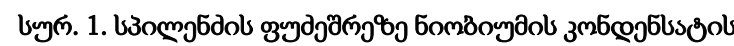

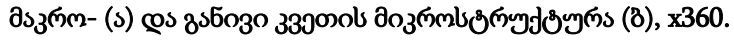

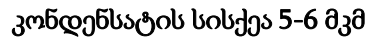

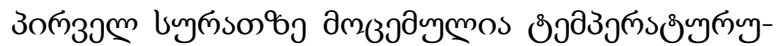

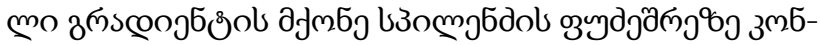

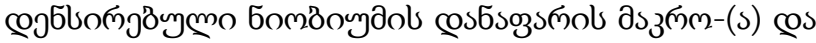

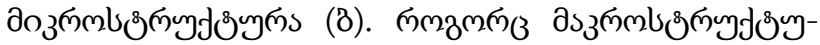

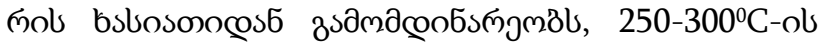

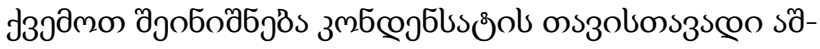

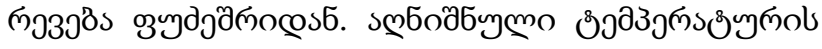

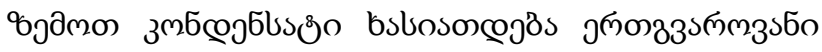

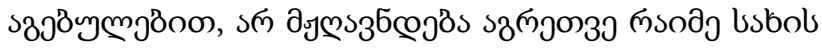

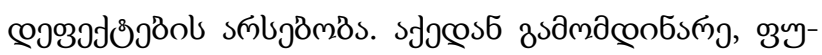

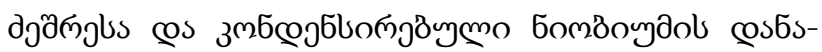

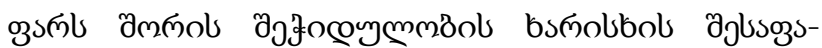

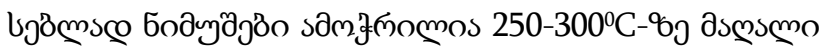

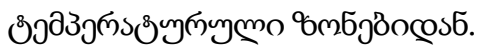

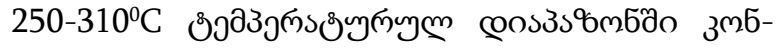

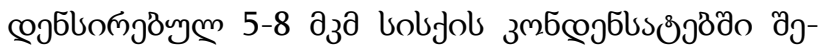

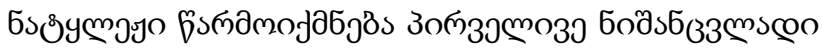

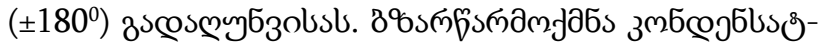

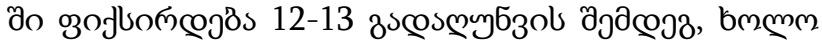

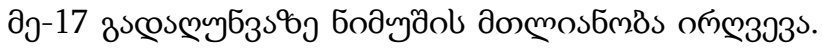

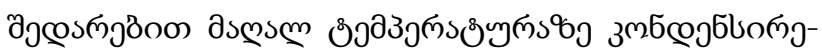

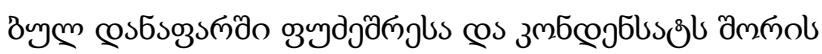

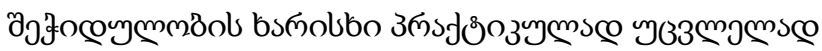

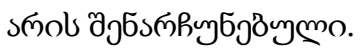

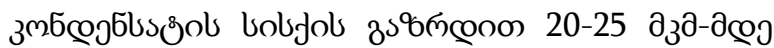

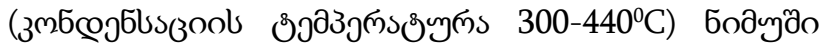

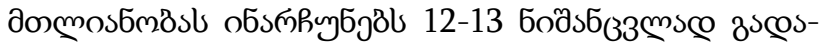

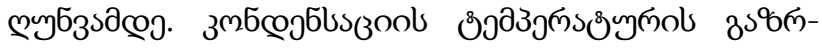

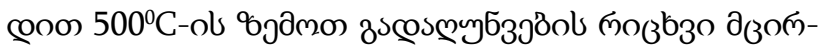

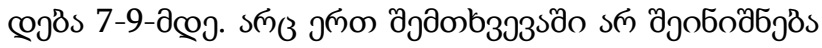

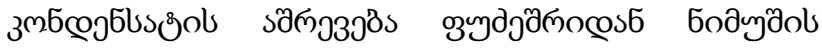

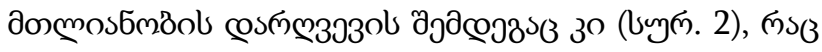

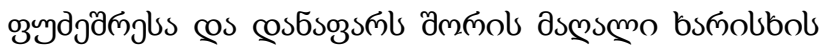

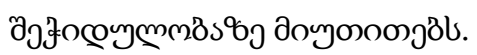




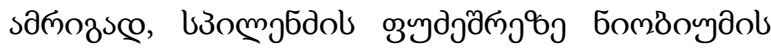

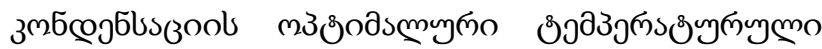

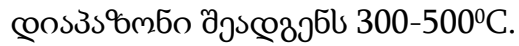

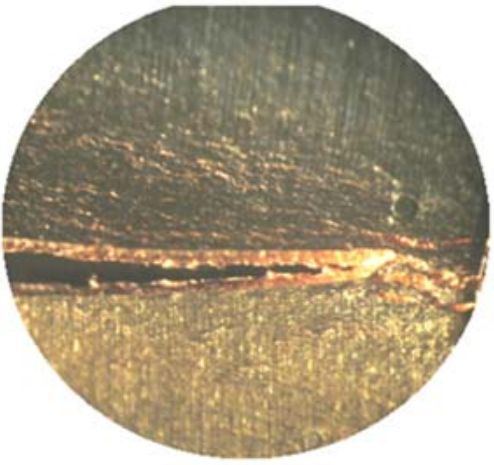

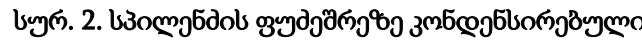

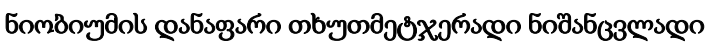

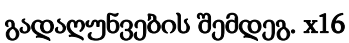

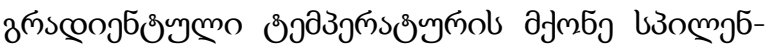

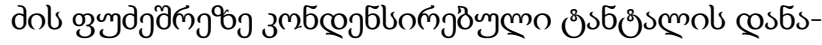

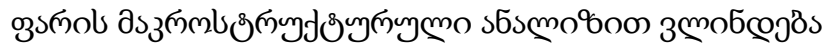

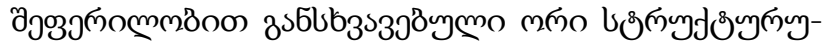

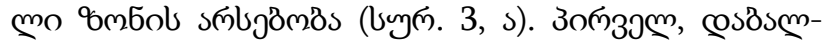

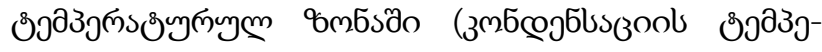

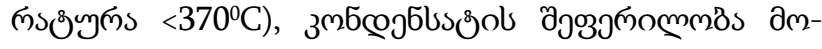

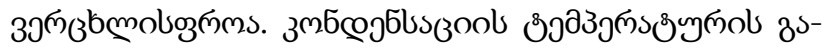

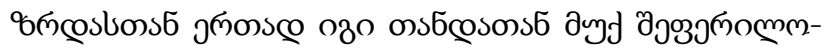

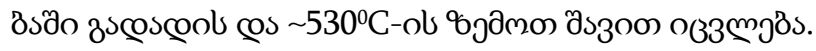

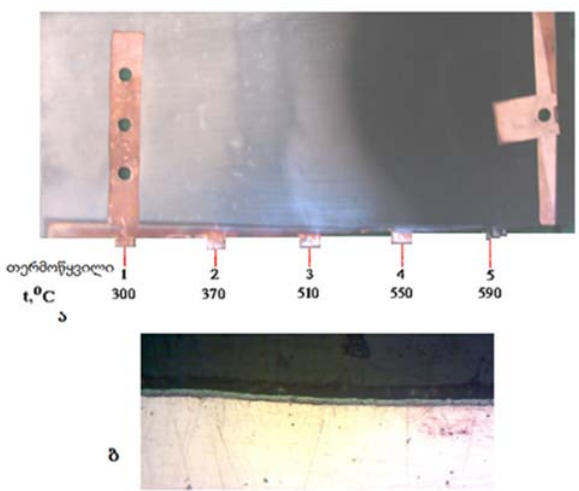

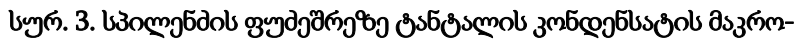

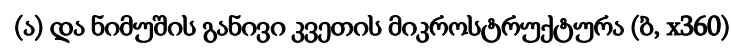

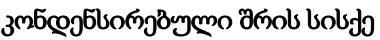

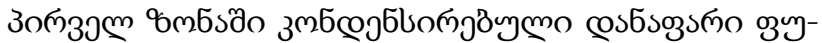

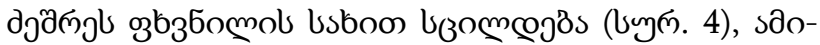

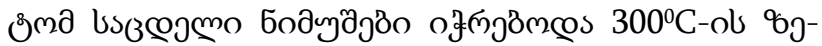

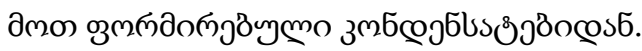

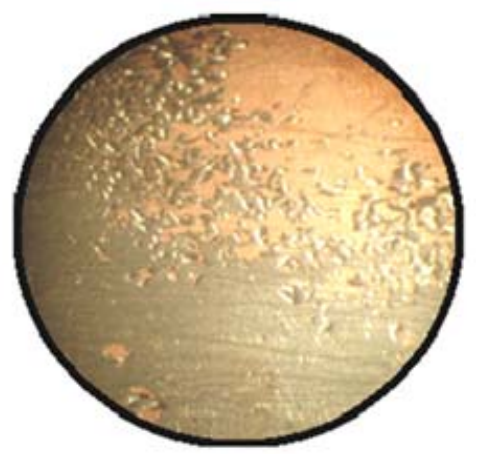

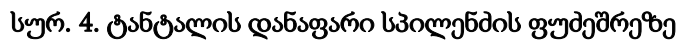

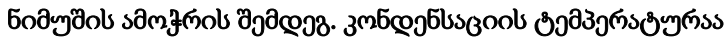
$250-300^{\circ} \mathrm{C}$

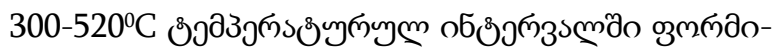

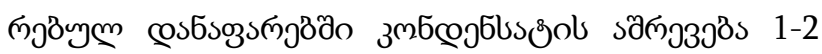

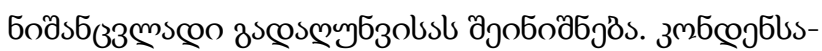

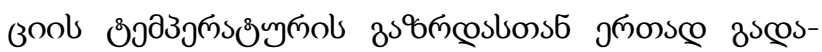

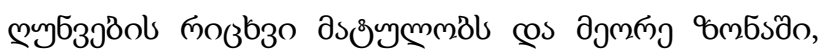

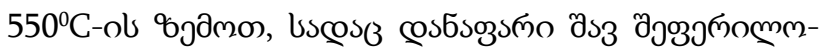

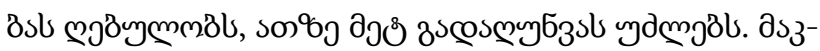

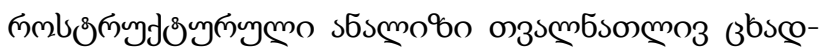

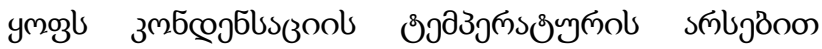

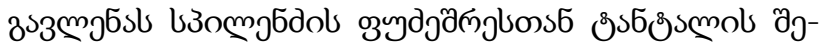

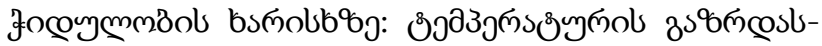

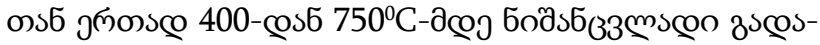

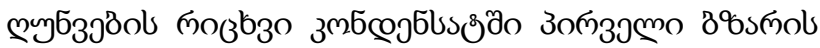

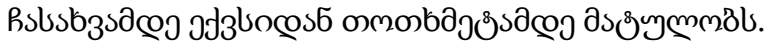

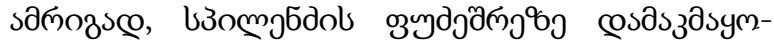

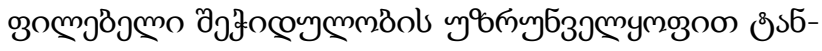

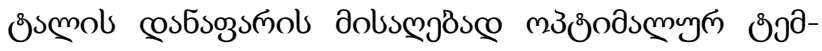

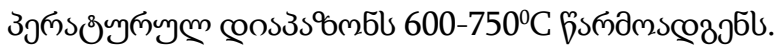




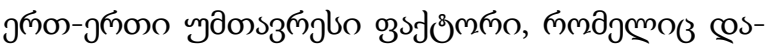

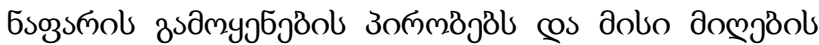

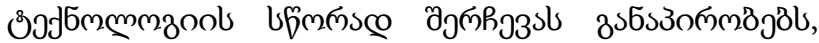

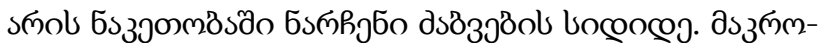

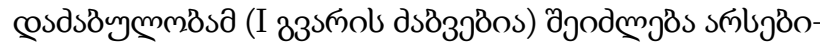

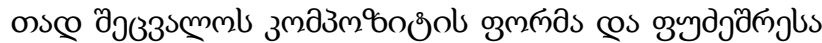

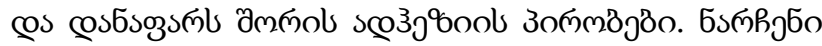

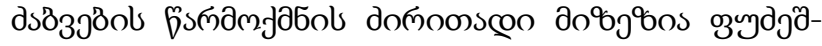
mols ces costsogs

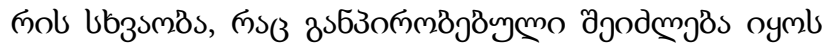

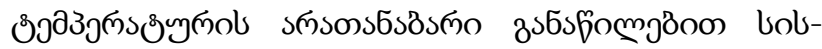

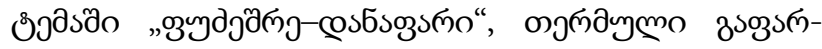

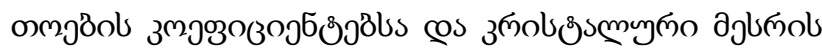

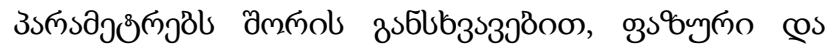

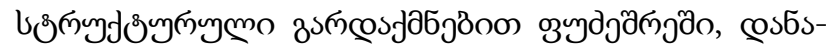

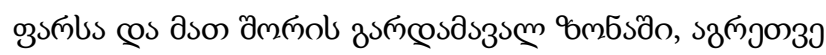

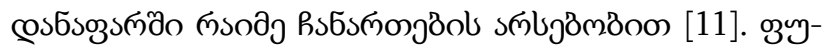

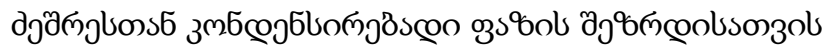

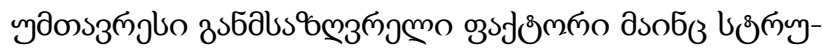

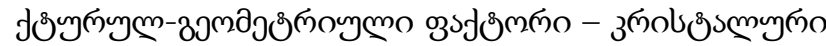

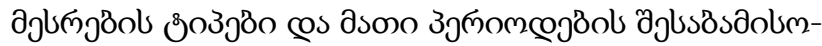
Bob bsmolbos $\left\{\Delta=\left(a_{1}-a_{2}\right) / a_{1}\right.$, bscoss $a_{1}$ smol oygdjgnols

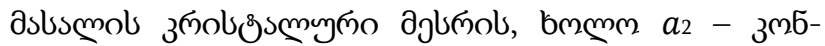

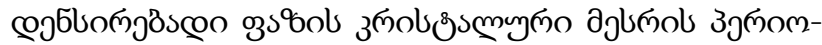
@o) $[12,17]$.

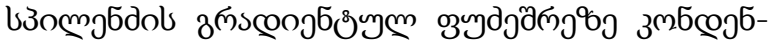

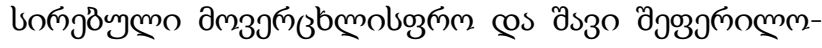

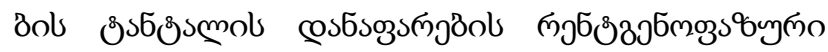

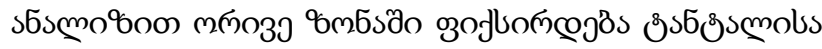

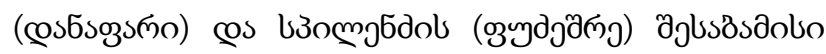

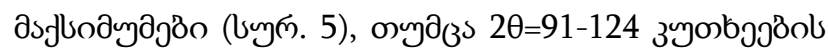

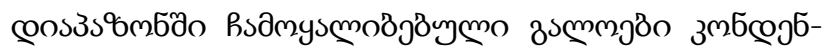

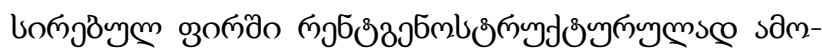

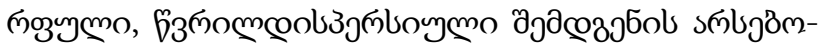

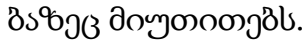

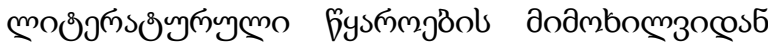

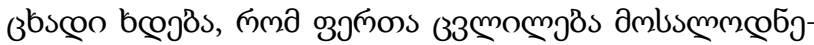

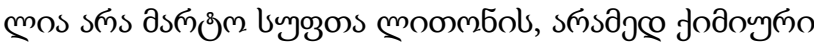

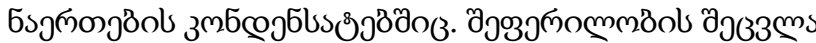

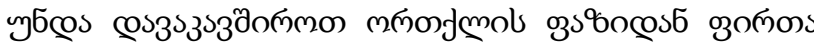

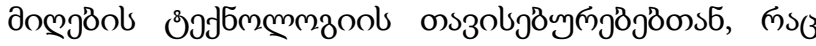

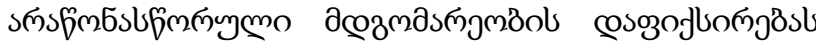

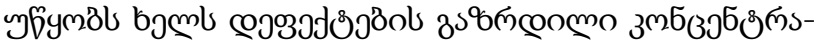

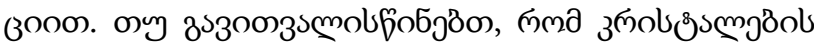

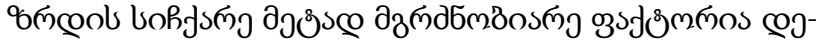

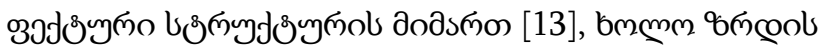

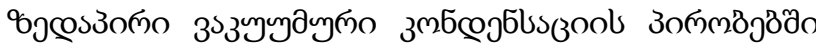

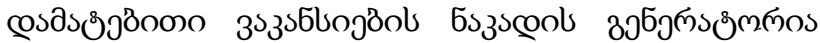

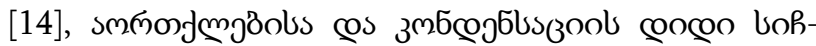

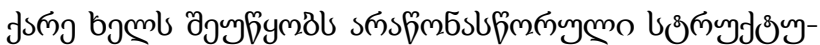

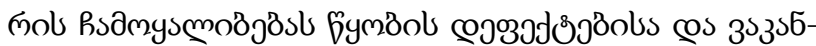

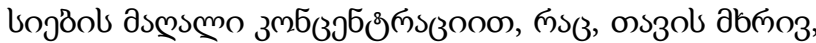

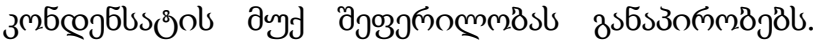

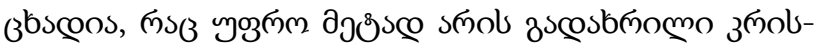

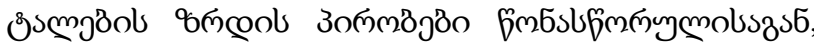

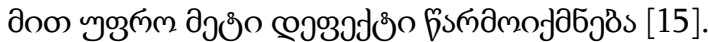

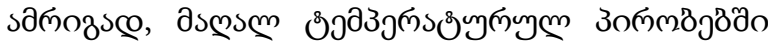

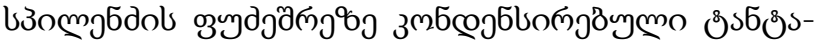

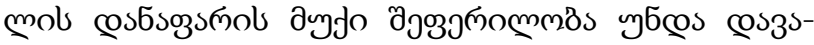

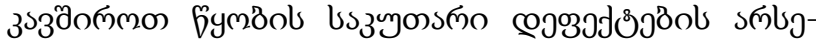
вmäslsoss6.

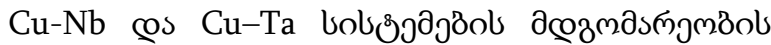

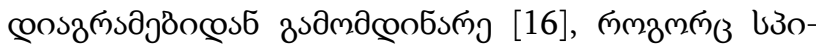

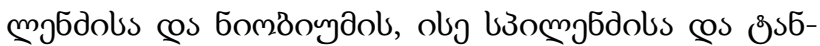

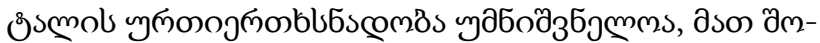

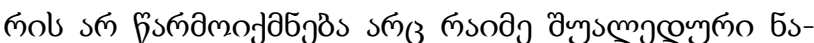




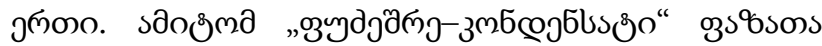

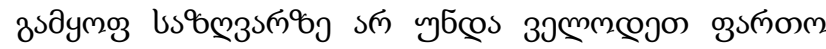

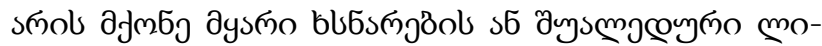

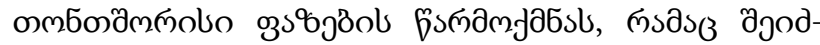

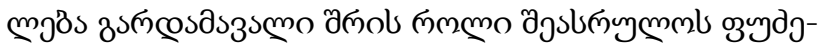

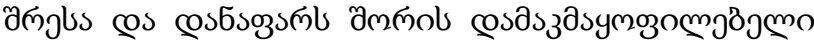

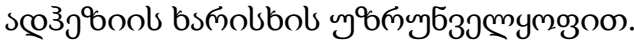
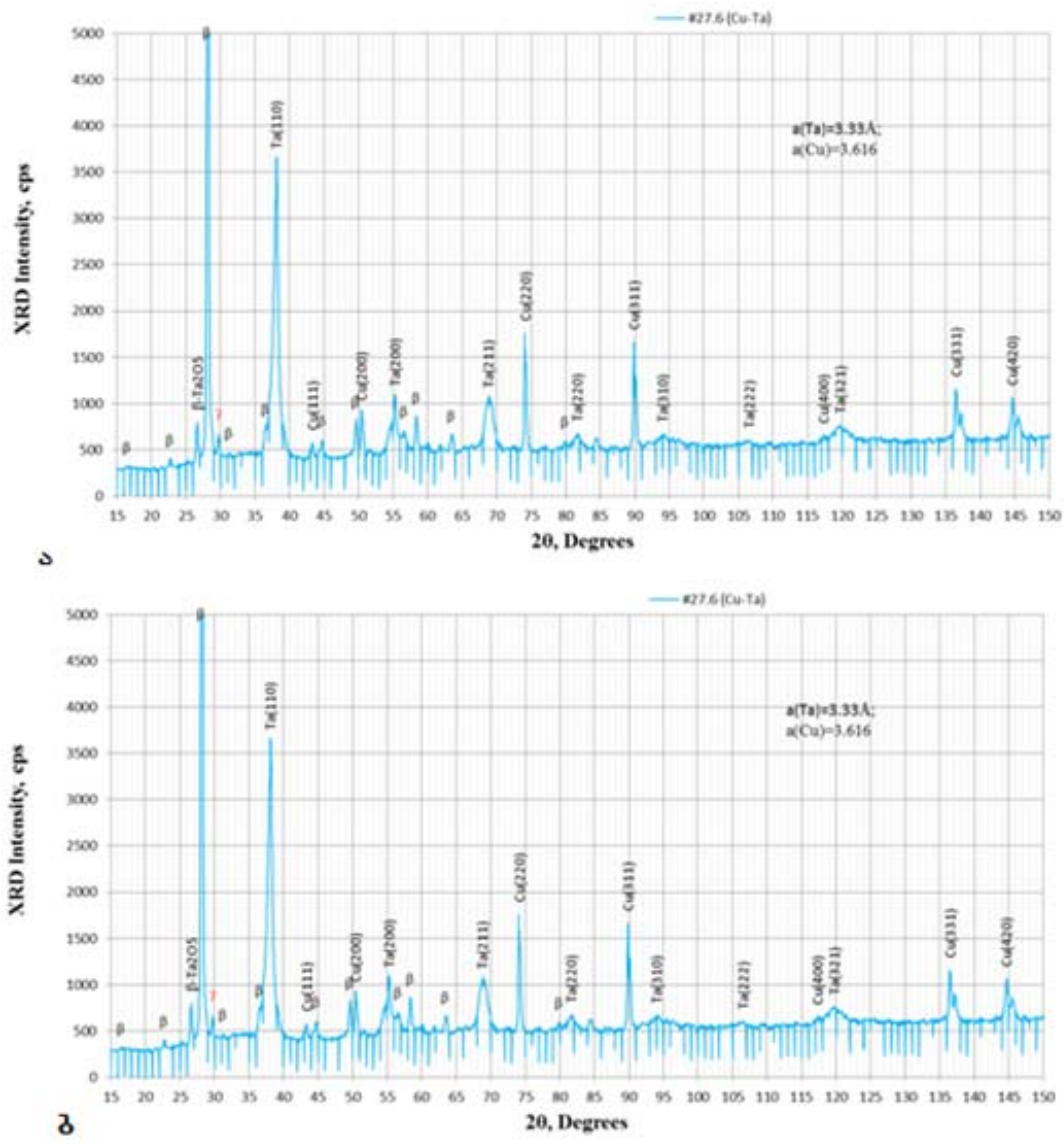

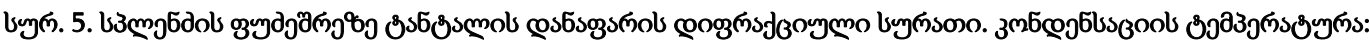
$s-<370^{\circ} \mathrm{C}$ (дмзјэп)

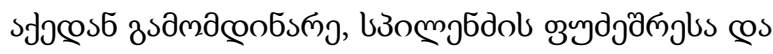

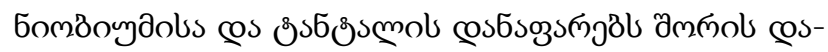

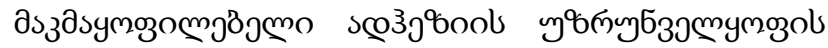
๓зs

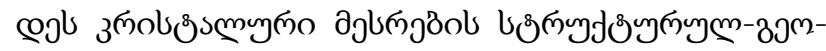

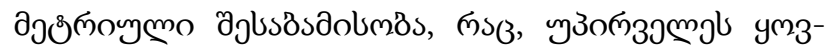

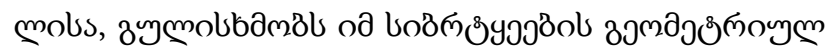

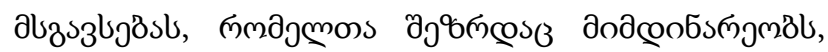

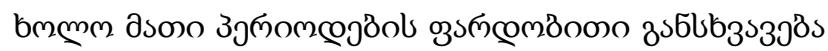

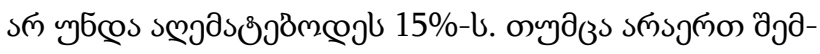

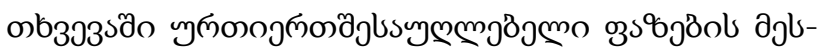

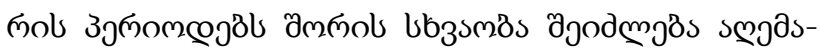

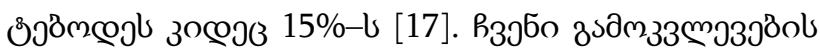

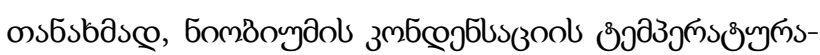
ز

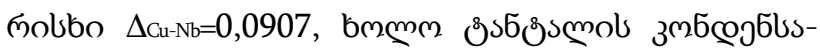

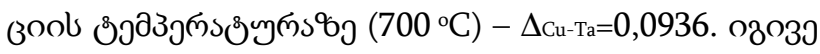

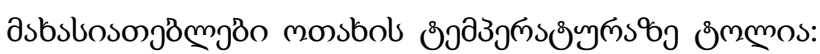




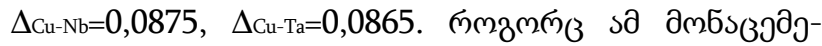

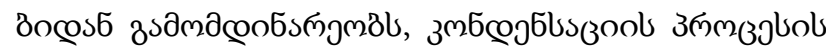

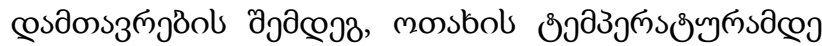

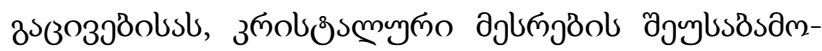

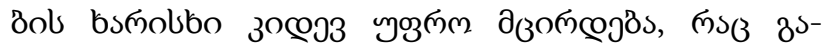

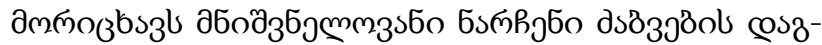

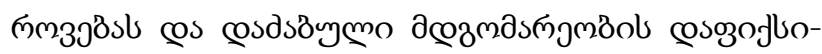

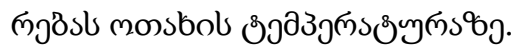

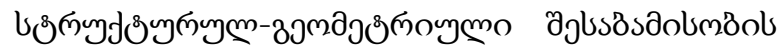

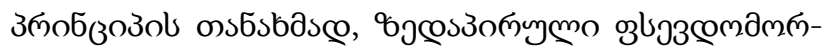

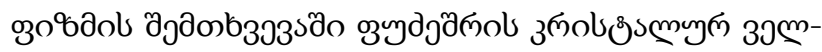

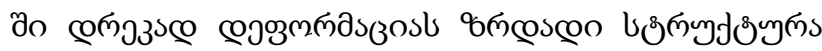

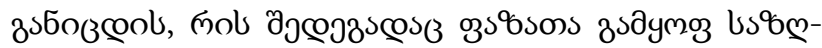

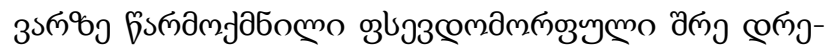

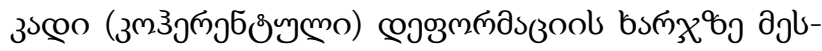

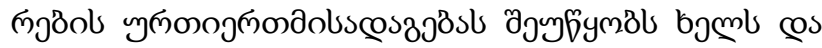

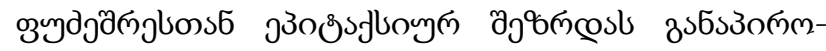

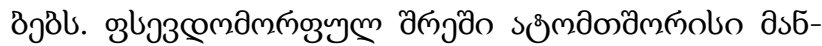

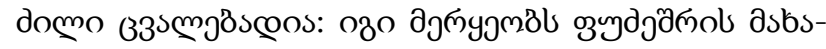

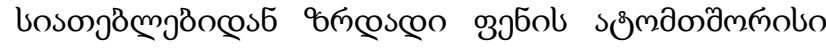

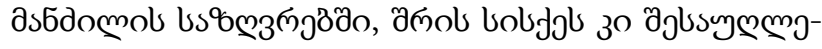

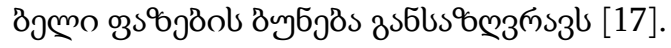

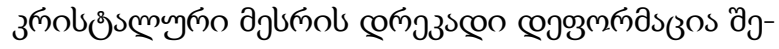

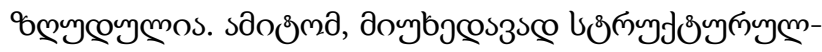

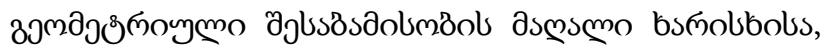

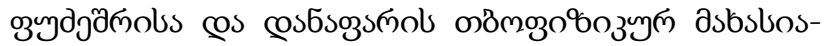

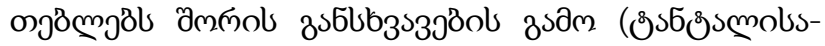

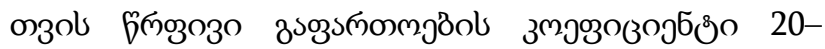

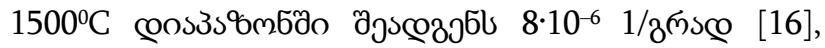

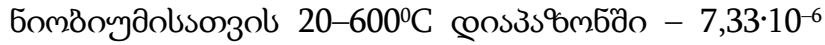

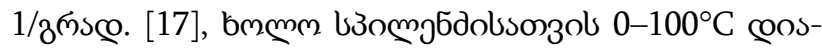

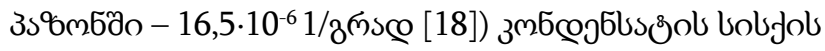

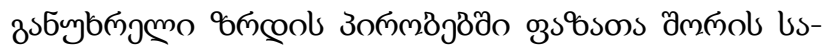

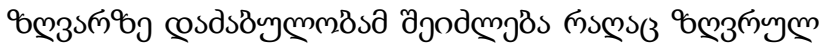

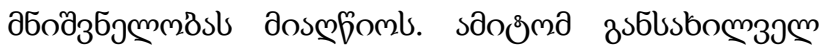

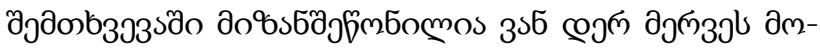

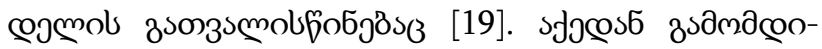

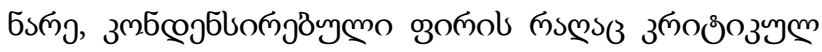

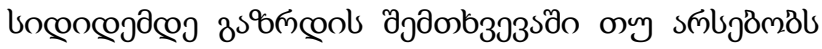

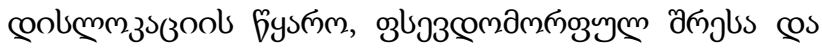

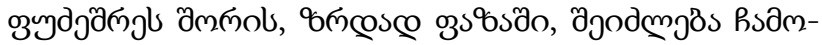

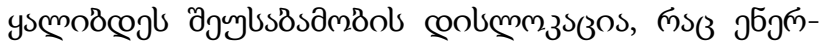

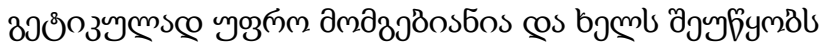

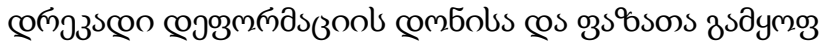

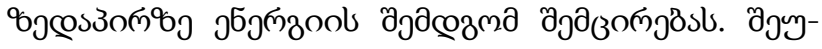

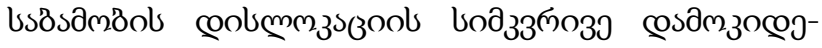

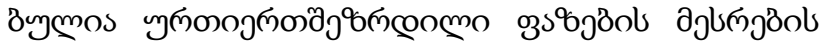

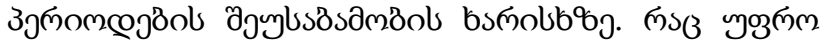

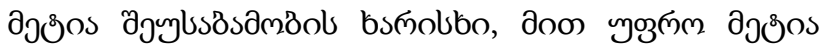

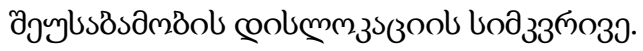

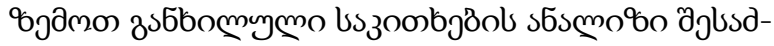

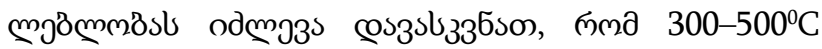
ం6తీ9ణ3క

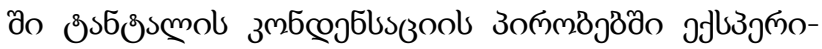

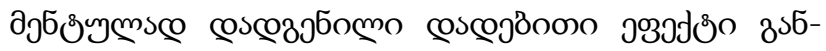

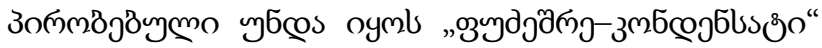

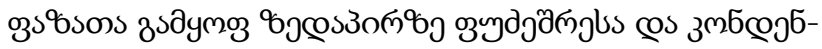

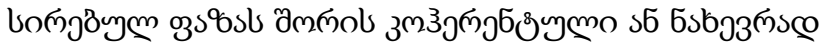

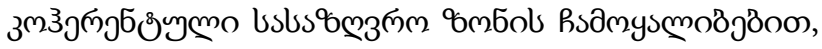

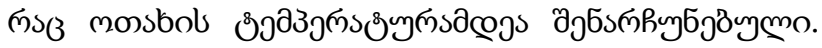

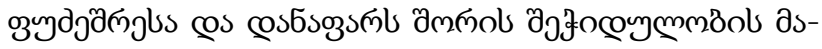

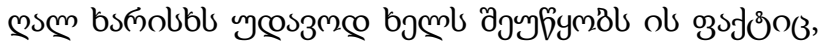

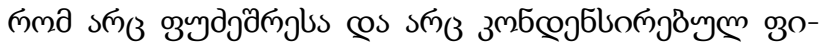

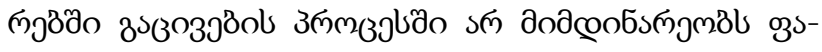

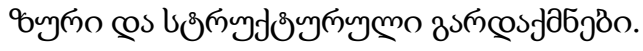




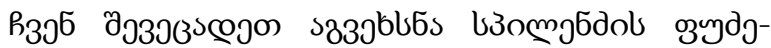

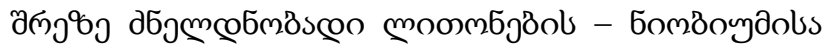

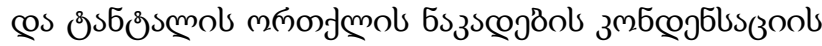

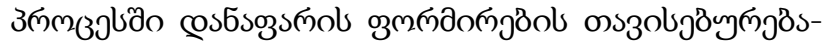

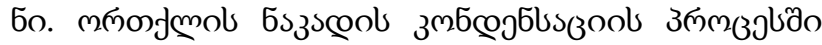

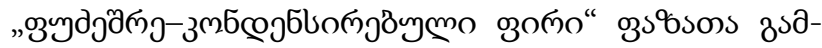

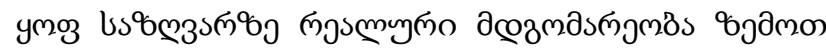

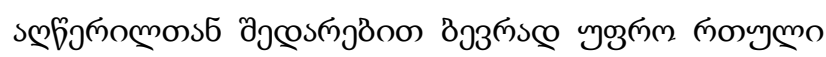

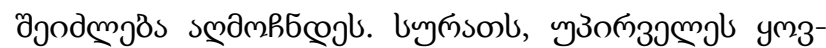

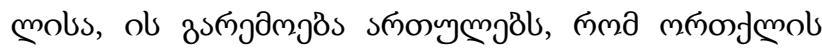

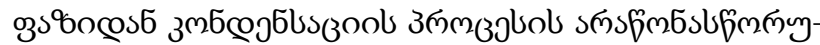

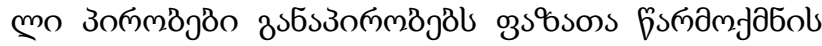

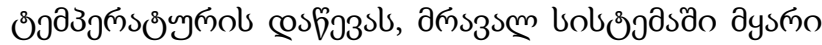

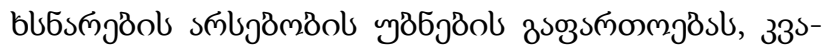

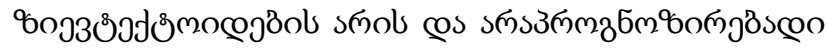

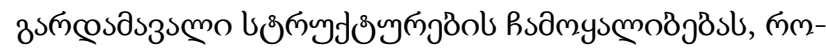

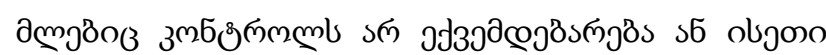

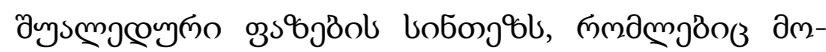

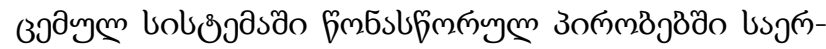

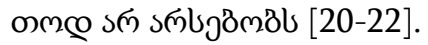

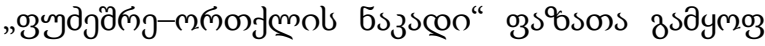

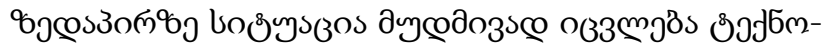

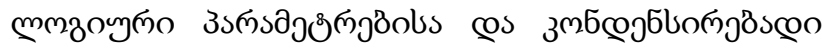

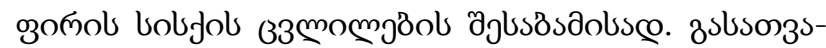

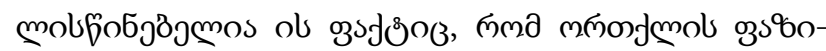

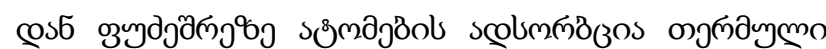

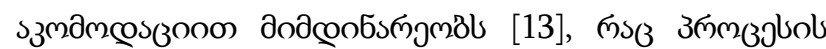

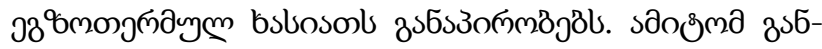

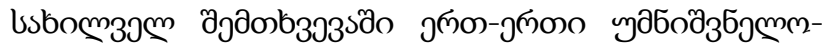

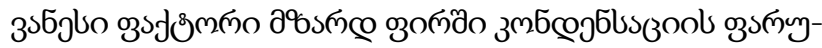

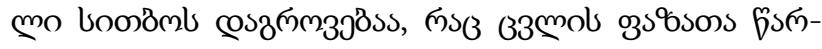

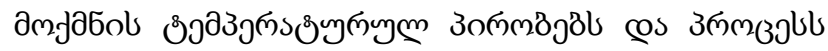

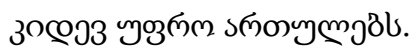

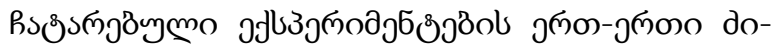

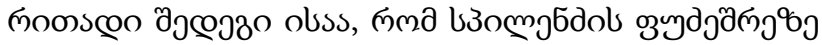

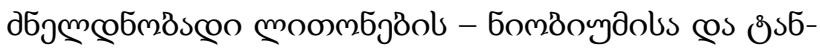

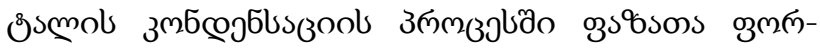

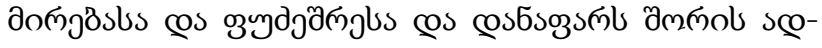

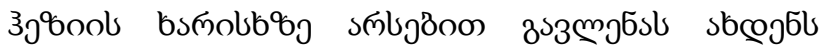

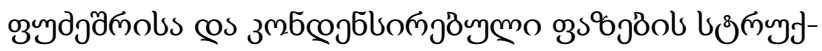

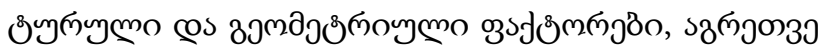

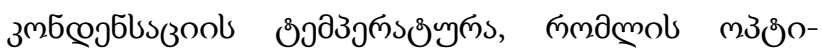

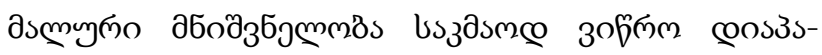

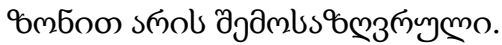

\section{cost $336 s$}

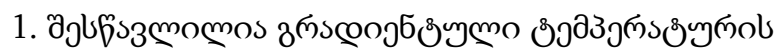

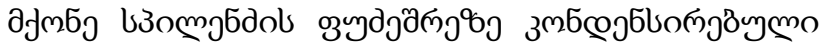

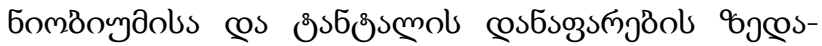

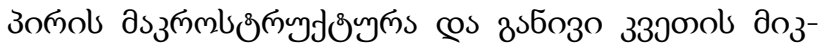

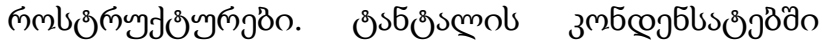

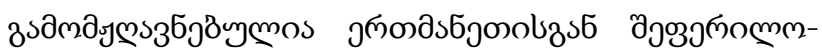

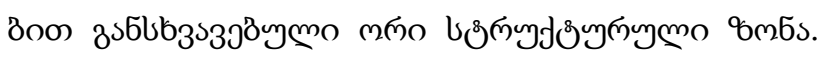

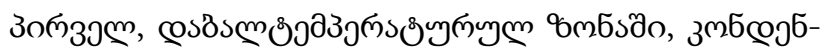

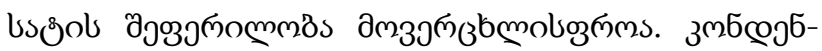

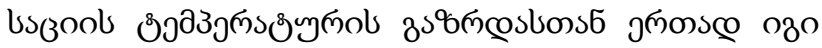

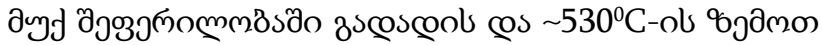

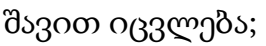

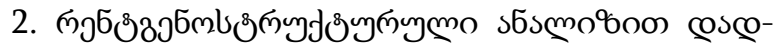

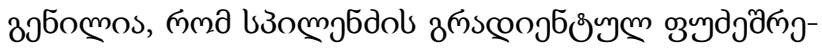

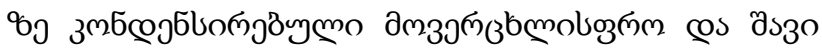

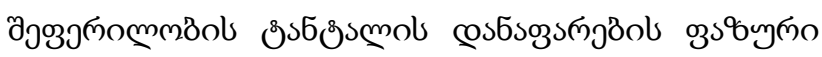

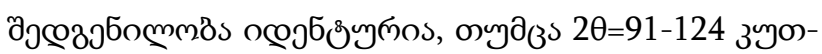

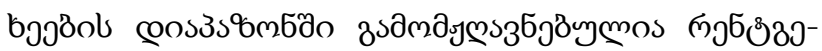

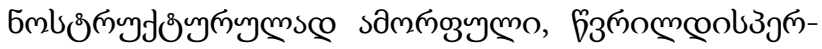

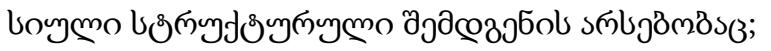




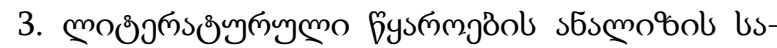

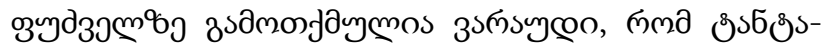

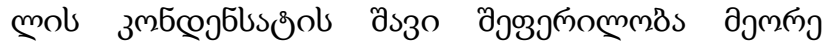

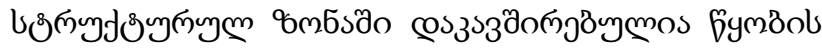

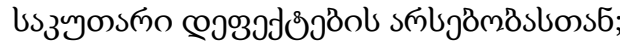

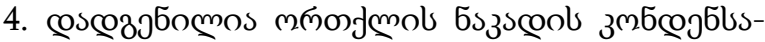

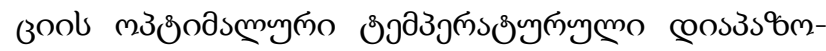

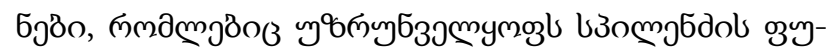

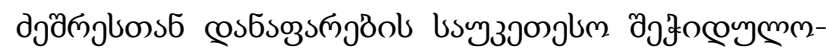

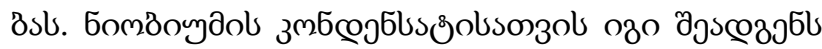
300-500

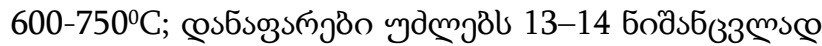

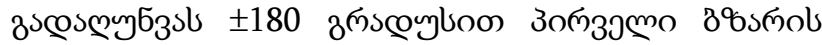

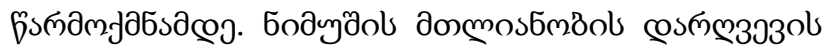

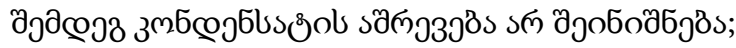

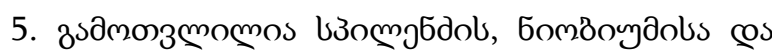

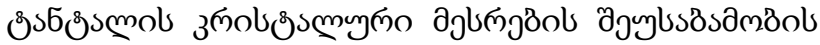

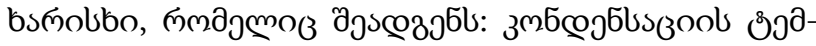

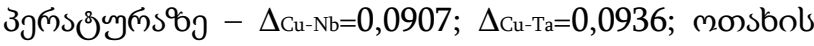

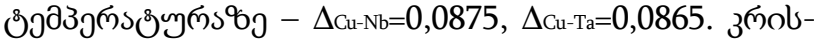

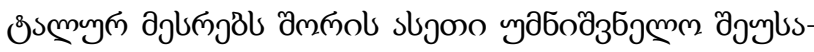

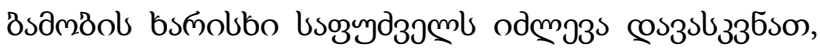

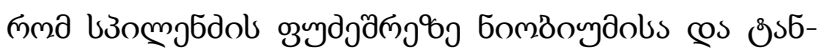

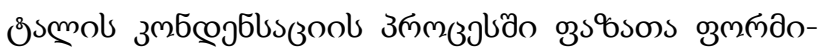

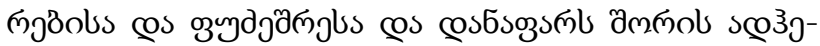

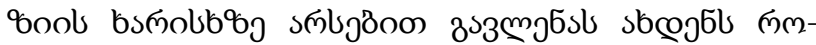

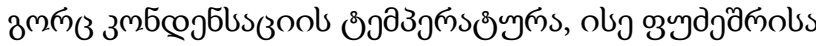

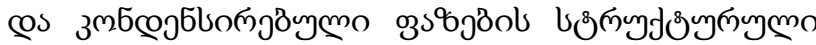

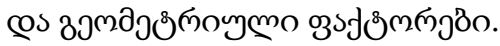

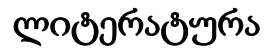

1. Mattox D. M. Handbook of Physical Vapor Deposition (PVD) processing. Noyes Publications. 1998, 945 p.

2. Bunshah R. F. Handbook of deposition technologies for films and coatings. Noyes Publications. 1994, 888 p.

3. Arshi N., Lu J., Chan Gyu Lee, Jae Hong Yoon, Bon Heun Koo, Ahmed F. Thickness effect on properties of titanium film deposited by d.c.magnetron sputtering and electron beam evaporation techniques. Bull. Mater. Sci., Vol. 36. No. 5. 2013, 807-812 pp.

4. The Copper advantage. A guide to working with Copper and Copper alloys. URL: www.antimicrobialcopper.com

5. Michel J. Introduction to Copper and Copper alloys. Copper Development Association Inc. 2013.

6. Klaver T.P.C., Thijsse B.J. Molecular dynamics simulations of $\mathrm{Cu} / \mathrm{Ta}$ and $\mathrm{Ta} / \mathrm{Cu}$ thin film growth. Journal of computer-aided materials design. 10: 2003, 61-74 pp.

7. Maslov A.A., Ostvald R.V., Shagalov V.V., Maslova E.S., Gorenyuk Yu. S. Chemical technology of Niobium and Tantalum. Isdatelstvo Tomskogo politekhnicheskogo universiteta. 2010, 5-17 pp. (in Russian).

8. "Tantalum coatings. UTL: https://npozp.ru/razrab/cvdpokr/28-ta (in Russian)

9. URL: http://metal-archive.ru/osnovy-metallurgii/1638-niobiy-i-tantal.html (in Russian).

10. Okrosashvili M.N., Razmadze G.L., Lomaia T.P., Loladze T.O., Peikrishvili A.B. Technology of obtaining Ni, Nb and Ta coatings on Aluminum substrate. Transactions of GTU. \#3 (497). Tbilisi. 2015, 161-175 pp. (in Russian).

11. Barvinok V.A. Control of the stress state and properties of Plasma coatings. M.: "Mashinostroenie". 1990, 384 p. (in Russian).

12. Palatnik L.S., Sorokin V.K. Materials science and microelectronics. M.: "Energiya". 1978, 277 p. (in Russian).

13. Strickland-Constable R.F. Kinetic and mechanism of chystallization. Leningrad. "Nedra". 1971, 310 p. (in Russian). 
14. Kosevich V. M., Karpovskyi F.V., Kosmachev S.M., Klimenko V.N. Growth surface as a generator of vacancies under vacuum condensation. Rost Kristallov Proceedings. №19. 1991, 22-39 pp. (in Russian).

15. Ruykorararn R., Sites J.R. Crystallization of Zirconia films by thermal annealing. J. Sci and Technol. A4. №3, Pt.1. 1986, 568-571 pp.

16. Barabash O.M., Koval Yu.N. Structure and properties of metals and alloys. Crystal structure of metals and alloys. Handbook. Kiev. "Naukova Dumka”. 1996, 598 p. (in Russian).

17. Maltsev M.V. Metallography of industrial non-ferrous metals and alloys. M.: "Metallurgiya”. 1970, 368 p. (in Russian).

18. Van der Merwe J. H. Monocrystalline films. M.: “Mir”. 1966, 172 p. (in Russian).

19. Okrosashvili M., Kutelia E., Makharadze T., Topuria M., Peikrishvili A. Phase formation at the condensation of a vapor stream and associated diffusion reaction. Surface engineering. Proceedings of the $5^{\text {th }}$ international surface engineering congress. Ohio. 2006, 102-105 pp.

20. Okrosashvili M.N., Kutelia E.R., Kopaleishvili V.P. Study of phase formation processes in condensates of the Ti-Cu system obtained from the vapor state. Fizika metallov i metallovedenie. Vol. 80. \#6. Nauka. 1995, 60-66 pp. (in Russian).

21. Palatnik L.S., Sorokin V.K. Fundamentals of semiconductor film materials science. M.: "Energiya”. 1973,294 p. (in Russian). 


\title{
UDC 669:187.526.001.5 \\ SCOPUS CODE 2501
}

DOI: https://doi.org/10.36073/1512-0996-2019-3-98-110

\section{Technology for obtaining of Niobium and Tantalum coatings on the copper Substrate}

\author{
Khatia Ananiashvili Department of Metallurgy, Metals Science and Metal Processing, Georgian Technical \\ University, 69 M. Kostava str, 0160 Tbilisi, Georgia \\ E-mail: kh.ananiashvili@gtu.ge \\ Mikheil Okrosashvili Department of Metallurgy, Metals Science and Metal Processing, Georgian Technical \\ University, 69 M. Kostava str, 0160 Tbilisi, Georgia \\ E-mail: m.okrosashvili@gtu.ge \\ Tamar Loladze \\ Department of Metallurgy, Metals Science and Metal Processing, Georgian Technical \\ University, 69 M. Kostava str, 0160 Tbilisi, Georgia \\ E-mail: t.loladze@gtu.ge
}

\author{
Reviewers: \\ D. Nozadze, Professor, Faculty of Chemical Technology and Metallurgy, GTU \\ E-mail: d.nozadze@gtu.ge \\ V. Gordeladze, Associate Professor, Faculty of Chemical Technology and Metallurgy, GTU \\ E-mail: v.gordeladze@gtu.ge
}

The research was supported by Shota Rustaveli National Science Foundation of Georgia (SRNSFG) [PHDF-18-736, Development of the Technology for Obtaining of Functional Coatings on the Special Substrate]

\begin{abstract}
The work is aimed at the development of technology for obtaining of coatings of refractory metals Niobium and Tantalum on a Copper substrate, by evaporating the starting material with an electron beam and further condensation of a vapor stream. The macrostructure and the microstructure of the cross section, the phase composition of the coatings, obtained on the gradient substrate, and the degree of adhesion of the condensate to the substrate have been studied. The optimum temperature ranges of the substrate, which provide the best adhesion of coatings to a Copper substrate, are determined. For Niobium condensate it is $300-500^{\circ} \mathrm{C}$, and for Tantalum condensate it is $600-750^{\circ} \mathrm{C}$. The degree of mismatch between the crystal lattices of Copper, Niobium and Tantalum was calculated. Respectively it was concluded that the condensation temperature, structural and geometric factors of the substrate and condensed phases sufficiently affect the phase composition and degree of adhesion of the condensate to the substrate during the condensation of Niobium and Tantalum on the Copper substrate.
\end{abstract}

Key words: Coating; Copper substrate; electron beam; evaporation and condensation. 


\title{
UDC 669:187.526.001.5 \\ SCOPUS CODE 2501
}

DOI: https://doi.org/10.36073/1512-0996-2019-3-98-110

\section{Технология получения ниобиевых и танталовых покрытий на медной подложке}

\author{
Хатиа Ананиашвили Департамент металлургии, материаловедения и обработки металлов, Грузинский \\ технический университет, Грузия, 0160, Тбилиси, ул. М. Костава, 69 \\ E-mail: kh.ananiashvili@gtu.ge \\ Михаил Окросашвили Департамент металлургии, материаловедения и обработки металлов, Грузинский \\ технический университет, Грузия, 0160, Тбилиси, ул. М. Костава, 69 \\ E-mail: m.okrosashvili@gtu.ge \\ Тамар Лоладзе Департамент металлургии, материаловедения и обработки металлов, Грузинский \\ технический университет, Грузия, 0160, Тбилиси, ул. М. Костава, 69 \\ E-mail: t.loladze@gtu.ge
}

\section{Рецензенты:}

Д. Нозадзе, профессор факультета химической технологии и металлургии ГТУ

E-mail: d.nozadze@gtu.ge

В. Горделадзе, ассоциированный профессор факультета химической технологии и металлургии ГТУ

E-mail: v.gordeladze@gtu.ge

„Исследование осуществилось при финансовой поддержке Национального научного фонда Грузии им. Шота Руставели (SRNSFG) [PHDF-18-736, Разработка технологии получения функциональных покрытий на подложке специального назначения]“.

Аннотация. Работа посвящена разработке технологии получения покрытий тугоплавких металлов ниобия и тантала на медной подложке путем испарения исходного материала электронным лучом и последующей конденсацией потока пара. Изучены макроструктура и микроструктура поперечного сечения, фазовый состав покрытий, полученных на градиентной подложке и степень адгезии конденсата с подложкой. Определены оптимальные температурные диапазоны подложки, которые обеспечивают лучшую адгезию покрытий с медной подложкой. Для конденсата ниобия это $300-500^{\circ} \mathrm{C}$, а для конденсата тантала - 600-750 ${ }^{\circ} \mathrm{C}$. Вычислена степень несоответствия кристаллических решеток меди, ниобия и тантала, на основе чего сделан вывод, что в процессе конденсации ниобия и тантала на медной подложке на фазовый состав, а также степень адгезии конденсата с подложкой существенно влияет как температура конденсации, так и структурные и геометрические факторы подложки и конденсированных фаз.

Ключевые слова: испарение и конденсация; медная подложка; покрытие; электронный луч.

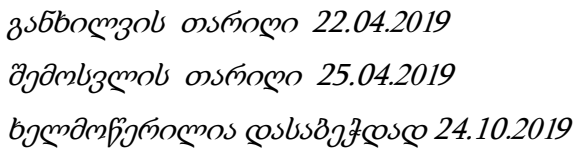


UDC 621.397.2

SCOPUS CODE 2610

DOI: https://doi.org/10.36073/1512-0996-2019-3-111-120

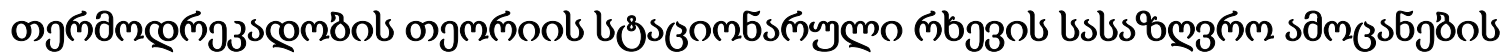

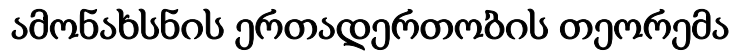

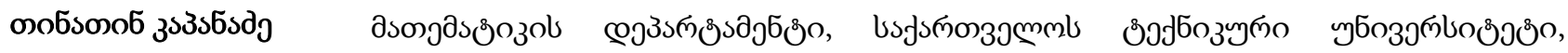

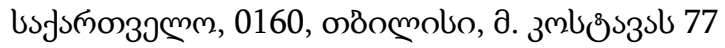

E-mail: tinatin.kapanaZe@gmail.com

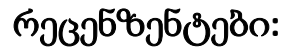

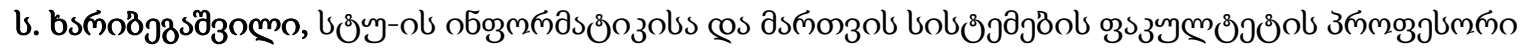

E-mail: kharibegashvili@yahoo.com

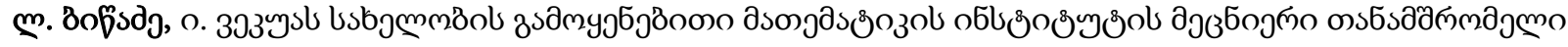

E-mail: lamarabits@yahoo.com

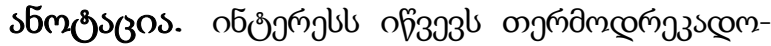

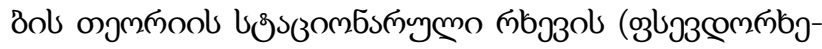

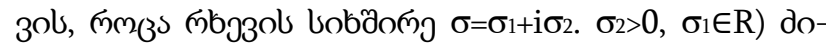

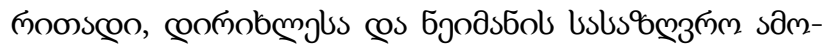

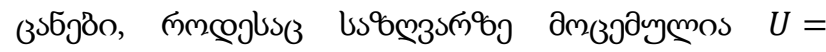

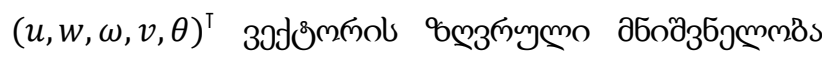

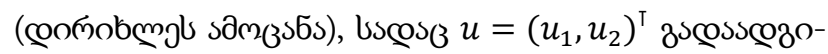

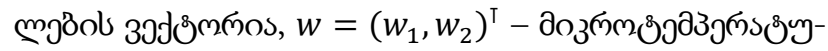

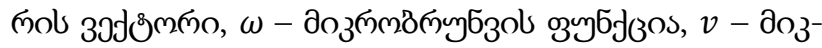

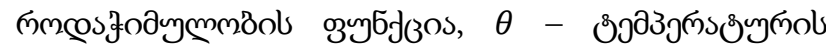

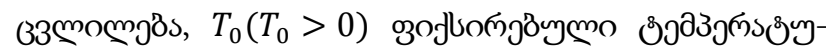

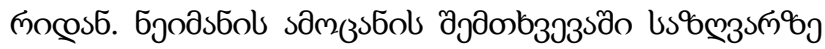

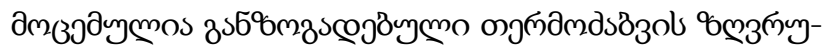

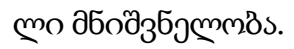

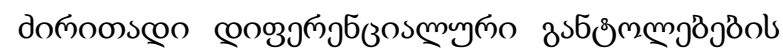

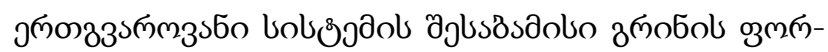

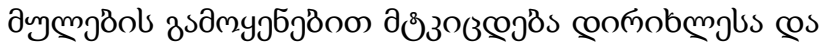

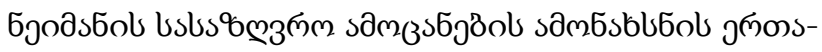

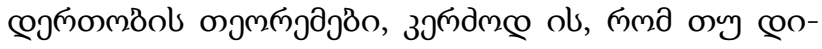

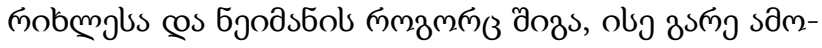

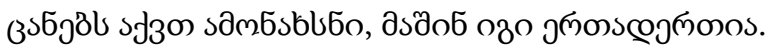

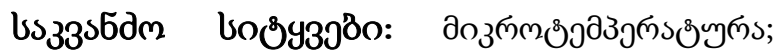

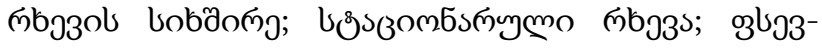

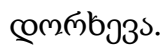

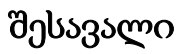

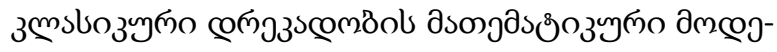
mo sল оంз

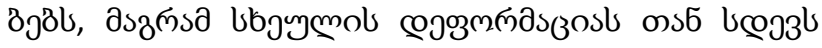

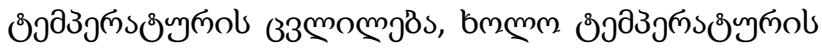

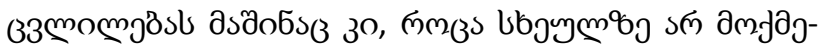




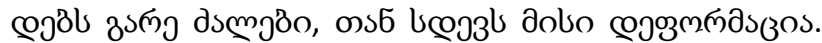

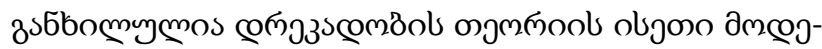

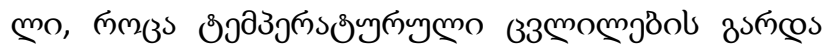

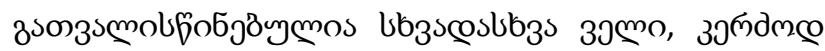

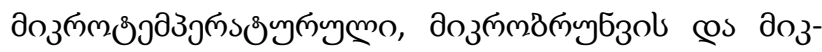

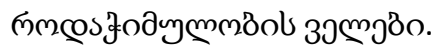

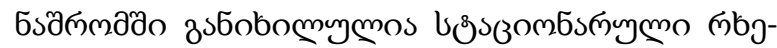

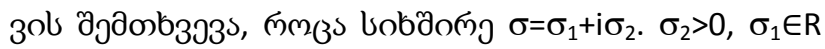

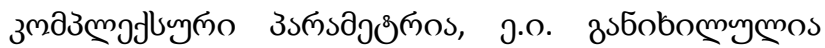

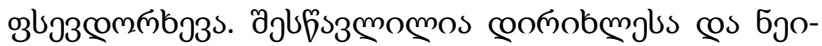

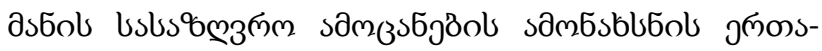

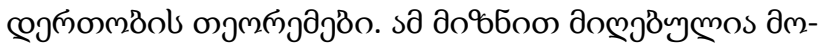

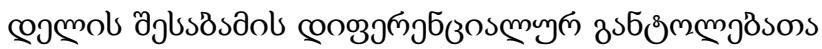

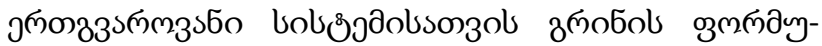

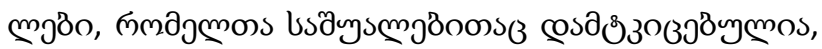

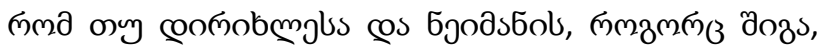

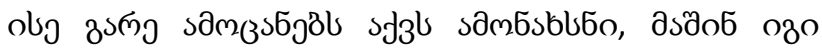

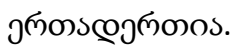

\section{donoonscon 5sfomo}

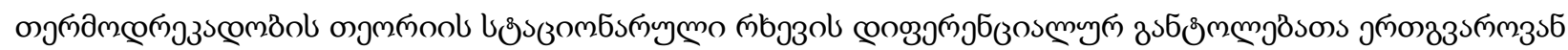

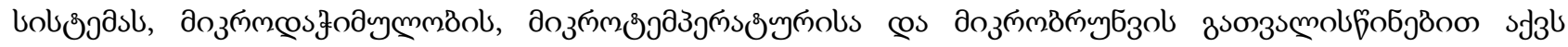

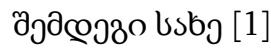

$$
\begin{aligned}
& {\left[(\mu+\varkappa) \Delta+\rho \sigma^{2}\right] u+(\lambda+\mu) \text { graddivu }-\varkappa \operatorname{rot} \omega+\mu_{0} \operatorname{grad} v-\beta_{0} \operatorname{grad} \theta=0,} \\
& \left(x_{6}+x_{0}\right) w+\left(x_{4}+x_{5}\right) \text { graddivw }+i \sigma \mu_{1} \text { rot } \omega+ \\
& +i \sigma \mu_{2} \operatorname{grad} v-x_{3} \operatorname{grad} \theta=0 \\
& (\gamma \Delta+\delta) \omega+2 \varkappa \omega+x \text { rotu }-\mu_{1} \text { rotw }=0 \\
& \left(\alpha_{0} \Delta-\eta_{0}\right) v-\mu_{0} \operatorname{divu}-\mu_{2} \operatorname{div} w+\beta_{1} \theta=0, \\
& \left(\varkappa_{7} \Delta+i \sigma C\right) \theta+i \beta_{0} T_{0} \sigma d i v u+\varkappa_{1} \operatorname{div} w+i \beta_{1} T_{0} \sigma v=0 \text {, }
\end{aligned}
$$

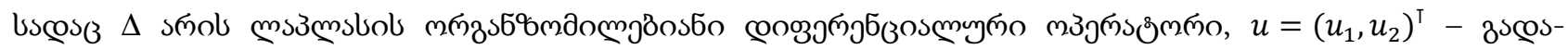

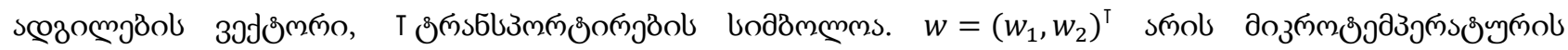

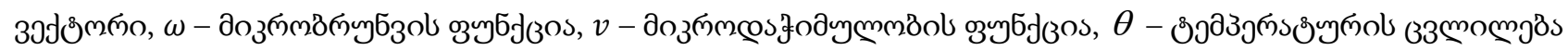

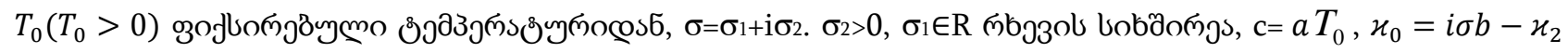
$\sigma=I_{1} \sigma^{2}-2 \varkappa ; \eta_{0}^{2}=I_{1} \sigma^{2}-\eta, \quad a, b, I, I_{1}, \gamma, \lambda, \mu, \chi, \eta, \beta_{0}, \beta_{1}, \mu_{0}, \mu_{1}, \mu_{2}, a_{0}, \varkappa_{j}, j=1,2, \ldots, 7$, бsдезомо дуюдозу

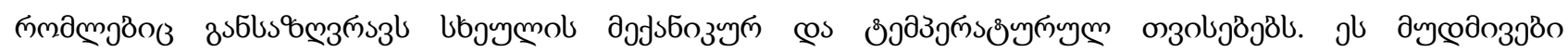

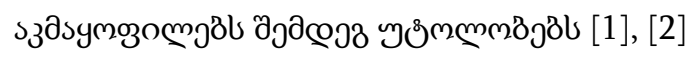

$$
\begin{gathered}
a_{0}>0, \mu>0, \eta>0, \gamma>0, \varkappa>0,2 \lambda+2 \mu+\varkappa-2 \mu_{0}^{2}>0, a_{0} \gamma- \\
-b_{0}^{2}>0, \varkappa_{7}>0,\left(\varkappa_{1}+\varkappa_{3} T_{0}\right)^{2} \leq 4 T_{0} \varkappa_{2} \varkappa_{7},
\end{gathered}
$$




$$
\varkappa_{6} \pm \varkappa_{5} \geq 0,2 \varkappa_{4}+\varkappa_{5}+\varkappa_{6}>0, \mu+\lambda>0 .
$$

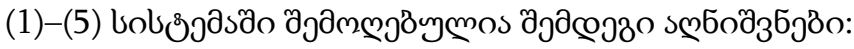

$$
\begin{aligned}
& \operatorname{rot}:=\left(-\frac{\partial}{\partial x_{2}}, \frac{\partial}{\partial x_{1}}\right)^{\top}, \operatorname{rot} \omega:=\left(-\frac{\partial \omega}{\partial x_{2}}, \frac{\partial \omega}{\partial x_{1}}\right)^{\top} \\
& \text { rotu }:=\frac{\partial u_{2}}{\partial x_{1}}-\frac{\partial u_{1}}{\partial x_{2}}, \operatorname{rotw}:=\frac{\partial w_{2}}{\partial x_{1}}-\frac{\partial w_{1}}{\partial x_{2}},
\end{aligned}
$$

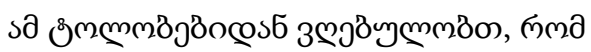

$$
\begin{aligned}
& \text { rotrot } \omega=\Delta \omega, \text { rotrotu }=\Delta u-\text { graddivu }, \\
& \text { rotgrad }=0, \text { divrot }=0 .
\end{aligned}
$$

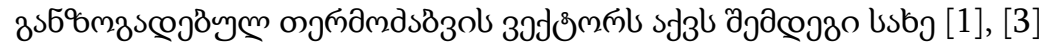

$$
P(\partial, n) U=\left(T^{(1)}(\partial, n) U, T^{(2)}(\partial, n) U, T^{(3)}(\partial, n) U, T^{(4)}(\partial, n) U, T^{(5)}(\partial, n) U\right)^{T},
$$

uscosos

$$
\begin{gathered}
T^{(1)}(\partial, n) U:=(2 \mu+\varkappa) \frac{\partial u}{\partial n}+\lambda n \text { divu }-(\mu \text { rotu }+\varkappa \omega) s+\left(\mu_{0} v-\beta_{0} \theta\right) n, \\
T^{(2)}(\partial, n) U:=\left(\varkappa_{5}+\varkappa_{6}\right) \frac{\partial w}{\partial n}+\varkappa_{4} n \text { divw }-\varkappa_{5} s \text { rotw }, \\
T^{(3)}(\partial, n) U:=\gamma \frac{\partial \omega}{\partial n}-\mu_{1}(s \cdot w)-b_{0}(s \cdot \operatorname{grad} v), \\
T^{(4)}(\partial, n) U:=a_{0} \frac{\partial v}{\partial n}-\mu_{2}(n \cdot w)+b_{0}(s \cdot \operatorname{grad} \omega), \\
T^{(5)}(\partial, n) U:=\mu_{7} \frac{\partial \theta}{\partial n}+\varkappa_{1}(n \cdot w) .
\end{gathered}
$$

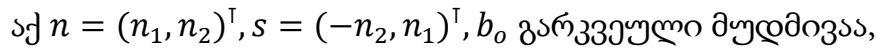

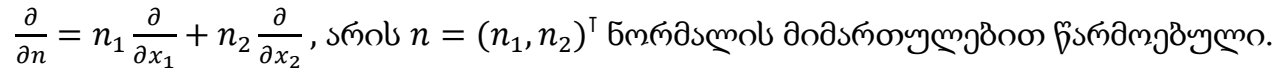

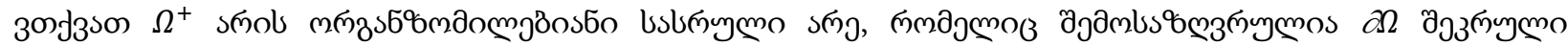
fonоo, $\Omega^{-}=R^{2} \backslash \bar{\Omega}^{+}$.

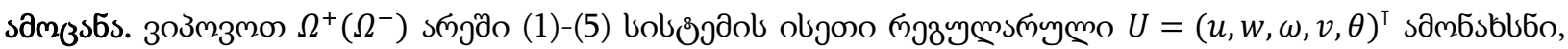

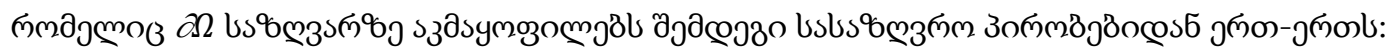

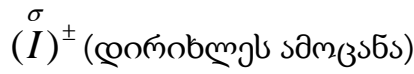

$$
\{U(z)\}^{+}=f(z),\left(\{U(z)\}^{-}=f(z)\right)
$$

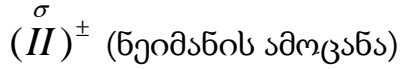

$$
\{P(\partial, n) U(z)\}^{+}=f(z),\left(\{P(\partial, n) U(z)\}^{-}=f(z)\right),
$$

$\operatorname{liscos} 3$

$$
f=\left(f^{(1)}, f^{(2)}, f_{3}, f_{4}, f_{5}\right)^{\top}, f^{(j)}=\left(f_{1}^{(j)}, f_{2}^{(j)}\right)^{\top}, j=1,2,
$$




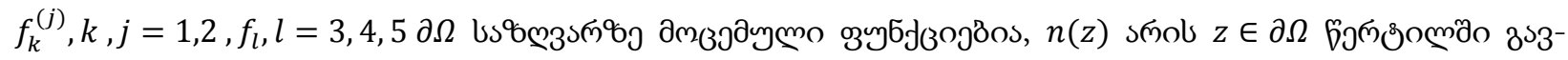

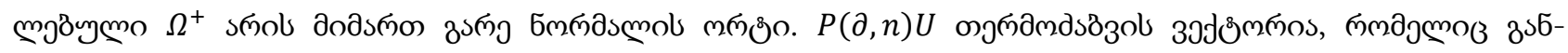

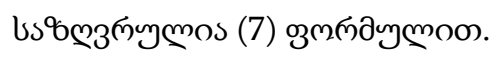

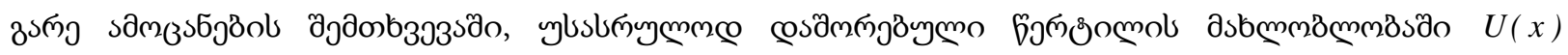

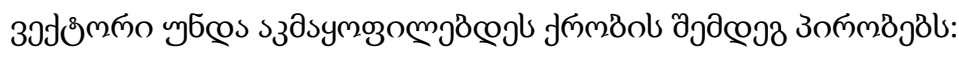

$$
U(x)=O\left(|x|^{-1}\right), \partial_{k} U(x)=o\left(|x|^{-1}\right), k=1,2 .
$$

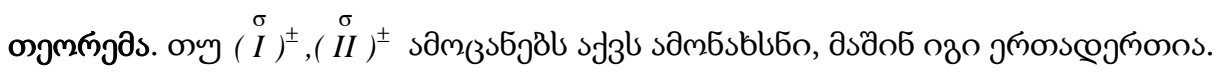

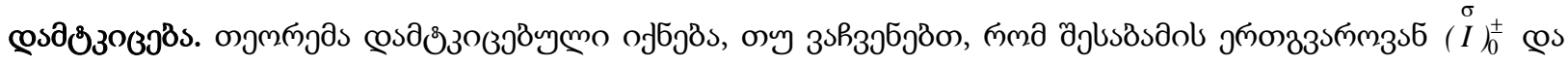
$(\stackrel{\sigma}{I I})_{0}^{ \pm} \quad(f(z)=0, z \in \partial \Omega)$ sам

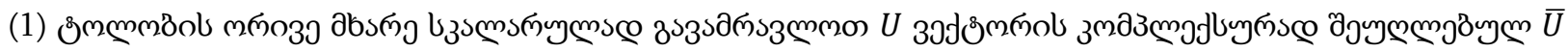

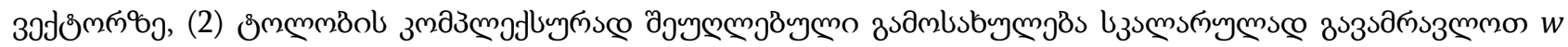

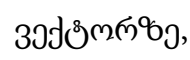

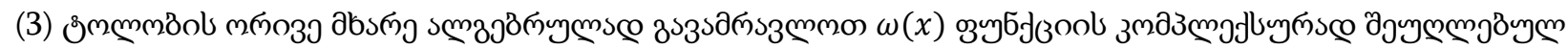

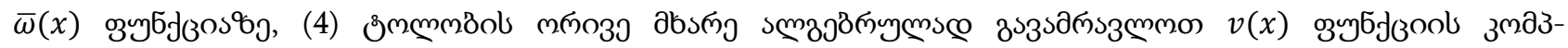

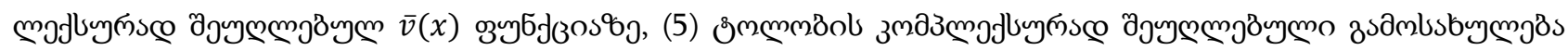

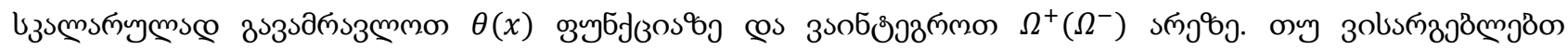

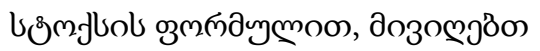

$$
\begin{gathered}
\pm \int_{\partial \Omega}\left\{\bar{u}(z) \cdot T^{(1)}(\partial, n) U(z)\right\}^{ \pm} d s-\int_{\Omega^{ \pm}}\left[E^{(1)}(\bar{u}, u)+\rho \sigma^{2}|u(x)|^{2}+\right. \\
\left.+\mu_{0} v(x) \operatorname{div} \bar{u}(x)-\beta_{0} \theta(x) \operatorname{div} \bar{u}(x)-x \omega \operatorname{rrot} \bar{u}(x)\right] d x=0 \\
\pm \int_{\partial \Omega}\left\{\bar{w}(z) \cdot T^{(2)}(\partial, n) \bar{U}(z)\right\}^{ \pm} d s-\int_{\Omega^{ \pm}}\left[E^{(2)}(\bar{w}, w)-\bar{x}_{0}|w(x)|^{2}+\right. \\
\left.+i \bar{\sigma} \mu_{1} w(x) \operatorname{rot} \bar{\omega}(x)+i \bar{\sigma} \mu_{2} w(x) \operatorname{grad} \bar{v}(x)+x_{3} w(x) \operatorname{grad} \bar{\theta}(x)\right] d x=0 \\
\pm \int_{\partial \Omega}\left\{\bar{\omega}(z) \cdot T^{(3)}(\partial, n) U(z)\right\}^{ \pm} d s-\int_{\Omega^{ \pm}}\left[\gamma|\operatorname{grad} \omega(x)|^{2}+-\delta|\omega(x)|^{2}-2 x \omega^{2}-\right. \\
\left.-x \bar{\omega}(x) \operatorname{rotu}(x)-\mu_{1} w(x) \operatorname{rot} \bar{\omega}(x)-b_{0} \operatorname{grad} v(x) \operatorname{rot} \bar{\omega}(x)\right] d x=0 \\
\pm \int_{\partial \Omega}\left\{\bar{v}(z) \cdot T^{(4)}(\partial, n) U(z)\right\}^{ \pm} d s-\int_{\Omega^{ \pm}}\left[a_{0}|\operatorname{gradv}(x)|^{2}-\eta_{0}|v(x)|^{2}+\right. \\
\left.+\mu_{0} \bar{v}(x) \operatorname{divu}(x)-\beta_{1} \bar{v}(x) \theta(x)-\mu_{2} w(x) \operatorname{grad} \bar{v}(x) \cdot \operatorname{rot} \omega(x)\right] d x=0
\end{gathered}
$$




$$
\begin{aligned}
& \pm \int_{\partial \Omega}\left\{\theta(z) \cdot T^{(5)}(\partial, n) \bar{U}(z)\right\}^{ \pm} d s-\int_{\Omega^{ \pm}}\left[x_{7}|\operatorname{grad} \theta(x)|^{2}+x_{1} \bar{w}(x) \operatorname{grad} \theta(x)+\right. \\
& \left.+i \bar{\sigma} c|\theta(x)|^{2}+i \bar{\sigma} \beta_{0} T_{0} \theta(x) \operatorname{div} \bar{u}(x)+i \bar{\sigma} \beta_{1} T_{0} \theta(x) \bar{v}(x)\right] d x=0
\end{aligned}
$$

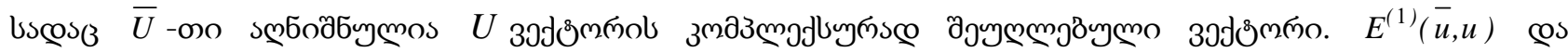

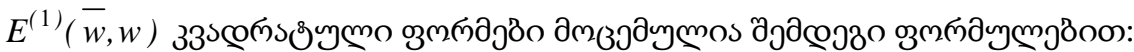

$$
\begin{aligned}
& E^{(1)}(\bar{u}, u) d x=\frac{2 \lambda+2 \mu+x}{2}|\operatorname{divu}|^{2}+\frac{2 \mu+x}{2}\left[\left|\frac{\partial u_{2}}{\partial x_{1}}+\frac{\partial u_{1}}{\partial x_{2}}\right|^{2}+\right. \\
& \left.+\left|\frac{\partial u_{1}}{\partial x_{1}}+\frac{\partial u_{2}}{\partial x_{2}}\right|^{2}\right]+\frac{x}{2} \mid \text { rotu }\left.\right|^{2} \\
& E^{(2)}(\bar{w}, w)=\frac{2 x_{4}+x_{5}+x_{6}}{2}|\operatorname{divw}|^{2}+\frac{x_{5}+x_{6}}{2}\left[\left|\frac{\partial w_{1}}{\partial x_{2}}+\frac{\partial w_{2}}{\partial x_{1}}\right|^{2}+\right. \\
& \left.+\left|\frac{\partial w_{1}}{\partial x_{1}}-\frac{\partial w_{2}}{\partial x_{2}}\right|^{2}\right]+\frac{x_{6}-x_{5}}{2}|\operatorname{rotw}|^{2}
\end{aligned}
$$

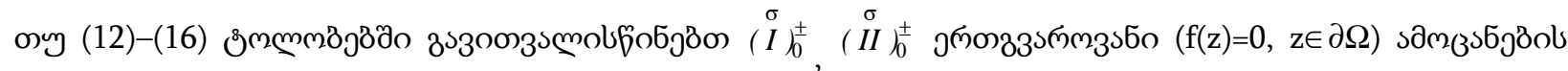

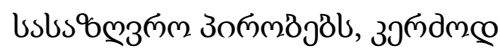

$$
\begin{aligned}
& \{\bar{U}(z)\}^{ \pm}=0,\{w(z)\}^{ \pm}=0,\{\bar{\omega}(z)\}^{ \pm}=0,\{\bar{v}(z)\}^{ \pm}=0,\{\theta(z)\}^{ \pm}=0, \\
& \left\{T^{(j)}(\partial, n) U(z)\right\}^{ \pm}=0, \quad j=1,3,4, T^{(j)}(\partial, n) \bar{U}(z)=0, j=2,5,
\end{aligned}
$$

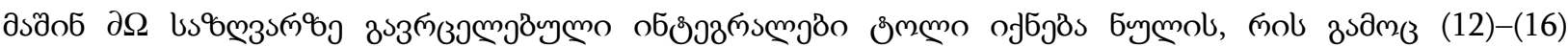

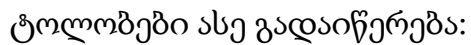

$$
\begin{gathered}
\int_{\Omega^{ \pm}}\left[E^{(1)}(\bar{u}, u)+\mu_{0} v(x) \operatorname{div} \bar{u}(x)-\beta_{0} \theta(x) \operatorname{div} \bar{u}(x)-\right. \\
\left.-\rho \sigma^{2}|u(x)|^{2}-x \omega(x) \operatorname{rot} \bar{u}(x)\right] d x=0 \\
\int_{\Omega^{ \pm}}\left[E^{(2)}(\bar{w}, w)+\overline{x_{0}}|w(x)|^{2}+i \bar{\sigma} \mu_{1} w(x) \cdot \operatorname{rot} \bar{\omega}(x)+i \bar{\sigma} \mu_{2} w(x) \cdot \operatorname{grad} \bar{v}(x)\right. \\
\left.+x_{3} w(x) \cdot \operatorname{grad} \bar{\theta}(x)\right] d x=0, \\
\int_{\Omega^{ \pm}}\left[\gamma|\operatorname{grad} \omega(x)|^{2}-\delta|\omega(x)|^{2}-x \bar{\omega}(x) \operatorname{rotu}(x)-\right. \\
\left.-\mu_{1} w(x) \cdot \operatorname{rot} \bar{\omega}(x)-b_{0} \operatorname{gradv}(x) \cdot \operatorname{rot} \bar{\omega}(x)\right] d x=0,
\end{gathered}
$$




$$
\begin{aligned}
& \int_{\Omega^{ \pm}}\left[a_{0}|\operatorname{grad} v(x)|^{2}-\eta_{0}|v(x)|^{2}+\mu_{0} \bar{v}(x) \operatorname{divu}(x)-\right. \\
& \left.-\beta_{1} \bar{v}(x) \theta(x)-\mu_{2} w(x) \cdot \operatorname{grad} \bar{v}(x)-b_{0} \operatorname{grad} \bar{v}(x) \cdot \operatorname{rot} \omega(x)\right] d x=0 \\
& \quad \int_{\Omega^{ \pm}}\left[x_{7}|\operatorname{grad} \theta(x)|^{2}+x_{1} \bar{w}(x) \cdot \operatorname{grad} \theta(x)+i \bar{\sigma} c|\theta(x)|^{2}+\right. \\
& \left.\quad+i \bar{\sigma} \beta_{0} T_{0} \theta(x) \operatorname{div} \bar{u}(x)+i \bar{\sigma} \beta_{1} T_{0} \theta(x) \bar{v}(x)\right] d x=0
\end{aligned}
$$

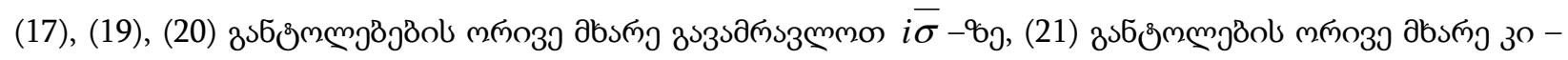

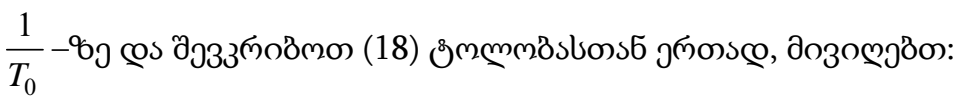

$$
\begin{aligned}
& \int_{\Omega^{ \pm}}\left[i \bar{\sigma} E^{(1)}(\bar{u}, u)+E^{(2)}(\bar{w}, w)-i \bar{\sigma} \sigma^{2} \rho|u|^{2}-x_{0}|w|^{2}-i \bar{\sigma} \delta|\omega|^{2}+\right. \\
& +i \bar{\sigma} a_{0}|\operatorname{grad} v|^{2}+\frac{x_{7}}{T_{0}}|\operatorname{grad} \theta|^{2}+i \bar{\sigma} \mu_{0}(v \operatorname{div} \bar{u}+\bar{v} \operatorname{divu})- \\
& -i \bar{\sigma} x(\omega \operatorname{rrot} \bar{u}+\bar{\omega} \operatorname{rotu})+x_{3} w \cdot \operatorname{grad} \bar{\theta}+\frac{x_{1}}{T_{0}} \bar{w} \cdot \operatorname{grad} \theta- \\
& -i \bar{\sigma} b_{0}(\operatorname{grad} \bar{v}(x) \operatorname{rot} \omega(x)+\operatorname{grad}(x) \operatorname{rot} \bar{\omega}(x) \\
& \left.-i \bar{\sigma} \eta_{0}|v|^{2}+\frac{i \bar{\sigma}}{T_{0}}|\theta|^{2}-i \bar{\sigma} \gamma|\operatorname{grad}(\omega)|^{2}(x)\right] d x=0
\end{aligned}
$$

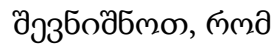

$$
i \bar{\sigma} \sigma^{2}=\left(-\sigma_{2}+i \sigma_{1}\right)|\sigma|^{2}, \overline{\varkappa_{0}}=-\left(\varkappa_{2}+\sigma_{2} b\right)-i \sigma_{1} b,
$$

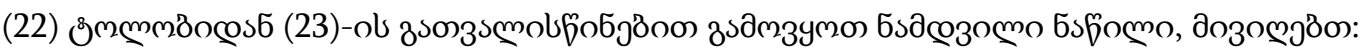

$$
\begin{aligned}
& \int_{\Omega^{ \pm}}\left[\sigma_{2} E^{(1)}(\bar{u}, u)+E^{(2)}(\bar{w}, w)-\rho \sigma_{2}|\sigma|^{2}|u|^{2}+\left(\sigma_{2} b+\varkappa_{2}\right)|w|^{2}+\right. \\
& +\sigma_{2}\left(I_{1}|\sigma|^{2}+2 \varkappa\right)|\omega|^{2}+\sigma_{2} a_{0}|\operatorname{grad} v|^{2}+\frac{x_{7}}{T_{0}}|\operatorname{grad} \theta|^{2}+ \\
& \sigma_{2}\left(I|\sigma|^{2}+\eta\right)|v|^{2}+\frac{\sigma_{2} c}{T_{0}}|\theta|^{2}+\sigma_{2} \mu_{0}(v \operatorname{div} \bar{u}+\bar{v} \operatorname{divu}) \\
& +\sigma_{2} \varkappa(\omega \operatorname{rot} \bar{u}+\bar{\omega} r o t u)+\frac{x_{1}+T_{0} \varkappa_{3}}{2 T_{0}}(w \cdot \operatorname{grad} \bar{\theta}+\bar{w} \cdot \operatorname{grad} \theta)+\sigma_{2} \gamma|\operatorname{grad}(\omega)|^{2}- \\
& -b_{0} b_{0}(\operatorname{grad} \bar{v} \cdot \operatorname{rot} \omega+\operatorname{grad} \cdot \operatorname{rot} \bar{\omega}] d x=0
\end{aligned}
$$

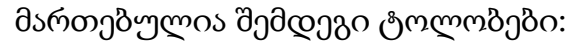


1)

$$
\begin{aligned}
& 2 x|\omega|^{2}-x(\omega r o t \bar{u}+\bar{\omega} \text { rotu })+\frac{x}{2}|\operatorname{rotu}|^{2}= \\
& =2 x\left|\omega-\frac{1}{2} r o t u\right|^{2} \geq 0,
\end{aligned}
$$

2)

$$
\begin{aligned}
& \varkappa_{2}|w|^{2}+\frac{\varkappa_{1}+T_{0} \varkappa_{3}}{2 T_{0}}(w \cdot \operatorname{grad} \bar{\theta}+\bar{w} \cdot \operatorname{grad} \theta)+\frac{\varkappa_{7}}{T_{0}}|\operatorname{gad} \theta|^{2}= \\
& =\frac{4 \varkappa_{2} \varkappa_{7} T_{0}-\left(\varkappa_{1}+T_{0} \varkappa_{3}\right)^{2}}{4 T_{0} \varkappa_{7}}|w|^{2}+\frac{1}{4 T_{0} \varkappa_{7}}\left|2 \varkappa_{7} \operatorname{grad} \theta+\left(\varkappa_{1}+T_{0} \varkappa_{3}\right) w\right|^{2} \geq 0,
\end{aligned}
$$

3)

$$
\begin{aligned}
& \frac{2 \lambda+2 \mu+x}{2}|\operatorname{divu}|^{2}+\mu_{0}(\operatorname{vdiv} \bar{u}+\bar{v} \operatorname{div} u)+\eta|v|^{2}= \\
& =\frac{(2 \lambda+2 \mu+x) \eta-2 \mu_{0}^{2}}{2}|\operatorname{divu}|^{2}+\frac{1}{\eta}\left|\eta v+\mu_{0} \operatorname{divu}\right|^{2} \geq 0,
\end{aligned}
$$

4)

$$
\begin{aligned}
& a_{0}|\operatorname{grad} v|^{2}-b_{0}(\operatorname{grad} v \cdot \operatorname{rot} \bar{\omega}+\operatorname{grad} \bar{\nu} \operatorname{rot} \omega)+ \\
& +\left.\gamma \operatorname{grad} \omega\right|^{2}=\frac{a_{0} \gamma-b_{0}^{2}}{a_{0}}|\operatorname{rot} \omega|^{2}+\frac{1}{a_{0}}\left|a_{0} \operatorname{grad} v+b_{0} \operatorname{rot} \omega\right|^{2} \geq 0,
\end{aligned}
$$

sન зовงณ

$$
\begin{gathered}
|\operatorname{grad} \omega|^{2}=|\operatorname{rot} \omega|^{2}, \\
E^{(1)}(\bar{u}, u)=\frac{2 \lambda+2 \mu+x}{2}|\operatorname{div} u|^{2}+\frac{\varkappa}{2}|\operatorname{rotu}|^{2}+\widetilde{E}^{(1)}(\bar{u}, u),
\end{gathered}
$$

$\operatorname{bscos} 3$

$$
\widetilde{E}^{(1)}(\bar{u}, u)=\frac{2 \mu+\varkappa}{2}\left[\left|\frac{\partial u_{2}}{\partial x_{1}}+\frac{\partial u_{1}}{\partial x_{2}}\right|^{2}+\left|\frac{\partial u_{1}}{\partial x_{1}}-\frac{\partial u_{2}}{\partial x_{2}}\right|^{2}\right]
$$

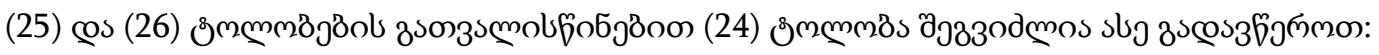




$$
\begin{aligned}
& \int_{\Omega^{ \pm}}\left[\sigma_{2} \widetilde{E}^{(1)}(\bar{u}, u)+E^{(2)}(\bar{w}, w)+\rho \sigma_{2}|\sigma|^{2}|u|^{2}+\sigma_{2} b|w|^{2}+\right. \\
& +\sigma_{2}|\sigma|^{2} I_{1}|\omega|^{2}+\sigma_{2}|\sigma|^{2} I|v|^{2}+\frac{\sigma_{2} c}{T_{0}}|\theta|^{2}+ \\
& +2 \varkappa \sigma_{2}\left|\omega-\frac{1}{2} \operatorname{rotu}\right|^{2}+\frac{4 \varkappa_{2} \varkappa_{7} T_{0}-\left(\varkappa_{1}+T_{0} \varkappa_{3}\right)^{2}}{4 T_{0} \varkappa_{7}} \mid w^{2}+ \\
& +\frac{1}{4 T_{0} \varkappa_{7}}\left|2 \varkappa_{7} \operatorname{grad} \theta+\left(\varkappa_{1}+T_{0} \varkappa_{3}\right) w\right|^{2}+\frac{\sigma_{2}}{\eta}\left|\eta v+\mu_{0} \operatorname{divu}\right|^{2} \\
& +\sigma_{2} \frac{(2 \lambda+2 \mu+x) \eta-2 \mu_{0}^{2}}{2 \eta}|\operatorname{divu}|^{2}+\sigma_{2} \frac{a_{0} \gamma-b_{0}^{2}}{a_{0}}|\operatorname{rot} \omega|^{2}+ \\
& +\frac{\sigma_{2}}{a_{0}}\left|a_{0} \operatorname{gradv}+b_{0} \operatorname{rot} \omega\right|^{2} d x=0
\end{aligned}
$$

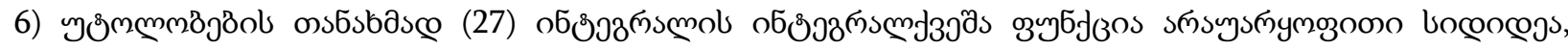

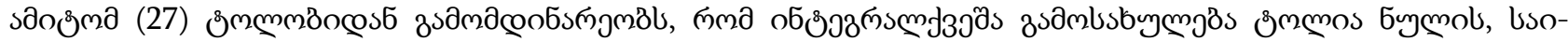

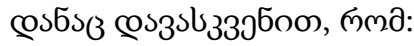

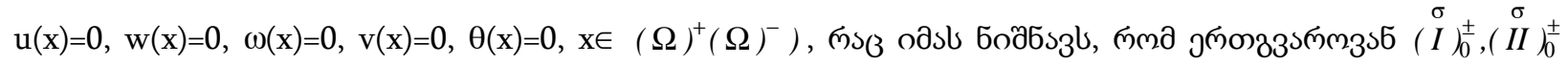

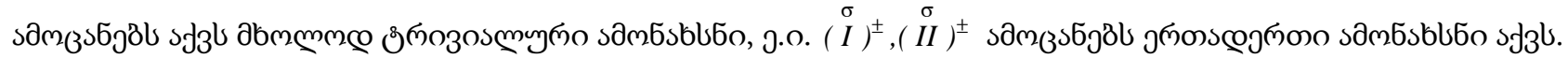

\section{cosb $33^{6 s}$}

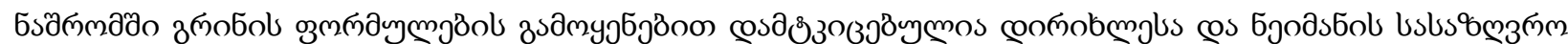

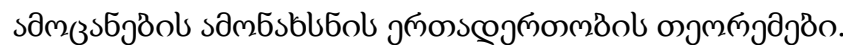

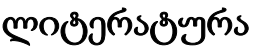

1. Iesan D. Thermoelastisity of bodies with microstructure and microtemperatures. International journal of solids and structures. 44(2007) 8648-8662. 2007.

2. Iesan D. On a theory of micromorfic elastic solids with microtemperatures. Journal of thermal stresses. 24(8). 2001.

3. Giorgashvili L., Zazashvili S., Mathematical problems of thermoelasticity of bodies with microstructure and microtemperature. Transactions of A. Razmadze Mathematical Institute. V.172. Issue 1. 2018, 30-57 pp. (in Georgian).

4. Bitsadze L., Jaiani G. Theorems for the third and fourth BVPs of 2D theory of thermoelasticity with microtemperatures. Nova Science Publishers, Inc. QA431.M.36. 2012, 99-118 pp. 
UDC 621.397.2

SCOPUS CODE 2610

DOI: https://doi.org/10.36073/1512-0996-2019-3-111-120

The Uniqueness Theorem of the boundary value problems for the stationary oscilations of the Theory of Thermoelasticity

Tinatin Kapanadze Department of Mathematics, Georgian Technical University, 77 M. Kostava str, 0160 Tbilisi, Georgia

E-mail: tinatin.kapanaZe@gmail.com

\section{Reviewers:}

S. Kharibegashvili, Professor, Faculty of Informatics and Control Systems, GTU

E-mail: kharibegashvili@yahoo.com

L. Bitsadze, Research Scientist, Ilia Vekua Institute of Applied Mathematics of Ivane Javakhishvili Tbilisi State University

E-mail: lamarabits@yahoo.com

Abstract. We consider the stationary oscillations of the micro-stretch materials with microstructure and microtemperatures. The representation formula of a general solution of the homogeneous system of differential equations obtained in the paper is expressed by means of seven metaharmonic functions. These formulas are very convenient in many particular problems for domains with concrete geometry.

Key words: Microtemperature; oscillation frequency; pseudo oscillation; stationary oscillation. 
UDC 621.397.2

SCOPUS CODE 2610

DOI: https://doi.org/10.36073/1512-0996-2019-3-111-120

\section{Теорема единственности решений граничных задач стационарного колебания теории термоупругости}

Тинатин Капанадзе Департамент математики, Грузинский технический университет, Грузия, 0160, Тбилиси, ул. М. Костава, 77

E-mail: tinatin.kapanaZe@gmail.com

\section{Рецензенты:}

С. Харибегашвили, профессоор факультета информатики и систем управления ГТУ E-mail: kharibegashvili@yahoo.com

Л. Бицадзе, научный сотрудник Института прикладной математики им. И. Векуа

E-mail: lamarabits@yahoo.com

Аннотация. В работе рассматриваются основные граничные задачи стационарных колебаний теории термоупругости (псевдо -колебаний, когда частота колебаний $\sigma_{=} \sigma_{1}+\mathrm{i} \sigma_{2} . \sigma_{2}>0, \sigma_{1} \in \mathrm{R}$ ), когда на границе задано предельное значение вектора $U=(u, w, \omega, v, \theta)^{\top}$ (задача Дирихле), где $u(x)$-вектор перемещения, $w=$ $\left(w_{1}, w_{2}\right)^{\top}$ - вектор микротемпературы, $\omega(x)$ - функция микровращений, $v(x)$ - функция микрорастяжений, $\theta(x)$ - функция температуры тела. В случае задачи Неймана на границе задано предельное значение обобщенного термонапряжения.

В работе получены формулы Грина для системы однородных дифференциальных уравнений. C помощью этих формул доказаны теоремы единственности граничных задач Дирихле и Неймана. В частности доказано, что, если задача Дирихле и Неймана имеют решение, то оно единственно.

Ключевые слова: микротемпература; псевдоколебание; стационарное колебание; частата колебаний.

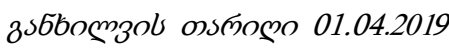

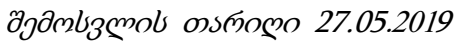

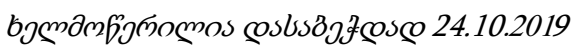




\section{UDC 536.7}

SCOPUS CODE 2610

DOI: https://doi.org/10.36073/1512-0996-2019-3-121-132

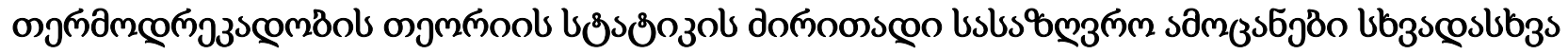

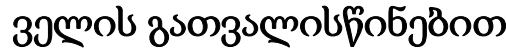

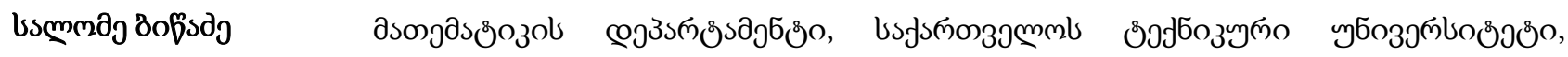

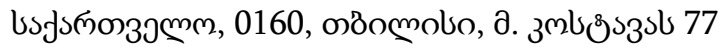

E-mail: Sali.bitsadze28@gmail.com

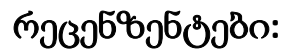

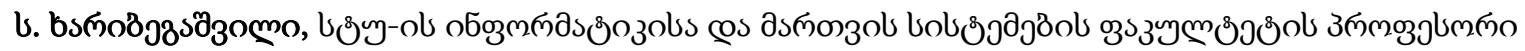

E-mail: kharibegashvili@yahoo.com

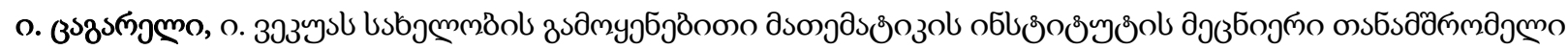

E-mail: I.Tsagareli@yahoo.com

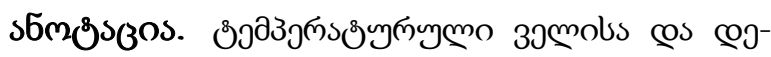

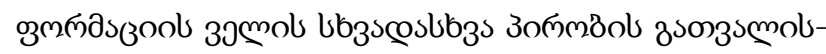

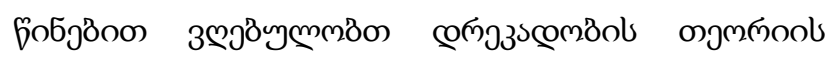

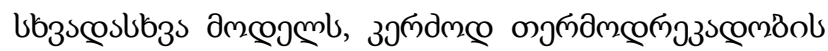

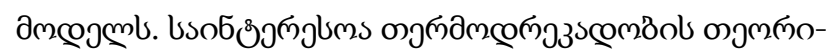

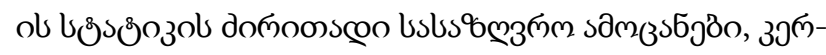

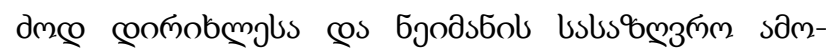

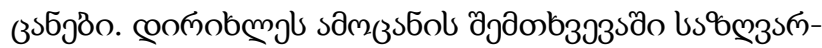

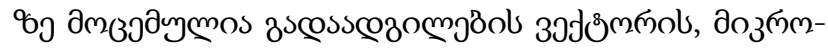

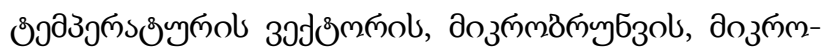

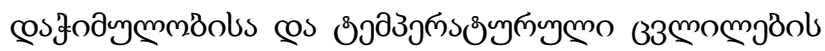

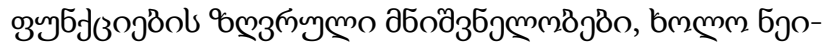

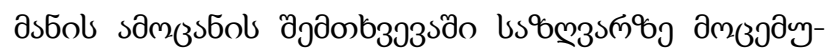

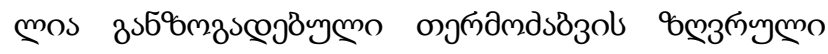

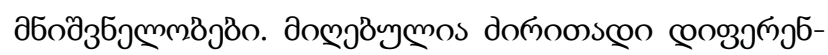

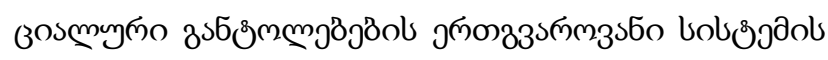

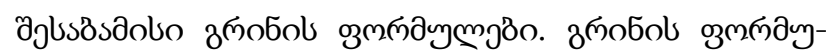

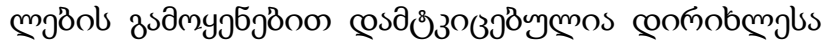

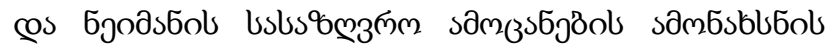

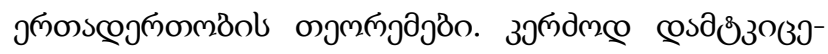

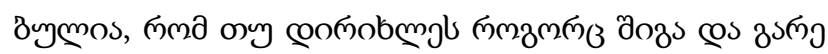

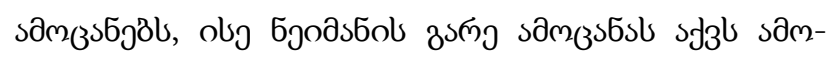

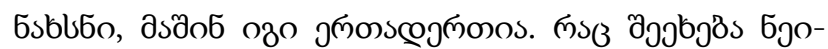

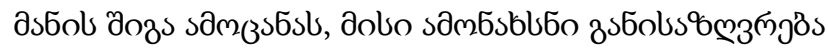

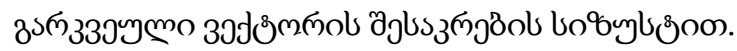

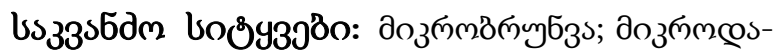

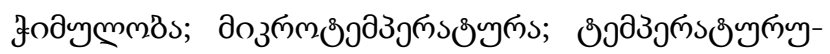
mo 33 momgls.

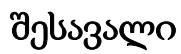

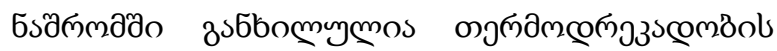

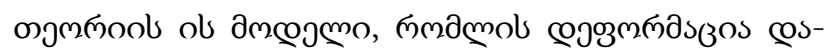

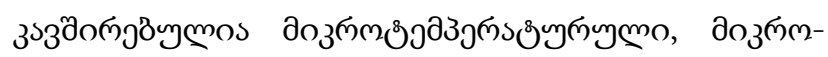




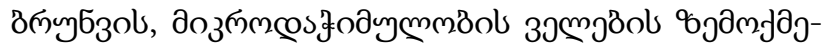

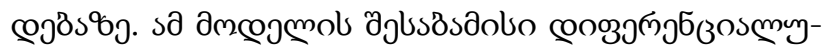

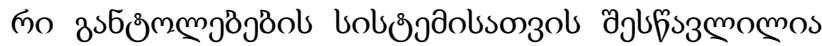

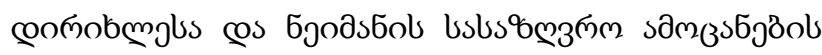

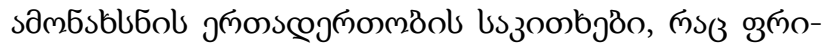

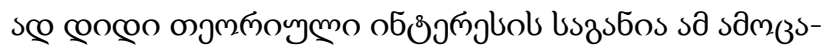

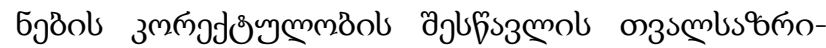

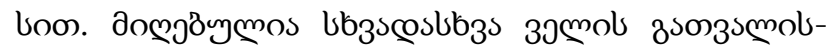

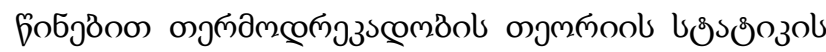

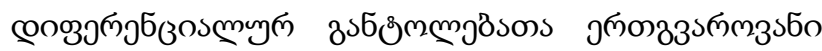

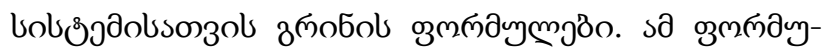

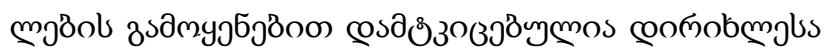

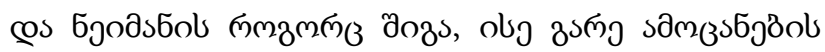

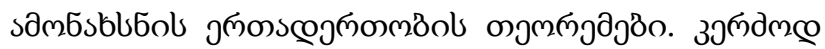

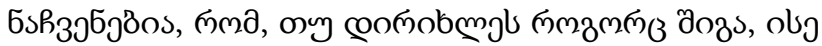

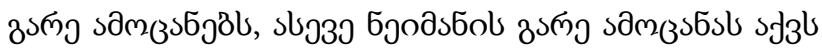

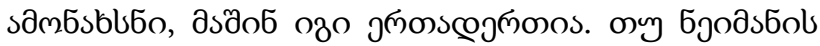

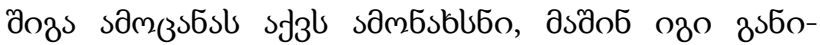

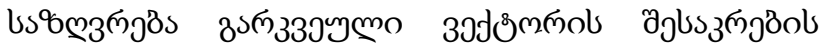

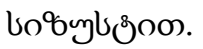

\section{donoonsco 6sfomo}

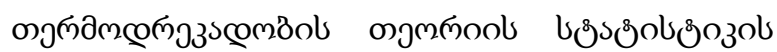

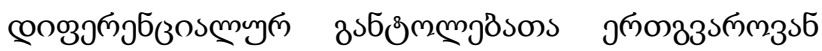

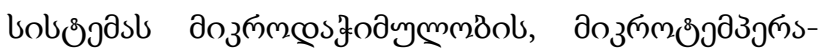

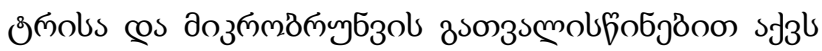

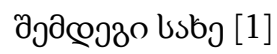

$$
\begin{gathered}
(\mu+\varkappa) \Delta u+(\lambda+\mu) \text { graddivu }-\varkappa r o t \omega+\mu_{0} \operatorname{grad} v-\beta_{0} \operatorname{grad} \theta=0, \\
\varkappa_{6} \Delta w-\varkappa_{2} w+\left(\varkappa_{4}+\varkappa_{5}\right) \text { graddivw }-\varkappa_{3} \operatorname{grad} \theta=0, \gamma \Delta \omega-2 \varkappa \omega+\varkappa \text { rotu }-\mu_{1} \text { rotw }=0, \\
a_{0} \Delta v-\eta v-\mu_{0} \text { divu }-\mu_{2} \operatorname{div} w+\beta_{1} \theta=0, \varkappa_{7} \Delta \theta+\varkappa_{1} \text { divw }=0,
\end{gathered}
$$

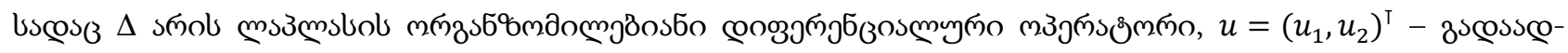

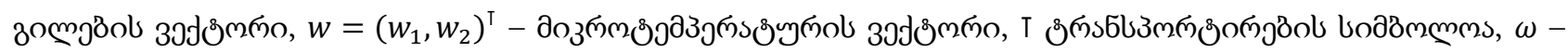

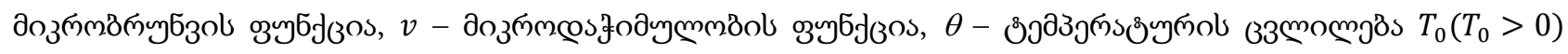

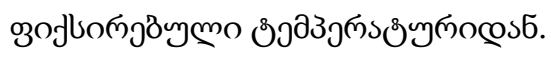

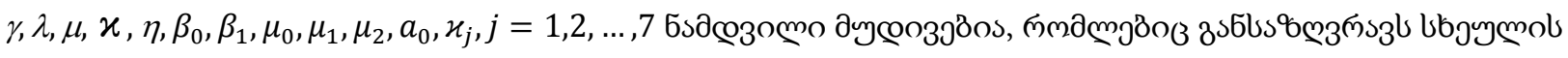

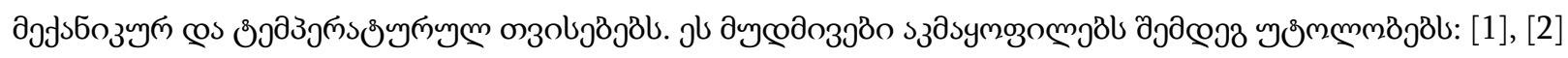

$$
\begin{gathered}
a_{0}>0, \mu>0, \eta>0, \gamma>0, \varkappa>0,2 \lambda+2 \mu+\varkappa-2 \mu_{0}^{2}>0, a_{0} \gamma-b_{0}^{2}>0, \varkappa_{7}>0,\left(\varkappa_{1}+\varkappa_{3} T_{0}\right)^{2} \leq \\
4 T_{0} \varkappa_{2} \varkappa_{7}, \varkappa_{6} \pm \varkappa_{5} \geq 0,2 \varkappa_{4}+\varkappa_{5}+\varkappa_{6}>0 .
\end{gathered}
$$

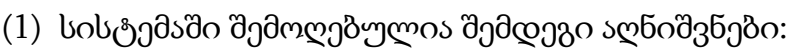

$$
\begin{gathered}
r o t:=\left(-\frac{\partial}{\partial x_{2}}, \frac{\partial}{\partial x_{1}}\right)^{\top}, \operatorname{rot} \omega:=\left(-\frac{\partial \omega}{\partial x_{2}}, \frac{\partial \omega}{\partial x_{1}}\right)^{\top} \\
r o t u:=\frac{\partial u_{2}}{\partial x_{1}}-\frac{\partial u_{1}}{\partial x_{2}}, \operatorname{rot} w=\frac{\partial w_{2}}{\partial x_{1}}-\frac{\partial w_{1}}{\partial x_{2}} .
\end{gathered}
$$




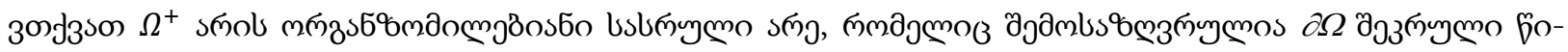
๓о口, $\Omega^{-}=R^{2} \backslash \bar{\Omega}^{+}$.

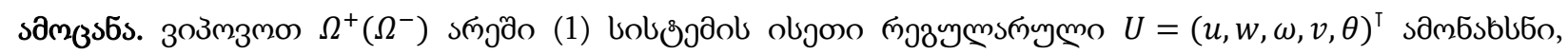

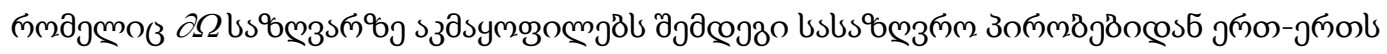

(I) (comgobmgls sam(zsбs):

$$
\{U(z)\}^{+}=f(z),\left(\{U(z)\}^{-}=f(z)\right)
$$

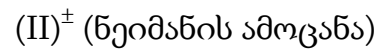

$$
\{P(\partial, n) U(z)\}^{+}=f(z),\left(\{P(\partial, n) U(z)\}^{-}=f(z)\right)
$$

$\operatorname{sicos} 3$

$$
f=\left(f^{(1)}, f^{(2)}, f_{3}, f_{4}, f_{5}\right)^{\top}, f^{(j)}=\left(f_{1}^{(j)}, f_{2}^{(j)}\right)^{\top}, j=1,2,
$$

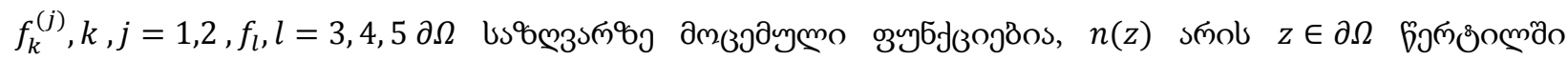

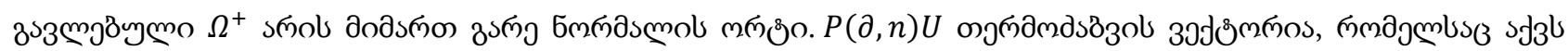

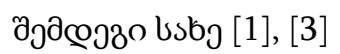

$$
P(\partial, n) U=\left(T^{(1)}(\partial, n) U, T^{(2)}(\partial, n) U, T^{(3)}(\partial, n) U, T^{(4)}(\partial, n) U, T^{(5)}(\partial, n) U\right)^{\top},
$$

bscosos

$$
\begin{gathered}
T^{(1)}(\partial, n) U:=(2 \mu+\mathcal{x}) \frac{\partial u}{\partial n}+\lambda n \text { divu }-(\mu \text { rotu }+\varkappa \omega) s+\left(\mu_{0} v-\beta_{0} \theta\right) n, \\
T^{(2)}(\partial, n) U:=\left(\varkappa_{5}+\varkappa_{6}\right) \frac{\partial w}{\partial n}+\varkappa_{4} n \text { divw }-\varkappa_{5} \text { s rotw }, \\
T^{(3)}(\partial, n) U:=\gamma \frac{\partial \omega}{\partial n}-\mu_{1}(s \cdot w)-b_{0}(s \cdot \operatorname{grad} v), \\
T^{(4)}(\partial, n) U:=a_{0} \frac{\partial v}{\partial n}-\mu_{2}(n \cdot w)+b_{0}(s \cdot \operatorname{grad} \omega), \\
T^{(5)}(\partial, n) U:=\mu_{7} \frac{\partial \theta}{\partial n}+\varkappa_{1}(n \cdot w) .
\end{gathered}
$$

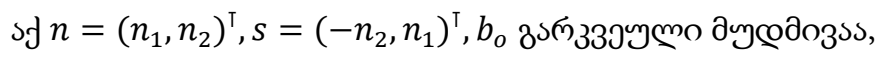

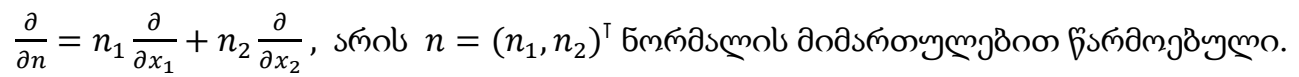

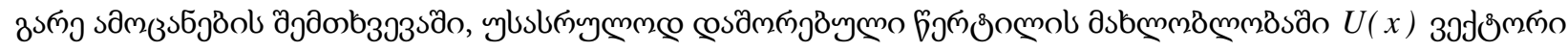

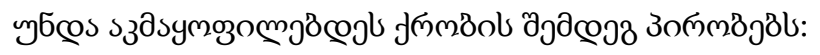

$$
U(x)=O\left(|x|^{-1}\right), \partial_{k} U(x)=o\left(|x|^{-1}\right), k=1,2 .
$$

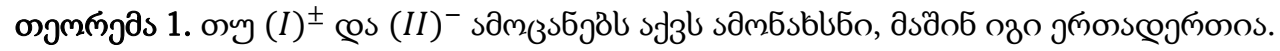

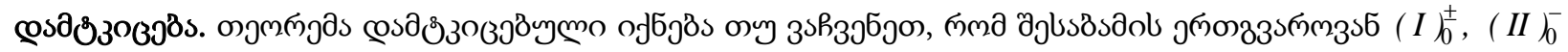

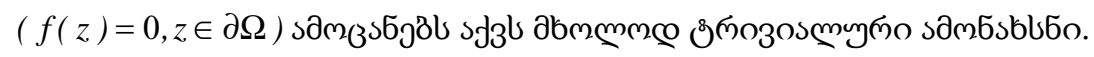




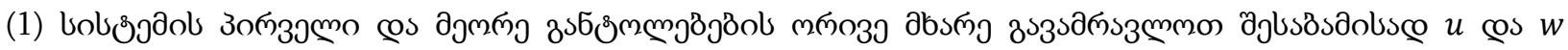

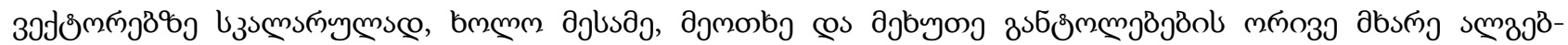

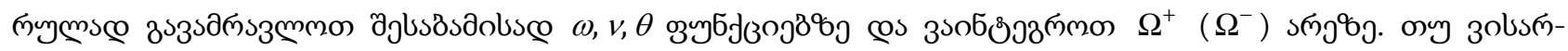

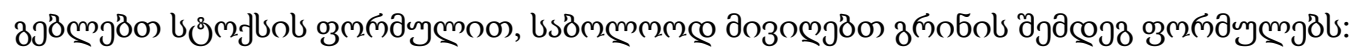

$$
\begin{aligned}
& \quad \pm \int_{\partial \Omega}\left\{u(z) \cdot T^{(1)}(\partial, n) U(z)\right\}^{ \pm} d s-\int_{\Omega^{ \pm}}\left[E^{(1)}(u, u)+\mu_{0} v d i v u-\right. \\
& \left.\quad-\beta_{0} \theta d i v u-x \omega r o t u\right] d x=0, \\
& \quad \pm \int_{\partial \Omega}\left\{w(z) \cdot T^{(2)}(\partial, n) U(z)\right\}^{ \pm} d s-\int_{\Omega^{ \pm}}\left[E^{(2)}(w, w)+x_{2} w^{2}+\right. \\
& \left.\quad+x_{3} w \cdot \operatorname{grad} \theta\right] d x=0, \\
& \quad \pm \int_{\partial \Omega}\left\{\omega(z) \cdot T^{(3)}(\partial, n) U(z)\right\}^{ \pm} d s-\int_{\Omega^{ \pm}}\left[\gamma(\operatorname{grad} \omega)^{2}+2 x \omega^{2}-\right. \\
& \left.\quad-x \omega \operatorname{rotu}-\mu_{1} w \cdot \operatorname{rot} \omega-b_{0} \operatorname{grad} v \cdot \operatorname{rot} \omega\right] d x=0, \\
& \quad \pm \int_{\partial \Omega}\left\{v(z) \cdot T^{(4)}(\partial, n) U(z)\right\}^{ \pm} d s-\int_{\Omega^{ \pm}}\left[a_{0}(\operatorname{grad} v)^{2}+\eta v^{2}+\right. \\
& \left.\quad+\mu_{0} v \operatorname{divu}-\beta_{1} v \theta-\mu_{2} w \cdot \operatorname{grad}-b_{0} \operatorname{gradv} \cdot \operatorname{rot} \omega\right] d x=0, \\
& \pm \int_{\partial \Omega}\left\{\theta(z) \cdot T^{(5)}(\partial, n) U(z)\right\}^{ \pm} d s-\int_{\Omega^{ \pm}}\left[x_{7}(\operatorname{grad} \theta)^{2}+x_{1} w \cdot \operatorname{grad} \theta\right] d x=0,
\end{aligned}
$$

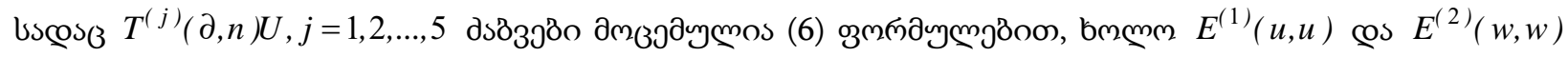

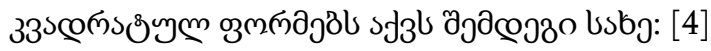

$$
\begin{aligned}
& E^{(1)}(u, u)=\left(\lambda+\mu+\frac{x}{2}\right)(\operatorname{divu})^{2}+\frac{2 \mu+x}{2}\left[\left(\frac{\partial u_{2}}{\partial x_{1}}+\frac{\partial u_{1}}{\partial x_{2}}\right)^{2}+\right. \\
& \left.+\left(\frac{\partial u_{1}}{\partial x_{1}}-\frac{\partial u_{2}}{\partial x_{2}}\right)^{2}\right]+\frac{x}{2}(\text { rotu })^{2}, \\
& E^{(2)}(w, w)=\frac{2 x_{4}+x_{5}+x_{6}}{2}(\operatorname{div} w)^{2}+\frac{x_{5}+x_{6}}{2}\left[\left(\frac{\partial w_{1}}{\partial x_{2}}+\frac{\partial w_{2}}{\partial x_{1}}\right)^{2}+\right. \\
& \left.+\left(\frac{\partial w_{1}}{\partial x_{1}}-\frac{\partial w_{2}}{\partial x_{2}}\right)\right]^{2}+\frac{x_{6}-x_{5}}{2}(\text { rotw })^{2} .
\end{aligned}
$$

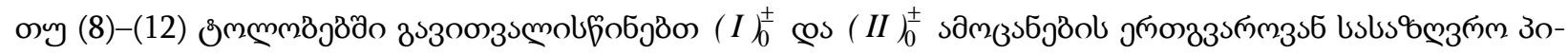

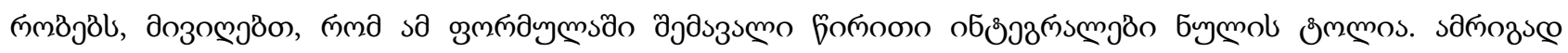

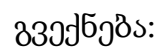

$$
\int_{\Omega^{ \pm}}\left[E^{(1)}(u, u)+\mu_{0} v \operatorname{div} u-\beta_{0} \theta \operatorname{div} u-\varkappa \omega r o t u\right] d x=0,
$$




$$
\begin{gathered}
\int_{\Omega^{ \pm}}\left[E^{(2)}(w, w)+\varkappa_{2} w^{2}+x_{3} w \cdot \operatorname{grad} \theta\right] d x=0, \\
\int_{\Omega^{ \pm}}\left[\gamma(\operatorname{grad} \omega)^{2}+2 x \omega^{2}-x \omega r o t u-\mu_{1} w \cdot \operatorname{rot} \omega-b_{0} \operatorname{grad} v \cdot \operatorname{rot} \omega\right] d x=0, \\
\int_{\Omega^{ \pm}}\left[a_{0}(\operatorname{grad} v)^{2}+\eta v^{2}+\mu_{0} v d i v u-\beta_{1} v \theta-\mu_{2} w \cdot \operatorname{grad} v-\right. \\
\left.-b_{0} \operatorname{grad} v \cdot \operatorname{rot} \omega\right] d x=0, \\
\int_{\Omega^{ \pm}}\left[x_{7}(\operatorname{grad} \theta)^{2}+\varkappa_{1} w \cdot \operatorname{grad} \theta\right] d x=0 .
\end{gathered}
$$

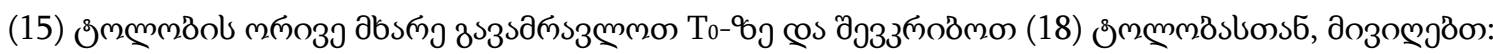

$$
\begin{aligned}
& \int_{\Omega^{ \pm}}\left[T_{0} E^{(2)}(w, w)+\varkappa_{2} T_{0} w^{2}+\left(\varkappa_{1}+T_{0} \varkappa_{3}\right)(w \cdot \operatorname{grad} \theta)+\right. \\
& \left.+\chi_{7}(\operatorname{grad} \theta)^{2}\right] d x=0 .
\end{aligned}
$$

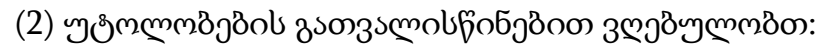

$$
\begin{aligned}
& \varkappa_{2} T_{0} w^{2}+\left(x_{1}+T_{0} \varkappa_{3}\right)(w \cdot \operatorname{grad} \theta)+\varkappa_{7}(\operatorname{grad} \theta)^{2}= \\
& =\frac{4 T_{0} \varkappa_{2} x_{7}-\left(\varkappa_{1}+T_{0} \varkappa_{3}\right)^{2}}{4 x_{7}} w^{2}+\frac{1}{4 x_{7}}\left[\left(x_{1}+T_{0} \varkappa_{3}\right) w+2 x_{7} \operatorname{grad} \theta\right]^{2} \geq 0 .
\end{aligned}
$$

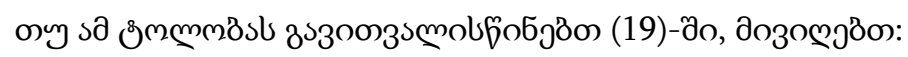

$$
\begin{aligned}
& \int_{\Omega^{ \pm}}\left[T_{0} E^{(2)}(w, w)+\frac{4 T_{0} \varkappa_{2} \varkappa_{7}-\left(\varkappa_{1}+T_{0} \varkappa_{3}\right)^{2}}{4 \chi_{7}} w^{2}+\right. \\
& \left.+\frac{1}{4 \varkappa_{7}}\left(\left(\varkappa_{1}+T_{0} \varkappa_{3}\right) w+2 \varkappa_{7} \operatorname{grad} \theta\right)^{2}\right] d x=0 .
\end{aligned}
$$

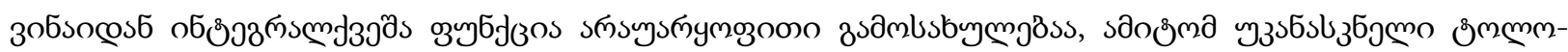

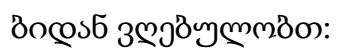

$$
E^{(2)}(w, w .)=0, w(x)=0, \operatorname{grad} \theta(x)=0, x \in \Omega^{ \pm} .
$$

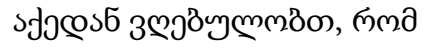

$$
w(x)=0, \theta(x)=c=\text { const, } x \in \Omega^{ \pm} .
$$

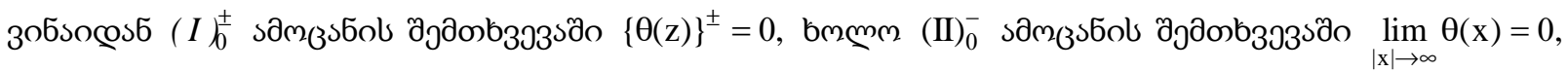

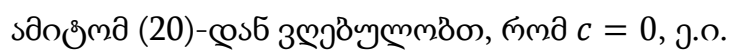

$$
w(x)=0, \quad \theta(x)=0, x \in \Omega^{ \pm} .
$$

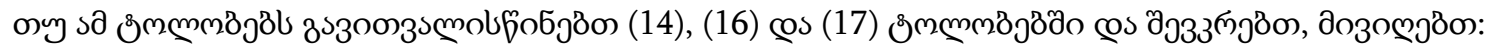

$$
\begin{aligned}
& \int_{\Omega^{ \pm}}\left[E^{(1)}(u, u)+\gamma(\operatorname{grad} \omega)^{2}+a_{0}(\operatorname{grad} v)^{2}+2 x \omega^{2}+\right. \\
& \left.+\eta v^{2}+2 \mu_{0} v d i v u-2 \varkappa \omega \text { rotu }-2 b_{0} \text { grad } \cdot \operatorname{rot} \omega\right] d x=0 .
\end{aligned}
$$




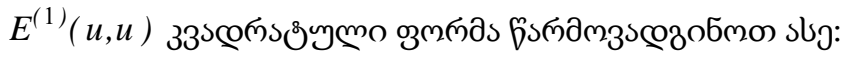

$$
E^{(1)}(u, u)=\tilde{E}^{(1)}(u, u)+\frac{\varkappa}{2}(r o t u)^{2}+\left(\lambda+\mu+\frac{\varkappa}{2}\right)(\operatorname{div} u)^{2},
$$

bscoso

$$
\widetilde{E}^{(1)}(u, u)=\frac{2 \mu+\varkappa}{2}+\left[\left(\frac{\partial u_{2}}{\partial x_{1}}+\frac{\partial u_{1}}{\partial x_{2}}\right)^{2}+\left(\frac{\partial u_{1}}{\partial x_{1}}-\frac{\partial u_{2}}{\partial x_{2}}\right)^{2}\right] \geq 0 .
$$

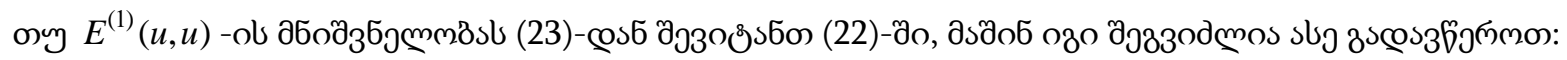

$$
\begin{aligned}
& \int_{\Omega^{ \pm}}\left\{\tilde{E}^{(1)}(u, u)+2 \varkappa\left(\omega-\frac{1}{2} \operatorname{rotu}\right)^{2}+\right. \\
& +\left[\left(\lambda+\mu+\frac{x}{2}\right)(\operatorname{div} u)^{2}+2 \mu_{0} v \operatorname{divu}+\eta v^{2}\right]+ \\
& \left.+\left[a_{0}(\operatorname{grad} v)^{2}-2 b_{0}(\operatorname{grad} v \cdot \operatorname{rot} \omega)+\gamma(\operatorname{rot} \omega)^{2}\right]\right\} d x=0 .
\end{aligned}
$$

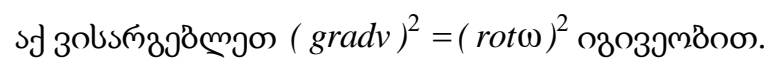

әЭЗбоðбмо, ґма

$$
\begin{gathered}
\left(\lambda+\mu+\frac{x}{2}\right)(\operatorname{divu})^{2}+2 \mu_{0} v \operatorname{div} u+\eta v^{2}=\frac{(2 \lambda+2 \mu+x) \cdot \eta-2 \mu_{0}^{2}}{2 \eta}(\operatorname{divu})^{2}+ \\
+\frac{1}{\eta}\left(\mu_{0} \operatorname{divu}+\eta v\right)^{2} \geq 0, \\
a_{0}(\operatorname{grad} v)^{2}-2 b_{0}(\operatorname{grad} v \cdot \operatorname{rot} \omega)+\gamma(\operatorname{rot} \omega)^{2}= \\
=\frac{a_{0} \gamma-b_{0}^{2}}{a_{0}}(\operatorname{rot} \omega)^{2}+\frac{1}{a_{0}}\left(b_{0} \operatorname{rot} \omega-a_{0} \operatorname{grad} v\right)^{2} \geq 0 .
\end{gathered}
$$

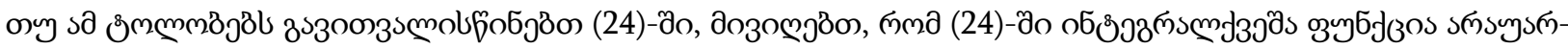

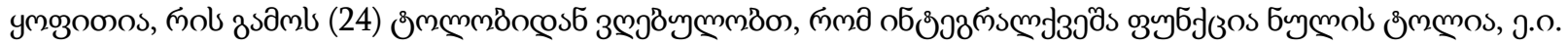

$$
\widetilde{E}^{(1)}(u, u)=0, \omega-\frac{1}{2} r o t u=0, \operatorname{div} u=0, \operatorname{rot} \omega=0, v=0,
$$

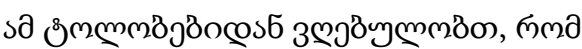

$$
u(x)=b \tilde{x}+d, \omega(x)=b, v(x)=0, \quad x \in \Omega^{ \pm},
$$

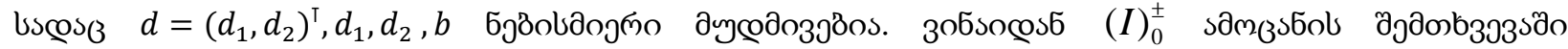

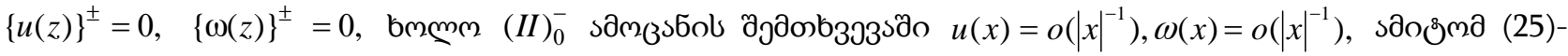

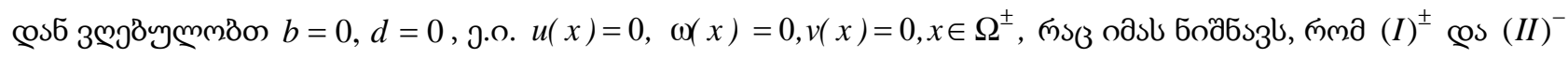

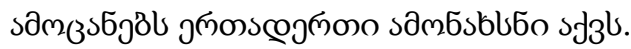




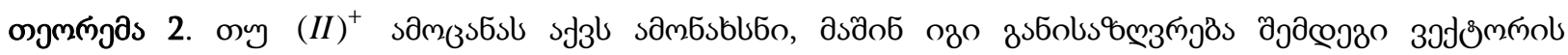
bo6ylson:

$$
U^{(0)}(x)=\left(b \tilde{x}+p^{\prime} c x+d, 0, b, q^{\prime} c, c\right)^{\top},
$$

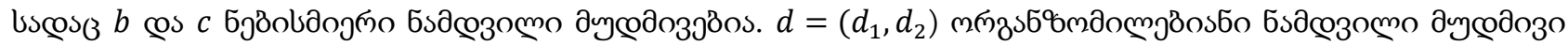

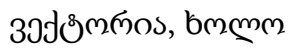

$$
p^{\prime}=\frac{\eta \beta_{0}-\mu_{0} \beta_{1}}{(2 \lambda+2 \mu+\varkappa) \eta-2 \mu_{0}^{2}}, q^{\prime}=\frac{\beta_{1}(2 \lambda+2 \mu+\varkappa)-2 \mu_{0} \beta_{0}}{(2 \lambda+2 \mu+\varkappa) \eta-2 \mu_{0}^{2}}
$$

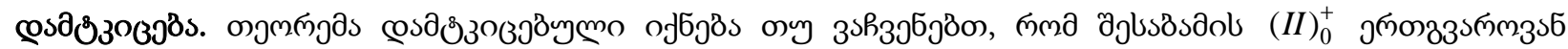

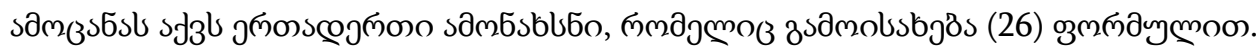

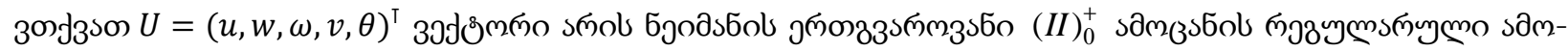

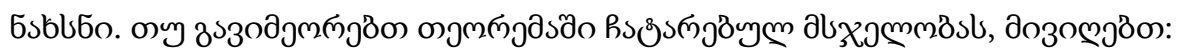

$$
w(x)=0, \theta(x)=c=\text { const }, \quad x \in \Omega^{+},
$$

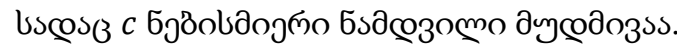

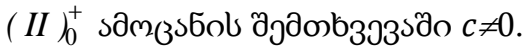

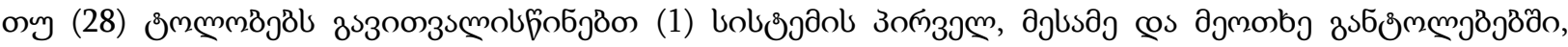
дозомวठัо:

$$
\begin{gathered}
(\mu+\varkappa) \Delta u+(\lambda+\mu) \text { graddivu }+\varkappa \operatorname{rot} \omega+\mu_{0} \operatorname{grad} v=0, \\
\lambda \Delta \omega-2 \varkappa \omega+\mathcal{\varkappa r o t u}=0, \\
a_{0} \Delta v-\eta v-\mu_{0} \operatorname{divu}+\beta_{1} c=0 .
\end{gathered}
$$

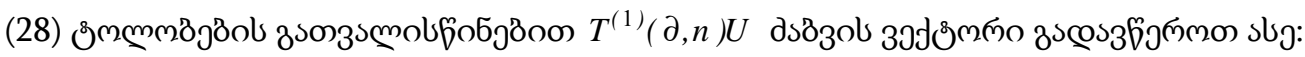

$$
T^{(1)}(\partial, n) U=(2 \mu+x) \frac{\partial u}{\partial n}+\lambda \text { ndivu }-\mu \text { srotu }-\varkappa s \omega+\left(\mu_{0} v-\beta_{0} c\right) n .
$$

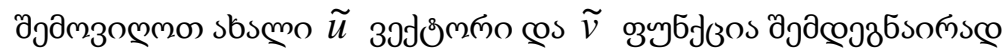

$$
\tilde{u}=u-p^{\prime} c x, \quad \tilde{v}=v-q^{\prime} c,
$$

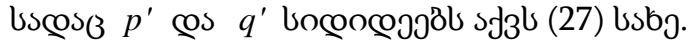

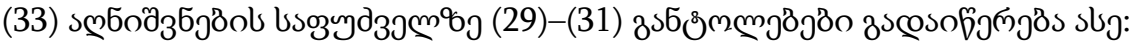

$$
\begin{gathered}
(\mu+x) \Delta \tilde{u}+(\lambda+\mu) \operatorname{graddiv} \tilde{u}-x \operatorname{rot} \omega+\mu_{0} \operatorname{grad} \tilde{v}=0, \\
\gamma \Delta \omega-2 x \omega+x \operatorname{rot} \tilde{u}=0, \\
a_{0} \Delta \tilde{v}-\eta \tilde{v}-\mu_{0} \tilde{u}=0 .
\end{gathered}
$$

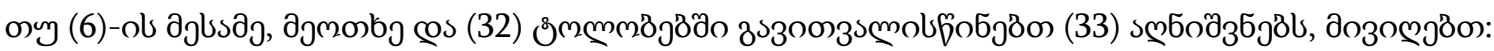




$$
\begin{gathered}
\left\{T^{(1)}(\partial, n) \tilde{U}(z)\right\}^{+}=\left\{(2 \mu+x) \frac{\partial \tilde{u}}{\partial n}+\lambda n d i v \tilde{u}-\mu s r o t \tilde{u}-\right. \\
\left.-x s \omega+\mu_{0} \tilde{v} n\right\}^{+}=0, z \in \partial \Omega, \\
\left\{T^{(3)}(\partial, n) \tilde{U}(z)\right\}^{+}=\left\{\gamma \frac{\partial \omega}{\partial n}-b_{0}(s \cdot \operatorname{grad} \tilde{v})\right\}^{+}=0, \quad z \in \partial \Omega, \\
\left\{T^{(4)}(\partial, n) \tilde{U}(z)\right\}^{+}=\left\{a_{0} \frac{\partial v}{\partial n}+b_{0}(s \cdot \operatorname{grad} \omega)\right\}^{+}=0, \quad z \in \partial \Omega, \\
\widetilde{U}=(\tilde{u}, \omega, \tilde{v})^{\top} .
\end{gathered}
$$

$\operatorname{sicos} 3$

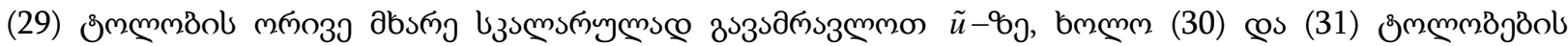

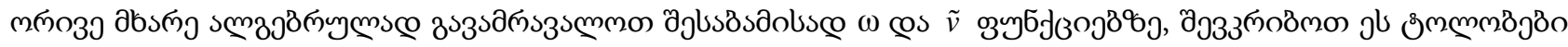

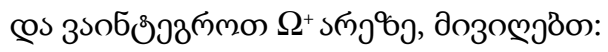

$$
\begin{aligned}
& \int_{\partial \Omega}\left\{\tilde{u}(z) T^{(1)}(\partial, n) \tilde{U}(z)+\omega(z) T^{(3)}(\partial, n) \tilde{U}(z)+\tilde{v}(z) T^{(4)}(\partial, n) \tilde{U}(z)\right\}^{+} d s- \\
& -\int_{\Omega+}\left[\tilde{E}^{(1)}(\tilde{u}, \tilde{u})+2 x\left(\omega-\frac{1}{2} \operatorname{rot} \tilde{u}\right)^{2}+\frac{(2 \lambda+2 \mu+x) \eta-2 \mu_{0}^{2}}{2 \eta}(\operatorname{div} \tilde{u})^{2}+\right. \\
& +\frac{1}{\eta}\left(\mu_{0} d i v \tilde{u}+\eta \tilde{v}\right)^{2}+\frac{a_{0} \gamma-b_{0}^{2}}{a_{0}}(\operatorname{rot} \omega)^{2}+\frac{1}{a_{0}}\left(b_{0} \operatorname{rot} \omega-\right. \\
& \left.\left.-a_{0} \operatorname{grad} \tilde{v}\right)^{2}\right] d x=0,
\end{aligned}
$$

$\operatorname{sicos} 3$

$$
\tilde{E}^{(1)}(\tilde{u}, \tilde{u})=\frac{2 \mu+x}{2}\left[\left(\frac{\partial \tilde{u}_{2}}{\partial x_{1}}+\frac{\partial \tilde{u}_{1}}{\partial x_{2}}\right)^{2}+\left(\frac{\partial \tilde{u}_{1}}{\partial x_{1}}-\frac{\partial \tilde{u}_{2}}{\partial x_{2}}\right)^{2}\right] .
$$

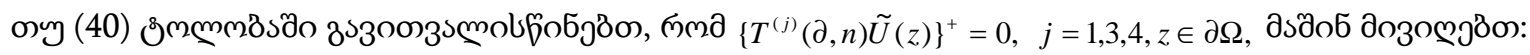

$$
\begin{aligned}
& \int_{\Omega+}\left[\tilde{E}^{(1)}(\tilde{u}, \tilde{u})+2 x\left(\omega-\frac{1}{2} \operatorname{rot} \tilde{u}\right)^{2}+\frac{(2 \lambda+2 \mu+x) \eta-2 \mu_{0}^{2}}{2 \eta}(\operatorname{div} \tilde{u})^{2}+\right. \\
& +\frac{1}{\eta}\left(\mu_{0} \operatorname{div} \tilde{u}+\eta \tilde{v}\right)^{2}+\frac{a_{0} \gamma-b_{0}^{2}}{a_{0}}(\operatorname{rot} \omega)^{2}+\frac{1}{a_{0}}\left(b_{0} \operatorname{rot} \omega-\right. \\
& \left.\left.-a_{0} \operatorname{grad} \tilde{v}\right)^{2}\right] d x=0 .
\end{aligned}
$$

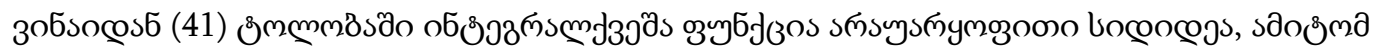

$$
\begin{aligned}
& \tilde{E}^{(1)}(\tilde{u}, \tilde{u})=0, \omega-\frac{1}{2} \operatorname{rot} \tilde{u}=0, \quad \operatorname{div} \tilde{u}=0, \tilde{v}=0, \\
& \operatorname{rot} \omega=0, \quad \operatorname{grad} \tilde{v}=0 .
\end{aligned}
$$

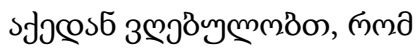

$$
u(x)=b \tilde{x}+d, \omega(x)=b, \tilde{v}(x)=0, x \in \Omega^{+} .
$$

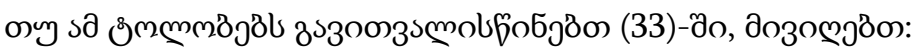

$$
u(x)=b \tilde{x}+d+p^{\prime} c x, \quad \omega(x)=b, \quad v(x)=q^{\prime} c,
$$

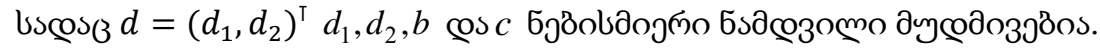




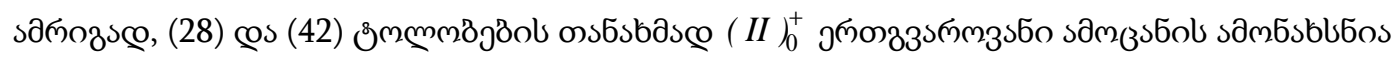

$$
U^{(0)}(x)=\left(b \tilde{x}+p^{\prime} c x+d, 0, b, q^{\prime} c, c\right)^{\top},
$$

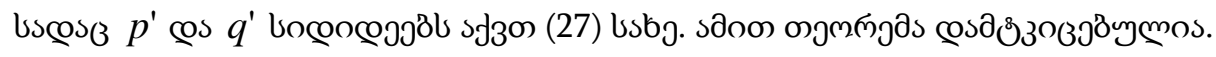

\section{$\cos 333^{6 s}$}

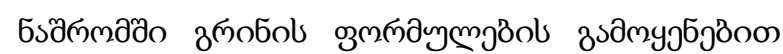

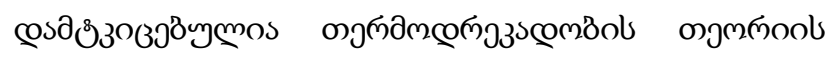

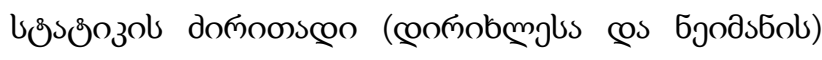

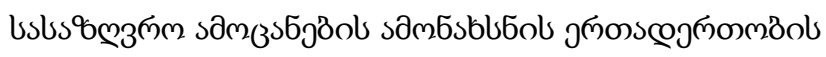
юэмпудјво.

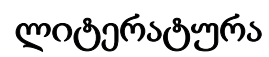

1. Iesan D. Thermoelastisity of bodies with microstructure and microtemperatures. International journal of solids and structures. 44(2007) 8648-8662. 2007.

2. Iesan D. On a theory of micromorfic elastic solids with microtemperatures. Journal of thermal stresses. 24(8). 2001.

3. Giorgashvili L., Zazashvili S., Mathematical problems of thermoelasticity of bodies with microstructure and microtemperature. Transactions of A. Razmadze Mathematical Institute. V.172. Issue 1. 2018, 30-57 pp. (in Georgian).

4. Bitsadze L., Jaiani G. Theorems for the third and fourth BVPs of 2D theory of thermoelasticity with microtemperatures. Nova Science Publishers, Inc. QA431.M.36. 2012, 99-118 pp.

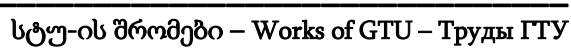

№3 (513), 2019 


\section{UDC 536.7}

SCOPUS CODE 2610

DOI: https://doi.org/10.36073/1512-0996-2019-3-121-132

\section{Basic boundary value problems of statics of Thermoelasticity theory considering different field}

Salome Bitsadze

Department of Mathematics, Georgian Technical University, 77 M. Kostava str, 0160 Tbilisi, Georgia

E-mail: Sali.bitsadze28@gmail.com

\section{Reviewers:}

S. Kharibegashvili, Professor, Faculty of Informatics and Control Systems, GTU

E-mail: kharibegashvili@yahoo.com

I. Tsagareli, Research Scientist, Ilia Vekua Institute of Applied Mathematics of Ivane Javakhishvili Tbilisi State University

E-mail: I.Tsagareli@yahoo.com

Abstract. The representation formula of a general solution of the homogeneous system of differential equations obtained in the paper is expressed by means of three harmonic and four metaharmonic functions. These formulas are very convenient and useful in many particular problems for domeins with concrete geometry.

Key words: Fugacity of temperature; metaharmonic; microrotation; microstretch; microtemperature. 


\title{
UDC 536.7
}

SCOPUS CODE 2610

DOI: https://doi.org/10.36073/1512-0996-2019-3-121-132

\section{Основные граничные задачи статики теории термоупругости с учётом разных полей}

Саломе Бицадзе

Департамент математики, Грузинский технический университет, Грузия, 0160, Тбилиси, ул. М. Костава, 77

E-mail: Sali.bitsadze28@gmail.com

\section{Рецензенты:}

С. Харибегашвили, профессор факультета информатики и систем управления ГТУ

E-mail: kharibegashvili@yahoo.com

И. Цагарели, научный работник Института математики им. И. Векуа

E-mail: I.Tsagareli@yahoo.com

Аннотация. В работе рассматриваются основные граничные задачи статики теории термоупругости, в частности граничные задачи Дирихле и Неймана. В случае задачи Дирихле на границе заданы предельные значения векторов перемещения и микротемпературы, а также функций микровращения, микрорастяжения и температуры. В случае задачи Неймана на границе задано предельное значение обобщенного термоупругого напряжения. Получены формулы Грина для системы однородных дифференциальных уравнений. С помощъю формулы Грина доказаны теоремы единственности граничных задач Дирихле и Неймана. В частности, если задача Дирихле, как внутренняя, так и внешняя, а также внешняя задача Неймана имеет решение, то оно единственно, что же касается решения внутренней задачи Неймана, то она определена с точностью до слагаемого определенного вектора.

Ключевые слова: микротемпература; микрорастяжения; микровращения; функция температуры.

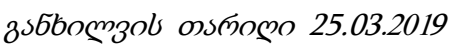

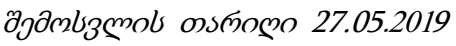

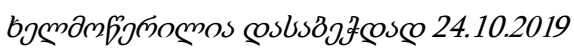




\title{
కЗరిmஙnos bsdojరిjmo
}

\section{Author's index}

\section{Указатель авторов}

\author{
s88\%os6odว œ. 29 \\ söbos6odg 3.29 \\ sбsбosðзоме b. 98

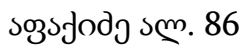

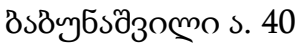 \\ Boffsdg ๒. 121

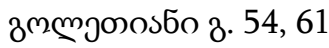

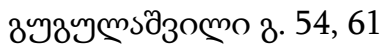 \\ obszsдg \%. 54, 61 \\ зรзумеов \%. 29 \\ зьбœ9мззио 6.11
}

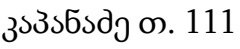

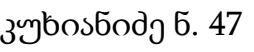

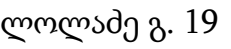

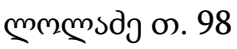

дmbsə̃ Зомо о. 40, 47, 68

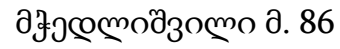

m50s6o ๖. 68

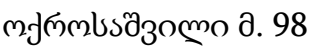

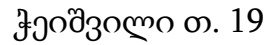

Махарадзе Л. 78 


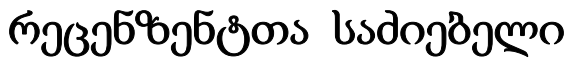

\section{Reviewer's index}

\section{Указатель рецензентов}

\begin{tabular}{|c|c|}
\hline 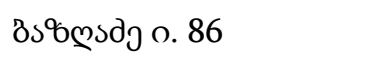 & 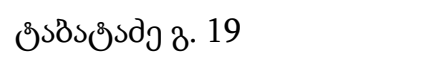 \\
\hline Bofதdว m. 111 & 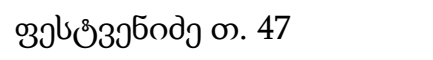 \\
\hline дмпূэmsd 3.19 & 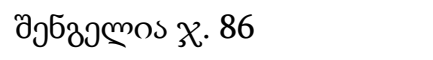 \\
\hline 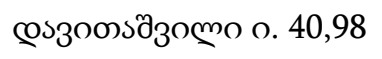 & 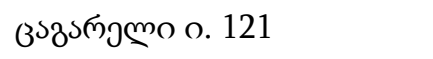 \\
\hline 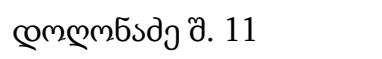 & 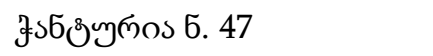 \\
\hline osðెంmo з. 11 & 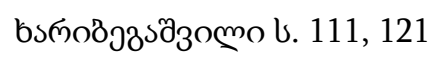 \\
\hline 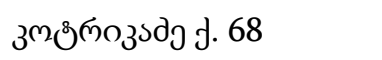 & 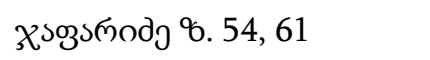 \\
\hline 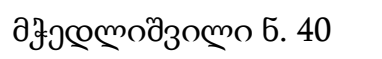 & žsogsmody 5. 29 \\
\hline 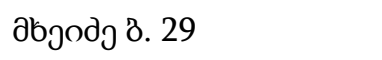 & 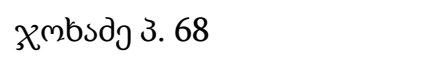 \\
\hline 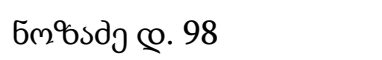 & Бежанишвили А. 78 \\
\hline byymsdg b. 54, 61 & Кирмелашвили Г. 78 \\
\hline
\end{tabular}




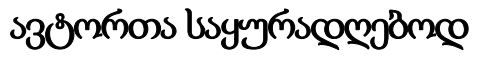

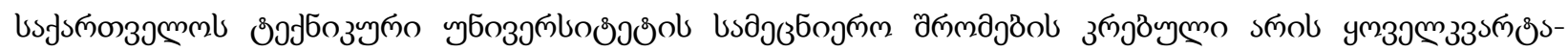

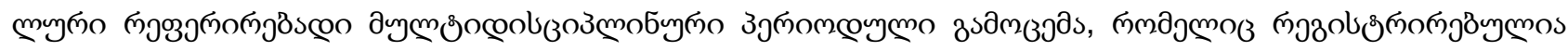

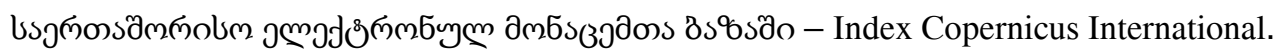

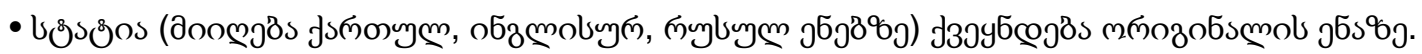

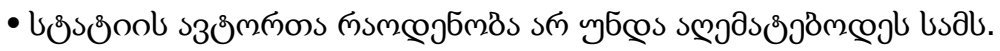

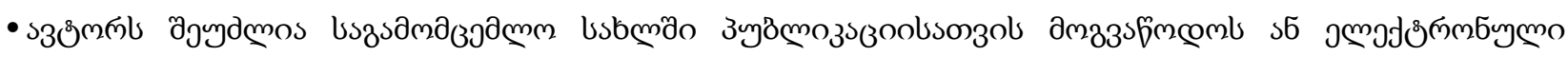

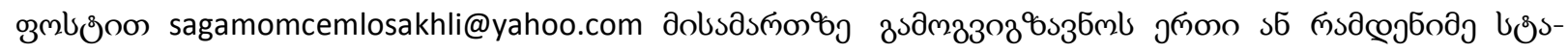

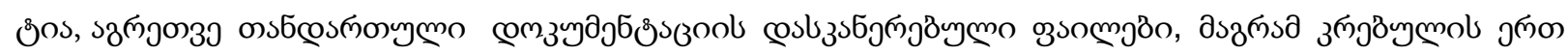

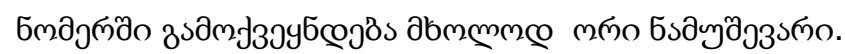

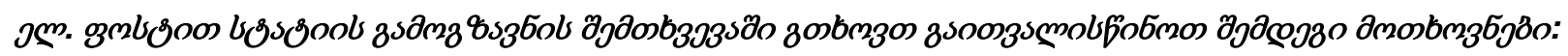

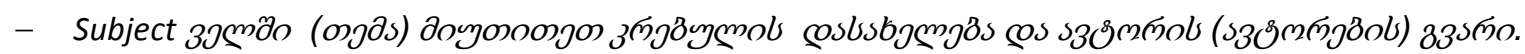

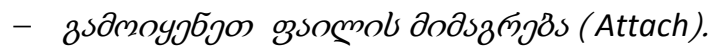

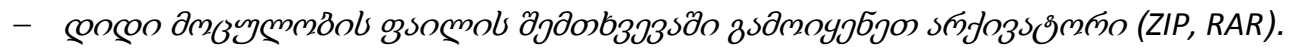

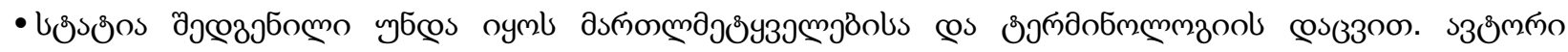

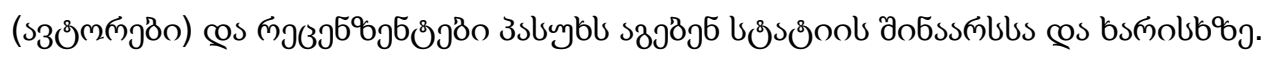

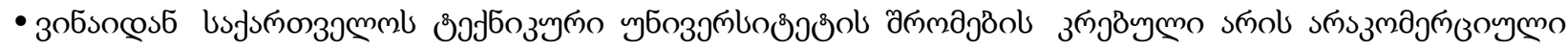

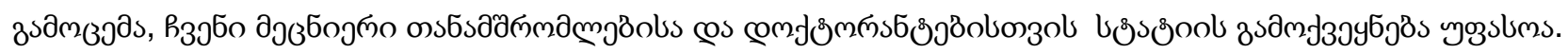

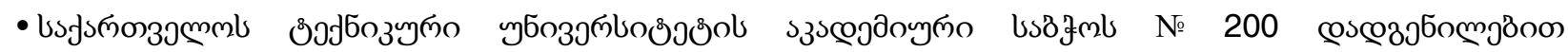

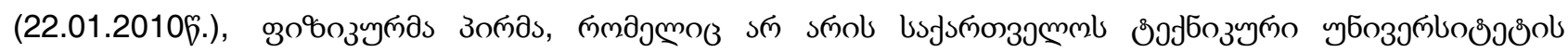

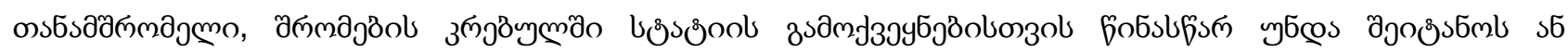

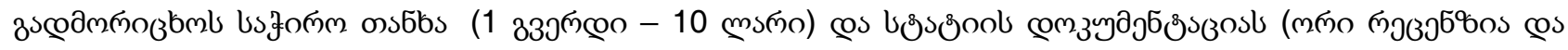

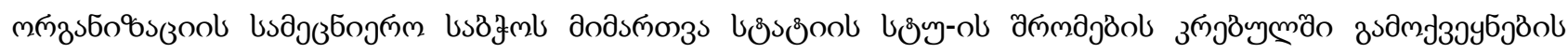

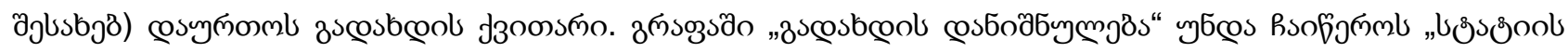

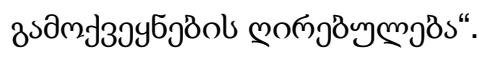

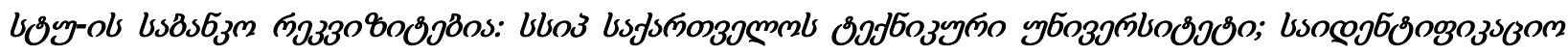

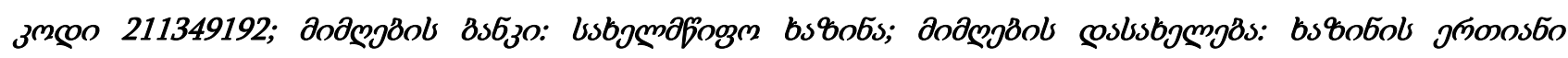

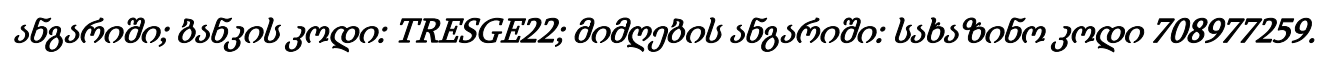

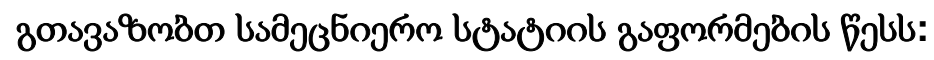

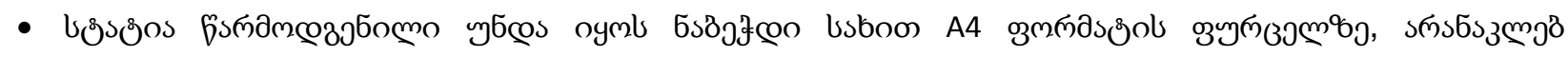

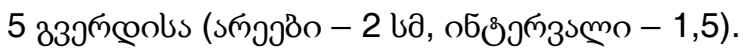

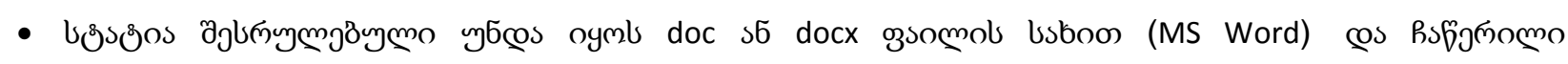

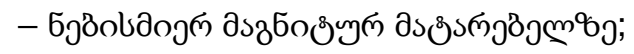

ISSN 1512-0996

www.shromebi.gtu.ge

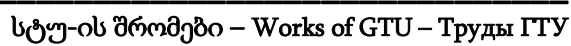

№3 (513), 2019 


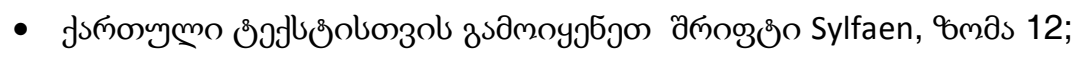

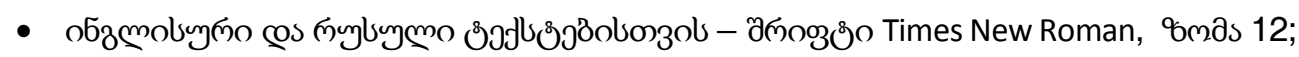

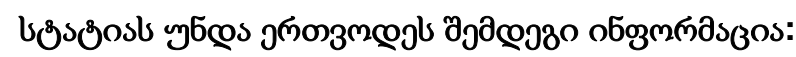

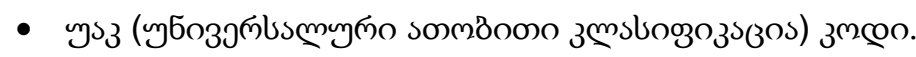

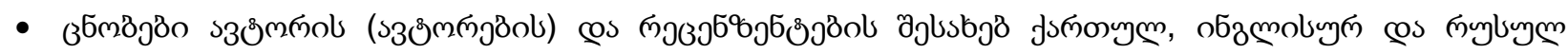
jбj3\%g:

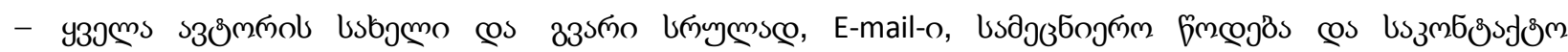

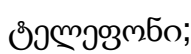

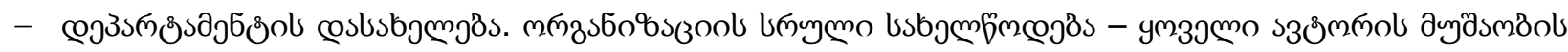

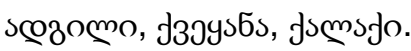

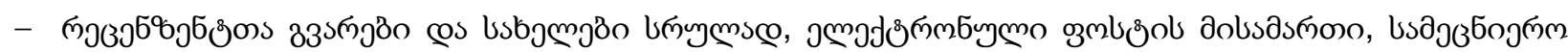

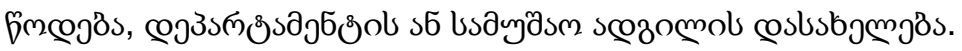

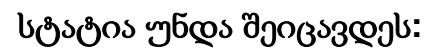

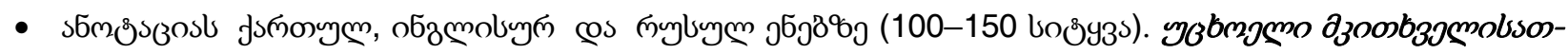

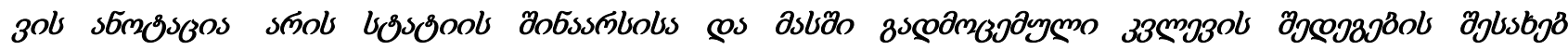

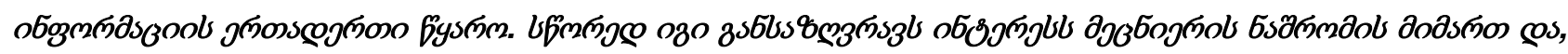

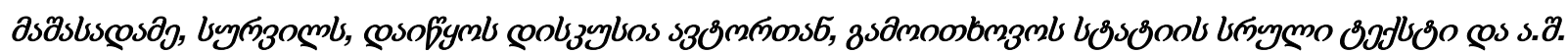

s6mossos y6cos oyml:

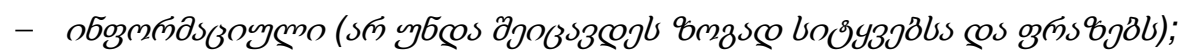

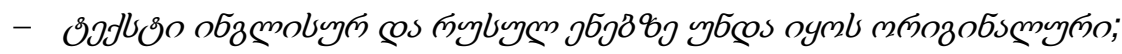

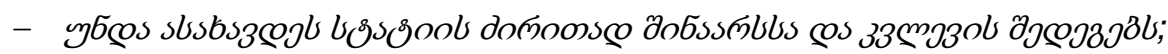

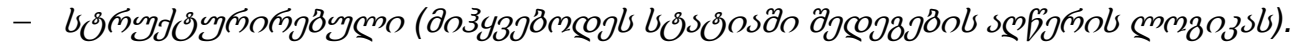

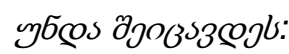

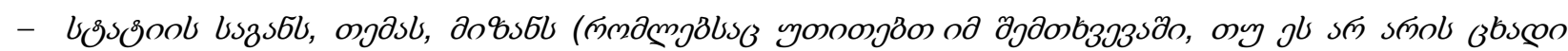
ussorool bsonsymogos6);

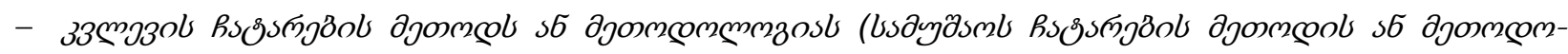

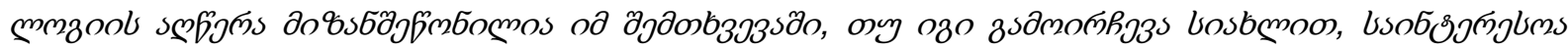

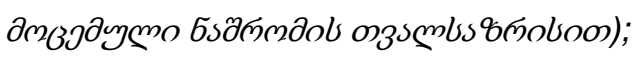

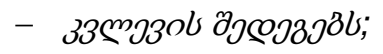

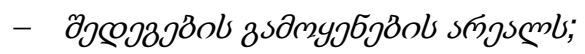

- costrzast;

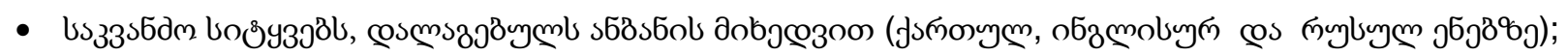

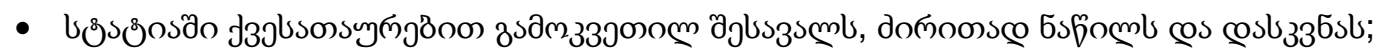




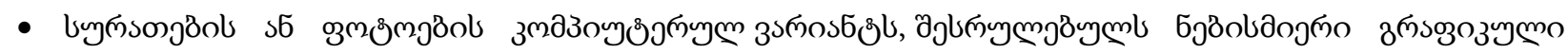

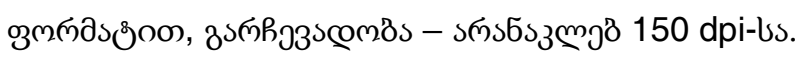

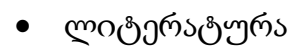

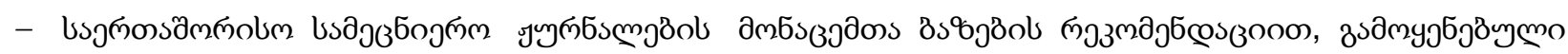

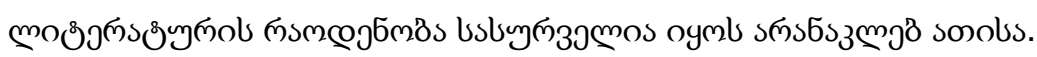

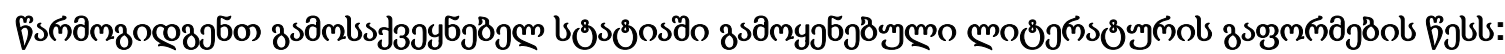

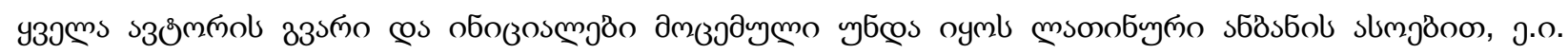

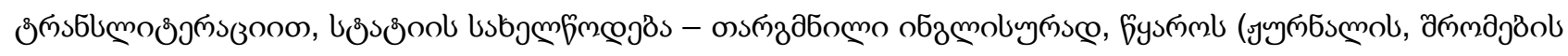

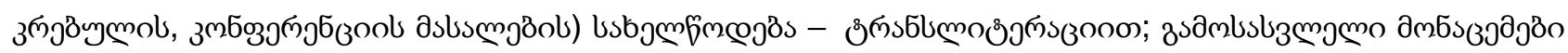

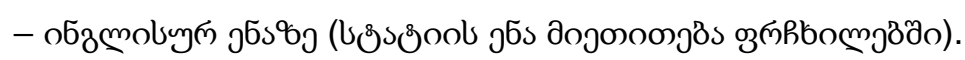

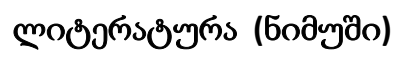

1. Jacques Sapir. Energy security as a common advantages. http://www.globalaffairs.ru/rumbler/n_7780 (In Russian).

2. "Official website of the International Energy Agency: http://www.iea org/topics/energysecurity/" (In English).

3. International Energy Agency “Key World Energy Statistics” 2014 (In English).

4. Energy strategy of France McDoleg_butenko20 May, 2009 (In Russian)

5. G.G. Svanidze, V.P. Gagua, E.V. Sukhishvili "Rene-wable energy resources of Georgia", Leningrad, Hydrometizdat, 1987, pp. 75-76 (In Russian).

6. Revaz Arveladze, Tengiz Kereselidze "The Georgian Full Independence of Electry Power Is Supported By Hydropower". Sakartvelos Teqnikuri Universitetis Archil Eliashvilis Saxelobis Martvis sistemebis Institutis Proceedings. N18 2014. Tbilisi (In Georgian).

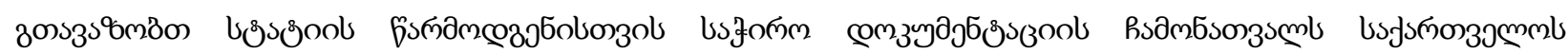

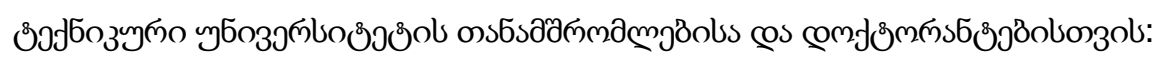

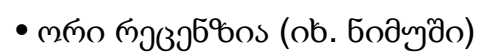

http://publishhouse.gtu.ge/site_files/recenziis nimushi.docx

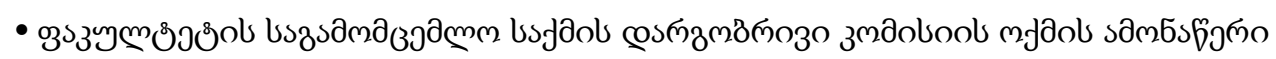

(ob. бodyəo) http://publishhouse.gtu.ge/site_files/aqtis forma.docx

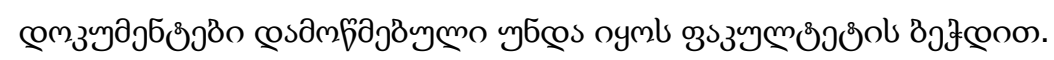

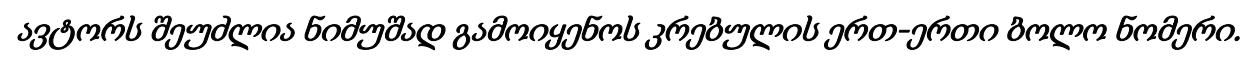




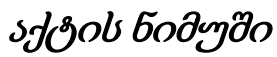

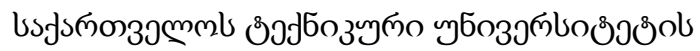

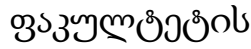

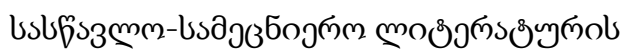

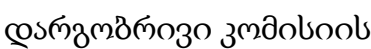

\section{ง ช ช ० №}

\section{ubcomasu jufр}

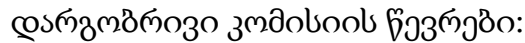

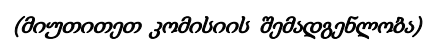

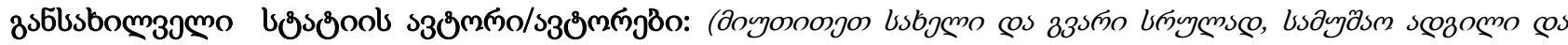

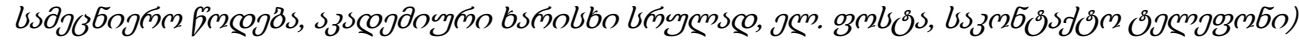

1.

2.

3.

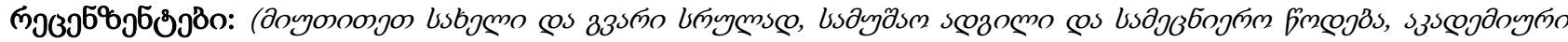

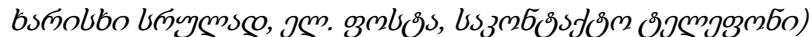

1.

2.

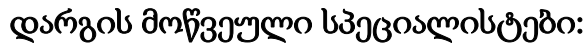

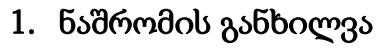

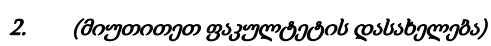

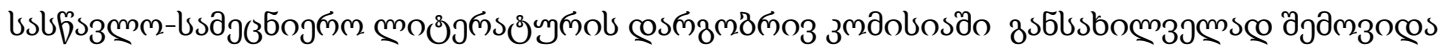

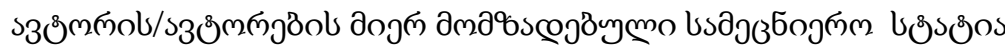

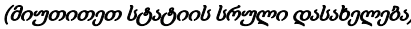

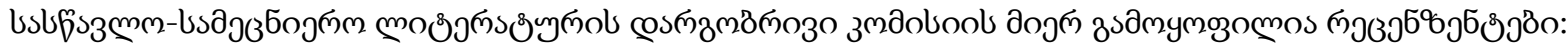
1 .

2. 


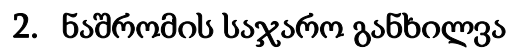

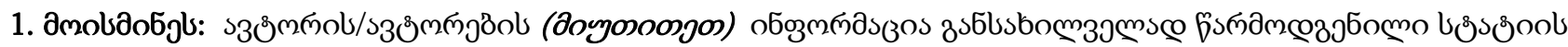
ajlubja.

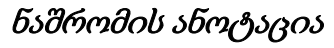

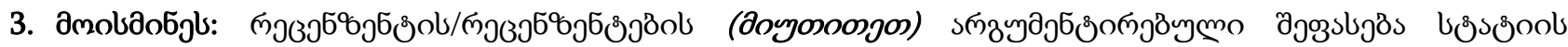

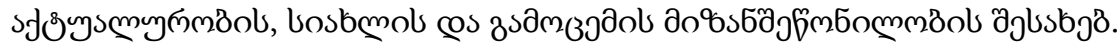

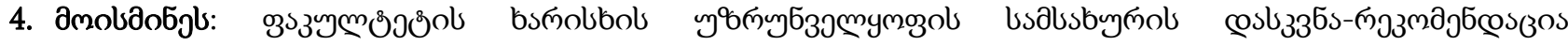

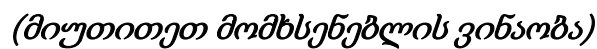

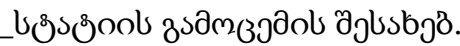

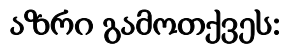

cosscozofjl:

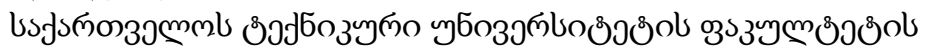

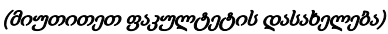

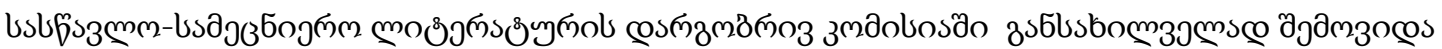

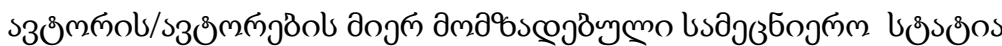

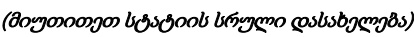

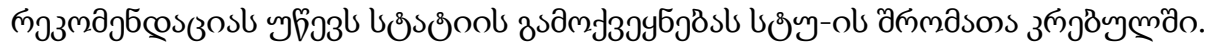

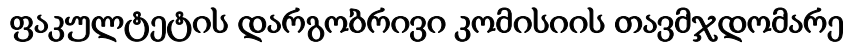

зmaobool acoozsбo

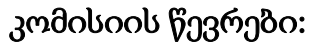

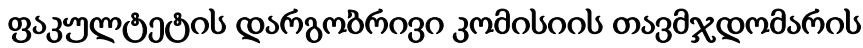

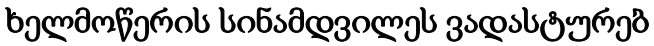

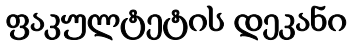

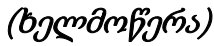




\section{๓эo3j68oob 6odyzo}

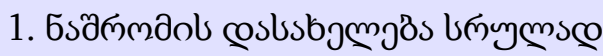

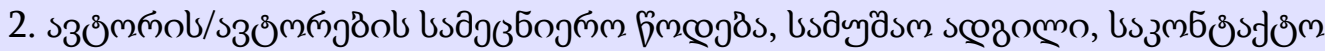

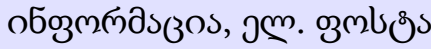

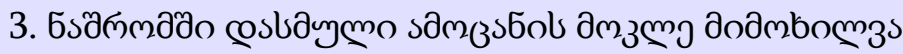

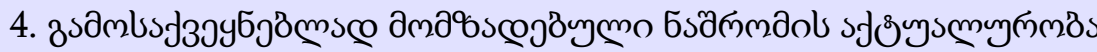

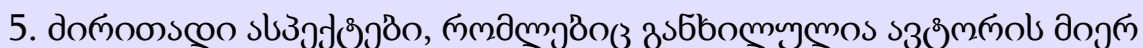

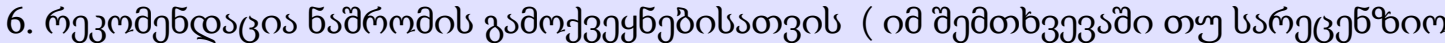

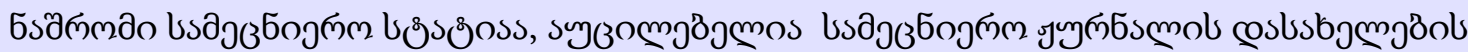
доono

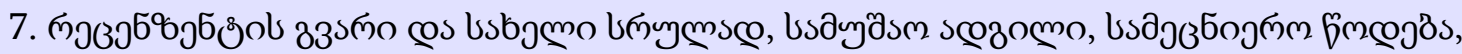

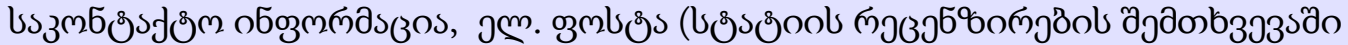
(пэ 


\section{Guidelines for Authors}

Collection of Academic Works of Georgian Technical University is a multidisciplinary quarterly refereed periodical included in Index Copernicus International.

- An article (accepted in Georgian, English or Russian) is published in the original language;

- The number of authors of an article should not exceed three;

- Authors should submit original copies of one or more articles for publication to the publishing house or send scan versions to sagamomcemlosakhli@yahoo.com along with supporting documentation, but only two articles from the same author(s) will be published in one edition;

\section{To submit scan versions via email please follow the instructions:}

- In the Subject line indicate the collection of works and the name(s) of author(s).

- Attach the file(s) properly;

- Use ZIP or RAR file compressors in case of large files to attach.

- The article should be literal, well-structured and apply proper terminology to convey the author's constructive arguments relevant to the subject. The authors and reviewers are responsible for the content and quality of an article;

- The collection of works of GTU is a non-commercial publication and running the articles of our researchers and for $\mathrm{PhD}$ students is free of charge;

- According to the Resolution No.200 of GTU Academic Council (22.01.2010), authors who are not the employees at the University, should make the preliminary payment by cash or transfer to have their paper published (10 GEL per page). Copy of the payment receipt should be enclosed with the supporting documentation (two reviews and a reference by the organization's academic board on publishing the article in GTU collection of scientific papers). "Cost of article publication" shall appear as subject in the "purpose of payment" field.

GTU bank details: LEPL Georgian Technical University; organization's identification number 211349192; beneficiary bank: State Treasury; beneficiary: joint treasury account; bank code: TRESGE22; Account number: treasury code 708977259.

How to form an academic article:

- The text should be presented in print-out form (A4), no less than 5 pages (margins - $2 \mathrm{~cm}$, line spacing - 1,5);

- Only MS Word versions of texts are accepted (doc or docx) presented electronically on any magnetic carrier;

- For Georgian texts: font - Sylfaen, font size - 12 pt;

- For English and Russian texts: font - Sylfaen, font size - 12 pt. 
The accompanying information to the article should include:

- Universal Decimal Classification (UDC)

- Information about the author(s) and reviewers in Georgian, English and Russian:

- Full name, academic title, email and phone number of each author;

- Department, full name of organization - place of employment of each author, area/town, country;

- Full name, email, academic title, department or place of employment of each reviewer.

The article should include:

- An abstract in Georgian, English and Russian (100-150 words long). For foreign readers an abstract is the only source of information about the content of an article and results of the research conveyed by it. An abstract therefore defines the reader's interest towards the article and possibility of further outreach to the author for the full text, etc.

An abstract should be:

- Informative (free of generalized terms and statements);

- Original (with quality translations in English and Russian with the proper application of terminology);

- Specific (conveying the core content of an article);

- Properly structured (consistent with the research results given in the article).

\section{An abstract should contain:}

- The subject, topic and objective of an article (indicated in case if these are not clear from the title);

- Method or methodology of research performed (expected to be described when and if this method or methodology are new and interesting with reference to the article);

- Research results;

- Area of application of research results;

- Conclusion.

- Key words sorted by alphabet (Georgian, English and Russian);

- Sections should be outlined Introduction, Main Part and Conclusion;

- Digital version of drawings or images in any graphic format, resolution $150 \mathrm{dpi}$;

- Reference

- By the recommendations of Databases of International Scientific Journals the number of references should be no less than ten.

How to form the reference section in the article:

Name and surname of each author should be given in Latin letter initials, title of the articles - translated in English, name of the source (journal, collection of works, conference materials) - with transliteration (original language of the article should be indicated in brackets). 


\section{References (sample)}

1. Jacques Sapir. Energy security as a common advantages. http://www.globalaffairs.ru/rumbler/n_7780 (In Russian).

2. "Official website of the International Energy Agency: http://www.iea org/topics/energysecurity/" (In English).

3. International Energy Agency "Key World Energy Statistics" 2014 (In English).

4. Energy strategy of France McDoleg_butenko20 May, 2009 (In Russian).

5. Svanidze G.G., Gagua V.P., Sukhishvili E.V. "Rene $\neg$ wable energy resources of Georgia", Leningrad, Hydrometizdat, 1987, pp. 75-76 (In Russian).

6. Revaz Arveladze, Tengiz Kereselidze "The Georgian Full Independence of Electry Power Is Supported By Hydropower". Sakartvelos Teqnikuri Universitetis Archil Eliashvilis Saxelobis Martvis sistemebis Institutis Proceedings. N18 2014. Tbilisi (In Georgian).

Requirements for the submission of articles by the employees and for $\mathrm{PhD}$ students of Georgian Technical University:

- Two reviews (see the sample at) http://publishhouse.gtu.ge/site_files/recenziis nimushi.docx

- Minutes of the sectoral committee of the faculty publishing (see the sample at) http://publishhouse.gtu.ge/site_files/aqtis forma.docx

Documents should be verified with the faculty stamp.

\section{Notice to Authors}

Authors may consider one of the previous editions of GTU Collection of Academic Works as an example 


\section{К сведению авторов}

Сборник научных трудов Грузинского технического университета является ежеквартальным мультидисциплинарным реферируемым периодическим изданием, которое зарегистрировано в международной базе электронных данных__ndex Copernicus International.

- Статьи (принимаются на грузинском, английском, русском языках) публикуются на языке оригинала.

- Количество авторов статьи не должно превышать 3.

- Автор может предоставлять для публикации в Издательском доме или по электронной почте (на следующий адрес: sagamomcemlosakhli@yahoo.com) одну или несколько статей, а также в сканированных файлах сопутствующую документацию, но в одном номере могут быть опубликованы только две работы.

- В случае статей, присылаемьх по эл. почте, просьба предусмотреть следующие требования:

- указать в эл. Subject-е название сборника (тема) и фамилию автора (авторов);

- использовать Attach (приложить файл);

- в случае большого объема файла применить архиватор (ZIP, RAR).

- Статья должна быть составлена грамотно, с соблюдением терминологии. Автор (авторы) и рецензенты несут ответственность за содержание и качество статьи.

- Поскольку сборник трудов Грузинского технического университета является некоммерческим изданием, для сотрудников статьи публикуются бесплатно.

- Согласно постановлению академического совета №200 (22.01.2010 г.), физическое лицо, не являющееся сотрудником университета, для публикации статьи в сборнике трудов должно заранее внести или перечислить необходимую сумму (1 страница стоит 10 лари) за статью и соответствующую документацию (две рецензии и направление научного совета организации о публикации статьи в сборнике трудов ГТУ), приложив справку об оплате. В графе «Назначение оплаты» следует записать «стоимость публикации статьи».

Банковские реквизиты ГТУ: Юридическое лицо публичного права (ЮЛПП); Грузинский технический университет; идентификационный код 211349192; банк приема; государственная казна; название получателя: единый счет казны; код банка: TRESGE22; счет получателя: код казны 708977259.

\section{Предлагаем порядок оформления научной статьи:}

- статья должна быть представлена в напечатанном виде на странице формата A4, содержать не меньше 5 страниц (поля - 2 см, интервал - 1,5); 
- статья должна быть выполнена в виде файла doc или docx (MS Word) и записана на любом магнитном носителе;

- для грузинского текста применять шрифт Sylfaen, размер 12;

- шрифт для английского и русского текстов Sylfaen, размер 12;

Статья должна сопровождаться следующей информацией:

- код УДК (Универсальная десятичная классификация).

Сведения об авторе (авторах) на грузинском, английском и русском языках:

- полностью имя и фамилия автора (авторов), E-mail, научная степень и контактный телефон;

- название департамента, полное название организации - место работы каждого автора - страна, город;

- полностью фамилии и имена рецензентов, адрес электронной почты, научное звание, название департамента или места работы.

\section{К статье должны прилагаться:}

- Аннотация на грузинском, английском и русском языках (100-150 слов). Для иностранньгх читателей аннотация является единственным источником информации о результатах исследований, приведенныхх в содержании статьи. Именно это опредедяет интерес ученого к работе и, соответственно, желание начать дискуссию с автором, познакомиться с полным текстом статьи и т.д.

\section{Аннотация должна быть:}

- информационной (не должна содержать общих слов и фраз);

- оригинальной (перевод на английском и грузинском языках должен быть качественный, при переводе следует использовать специальную терминологию);

- содержательной (должна отражать основное содержание статьи и результаты исследования);

- структурированной (следовать в статье логике описания результатов).

\section{Должна содержать:}

- предмет статьи, тему, цель (которые указывают в том случае, если это не ясно из заглавия статьи);

- метод или методологию проведенного исследования (описание метода или методологии проведенной работы целесообразно в том случае, если они выделяются новизной, интересны с точки зрения данной работы);

- результаты исследования;

- ареал использования результатов;

- выводы;

- ключевые слова, расположенные по алфавиту (на грузинском, английском и русском языках);

- в статье должны быть выделены подзаголовки: введение, основная часть и заключение (выводы);

- компьютерные варианты чертежей или фотографий должны быть выполнены в любом графическом

- формате, разрешением - не менее $150 \mathrm{dpi}$.

ISSN 1512-0996

www.shromebi.gtu.ge 
- Литература

По рекомендации базы данных международных научных журналов, число использованной литературы желательно должно быть не меньше 10.

Представляем порядок оформления в публикуемой статье использованной литературы:

Фамилия и инициалы всех авторов должны быть выполнены буквами латинского алфавита, т.е. транслитерацией; название статьи с переводом на английский язык; название источников (журнала, сборника трудов, материалов конференции) - транслитерацией (язык статьи указан в скобках).

\section{Литература (Образец)}

1. Jacques Sapir. Energy security as a common advantages. http://www.globalaffairs.ru/rumbler/n_7780 (In Russian).

2. "Official website of the International Energy Agency: http://www.iea org/topics/energysecurity/" (In English).

3. International Energy Agency "Key World Energy Statistics" 2014 (In English).

4. Energy strategy of France McDoleg_butenko20 May, 2009 (In Russian)

5. G.G. Svanidze, V.P. Gagua, E.V. Sukhishvili "Rene $\neg$ wable energy resources of Georgia”, Leningrad, Hydrometizdat, 1987, pp. 75-76 (In Russian).

6. Revaz Arveladze, Tengiz Kereselidze "The Georgian Full Independence of Electry Power Is Supported By Hydropower". Sakartvelos Teqnikuri Universitetis Archil Eliashvilis Saxelobis Martvis sistemebis Institutis Proceedings. N18 2014. Tbilisi (In Georgian).

Для представления статьи должен быть приложен перечень необходимых документов для сотрудников и докторантов Грузинского технического университета:

- две рецензии (см. образец)

http://publishhouse.gtu.ge/site_files/recenziis nimushi.docx

- выписка из протокола отраслевой комиссии по издательскому делу факультета (см. образец) http://publishhouse.gtu.ge/site_files/aqtis forma.docx документы должны быть удостоверены печатью факультета.

Автор может использовать в качестве образца один из последних номеров издания. 
https://doi.org/10.36073/1512-0996-2019-3

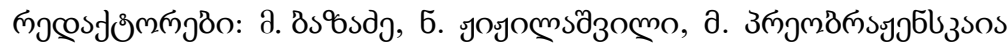

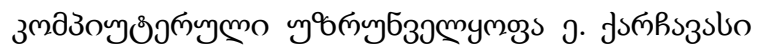

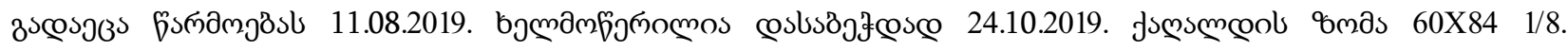

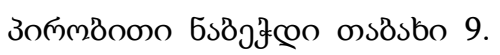

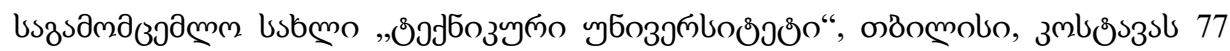

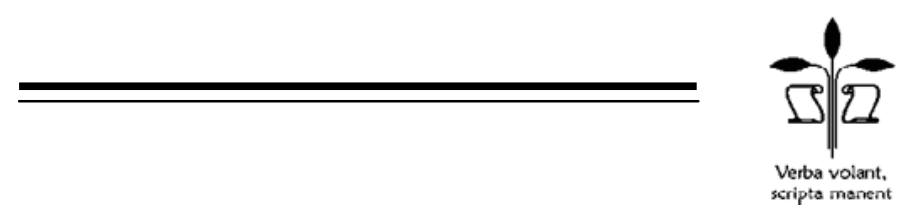

